\title{
Konzeption einer subjektunabhängigen, analytischen Unternehmensethik
}

„Business ethics is essential for business. Business ethics is vital not because it is fashionable - though business can ill afford to ignore anything [...] that seriously influences the markets in which it operates. Rather, business ethics is necessary because ethical choices are unavoidable. The business ethics challenge is to make that inevitable ethical decision-making explicit so as to make it better."1

Wie bereits im zweiten Kapitel dargelegt, befasst sich die Arbeit im Folgenden spezifisch mit dem Forschungsfeld der Unternehmensethik, sie fokussiert sich folglich auf Unternehmen als wertschöpfungsfokussierte Betriebe. Die Beschäftigung mit Unternehmen als originärem Erfahrungsobjekt der Unternehmensethik erscheint hierbei nicht nur einer konventionellen Bezeichnung des Forschungsfeldes nach, sondern auch inhaltlich gerechtfertigt. So sind zum einen Unternehmen für den Lebensstandard moderner Gesellschaften unverzichtbar. Sie sind nach WIElAND „das Rückgrat moderner Gesellschaften.“2 Ebenso stellt PFrIEM fest: „In marktwirtschaftlich-kapitalistisch verfassten Gesellschaften sind Unternehmensorganisationen $\mathrm{zu}$ besonders wichtigen Akteuren der Daseinsbewältigung geworden."3 Zum anderen befinden sich gerade Unternehmen - und hier insbesondere die meist von Aktiengesellschaften betriebenen Großunternehmen ${ }^{4}-$ im kritischen Fokus der Öffentlichkeit, da diese häufig über umfassende Ressourcen verfügen und im Kontext der Globalisierung zunehmend Handlungsmöglichkeiten

\footnotetext{
${ }^{1}$ Sternberg, E. (2018), S. 15.

${ }^{2}$ Wieland, J. (2005b), S. 15.

${ }^{3}$ Pfriem, R. (2007a), S. 81.

${ }^{4}$ Vgl. hierzu auch die konzeptionellen Überlegungen in Abschnitt 4.3.5.
} 
gewonnen haben. Sie sind damit besonders gefordert, die getroffenen Entscheidungen im Lichte der kritischen Öffentlichkeit reflektiert und überzeugend zu begründen. ${ }^{5}$

Eine grundlegende Bedeutung nimmt in diesem Zusammenhang der Begriff der Verantwortung ein, welcher ein zentrales konzeptionelles Fundament der weiteren Überlegungen darstellt und zum Ausgangspunkt der Begründung einer neuen Unternehmensethikkonzeption genommen wird. Wie im Weiteren gezeigt wird, ist dabei die Übernahme von Verantwortung nicht als reiner Moralappell zu begreifen, sondern gerade im Sinne einer notwendigen Sicherung der dauerhaften Existenz des Unternehmens zu begründen. Folglich besitzt die begriffliche Auseinandersetzung nicht nur akademische Bedeutung, sondern weist ebenfalls für die unternehmerische Praxis zunehmende Relevanz auf. So betont auch HEBESTREIT, dass Verantwortung einen Begriff repräsentiere, „der in der Wirtschaft Bedeutung bekommen hat. [...] Es sind auch die Unternehmen selbst, welche zunehmend [...] darum bemüht sind, sich als verantwortungsvolle Wirtschaftsakteure zu präsentieren."6 Auch ULRICH und KAISER stellen in diesem Sinne fest: „In einer sensitiveren Öffentlichkeit gewinnt der Umgang mit Fairnessund Verantwortungsfragen im Wirtschaftsleben an Bedeutung."

In einem ersten Schritt ist hierbei der Begriff der Verantwortung selbst einer konzeptionell-historischen Rezeption zu unterziehen, bevor der Träger der Verantwortung - klassisch: das Subjekt der Verantwortung - einer detaillierteren kritischen Analyse unterzogen wird. ${ }^{8}$ Hierauf aufbauend entwickelt die Arbeit die konzeptionellen Grundlagen einer systemtheoretisch fundierten Mesoethik, wobei auf die Erkenntnisse der neueren Systemtheorie zurückgegriffen wird. Darüber hinaus wird mit dem Analytischen Framework eine Möglichkeit der praktischen Umsetzung der unternehmensethischen Überlegungen erarbeitet. Abschließend werden auch die Konsequenzen zentraler Forschungsergebnisse für das Controlling als institutionalisierter Führungsunterstützung präsentiert und einige Überlegungen zur Relevanz einer kulturellen Verankerung ethischer Maßnahmen vorgestellt.

\footnotetext{
${ }^{5}$ So betont auch KLINE: ,There is truth to the claim that scholarly focus on large publicly traded corporations is justified due to their potential influence relative to that of a small firm." (Kline, W. (2018), S. 224).

${ }^{6}$ Hebestreit, N. (2015), S. 9.

${ }^{7}$ Ulrich, P. / Kaiser, M. (2001), S. 25.

${ }^{8}$ Die weiteren Ausführungen finden sich grundlegend auch in Lingnau, V. / Fuchs, F. (2019).
} 


\subsection{Verantwortung als Schlüsselbegriff einer angewandten Ethik und das Subjekt als relationaler Träger der Verantwortung}

\subsubsection{Zum Begriff der Verantwortung}

Eine Betrachtung der gegenwärtigen Literatur zur angewandten Ethik und insbesondere auch der Wirtschaftsethik als Teildisziplin zeigt die intensive Auseinandersetzung hinsichtlich der Zuschreibung und Begründung von Verantwortung der im Wirtschaftskontext tätigen Akteure. Der Begriff der Verantwortung kann dabei als eines der zentralen Paradigmen einer modernen, durch die Aufklärung geprägten Ethik aufgefasst werden ${ }^{9}$ und repräsentiert gerade auch für die angewandte Ethik eine Basisbegrifflichkeit. ${ }^{10}$ In diesem Sinne hebt auch BOHRMANN hervor, dass Verantwortung einen „Schlüsselbegriff“11 für die angewandte Ethik darstelle. Gleichsam unterstreicht auch SCHWEIDLER die Bedeutung dieses Konstrukts für die angewandte Ethik, welches er als ,reflektierte praktische Rationalität in sozialen Verantwortungsstrukturen"12 auffasst. Der Begriff der Verantwortung kann dabei in verschiedenen Begriffsverständnissen gebraucht werden. So unterscheiden z. B. LENK und MARING ${ }^{13}$ neben einer Handlungsverantwortung auch eine Rollen- bzw. Aufgabenverantwortung und nehmen eine Differenzierung in eine moralische, rechtliche sowie reflexive Verantwortung ${ }^{14}$ vor, während VAN DE POEL ${ }^{15}$ detailliert zwischen normativen wie deskriptiven Interpretationen des

\footnotetext{
${ }^{9}$ Vgl. Noll, B. (2013), S. 50.

${ }^{10}$ Vgl. Neuhäuser, C. (2011b), S. 120. Vgl. ähnlich auch Göbel, E. (2017), S. 33.

${ }^{11}$ Bohrmann, T. (2018), S. 25. Vgl. ähnlich auch Bayertz, K. (1995), S. 3-4.

${ }^{12}$ Schweidler, W. (2018), S. 15, in der Quelle ebenfalls herv.

${ }^{13}$ Vgl. Lenk, H. / Maring, M. (1993), S. 233-234.

${ }^{14}$ Diese Interpretation fokussiert auf eine Metaebene der „Begründung, Einführung oder Setzung“ (Lenk, H. / Maring, M. (1993), S. 235) der in der Verantwortungsrelation gültigen Normen.

${ }^{15}$ Vgl. Poel, I. v. d. (2015a), S. 14.
} 
Begriffs unterscheidet. Andere Autoren wie HEBESTREIT oder WERnER ${ }^{16}$ diskutieren wiederum vor allem den zeitlichen Aspekt, welcher zum einen ex ante bzw. prospektiv als prinzipielle Verantwortlichkeitsbeziehung aufgefasst werden könne, während ,retrospektive Verantwortung“ eine Ex-post-Zuschreibung von Verantwortung (i. d. R. nach einem Ereignis mit schwerwiegenden Folgen ${ }^{17}$ ) darstelle. $^{18}$

Beginnt man mit einer Analyse der Bezeichnung der ,Verantwortung“ selbst, so zeigt sich, dass diese bereits die Pflicht des „Antwortens“19 bzw. des „Rede-und-Antwort-Stehens“20 vor einer Instanz impliziert, wobei sich diese begrifflichen Überlegungen auch in anderen Sprachen wie dem Englischen

\footnotetext{
${ }^{16}$ Vgl. Hebestreit, N. (2015), S. 29-34; Werner, M. H. (2011a), S. 542; Brieskorn, N. (2010), S. 535. Vgl. auch Dembinski, P. H. (2017), S. 19-20; Ranson, I. (2010), S. 194-195. Dabei gilt jedoch, wie auch WERNER selbst hervorhebt, dass eine ex post festgestellte Verantwortung nur sinnvoll begründbar ist, sofern bereits prospektiv eine zuvor bestandene Verantwortungsrelation angenommen werden kann. Die folgenden Ausführungen fokussieren damit auf die konzeptionelle Durchdringung einer moralisch-prospektiven Verantwortungsrelation, welche dann aber wiederum retrospektiv Konsequenzen, etwa für die Legitimität eines Unternehmens, entfalten kann.
}

${ }^{17}$ Vgl. hierzu Bayertz, K. (1995), S. 5 und S. 14, welcher auch auf einige klassische Überlegungen bei RYLE hinweist: ,It makes sense, in this use, to ask whether a boy was responsible for breaking a window, but not whether he was responsible for finishing his homework in good time.“ (Ryle, G. (2000), S. 69). Eine Restriktion der Verantwortungsrelation auf die Folgen von Handlungen scheint jedoch konzeptionell noch zu eng. So werden Unternehmen (bzw. spezifisch das soziale System im Unternehmen) nicht nur für die Folgen (Teleologie) des Handelns verantwortlich gemacht. Sie sind vielmehr auch gefordert, dafür zu sorgen, dass prinzipiell richtig (d. h. gesellschaftlich legitim) gehandelt wird, da Unternehmen nicht nur an potentiellen Schäden ihrer Handlungen, sondern auch an den zugrunde liegenden Handlungen an sich und sogar Zielbildungsprozessen bzw. korporativen Intentionen (vgl. hierzu Neuhäuser, C. (2011a), S. 103-104) gesellschaftlich evaluiert werden.

${ }^{18}$ Vgl. auch die weiteren klassifikatorischen Überlegungen bei Seidel, P. (2011), S. 31-35; Röttgers, K. (2007), S. 19-20; Stahl, B. C. (2000), S. 226; Baier, K. (1998), S. 105-112; Kreikebaum, H. (1996a), S. 179-182; Lenk, H. (1993), S. 115-125; Kaufmann, F.-X. (1992), S. 13 und S. 40-46; Weischedel, W. (1972), S. 26.

${ }^{19}$ Vgl. hierzu auch Nida-Rümelin, J. (2011), S. 12; Schmidt, M. / Schank, C. / Vorbohle, K. (2011), S. 9; Filek, J. (2010), S. 411; Pfriem, R. (2008a), S. 458; Röttgers, K. (2007), S. 18; Maring, M. (2001a), S. 13; Stahl, B. C. (2000), S. 226; Thomé, M. M. (1998), S. 23; Kreikebaum, H. (1996a), S. 179; Lenk, H. (1993), S. 115; Weischedel, W. (1972), S. 15; Weischedel, W. (1946), S. 7.

${ }^{20}$ Sombetzki, J. (2014), S. 31; Werner, M. H. (2011a), S. 541. 
(,,responsibility“) oder Französischen (,responsabilité") widerspiegeln. ${ }^{21}$ Dabei verdeutlicht eine historische Analyse zur Begriffsentstehung, dass der Terminus der Verantwortung im deutschsprachigen Raum durchaus eine lange Begriffsgeschichte aufweist und sich bis in das 15 . Jahrhundert zurückverfolgen lässt. ${ }^{22}$ Hierbei handelt es sich jedoch noch vorherrschend um eine rechtliche Interpretation des Terminus. So entstammt die Bezeichnung dem mittelalterlichen Gerichtsvokabular, in welchem „Verantwortung“ relativ synonym zum Terminus der „Verteidigung“ bzw. Verteidigungsrede eines Beschuldigten gegenüber einer Rechtsinstanz (z. B. einem Gericht) verstanden wurde. ${ }^{23} \mathrm{Im}$ ethischen Kontext weist der Begriff dagegen noch eine relativ junge Begriffshistorie ${ }^{24}$ auf - ,zumindest als ethischer Schlüsselbegriff“ ${ }^{\star 25}$ - und wurde folgerichtig selbst zur Zeit NIETZSCHES noch meist als rechtlicher Fachterminus aufgefasst, wobei NIETZSCHE selbst mit dem von ihm genutzten Begriff der „Verantwortlichkeit“ ${ }^{\star 26}$ bereits einem modernen, moralisch interpretierten Begriffsverständnis folgte. ${ }^{27}$

Analog zur Entwicklung des Terminus in der deutschsprachigen Literatur lässt sich auch für die französisch- und englischsprachigen Veröffentlichungen der

${ }^{21}$ Vgl. Stahl, B. C. (2000), S. 226; Bayertz, K. (1995), S. 3. Dies gilt aber auch für viele außereuropäische Sprachen, wie z. B. NIDA-RUMELIN hervorhebt. Vgl. hierzu Nida-Rümelin, J. (2011), S. 12.

${ }^{22}$ Vgl. Bohrmann, T. (2018), S. 25; Dietzfelbinger, D. (2015), S. 48; Werner, M. H. (2011a), S. 543; Ranson, I. (2010), S. 194; Fetzer, J. (2004), S. 75; Grimm, J. / Grimm, W. (1956), S. 81.

${ }^{23}$ Vgl. Dietzfelbinger, D. (2015), S. 48; Sombetzki, J. (2014), S. 36; Vogelmann, F. (2013), S. 153-154; Hesseler, M. (2011), S. 154; Röttgers, K. (2007), S. 18; Fetzer, J. (2004), S. 75; Picht, G. (2004), S. 318-319; Thomé, M. M. (1998), S. 23; Ropohl, G. (1994), S. 110-111; Schwartländer, J. (1974), S. 1579.

${ }^{24}$ Vgl. Hebestreit, N. (2015), S. 17; Assadi, G. (2013), S. 59; Vogelmann, F. (2013), S. 149; Pfriem, R. (2008a), S. 458; Röttgers, K. (2007), S. 17; Stahl, B. C. (2000), S. 226; Staudigl, B. (2000), S. 146; Bayertz, K. (1995), S. 3. Der Gedanke, dass sich Menschen für ihr Handeln erklären bzw. rechtfertigen müssen, ist freilich älter und kann bereits in Schriften der Antike aufgefunden werden. So kann, wie auch SEIDEL hervorhebt, ,[v]on der späten Verwendung des Wortes Verantwortung [..] nicht darauf geschlossen werden, dass das Problem, das heute mit Verantwortung bezeichnet wird, nicht schon vorher existiert hätte oder diskutiert worden wäre." (Seidel, P. (2011), S. 27). Vgl. hierzu auch die Überlegungen in Nida-Rümelin, J. (2011), S. 11; Holl, J. (1980), S. 23-29; McKeon, R. (1957), S. 9.

${ }^{25}$ Fetzer, J. (2004), S. 75.

${ }^{26}$ So betont NIETZSCHE: „Eben das ist die lange Geschichte von der Herkunft der Verantwortlichkeit. Jene Aufgabe, ein Tier heranzuzüchten, das versprechen darf [...]." (Nietzsche, F. (1964), S. 28, in der Quelle gesperrt herv.).

27 Vgl. Vogelmann, F. (2013), S. 152-154. 
Ethik erst relativ spät, gegen Mitte bis Ende des 19. Jahrhunderts, eine entsprechende Begriffsverwendung nachweisen, so z. B. bei LUCIEN LÉVY-BRUHLS „L'idée de responsabilité “28 aus dem Jahre 1884 oder MiLLs Essay „On Liberty“29 von 1859. ${ }^{30}$ In diesem Sinne konstatiert BAYERTZ: „Erst in der zweiten Hälfte des 19. Jahrhunderts findet der Begriff größere Verbreitung, um im 20. Jahrhundert in den Rang einer ethischen Schlüsselkategorie aufzusteigen. “31 Damit zeigt sich, obschon der Begriff bereits am Rande z. B. auch bei HUME verwendet wird, doch lange Zeit ,eher die Marginalität des Begriffs; gebraucht wird überdies nicht das jeweilige Substantiv, sondern meist die Adjektive ,responsible', ,responsable " und ,verantwortlich'. Erst in der zweiten Hälfte des 19. Jahrhunderts wächst die Häufigkeit der Verwendung und tritt der substantivische Gebrauch in den Vordergrund. “32

Verantwortung als Leitgedanke einer Ethikkonzeption hat dabei spätestens im Laufe des 20. Jahrhunderts eine stark zunehmende Verbreitung im gesellschaftlichen wie auch spezifisch im wissenschaftlichen Diskurs gefunden. ${ }^{33}$ In diesem Sinne betont auch PRECHTL, dass dem Terminus der Verantwortung ,zunehmend der Status eines ethischen Prinzips zugesprochen" 34 wird. Auch SPAEMANN betont, dass in der Gegenwart „[d]as Wort Verantwortung [.] Konjunktur“35 habe. Aus wissenschaftlicher Perspektive führte MAX WEBER im politischen Kontext die bis heute häufig rezipierte Unterscheidung zwischen einer Gesinnungsethik - welche, wie aufgezeigt, teilweise kritisch (allerdings konzeptionell

\footnotetext{
${ }^{28}$ Vgl. Lévy-Bruhl, L. (1884), S. 1-10.

${ }^{29}$ Vgl. z. B. Mill, J. S. (1867), S. 64. In seinem Werk findet sich jedoch gerade zu Beginn noch ein stark legalistisches Verständnis (vgl. hierzu z. B. die Ausführungen auf S. 7).

${ }^{30}$ Vgl. Bayertz, K. (1995), S. 3.

${ }^{31}$ Bayertz, K. (1995), S. 3. Diese lange Zeit geringe Verbreitung im philosophischen Diskurs legt auch die vielfach fehlende Aufnahme in klassischen Lexika der Philosophie noch zu Beginn des 20. Jahrhunderts nahe. Vgl. hierzu Röttgers, K. (2007), S. 17. Ebenso betont auch VoGELMANN, dass in den einschlägigen „Wörterbüchern und Lexika [.] Verantwortung erst ab 1974 (!) - mit Johannes Schwartländers Artikel im Handbuch philosophischer Grundbegriffe - einer eigenständigen Reflexion für würdig erachtet [wurde]; davor wird Verantwortung in den philosophischen Nachschlagewerken allenfalls als Verweis auf Zurechnung geführt.“ (Vogelmann, F. (2013), S. 154-155, in der Quelle ebenfalls herv.).

32 Bayertz, K. (1995), S. 3.

${ }^{33}$ Vgl. Werner, M. H. (2011a), S. 543; Bayertz, K. (1995), S. 4. SchWARTLĀNDER führt dies auch auf die Erfahrungen des Ersten Weltkrieges zurück. Vgl. hierzu Schwartländer, J. (1974), S. 1580.

${ }^{34}$ Prechtl, P. (2008), S. 645.

35 Spaemann, R. (2007), S. 37, in der Quelle ebenfalls herv.
} 
zu sehr verkürzt) auf die Deontologie KANTS bezogen wird - und einer Verantwortungsethik ein, wobei WEBER sich besonders kritisch mit religiös fundierten Gesinnungsethiken auseinandersetzte. ${ }^{36}$ Als einer der bekanntesten zeitgenössischen Verantwortungsethiker kann dabei HANS JONAS erachtet werden, dessen Hauptwerk „Das Prinzip Verantwortung“ nicht nur akademisch, sondern auch im politischen Diskurs eine intensive Rezeption erfahren hat. ${ }^{37}$ In diesem befasst sich JONAS mit den durch die modernen Technologien induzierten gesellschaftlichen Risiken und hebt in diesem Kontext als Zentralnorm ${ }^{38}$ die Übernahme von Verantwortung - anstelle von alleinig gutem Willen oder dem Prinzip der Hoffnung - hervor. ${ }^{39}$ Das „Prinzip Verantwortung“ wird jedoch nicht nur im klassischen philosophischen Diskurs intensiv rezipiert, sondern hat in jüngster Zeit auch zunehmend in betriebswirtschaftlichen Publikationen Anwendung gefunden ${ }^{40}$, welches nochmals die Bedeutung dieses Begriffes unterstreicht.

\subsubsection{Konzeptionen der Verantwortungsrelation}

In der Ethikforschung, welche sich vertieft mit dem Terminus der Verantwortung befasst, weist insbesondere die konzeptionelle Durchdringung der Verantwortungsrelation eine zunehmende Bedeutung auf, wie das entstandene Schrifttum der vergangenen Jahre in diesem Forschungsfeld aufzeigt. Verantwortung kann

\footnotetext{
${ }^{36}$ Vgl. Weber, M. (1968), S. 57-65.

${ }^{37}$ Vgl. Noll, B. (2013), S. 54; Ranson, I. (2010), S. 203. So konstatiert in diesem Zusammenhang auch NIDA-RUMELIN: ,Der Begriff der, Verantwortung ' hatte eine ethisch und politisch motivierte Hochphase in den 1970er Jahren. Die politische Praxis erschien vielen Menschen zunehmend als unverantwortlich angesichts einer fortschreitenden Umweltzerstörung, angesichts der absehbaren Erschöpfung natürlicher Ressourcen, angesichts der Verelendung in der Dritten Welt.“ (Nida-Rümelin, J. (2011), S. 142).

${ }^{38}$ Diese lautet: „Handle so, dass die Wirkungen deiner Handlung verträglich sind mit der Permanenz echten menschlichen Lebens auf Erden.“ (Jonas, H. (1984), S. 36). Dass KANT kaum im primären Sinne als Gesinnungsethiker aufzufassen ist, wird nochmals dadurch deutlich, dass sich auch der verantwortungsethische Imperativ in JONAS Werk ganz klar an den kategorischen Imperativ KANTS anlehnt.

${ }^{39}$ Vgl. Jonas, H. (1984), S. 26-38. In der Literatur wird häufig darauf hingewiesen, dass der Titel als Abgrenzung zu BLOCHS Werk „Das Prinzip Hoffnung“ (vgl. einführend Bloch, E. (2016), S. 1-18) konzipiert wurde. Vgl. hierzu die Anmerkungen bei Viana, W. C. (2010), S. 90; Globokar, R. (2002), S. 435; Kornwachs, K. (2000), S. 57 sowie die Ausführungen bei Jonas, H. (1984), S. 376-387.

${ }^{40}$ So z. B. auch explizit bei Pappenheim, J. R. v. (2009), welcher das Prinzip der Verantwortung im Kontext eines nachhaltigen unternehmerischen Wirtschaftens gebraucht.
} 
in diesem Kontext nach FETZER als das „Eintreten(-Müssen) eines Subjekts für ein Objekt ${ }^{* 41}$ aufgefasst werden. Wie bereits diese Definition hervorhebt, bedarf es, als Minimalanforderung einer Verantwortungsrelation, eines Subjekts, welches Verantwortung trägt, sowie eines Objekts, auf welches sich die Verantwortung bezieht. Die genaue Ausgestaltung dieser Beziehung ist in der Literatur jedoch nicht unumstritten. So wurden seit der erstmaligen konzeptionellen Durchdringung des Verantwortungszusammenhangs von SCHUTz ${ }^{42}$ vielfältige Ansätze erarbeitet, welche sowohl im Umfang als auch in der genauen Bezeichnung der Elemente differieren.

Einfache, zweiteilige Modelle reichen dabei von der Betrachtung des Verantwortungsträgers gegenüber einer spezifischen Instanz bis hin zur reinen SubjektObjekt-Relation der Verantwortung. ${ }^{43}$ Verbindet man diese beiden Perspektiven, so erhält man eine klassische dreiteilige Konzeption der Verantwortungsrelation, in welcher ein Verantwortungssubjekt für ein Objekt (z. B. für ein Individuum) gegenüber einer Instanz Verantwortung trägt. ${ }^{44}$ Diese Perspektive fokussiert folglich auf die Frage: „Wer ist wofür wem verantwortlich?“45 Der aufgezeigte Ansatz kann nun wiederum um die Berücksichtigung der Normen, welche der Evaluation zugrunde liegen, erweitert werden, so dass man ein vierteiliges Relationalmodell der Verantwortung erhält, welches ein Subjekt, ein Objekt, die Instanz sowie die zugrunde gelegten Normen umfasst. ${ }^{46}$ Darüber hinaus hat die

\footnotetext{
${ }^{41}$ Fetzer, J. (2004), S. 88.

${ }^{42}$ Vgl. hierzu die Ausführungen bei Schütz, A. (1976).

${ }^{43}$ Vgl. z. B. Dembinski, P. H. (2017), S. 20; Poel, I. v. d. (2015a), S. 16; Baier, K. (1998), S. 105; Kaufmann, F.-X. (1992), S. 24; Schütz, A. (1976), S. 274.

${ }^{44}$ Vgl. Kornwachs, K. (2017), S. 162; Dietzfelbinger, D. (2015), S. 48-49; Nida-Rümelin, J. (2011), S. 23; Kiepas, A. (2010), S. 76; Seeger, S. A. (2010), S. 207; Zimmerli, W. C. / Aßländer, M. S. (2010), S. 43-44; Duff, R. A. (2007), S. 23; Picht, G. (2004), S. 319-320; Hirsch Hadorn, G. (2003), S. 145; Stahl, B. C. (2000), S. 226; Riedel, M. (1979), S. 221-222. Vgl. auch die Überlegungen bei Bayertz, K. (1995), S. 16, welcher in diesem Kontext jedoch eine dreiteilige Relation von Subjekt, Objekt und Bewertungsnorm diskutiert. Vgl. auch Göbel, E. (2020), S. 119-122, welche neben dem Subjekt, Objekt und Instanz der Verantwortung auch die Verantwortungsrelation selbst thematisiert.

45 Kleinfeld, A. (1998), S. 244.

46 Vgl. Bohrmann, T. (2018), S. 27; Loh, J. (2017), S. 51; Düwell, M. (2013), S. 183; Noll, B. (2013), S. 53; Buddeberg, E. (2011), S. 12; Werner, M. H. (2011a), S. 543; Brieskorn, N. (2010), S. 535; Lenk, H. / Maring, M. (1993), S. 224.
} 
Literatur auch einige umfangreichere Modelle erarbeitet, welche bis zu fünf ${ }^{47}$, sechs ${ }^{48}$ oder sogar sieben ${ }^{49}$ konstituierende Elemente enthalten können. ${ }^{50}$

Im Weiteren folgt die Arbeit dabei jedoch dem verbreiteten Ansatz, Verantwortung als vierstellige Relation aufzufassen, welche sich aus einem Verantwortungssubjekt als Träger der Verantwortung, einem Objekt, auf welches sich die Verantwortung bezieht, die zugrunde gelegten normativen Maßstäbe sowie einer Instanz (z. B. Gewissen oder religiöse, staatliche Autoritäten, Stakeholder etc.), gegenüber der die Antwortpflicht besteht, zusammensetzt. In diesem Sinne betont auch LOH, dass ,vier Relata - nämlich Subjekt, Objekt, Instanz und normative Kriterien - als tradierter Kern des Diskurses gelten können. “51 Diese Überlegungen fasst nochmals die nachfolgende Abbildung 4.1 zusammen.

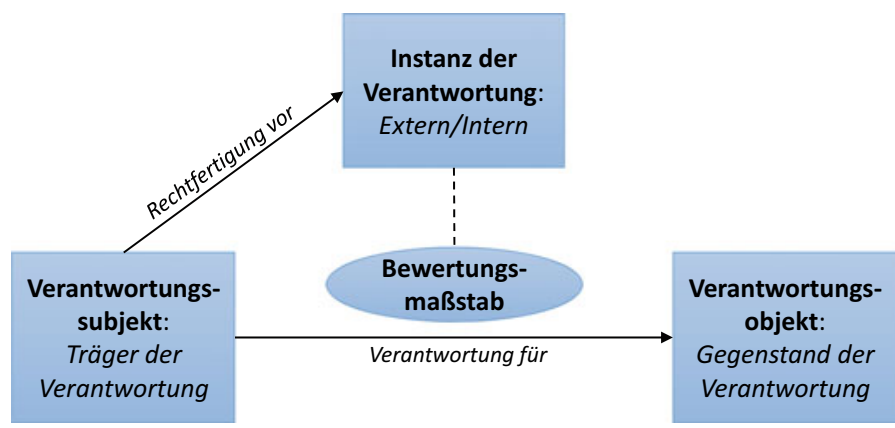

Abbildung 4.1 Das Relationalmodell der Verantwortung aus Verantwortungssubjekt, -objekt, Instanz und normativem Maßstab ${ }^{52}$

Das im vorigen aufgezeigte Subjektverständnis ist dabei allerdings zuerst einmal ausschließlich relational und benennt damit lediglich eine spezifische Position im Verantwortungszusammenhang. Damit ist jedoch über die Eigenschaften, also die Frage, weshalb ein solches Verantwortungssubjekt auch tatsächlich Verantwortung tragen kann und warum dieses Verantwortung tragen sollte,

${ }^{47}$ Vgl. z. B. Loh, J. (2017), S. 53; Sombetzki, J. (2014), S. 32; Seidel, P. (2011), S. 38-39; Kornwachs, K. (2000), S. 41.

48 Vgl. z. B. Maring, M. (2001a), S. 14; Lenk, H. / Maring, M. (1993), S. 229.

${ }^{49}$ Vgl. z. B. Ropohl, G. (1994), S. 111.

${ }^{50}$ Vgl. zur Übersicht auch Loh, J. (2017), S. 36; Werner, M. H. (2011a), S. 543.

${ }^{51}$ Loh, J. (2017), S. 51.

${ }^{52}$ In Anlehnung an Lingnau, V. / Fuchs, F. (2019), S. 234. 
noch nicht beantwortet. So weist auch die wissenschaftliche Literatur vielfach darauf hin, dass ein rein relationales Begriffsverständnis, wie dieses als grammatikalisches Subjekt in Aussagezusammenhängen regelmäßig auftritt, noch nicht hinreichend zur Attribution einer Verantwortungsrelation erachtet wird. Dies zeigt sich besonders klar in Aussagen, welche Gegenständen eine aktive Rolle zuweisen. In diesem Sinne fungiert in der Aussage „Der Ball rollt in das Tor“ der Ball zwar klar als grammatikalisches Subjekt, ohne jedoch evidenterweise philosophisch dem Gegenstand Verantwortung für die potentiellen Konsequenzen dieser Bewegung zuzuschreiben. Damit ist ersichtlich, dass ein rein grammatikalisches Subjektverständnis nicht als ausreichend erachtet werden kann, um den Status einer verantwortungsfähigen Einheit zu konstituieren, so dass, wie im Weiteren noch dargelegt wird, in der Literatur häufig auf einen bestimmten aufklärerisch-philosophischen Subjektbegriff rekurriert wird, um Verantwortung zu begründen. Diesen Gedanken betont auch FETZER wie folgt: „Der Windstoß ist für die zerbrochene Fensterscheibe nicht verantwortlich, selbst dann nicht, wenn er die einzige kausale Ursache war. In jeweils unterschiedlicher Bestimmung werden für Verantwortung auch Voraussetzungen auf Seiten des Subjekts postuliert. Dazu gehören z. B. die Intentionalität des Handelns, das Vorauswissen um die Folgen, die Kenntnis von Normen oder die Freiheit, auch anders entscheiden und handeln zu können." ${ }^{\text {53 }}$ Ähnlich stellt auch BUDDEBERG fest: ,[S]chlechte Witterungsbedingungen können zwar das Kentern des Schiffes kausal bewirkt haben, doch identifizieren wir sie deshalb in der Regel noch nicht als Subjekt einer Handlung. " ${ }^{4}$ In diesem Sinne betont letztlich auch LUBBE, dass noch „nicht jedes grammatische Subjekt [.] als solches schon ein Handlungssubjekt“"55 darstelle. Es muss somit gelten: „Das Subjekt muß [.] Subjekt der Verantwortung sein können." ${ }^{56} \mathrm{Um}$ dies zu ermöglichen, müssen dem Subjekt

${ }^{53}$ Fetzer, J. (2004), S. 91-92. Ähnlich subsumieren auch LENK und MARING: ,Zuschreibungs- und Zurechnungskriterium für Verantwortung ist die Fähigkeit zur Ausführung einer Handlung und nicht kausale Verursachung allein. Ein ursächlich wirksamer Windstoß ist nicht für die zerbrochene Fensterscheibe normativ verantwortlich zu machen.“ (Lenk, H. / Maring, M. (1993), S. 225). Zu den konzeptionellen Voraussetzungen einer Verantwortungszuschreibung vgl. auch die detaillierten Überlegungen bei Bayertz, K. (1995), S. 5-19.

${ }^{54}$ Buddeberg, E. (2011), S. 17. Vgl. zu ähnlichen Überlegungen auch Sombetzki, J. (2014), S. 37; Seidel, P. (2011), S. 43-44.

${ }^{55}$ Lübbe, W. (1998), S. 31. Vgl. hierzu auch Buddeberg, E. (2011), S. 18-19.

${ }^{56}$ Kornwachs, K. (2000), S. 48, in der Quelle fett herv. In diesem inhaltlich vorgeprägten Sinne konstatiert auch DIETZFELBINGER: „Verantwortung übernehmen kann immer nur ein Subjekt.“ (Dietzfelbinger, D. (2015), S. 48). 
folglich bestimmte Eigenschaften zugeschrieben werden, die z. B. im Selbstbewusstsein, der Willensfreiheit bzw. Autonomie einer Entität, welche intentionales Tun, d. h. Handeln, ermöglicht, der hinreichenden Kontrolle der Handlungsfolgen $^{57}$ oder dem Wissen über die Folgen aufgefunden werden können, ${ }^{58}$ welche das Subjekt zu einem moralfähigen Subjekt im philosophischen Sinne werden lassen. 59

Der Subjektbegriff selbst ist dabei überaus schillernd und unterlag im Laufe der Philosophiegeschichte nicht nur einem steten Wandel, sondern wurde gerade auch in den letzten Jahrzehnten inhaltlich zunehmend im philosophischen Kontext mittels verschiedener Argumentationslinien problematisiert. In diesem Zusammenhang wird sogar in Teilen des wissenschaftlichen Diskurses die Sinnhaftigkeit eines Subjektbegriffs ganz infrage gestellt oder gar ein ,,Verschwinden des Subjekts“60 postuliert. Allerdings stellt sich die Frage, welches Subjektverständnis dabei genau kritisiert wird - insbesondere da selbst die Subjektkritiker sich stets auf eine bestimmte Interpretation des Terminus fokussieren, welcher dann aus diversen Gründen verworfen wird. Eine konzeptionell saubere Formulierung des Subjektbegriffes, welcher in dieser Arbeit kritisch rezipiert wird, ist folglich notwendig, denn andernfalls droht „,das ,Verschwinden des Subjekts“ [.] zu einem Gemeinplatz der Diskussion zu werden, der lediglich die fehlende Begriffsbestimmung verdeckt, die uns befähigen würde $\mathrm{zu}$ beschreiben,

\footnotetext{
${ }^{57}$ In der modernen Literatur wird diese Voraussetzung auch häufig unter dem Begriff der Controllability diskutiert. Der zugrunde liegende Gedanke ist jedoch schon recht alt und findet sich so z. B. auch in der Eudemischen Ethik von ARISTOTELES: „,[B]ei allen Handlungen, deren Ursprung und Herr der Mensch ist, besteht offenkundig die Möglichkeit, daß sie geschehen oder daß sie nicht geschehen [...]. Alles Tun aber, dessen Vollzug oder Nichtvollzug von ihm abhängt, für dieses ist er persönlich der Urheber. Und überall wo er Urheber ist, da hängen die Dinge von ihm ab. [...] Und alles was er tut weil er sich dafür entschieden hat, das tut er selbstverständlich als willentlich Handelnder.“ (Aristoteles (1984), 1223a 4-19).

${ }^{58}$ Vgl. Stahl, B. C. (2000), S. 227.

${ }^{59}$ Vgl. konzeptionell auch Buddeberg, E. (2011), S. 18; Kornwachs, K. (2000), S. 48. Wie im Weiteren gezeigt wird, ist dabei im Kern der Verantwortungsfähigkeit auf subjektiver Seite das Selbstbewusstsein, die Erkenntnisfähigkeit sowie die Fähigkeit zu Handeln (potentielle Kausalität) notwendig, welche mit einem bestimmten aufklärerischen Subjektbegriff korrespondiert, sowie objektiv die Rahmenbedingungen in einer Situation, um als verantwortlich gelten zu können.

${ }^{60}$ Vgl. z. B. die Ausführungen bei Bürger, P. (2001); Mutschler, H.-D. (1994). Die Formulierung geht auf Überlegungen von FOUCAULT zurück, welcher auf das zunehmend unterstellte Verschwinden von ehemals als zentral erachteten menschlichen Fähigkeiten hinwies (vgl. Foucault, M. (1983), S. 462). FoUCAULT wird in diesem Zusammenhang in der Literatur oftmals auch die Formulierung des ,Tods des Subjekts“ zugeschrieben.
} 
was eigentlich verschwindet oder gar verschwunden ist“61 und in welcher „die Rede vom Verschwinden der Subjektivität oder des Subjekts in ein Metapherngestöber ausartet." ${ }^{62}$ In diesem Sinne hebt BAUMGARTNER kritisch hervor, dass ,[d]ie Abstraktheit dieser Rede vom Subjekt [.] ein [.] kritischer Gesichtspunkt “63 sei, denn ,wenn man sich die publizierten Vorträge oder Texte, in denen über das Subjekt gehandelt wird, gründlich ansieht, so erkennt man, daß nur die wenigsten differenziert $[\ldots]$ sind [...].“64 So sei, wie ähnlich auch Sснотт hervorhebt, häufig ,weitgehend unterbestimmt, was übergreifend eigentlich unter Subjekt $\mathrm{zu}$ verstehen ist." ${ }^{65}$ Eine solche Bestimmung ist jedoch erforderlich, ,da der Begriff des Subjekts zu oft als leere Hülse fungiert, der dann aufgrund seiner Unbestimmtheit alle möglichen Systemaufgaben übernehmen kann. “66 Um folglich nicht einer inhaltlich vagen Begriffsbestimmung zu unterliegen ${ }^{67}$, wird im Folgenden der Begriff des Subjekts begriffshistorisch in seiner Entwicklung in kompakten Zügen nachgezeichnet und einer detaillierten konzeptionellen Untersuchung unterzogen, um ein genaueres Verständnis des dieser Arbeit zugrunde liegenden philosophischen Subjektbegriffs zu erhalten, welcher in klassischen Ethikkonzeptionen zur Begründung von Verantwortungsfähigkeit herangezogen wird. ${ }^{68}$

${ }^{61}$ Zima, P. V. (2017), S. 2.

${ }^{62}$ Baumgartner, H. M. (1994), S. 19.

${ }^{63}$ Baumgartner, H. M. (1994), S. 25.

${ }^{64}$ Baumgartner, H. M. (1994), S. 25.

65 Schott, E. (1994), S. 122.

${ }^{66}$ Schott, E. (1994), S. 122.

${ }^{67}$ In diesem Sinne gilt wiederum nach STADLER: „Begriffsklarheit ist die Voraussetzung ernster Forschung; die Schaffung einwandfreier Begriffe und ihre Vereinigung zu einem geschlossenen System, erste und grundlegende Aufgabe jeder Wissenschaft." (Stadler, M. (1932), S. 5). Zudem kann auch konstatiert werden, dass ideengeschichtlich (so z. B. für eine aufgeklärte, humanistische Philosophie) der Begriff selbst von überragender Bedeutung ist, wie z. B. BEER und GRUNDMANN hervorheben: „Das Subjekt muss als Referenz der wissenschaftlichen Betrachtung ernst genommen werden. Als umkämpftes Terrain jedenfalls hat der Begriff des Subjektiven damit einen Siegeszug innerhalb der Ideengeschichte zu verzeichnen, den ansonsten kaum ein Terminus erreicht hat.“ (Beer, R. / Grundmann, M. (2004), S. 1).

${ }^{68}$ Dies betonen auch BEER und GRUNDMANN: „Politisches, moralisches und ethisches Handeln finden allesamt vor dem Hintergrund einer Subjektvorstellung statt, die in Form von Individualität als Quelle der Legitimation nicht mehr hintergangen werden kann.“ (Beer, R. / Grundmann, M. (2004), S. 1). 


\subsection{Zum philosophisch-aufklärerischen Subjektbegriff als Verantwortungsträger}

\subsubsection{Etymologie des Subjektbegriffs und Aufstieg zum philosophischen Zentralbegriff der Aufklärung}

Wie die vorigen Ausführungen bereits andeuteten, ist der Begriff des Subjekts sowohl im wissenschaftlichen Diskurs als auch im alltäglichen Sprachgebrauch überaus schillernd und weist eine beachtliche konzeptionelle Breite auf. ${ }^{69}$ Dabei wird auch deutlich, dass der Subjektbegriff in der Literatur recht heterogen verwendet wird. So herrscht zum einen bereits je nach zugrunde liegender Fachrichtung und Zeit der Veröffentlichung oft ein im Kern differierendes Begriffsverständnis $\operatorname{vor}^{70}$ - sofern die Begriffsproblematik überhaupt thematisiert wird. In diesem Sinne kann festgestellt werden, dass der Subjektbegriff von verschiedensten Disziplinen gebraucht wird und sein begrifflicher Inhalt, insbesondere im Rahmen philosophischer Überlegungen, den Gegenstand überaus kontroverser Auseinandersetzungen konstituiert. ${ }^{71}$ Folglich konstatiert auch ZIMA, dass dieser Begriff trotz aller Eingrenzungsversuche von einer ,schillernde[n] Vagheit" ${ }^{672}$ gekennzeichnet sei. HEIDEMANN spricht in diesem Zusammenhang sogar

${ }^{69}$ So konstatiert auch ZIMA: „Tatsächlich wohnt der Subjektbegriff nahezu allen theologischen, philosophischen, psychologischen, soziologischen und literaturwissenschaftlichen Ansätzen inne [...].“ (Zima, P. V. (2017), S. 31). Auch ScHULEIN betont: „Der Subjektbegriff ist ausgesprochen vielfältig und vieldeutig. [...] Die Heterogenität der Begriffe spiegelt die vielfältigen Bezüge des Themas. Subjekt(ivität) ist offensichtlich nicht ein konzeptionell isolierbarer, monologischer Gegenstand, sondern eine besondere Realität, die unterschiedliche Thematisierung zuläßt, ja erfordert - und sich einer definitiven Festlegung praktisch wie begrifflich entzieht.“ (Schülein, J. A. (2000), S. 657). In diesem Sinne hebt auch KIESELHORST hervor: „Der Begriff des Subjektes ist äußerst komplex und schwer zu fassen. Er kann in vielerlei Hinsicht definiert und betrachtet werden [...]." (Kieselhorst, M. (2010), S. 39). Vgl. für die weiteren Ausführungen grundlegend auch Lingnau, V. / Fuchs, F. (2019). ${ }^{70}$ Vgl. zum Überblick z. B. Zima, P. V. (2017), S. 1; Neuser, W. (2013), S. 29-30; Reckwitz, A. (2012b), S. 11-12; Beer, R. / Grundmann, M. (2004), S. 1-2; Tuschling, B. (2002), S. 5758; Schülein, J. A. (2000), S. 657; Baumgartner, H. M. (1994), S. 22-24. Darüber hinaus sei angemerkt, dass die weiteren Ausführungen sich primär mit der etymologischen Entwicklung im europäischen Diskurs beschäftigen, da dieser den aufklärerischen Subjektbegriff hervorgebracht und entscheidend geprägt hat.

${ }^{71}$ Vgl. Zima, P. V. (2017), S. 31.

72 Zima, P. V. (2017), S. 1. 
von „Begriffsverwirrungen“"73 bzw. vom „philosophischen Problembegriff ,Subjektivität‘،"74 Ähnlich subsumieren auch GELHARD, ALKEMEYER und RICKEN: „Das Problem der Subjektivität ist für die Philosophie seit Descartes immer eine Provokation geblieben. Besonders deutlich zeigt sich das in der Philosophie des 20. Jahrhunderts, wo der Anspruch, dieses Problem erstmals angemessen zu beantworten, und der Versuch, den Begriff des Subjekts endgültig loszuwerden, auf höchst unübersichtliche Weise ineinander gehen." ${ }^{\text {"75 }}$ Ziel der folgenden Ausführungen ist dabei nicht, vollumfassend jede Variation des Subjektbegriffs der Wissenschaftsgeschichte der Philosophie nachzuzeichnen, denn dies wäre fraglos, wie auch HEIDEMANN hervorhebt, ,ein eigenes philosophisches Großprojekt" ${ }^{\text {“76. }}$. Vielmehr seien im Folgenden die Kerneigenschaften des Subjektbegriffs identifiziert, welche, insbesondere in einer aufgeklärten Interpretation, zur inhaltlichen Begründung der oben aufgezeigten Verantwortungsrelation klassisch subjektphilosophisch herangezogen werden - aber auch zunehmend in die Kritik geraten sind.

Beginnt man sich dem Subjekt begriffsetymologisch zu nähern, so zeigt sich, dass trotz der teilweise stattgefundenen inhaltlichen Verschiebung im Laufe der Zeit dieser Begriff eine lange Verwendungsgeschichte aufweist. ${ }^{77}$ Die deutschsprachige Bezeichnung „Subjekt“ geht dabei auf den lateinischen Terminus des subiectum zurück, welcher wiederum eine Übertragung aus dem griechischen نлокєі́ $\mu \varepsilon v o v$ (hypokeímenon) darstellt. ${ }^{78}$ Dabei wird in der Literatur vielfach die potentielle Doppeldeutigkeit des Begriffs Subjekt hervorgehoben, welcher

\footnotetext{
${ }^{73}$ Heidemann, D. H. (2002), S. 11.

${ }^{74}$ Heidemann, D. H. (2002), S. 11. Teilweise wird in der Literatur, so z. B. von HAGENBUCHLE, noch eine differenziertere Betrachtung der Termini „Subjekt“ und „Subjektivität“" gefordert, wobei dieser z. B. konstatiert, dass Subjektivität noch ein relativ junger Begriff des 19. Jahrhunderts im Vergleich zum bereits seit Jahrhunderten gebrauchten Begriff des Subjekts sei. Vgl. hierzu Hagenbüchle, R. (1998), S. 4.

75 Gelhard, A. / Alkemeyer, T. / Ricken, N. (2013), S. 9.

${ }^{76}$ Heidemann, D. H. (2002), S. 12. Vgl. ähnlich auch Ornella, A. D. (2010), S. 92-93.

77 So finden sich erste Überlegungen bereits in der Antike, wenngleich noch ohne den heutigen expliziten Begriff zu besitzen. REICHERTz bezeichnet in diesem Kontext z. B. SOKRATES als ,wesentlichen Geburtshelfer des europäischen Subjekts.“ (Reichertz, J. (2010a), S. 37). Vgl. auch die dort referenzierte Literatur, insbesondere Foucault, M. (2004), S. 15-37.

${ }^{78}$ Vgl. Kratochvíl, Z. (2016), S. 27; Kirchner, F. et al. (2013), S. 637; Körtner, U. H. J. (2011), S. 150; Ornella, A. D. (2010), S. 81; Schöndorf, H. (2010c), S. 477; Seubert, H. (2005), S. 21; De Mul, J. (2004), S. 309; Schnädelbach, H. (2004), S. 60; Cichy, H. (2001), S. 24-28; Hagenbüchle, R. (1998), S. 4; Simon, J. (1998), S. 762. Die erstmalige Übersetzung wird in der Literatur teilweise CICERo (vgl. z. B. Kratochvíl, Z. (2016), S. 27 und S. 66) aber auch Boethius (vgl. z. B. Moro, A. (2017), S. 21) zugeschrieben. SEUBERT weist
} 
sowohl auf etwas Zugrundeliegendes bzw. Grundlegendes wie auch etwas Unterworfenes rekurrieren kann. So definiert etwa ZIMA: „Subjekt ist, etymologisch betrachtet, ein zweideutiges Wort, das sowohl Zugrundeliegendes (hypokeimenon, subiectum) als auch Unterworfenes (subiectus = untergeben) bedeutet, so daß in der Philosophie beide Aspekte zum Tragen kommen, bisweilen sogar im selben Diskurs, etwa bei Hegel.“79 Ähnlich subsumiert auch RECKWITZ: „Subiectum, das Subjekt hat eine doppelte Bedeutung: es ist das in die Höhe Erhobene und das Unterworfene. Es ist Zentrum autonomen Handelns und Denkens [...]. Und es ist das, was übergeordneten Strukturen unterliegt - bis hin zum Rechtssubjekt und zu jenem, für den im Englischen gilt: , he is subjected to something'. In seiner Doppeldeutigkeit präsentiert sich das Subjekt als ein unterworfener Unterwerfer, ein unterwerfendes Unterworfenes. "80 Auch einige klassische Autoren heben vielfach diese Zweideutigkeit hervor. So betont beispielsweise FOUCAULT: „Das Wort ,Subjekt‘ hat zwei Bedeutungen: Es bezeichnet das Subjekt, das der Herrschaft eines anderen unterworfen ist [...] und es bezeichnet das Subjekt, das durch Bewusstsein und Selbsterkenntnis an seine eigene Identität gebunden ist. “81 Die aufgezeigte Zweideutigkeit des Begriffs lässt sich wiederum mit dessen historischer Entwicklung in Verbindung bringen. Grundsätzlich kann dabei festgehalten werden, dass, obschon beide Aspekte eine gewisse Rolle spielen, bis in die Neuzeit lange Zeit die philosophische Begriffsauffassung als etwas Zugrundeliegendes existierte, wobei die Frage nach dem Bezug bzw. der genauen

allerdings darauf hin, dass beide Autoren ,subiectum“ noch relativ synonym zu „obiectum“ gebrauchen (vgl. Seubert, H. (2005), S. 21).

${ }^{79}$ Zima, P. V. (2017), S. 3, in der Quelle ebenfalls herv.

${ }^{80}$ Reckwitz, A. (2012a), S. 9. Vgl. ähnlich auch Reckwitz, A. (2012b), S. 14. Vgl. auch die Überlegungen bei KIESELHORST, welcher hervorhebt: „Denn der Begriff des Subjektes betont zum einen die Souveränität des Menschen in der Gestaltung seiner Umwelt, zum anderen aber auch die Abhängigkeit von gesellschaftlichen Verhältnissen. Des Weiteren enthält der Begriff den Verweis auf die Selbstbezüglichkeit und die Reflexion innerer Vorgänge.“ (Kieselhorst, M. (2010), S. 39). Vgl. auch die Überlegungen bei Füssel, M. (2003), S. 141.

${ }^{81}$ Foucault, M. (2005), S. 275. Vgl. auch ähnlich die Ausführungen bei SARTRE zum Konzept des Subjektivismus: „Das Wort Subjektivismus hat zwei Bedeutungen [...]. Subjektivismus bedeutet einerseits die Wahl des individuellen Subjekts durch sich selbst und andererseits die Unmöglichkeit für den Menschen, die menschliche Subjektivität zu überschreiten.“ (Sartre, J.-P. (2009), S. 322). 
Interpretation des Zugrundeliegenden einen Wandel durchlaufen hat ${ }^{82}$, wie die nachfolgenden Ausführungen zeigen.

Nach KIBLE lassen sich für die Antike sowie das Mittelalter insgesamt drei dominierende Begriffsverständnisse des Terminus „Subjekt“ feststellen. Neben dem heute noch (auch im Deutschen) üblichen logischen bzw. grammatikalischrelationalen Gebrauch ${ }^{83}$ (z. B. auch úлокєí $\mu \varepsilon v o v$ bei ARISTOTELES ${ }^{84}$ ) findet sich zweitens auch die Nutzung als Bezeichnung für ein Thema - wie dies z. B. auch heute noch im Englischen bzw. Französischen als „subject“ oder „sujet" geläufig ist. Darüber hinaus kann, und dies erscheint gerade für den philosophisch-fachwissenschaftlichen Gebrauch von zentraler Relevanz, der Terminus als ontologische Eigenschaftszuschreibung aufgefasst werden ${ }^{85}$ Insgesamt zeigt sich damit in der Zusammenschau, wie in der Literatur vielfach betont wird, dass das damalige Begriffsverständnis der heutigen Auffassung als „Objekt“ recht nahekommt. ${ }^{86}$ In diesem Sinne betont auch KÖRTNER: „Ursprünglich entsprach der Subjektbegriff also eher unserem heutigen Begriff des Objekts." ${ }^{\text {87 }}$ Ebenso heben auch HuCHLER, VOß und WeIHRICH hervor, dass mit dem antiken bis mittelalterlichen Subjektbegriff eher ,eine Art abhängige oder zumindest passive Größe gesehen [wird], die formenden Bestimmungen unterliegt (und unterliegen muss), ontologisch wie epistemologisch.“ ${ }^{\circ 8}$

\footnotetext{
${ }^{82}$ In Bezug auf die Wandlung des philosophischen Begriffsverständnisses betonen FETZ, HAGENBUCHLE und SCHULZ: ,Subjektivität, wie sie hier verstanden wird, meint in erster Linie das sich wandelnde Selbstverständnis des Menschen." (Fetz, R. L. / Hagenbüchle, R. / Schulz, P. (1998), S. V, vgl. auch Ornella, A. D. (2010), S. 162). Ebenso stellt auch REICHERTZ fest, ,dass jede Zeit andere Subjektvorstellung [sic!] und demnach auch andere Subjektformationen hat." (Reichertz, J. (2010a), S. 22).

${ }^{83}$ Zum logischen wie auch grammatikalischen Begriffsverständnis vgl. auch die klassischen Ausführungen bei Strawson, P. F. (2004).

${ }^{84}$ Vgl. Schnädelbach, H. (2004), S. 60.

${ }^{85}$ Vgl. Kible, B. (1998), Sp. 373. Zu einer ähnlichen Systematisierung vgl. auch Kirchner, F. et al. (2013), S. 637.

${ }^{86}$ Vgl. Neuser, W. (2013), S. 30 und S. 102.

${ }^{87}$ Körtner, U. H. J. (2011), S. 150. Vgl. ähnlich auch Neuser, W. (2013), S. 29. Dies hebt auch SAKAI hervor: „Im Blick auf die abendländische Philosophiegeschichte zeigt sich klar, daß der Begriff des ,subjectum“, also des ,hypokeímenon' seit Aristoteles durch das Mittelalter hindurch als ontologischer Kernbegriff gegolten hat. Seine Bedeutung war jedoch bekanntlich eine ganz andere, ja sogar entgegengesetzte als die heute gebräuchliche." (Sakai, K. (1994), S. 63).

${ }^{88}$ Huchler, N. / Voß, G. G. / Weihrich, M. (2007), S. 96.
} 
Mit dem Beginn der Neuzeit und insbesondere den Arbeiten René DesCARTES lässt sich jedoch eine deutliche Begriffsverschiebung des Terminus „Subjekt“ feststellen. ${ }^{89}$ So legte, obschon dieser noch prinzipiell das ältere Begriffsverständnis gebrauchte ${ }^{90}$, DESCARTES die Grundlagen für eine ontologische wie epistemologische Uminterpretation des Subjektbegriffs, welche auf die Begründung der autonomen Erkenntnisfähigkeit des Menschen fokussierte. Grundidee war hierbei, eine zugrunde liegende Substanz der Erkenntnis zu finden, welche in der Idee des cogito ergo sum ${ }^{91}$ als Vergewisserungsmöglichkeit bzw. fundamentum inconcussum der eigenen Existenz ${ }^{92}$ gipfelte und wiederum als Grundlage zur Klärung verschiedener Stufen des menschlichen Erkenntnisvermögens bei DESCARTES diente. Mit diesen fundamentalen Überlegungen entstand auch die bis heute wissenschaftshistorisch wirkmächtige Trennung zwischen einer geistigen Welt (von DESCARTES als res cogitans bezeichnet) und einer

${ }^{89}$ Vgl. Neuser, W. (2013), S. 30, welcher hervorhebt: „Bei Descartes verschiebt sich die Bedeutung im Begriff des Ich, das sich als denkendes Ich existent weiß, zum aktiven Subjekt als Akteur [...]." Vgl. auch Lumsden, S. (2014), S. 31; Schnädelbach, H. (2004), S. 61; Hagenbüchle, R. (1998), S. 4.

${ }^{90}$ Vgl. Gelhard, A. / Alkemeyer, T. / Ricken, N. (2013), S. 9. Vgl. auch Zima, P. V. (2017), S. 94-95 für eine Reflexion im Kontext neuerer philosophischer Strömungen.

${ }^{91}$ Diese Erkenntnis betont DESCARTES in unterschiedlicher Formulierung. In seinen Meditationes findet sich diese bspw. als „Ego sum, ego existo“ (Descartes, R. (2008), S. 48, in der Quelle ebenfalls herv.) und in seiner Principia philosophiae als ,ego cogito, ergo sum“ (Descartes, R. (2005), S. 14, in der Quelle ebenfalls herv.). Die oben aufgeführte, bekannte Formulierung findet sich am ehesten im französischen Original des Discours de la méthode als ,je pense, donc je suis“ (Descartes, R. (1987), S. 33, in der Quelle ebenfalls herv.). Erste Spuren dieser Überlegungen finden sich allerdings bereits bei AUGUSTINUS. Vgl. Augustinus, A. (1981), S. 498, welcher formulierte: ,Si enim fallor, sum. “Vgl. in diesem Zusammenhang auch Ornella, A. D. (2010), S. 93; Bürger, P. (2001), S. 35-38; Fischer, N. (1998), S. 340; Fuhrer, T. (1998), S. 324.

92 So betont auch HagenbUCHLE, dass der moderne Begriff des Subjekts ,im Sinne einer spezifischen Weise menschlicher Selbstvergewisserung verstanden werden" (Hagenbüchle, R. (1998), S. 4) kann. 
äußeren Welt der Dinge (res extens $a)^{93}$, welches paradigmatisch ${ }^{94}$ zu einer vielfach bis heute kontrovers diskutierten „Subjekt-Objekt-Spaltung“95 führte. ${ }^{96}$ Die Überlegungen von DESCARTES dienten nun wiederum als Grundlage für einen bis heute andauernden philosophisch-aufklärerischen Subjektdiskurs. Im Kontext hervorzuheben sind dabei z. B. die Beiträge von LEIBNIZ, bei welchem die modernen Subjekteigenschaften, z. B. im Rahmen seiner metaphysischen Schriften zur Monadologie, nun deutlich hervortreten. ${ }^{97}$ Auch KANT gebraucht den Subjektbegriff, führte dabei aber den Terminus des transzendentalen Subjekts ein, welches er vom empirisch wahrnehmbaren Subjekt unterschied, wobei ersteres als Wesenseigenschaft des Menschen gedacht wird, welche als existenzielle Voraussetzung jeglicher (empirischer wie nicht-empirischer ${ }^{98}$ ) Erkenntnis

\footnotetext{
${ }^{93}$ Vgl. Descartes, R. (2008), S. 38 und 56.

${ }^{94}$ SCHERER und MARTI bezeichnen dieses vorherrschende Paradigma auch als „SubjektObjekt-Modell“ (Scherer, A. G. / Marti, E. (2019), S. 21). Dieses hat in der Tat im wissenschaftlichen Kontext eine zentrale ideengeschichtliche Bedeutung und spiegelt sich folglich auch in grundlegenden wissenschaftstheoretischen Überlegungen wider, so z. B. in Welt 1 und 2 des bekannten Drei-Welten-Modells nach PopPER. Vgl. Popper, K. R. / Eccles, J. C. (1981), S. 38; Popper, K. R. (1980), S. 143-145; Popper, K. R. (1979), S. 74 und S. 106-107. Ähnliche Überlegungen finden sich auch bereits früher, z. B. bei Frege, G. (1986), S. 40-44. Die grundlegende Idee einer ontologischen Dreiteilung der Welt lässt sich bis in die klassische Antike zurückverfolgen. Vgl. Czichos, H. (2019a), S. 64. Zur Interaktion der drei Welten vgl. auch Eccles, J. C. (1994), S. 3. Auch in der Rechtspraxis ist eine Differenzierung zwischen Subjekten (häufig als „Personen“ bezeichnet) und Objekten geläufig. Diese Überlegungen finden sich auch in KANTs Rechtslehre. Vgl. Kant, I. (1968d), S. 223. Zur Diskussion des inhaltlich verwandten Begriffs der (moralischen) Person vgl. Abschnitt 4.2.3.

${ }^{95}$ Diese Bezeichnung stammt von KARL JASPERS. Vgl. Jaspers, K. (1994), S. 25. Vgl. auch Schnädelbach, H. (2004), S. 68-69 sowie die detaillierten Ausführungen bei Rosenthal, K. (1970) zur Diskussion des Problems und existierenden Ansätzen zur potentiellen Überwindung einer solchen Spaltung.

96 Vgl. einführend Zima, P. V. (2017), S. 1; Jörissen, B. / Meyer, T. (2015), S. 11-12; Kaehler, K. E. (2010), S. 97-98; Schnädelbach, H. (2004), S. 61-62; Simon, J. (1998), S. 762-764; Frank, M. (1988), S. 20.

${ }^{97}$ Vgl. z. B. Leibniz, G. W. (2002), S. 122 und S. 130. Vgl. einführend auch Neuser, W. (2013), S. 35-36; Kaehler, K. E. (2010), S. 94-117.

${ }^{98}$ Analog $\mathrm{zu}$ den grundlegenden wissenschaftstheoretischen Überlegungen in Abschnitt 2.5.1 sind in diesem Zusammenhang auch bei KANT bspw. (apriorische, also vor der empirischen Erfahrung liegende) Erkenntnisse formaler (z. B. mathematisch-logischer) oder ethischer Natur zu nennen.
} 
gedacht werden muss. ${ }^{99}$ Der Subjektbegriff wird damit nach NeusER zum fundamentalen „Prinzip des Denkens schlechthin.“100 Mit HEGEL wurde dann, wie in der Literatur vielfach pointiert hervorgehoben wird, der „Höhepunkt europäischer Subjektphilosophie“101 erreicht, die sich im Ausspruch „daß die Substanz wesentlich Subjekt ist“"102 äußerte, wobei wiederum eine begriffliche Nähe zu den klassischen Substanzüberlegungen von DESCARTES offenkundig wird. ${ }^{103}$

\subsubsection{Klassische subjektbasierte Begründungselemente der Verantwortungsfähigkeit}

Wie die vorausgegangenen Ausführungen zeigen, lässt sich bereits mit der frühen Aufklärung eine Begriffsverschiebung feststellen, wodurch im ontologischen wie epistemologischen Sinne die Grundlage zur Bestimmung eines autonomen, selbstbewussten wie auch verantwortlichen Wesens entstand. Dieser neue Begriff des Subjekts wird schließlich zum „philosophische[n] Prinzip der Neuzeit.“104 Betrachtet man nun den Kern ${ }^{105}$ der subjektphilosophischen Diskussion der Aufklärung, so wird deutlich, dass trotz aller Diskurse im Detail der Subjektbegriff in diesem Zusammenhang klar auf ein spezifisches humanistisches Menschenbild fokussiert, das den Menschen als autonomes, erkenntnisfähiges sowie reflexivvernünftiges Wesen auffasst. So betonen auch BEER und GRUNDMANN: „Das Subjekt, so wie es heute in den Sozial- und Geisteswissenschaften verhandelt

\footnotetext{
${ }^{99}$ Vgl. Kant, I. (1968c), B 404. Bei KANT wird das Subjekt (der reinen Vernunft) folglich zum „Prinzip aller Erkenntnis a priori“ und „Grund aller Erfahrung“ (Kaehler, K. E. (2010), S. 249). Vgl. einführend auch Zima, P. V. (2017), S. 101-102; Neuser, W. (2013), S. 2837; Kaehler, K. E. (2010), S. 209-212 und S. 246-265; Lambrecht, J. (2009), S. 204-229; Kobusch, T. (1998), S. 768-769; Simon, J. (1998), S. 767-774. Vgl. auch Frank, M. (1986), S. 28-29, der die Begriffe ,reines“ und ,empirisches Subjekt“ verwendet. Vgl. ähnlich Herrmann, F.-W. v. (2004), S. 42.

100 Neuser, W. (2013), S. 30.

${ }^{101}$ Frank, M. (1986), S. 15. Vgl. auch Neuser, W. (2014), S. 6.

102 Hegel, G. W. F. (2015), S. 17.

103 Vgl. Neuser, W. (2013), S. 30.

${ }^{104}$ Kaehler, K. E. (2010), S. 16. Auch DUSING hebt die „Subjektivität als Prinzip der Ethik“ (Düsing, K. (2005), S. 129) hervor.

105 Die Relevanz des Einnehmens einer Metaperspektive betont auch REICHERTZ: „Wie kann man die Geschichte des Subjekts (oder gar die Geschichte der Vernunft des Subjekts) schreiben? Zum einen, indem man die Geschichten vom Subjekt historisch aufarbeitet und kartographiert, zum anderen, indem man eine metatheoretische allgemeine Struktur des Subjekts versucht zu entwerfen.“ (Reichertz, J. (2010a), S. 40).
} 
wird, ist als ein Kind der Aufklärung mit dem Erbe dieser Tradition behaftet. Das ,Subjektive" ist allgemein durch die Fähigkeit zu einem rationalen Denken und einer Reflexion gesellschaftlicher und kultureller Umstände charakterisiert. Das Subjekt gilt als handlungsfähiges Individuum, das befähigt ist, seine eigenen und die gesellschaftlichen Verhältnisse nach Maßgabe der Vernunft zu gestalten.“106 Dabei wird das Subjekt in diesem Begriff zu einer humanistisch geprägten Potentialzuschreibung des Menschen mit, wie SCHNÄDELBACH hervorhebt, seinen „,kognitiven und praktischen Fähigkeiten“107 und ist nach VEITH Ausdruck des menschlichen ,Selbst- und Daseinsverständnisses in der Moderne.“108 Folglich wird auch, wie FRANK betont, seit der ,Sattelzeit ${ }^{* 109}$ (ca. 1750-1850) jedes menschliche Individuum als Subjekt angesehen. ${ }^{110}$ Im Kern können hierbei drei zentrale, aufeinander aufbauende Elemente dieses aufklärerischen Subjektbegriffs identifiziert werden.

$\mathrm{Zu}$ Beginn steht ein ontologischer Begriffsbestandteil, der auf der Eigenschaft des (Selbst-)Bewusstseins des Subjekts als besonderem Seinszustand (,Substanz“) rekurriert, welche dieses wiederum von Gegenständen differenziert. Diese grundlegende Perspektive auf das Subjekt wird vielfach in der Literatur hervorgehoben, welches in Begriffen wie der Faktizität eines Bewusstseins, IchErleben bzw. Selbstbezüglichkeit oder Identitätsbewusstsein deutlich wird. ${ }^{111}$ So

106 Beer, R. / Grundmann, M. (2004), S. 1. Auch ZIMA stellt heraus, dass das individuelle Subjekt als verantwortungstragende und autonome Instanz einen Begriff der Moderne darstellt, welcher sich von zuvor archaischen und feudalistischen Kulturen abgrenzt, die stärker durch Kollektivismus geprägt waren. Vgl. Zima, P. V. (2017), S. 4-5.

${ }^{107}$ Schnädelbach, H. (2004), S. 61.

108 Veith, W. (2018), S. 3. So hebt auch FRUCHTL hervor: „Über das Subjekt in seinen semantischen Variationen - als Ich, Ego, Selbst oder Individuum - nachdenken heißt über die Moderne nachdenken.“ (Früchtl, J. (2013), S. 79, in der Quelle ebenfalls herv.). Auch ALKEMEYER, BUDDE und FREIST betonen: „Subjekt- und Selbstkonzepte gehören zu den Grundkategorien der Selbstwahrnehmung, -reflexion und -deutung der modernen Gesellschaft.“ (Alkemeyer, T. / Budde, G. / Freist, D. (2013), S. 10). Analog hebt auch VEITH hervor, dass ,die Vorstellung ,Subjekt“ zu sein, dem Selbstverständnis moderner Menschen entspricht [...].“ (Veith, H. (2004), S. 9). Aus gesellschaftspolitischer Perspektive stellt zudem OELMÚLLER fest: „Mit dem Begriff des Subjekts bezeichnet man seit dem Ende des 18. Jahrhunderts die Einheit der vor allem neu entwickelten wirtschaftlichen, sozialen, politischen, rechtlichen, sittlichen, ästhetischen, geistigen und religiösen Freiheitsmöglichkeiten des modernen Menschen." (Oelmüller, W. (1994), S. 29).

${ }^{109}$ Dieser Begriff geht auf KosELLECK zurück, vgl. Karthaus, U. (2015), S. 26; Reichertz, J. (2010b), S. 185 sowie grundlegend Koselleck, R. (1972), S. XV.

${ }^{110}$ Vgl. Frank, M. (1988), S. 7; Frank, M. (1986), S. 17.

111 So stellt auch KIESELHORST heraus: „In diesem Sinne wird unter dem Subjekt das ,Ich“, das ,Bewusstsein “, der ,menschliche Geist“ verstanden [...]. “ (Kieselhorst, M. (2010), S. 40). 
beschreibt auch WILS das Selbstbewusstsein als „Zentrum der Subjektivität“112. Hieran anschließend lässt sich mit KNOBLAUCH hervorheben, dass „Wissen in Bewußtseinsprozessen gründet. Grund aller Wissensprozesse und Ausgangspunkt aller Handlungen ist das Subjekt." 113 In diesem Sinne subsumiert ROTH die aufklärerische Substanzauffassung vom Subjekt: „Das Ich ist der Mittelpunkt und Träger aller geistigen, emotionalen und willentlichen Akte [...]." ${ }^{\text {114 }}$ Bereits der Bewusstseinszustand ist dabei aus ethischer Perspektive zentral für die Verantwortungsfähigkeit, wie auch DUUsING herausstellt: ,Jede ethische Übernahme von Verantwortung für eine geschehene Tat, jede ethische Überlegung und Entscheidung $[\ldots]$ verlangt ein klares Bewußtsein der Person von sich und von ihrer Identität in verschiedenen Phasen der Erlebniszeit.“"115

Basierend auf den vorigen Überlegungen kann ein epistemologischer Bestandteil des Subjekts identifiziert werden ${ }^{116}$, welcher aufbauend auf einem Bewusstseinszustand als Erkenntnissubjekt diskutiert wird und z. B. die Verarbeitung und Reflexion von Sinnesdaten umfasst sowie formale oder moralische Reflexionsfähigkeiten einschließt. Im Kern des epistemologischen Subjektbegriffs steht folglich der Mensch als erkenntnisfähiges Wesen ${ }^{117}$, mit der Fähigkeit vernunftbasiert $^{118}$ zu denken und zu begreifen, d. h. (begründet) Begriffe über sich und die umgebende Welt zu entwickeln. In diesem Sinne konstatiert auch POSER: „Wird

Analog begreift auch FRANK „Subjektivität als Bewußtsein vom Ich“ (Frank, M. (1986), S. 26). Vgl. in diesem Kontext auch die Überlegungen bei RECKWITZ, welcher betont: „Die Identität des Subjekts ist damit gleichbedeutend mit dem was häufig auch das ,Selbst' (, self') genannt wird.“ (Reckwitz, A. (2012b), S. 17).

112 Wils, J.-P. (1997), S. 40.

113 Knoblauch, H. (2004), S. 38.

${ }^{114}$ Roth, G. (2001), S. 324. Die Kernauffassung des Subjekts als Bewusstseinszustand ist dabei ebenso in der angelsächsischen Literatur präsent und findet sich z. B. auch bei Oksenberg Rorty, A. (1991), S. 92.

115 Düsing, K. (2005), S. 133. Ähnlich betont auch KIEPAS: „Um verantwortlich sein zu können, muss man notwendig ein bewusster Verursacher sein, also ein bewusstes Subjekt, das die Wirkungen und Folgen seiner Handlungen vorhersehen und beurteilen kann." (Kiepas, A. (2010), S. 76-77).

116 So betont auch HAGENBUCHLE: „Vom Subjekt als ontologischem Substrat zu unterscheiden ist - besonders seit Descartes - das erkenntnistheoretische Subjekt." (Hagenbüchle, R. (1998), S. 4).

117 Zum Begriff der Erkenntnis vgl. die Ausführungen bei Gabriel, M. (2016), S. 159.

${ }^{118}$ So beschäftigt sich z. B. ADORNO mit den ,Apriori der erkennenden subjektiven Vernunft“ (Adorno, T. W. (1972a), S. 15). Auch RECKWITZ hebt hervor: „Das klassische Subjekt erhält seinen Kern in bestimmten mentalen, geistigen Qualitäten, die zugleich Ort seiner 
etwas erkannt, setzt dies nicht nur einen Erkenntnisgegenstand, das Objekt, voraus und eine Darstellung des Erkannten, etwa in einer Aussage, sondern auch ein erkennendes Subjekt.“"119 Neben der expliziten Bezeichnung als „Erkenntnissubjekt ${ }^{\text {"120 }}$ ist dabei auch die bereits aufgezeigte adjektivierte Bezeichnung als „erkennendes Subjekt" ${ }^{\text {"121 }}$ geläufig, auf welches z. B. KNOBLAUCH, ganz in der Tradition von DeSCARTES und Hegel, auch als „erkennende Substanz“122 rekurriert. Das Erkenntnissubjekt kann dabei Erkenntnisse aus einer Vielzahl von Domänen gewinnen und so auch, wie bereits im zweiten Kapitel dieser Arbeit ausgeführt, logische, empirische, metaphysische, aber auch normative Aussagen bilden und anhand der dort aufgezeigten Kriterien prüfen. Im Rahmen der Ethik ist, wie gezeigt wurde, insbesondere der Gebrauch normativer Aussagen (ethisch-normativ sowie deskriptiv begründet) von zentraler Bedeutung. Der souveräne Umgang mit diesen wird vielfach auch als die Fähigkeit des Einnehmens eines ,moral point of view" ${ }^{\prime 23}$ bezeichnet, also die Einsichtsfähigkeit in und die Begründungsfähigkeit von Normen, welche neben einer teilweise postulierten Moralintuition im Laufe der Sozialisation fraglos auch erlernt werden muss, und generell als weiteres zentrales Element der Verantwortungsfähigkeit eines Subjekts gilt.

Abschließend kann, aufbauend auf den zuvor dargestellten Ebenen, die Fähigkeit des Subjekts als Akteur hervorgehoben werden, also die potentielle Möglichkeit, nicht nur als bewusstes Wesen zu erkennen, sondern diese Erkenntnisse zu nutzen, um einen eigenen Willen zu entwickeln und diesen dann potentiell in konkreten Handlungen (d. h. intentionales Tun) umzusetzen, so

Rationalität sind. Ihm werden im klassischen Diskurs in diesem Sinne universale, allgemeingültige Eigenschaften - seien diese in der Vernunft oder einer Natur begründet - zugeschrieben." (Reckwitz, A. (2012b), S. 12).

${ }^{119}$ Poser, H. (2012), S. 31, in der Quelle ebenfalls herv.

${ }^{120}$ Vgl. z. B. Härle, W. (2018a), S. 196-197; Buller, A. (2002), S. 160; Ingarden, R. (1996), S. 252. Vgl. ähnlich auch Roth, G. (2001), welcher dieses als „Erlebnis-Subjekt“ bezeichnet (S. 326).

${ }^{121}$ Vgl. z. B. Scherer, A. G. / Marti, E. (2019), S. 21; Lauer, J. (2017), S. 104; Poser, H. (2016), S. 135; Kunneman, H. (1991), S. 23; Hartmann, N. (1965), S. 17. Vgl. auch klassisch Schopenhauer, A. (1988), S. 246; Horkheimer, M. (1937a), S. 13.

122 Knoblauch, H. (2004), S. 37. Vgl. auch Schütt, R. F. (2000), S. 1.

${ }^{123}$ Vgl. Arnold, D. G. / Beauchamp, T. L. / Bowie, N. E. (2020), S. 33-34; Gibbs, J. C. (2019), S. 3; Ulrich, P. (2016a), S. 59-60; Newton, L. (2013), S. 19; Gampel, E. H. (2009), S. 52; Frankena, W. K. (2004), S. 141. Der Begriff wurde stark durch KURT BAIER geprägt, welcher ihn im wissenschaftlichen Diskurs popularisierte. Vgl. Neuhäuser, C. (2011a), S. 64; Fry, S. T. (1990), S. 21 sowie konzeptionell grundlegend Baier, K. (1974), S. 185-213. 
dass in diesem Kontext die klassischen Begriffe der Willens- und Handlungsfreiheit ${ }^{124}$ bedeutsam sind. Neben der expliziten Bezeichnung als ,handelndes Subjekt“"125 oder „Handlungssubjekt“"126 findet sich auch dieser Begriffsbestandteil in der fachwissenschaftlichen Literatur in einer Vielzahl von Bezeichnungen. So beschäftigt sich z. B. DưsING mit der ,reine[n] praktische[n] Subjektivität“"127, woraus sich wiederum das ,tätige ethische Subjekt [...] als Fundament der Ethik entwickeln“128 ließe. Auch VEITH diskutiert das Konzept des „handlungsfähigen Subjekts“"129, welches bei PANNENBERG als „Selbständigkeit des Individuums als Subjekt" ${ }^{* 130}$ untersucht wird, während sich bei ZIMA eine Vorstellung vom

${ }^{124}$ Der Begriff der Freiheit ist von zentraler Bedeutung für die Ethik, insbesondere hinsichtlich der Begründung von Verantwortung. So betont auch QUANDT: „Das philosophische Letztprinzip von Moralität ist Freiheit, hierauf gründet die klassische Ethik." (Quandt, J. H. (2013), S. 94). Vgl. auch die Überlegungen bei PIEPER, welche das ,Prinzip der Moralität als Freiheit" (Pieper, A. (2017), S. 41) auffasst. Insbesondere die Willensfreiheit wird vielfach als elementare Voraussetzung für Handlungsfreiheit gesehen. So ist es auch nach DUSING die „Willensfreiheit, worin Handlungsfreiheit gründet.“ (Düsing, K. (2013), S. 211). Vgl. konzeptionell auch Fenner, D. (2020), S. 204-208. Vgl. auch die Ausführungen bei PLUMACHER, welcher konstatiert: ,Auf die Fähigkeit zur Reflexion ist auch die, Willensfreiheit‘ zurückzuführen, d. h. die Fähigkeit eines Subjekts, seine spontanen Wünsche, Interessen oder Ängste zu überdenken und seinem Handeln ein Ziel zu geben, das verantwortet [...] werden kann.“ (Plümacher, M. (2004), S. 95). Auch SOMBETZKI stellt fest: „Die Bedingungen dafür, dass jemand in diesem Sinne verantwortlich sein kann, sind [..] Handlungsfähigkeit sowie Urteilskraft [...].“ (Sombetzki, J. (2014), S. 32). Vgl. auch die Überlegungen bei WeISCHEDEL, welcher konstatiert: „Voraussetzung der Verantwortung ist also, daß der Mensch nicht durch anderes zu seiner Tat bestimmt wurde, sondern daß er selbst causa seines Tuns ist." (Weischedel, W. (1972), S. 19). Vgl. einführend zur Willens- und Handlungsfreiheit auch Beckermann, A. (2017). Zum Begriff der Freiheit vgl. auch die Ausführungen bei Ladwig, B. (2011) sowie die Überlegungen bei Küpper, H.-U. (1988), S. 318 zur Wahlfreiheit.

125 Vgl. z. B. Ornella, A. D. (2010), S. 92; Quitterer, J. (2008), S. 51; Harböck, W. (2006), S. 42; Schweppenhäuser, G. (2006), S. 66; Wetzel, M. (2004), S. 181; Kleinhans, B. (1999), S. 39; Köhler, H. (1997), S. 344.

126 Vgl. z. B. Dettinger, F. (2015), S. 108; Kellerwessel, W. (2007), S. 10; Kim, S. S. (2003), S. 57; Wils, J.-P. (2001), S. 59; Hubig, C. (1995), S. 61.

${ }^{127}$ Düsing, K. (2005), S. 151.

${ }^{128}$ Düsing, K. (2005), S. 151.

${ }^{129}$ Veith, H. (2004), S. 24.

${ }^{130}$ Pannenberg, W. (1996), S. 408. 
Subjekt als „handelnde und sprechende Instanz“131 bzw. als „Aktanten“132 wiederfindet. Schließlich stellt auch im sozialwissenschaftlichen Kontext REICHERTZ

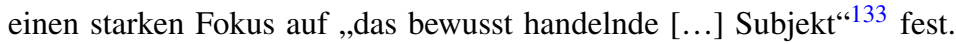

Die bisherigen Ausführungen haben gezeigt, dass der philosophischaufklärerische Subjektbegriff, wie er bis heute wirkmächtig ist, im Kern in drei aufeinander aufbauende Stufen differenziert werden kann, welches ontologisch das (Selbst-)Bewusstsein, epistemologisch die Erkenntnisfähigkeit und schließlich im Akteurssinne auch die Möglichkeit zu Handeln umfasst. ${ }^{134}$ Der Begriff fokussiert damit auf den Menschen mit seinen ,kognitiven und praktischen Fähigkeiten." 135 Diese Eigenschaftszuschreibungen ermöglichen in einem ersten Schritt jedoch lediglich ein Potential zur Verantwortungsübernahme. Wie die Literatur, z. B. im Rahmen der Betonung des Controllability-Prinzips, vielfach betont, müssen darüber hinaus auch die empirischen Restriktionen, welchen sich das Subjekt als ethisch befähigte Entität gegenübersieht, beachtet werden. So muss neben dem aktuellen Wissensstand über die Folgen auch die hinreichend konkrete Handlungskausalität in Bezug auf deren Folgen Berücksichtigung finden, welche sich z. B. in Begriffen wie der Akteurs- bzw. Agenskausalität niederschlägt. ${ }^{136}$ Erst wenn diese Bedingung zusätzlich erfüllt ist, konstituiert sich im klassischen Sinne inhaltlich auch eine konkrete Verantwortungsrelation

131 Zima, P. V. (2017), S. 9.

${ }^{132}$ Zima, P. V. (2017), S. 9.

${ }^{133}$ Reichertz, J. (2014), S. 106, welcher zudem die dispositiven Fähigkeiten des Subjekts herausstellt.

${ }^{134}$ So definiert auch SCHNÄDELBACH in Anlehnung an Digel, W. et al. (1981), S. 211 das Subjekt ,als das erkennende, mit Bewußtsein ausgestattete, handelnde Ich, das auch Träger von Zuständen und Wirkungen ist [...]." (Schnädelbach, H. (2004), S. 60). Ebenso hebt auch SAAR hervor: ,,Subjekt” ist ein Einheitsbegriff. Er bezieht sich auf eine fühlende, denkende und handelnde Instanz." (Saar, M. (2011), S. 356). Vgl. auch die Klassifikation bei Schmidt, H. / Schischkoff, G. (1982), S. 675, welche zwischen einem epistemologischen und akteursbezogenen Subjektbegriff differenzieren. Vgl. ähnlich auch Schöndorf, H. (2010c), S. 477.

135 Schnädelbach, H. (2004), S. 61.

${ }^{136}$ Diese wird in der Literatur z. T. auch Vertretern einer reinen Naturkausalität als „Ereigniskausalität“ gegenübergestellt. Vgl. im Überblick O’Connor, T. (2018), S. 84-86; Beckermann, A. (2017), S. 133-138; Schulte-Ostermann, K. (2011), S. 37-44; Lowe, E. J. (2008), S. 3-11; Quitterer, J. (2008), S. 52; Runggaldier, E. (2007), S. 293-296; Clarke, R. K. (1996), S. 19-21 sowie zu einigen klassischen Überlegungen auch Schallenberg, M. (2008), S. 298-305. 
eines Verantwortungssubjekts, wie oben aufgezeigt. ${ }^{137}$ Diese Überlegungen fasst nochmals die nachfolgende Abbildung 4.2 zusammen.

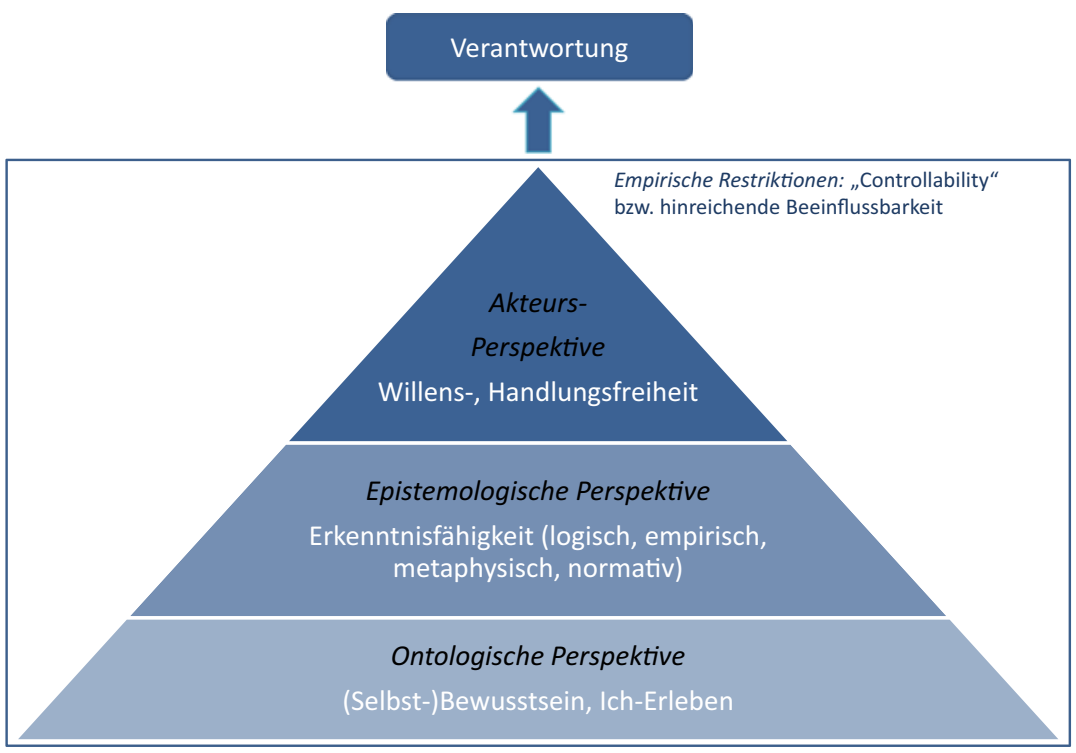

Abbildung 4.2 Ebenen des aufklärerischen Subjektverständnisses und darauf aufbauende klassische Verantwortungsbegründung

${ }^{137}$ Dabei ist zu beachten, dass eine Interaktion und gegenseitige Beeinflussung von Subjekt und Umwelt besteht. Während ersteres gestalterisch auf diese einwirken kann, nimmt die Umwelt wiederum Einfluss auf die Möglichkeiten der Entfaltung des individuellen Subjektpotentials. Gerade letzteres wird im Weiteren nochmals hinsichtlich seiner Implikationen durch die globalisierte Wissensgesellschaft diskutiert. Darüber hinaus muss das Subjekt aber auch die Möglichkeit der Wahl verschiedener Handlungen besitzen, wie PICHT hervorhebt: „Von Verantwortung kann nur gesprochen werden, wo es einen Spielraum verschiedener möglicher Handlungen gibt [...].“ (Picht, G. (2004), S. 323). 


\subsubsection{Abgrenzung zum verwandten Begriff der moralischen Person}

Neben dem, gerade im deutschsprachigen Raum, geläufigen Begriff des (moralischen) Subjekts existieren auch weitere, im Kontext der Aufklärung inhaltlich verwandte Begriffe wie Individuum oder Person. ${ }^{138}$ Im Rahmen dieser Arbeit sei dabei noch kurz auf einen dem aufklärerischen Subjektbegriff in seiner normativen Interpretation sehr nahestehenden Begriff kurz eingegangen: der (moralischen) Person. Ähnlich wie im Zusammenhang der Ausführungen zum Subjektbegriff bereits aufgezeigt, existiert auch hinsichtlich eines philosophischen Begriffs der Person kein inhaltlicher Konsens. So stellt auch SPAEMANN fest: „Der Sinn des Wortes ,Person“ ist wie der kaum eines anderen Wortes vom Kontext abhängig.“139 Ähnlich bemerkt auch BIRNBACHER: „Der Personenbegriff wird in der Philosophie in einer verwirrenden Vielfalt von Bedeutungen verwendet.“140 Schließlich betont auch MERRILL: „Personhood has been hotly debated throughout modern history [...]. The term ,person' is most often used in ways that introduce ambiguity rather than clarity into the practical concepts of contemporary debates about what being a person means." ${ }^{141}$ Neben der großen begrifflichen Heterogenität weisen auch viele Schriften darauf hin, dass sein etymologischer Ursprung bis heute nicht vollständig geklärt ist. ${ }^{142}$ Arbeiten, welche sich mit dessen Begriffsgeschichte beschäftigen, heben dabei jedoch vielfach hervor, dass sich der Begriff grundsätzlich schon im etruskischen phersu ${ }^{143}$ oder griechischen $\pi \rho$ ó $\sigma \omega \pi$ ov (prósopon) ${ }^{144}$ auffinden lasse. Hinsichtlich der deutschsprachigen Bezeichnung zeigt sich wiederum eine deutliche Nähe zum

\footnotetext{
${ }^{138}$ Eine Systematisierung dieser drei Begriffe findet sich bei Frank, M. (1986), S. 20-26.

139 Spaemann, R. (2006), S. 13. Auch KoBUSCH subsumiert: „,Kaum ein Begriff ist heute [...] so umstritten wie derjenige, der den eigentlichen Gegenstand der Metaphysik der Freiheit darstellt: der Begriff der Person." (Kobusch, T. (1997), S. 15). Vgl. auch die Formulierung bei QUANTE ,that there are no single notions of personal ,identity “, personhood or person [.]“ (Quante, M. (2007), S. 73-74).

${ }^{140}$ Birnbacher, D. (1997), S. 10.

${ }^{141}$ Merrill, S. A. B. (1998), S. 1. IKÄHEIMO spricht in diesem Zusammenhang sogar von einem ,slippery concept“ (Ikäheimo, H. (2007), S. 224).

142 Vgl. Wils, J.-P. (1997), S. 35.

${ }^{143}$ Vgl. Laux, L. / Renner, K.-H. (2008), S. 45; Rolnick, P. A. (2007), S. 11; Teichert, D. (2000), S. 92; Tafel, K. (1999), S. 233.

144 Vgl. Rolnick, P. A. (2007), S. 11; Dahrendorf, R. (2006), S. 28; Spaemann, R. (2006), S. 30; Teichert, D. (2000), S. 92.
} 
lateinischen Terminus persona ${ }^{145}$, welches von personare (,hindurchtönen“) ${ }^{146}$ oder per se sonans (,durch sich selbst tönend“) aber auch per se una (,durch sich selbst eine Einheit") ${ }^{147}$ stammen könnte. Diese Herleitung, insbesondere der ersten etymologischen Wurzel, korrespondiert auch mit dem Ursprung der antiken Begriffsverwendung, welcher sich ursprünglich auf eine Theatermaske bezog, die wiederum eine eingenommene Rolle repräsentierte ${ }^{148}$ - eine Verwendung, welche heute noch unter der Bezeichnung ,handelnde Personen“ vielfach in Beschreibungen von Theaterstücken aufzufinden ist. ${ }^{149}$ Neben dieser bis heute vorzufindenden terminologischen Verwendung nimmt der Personenbegriff eine zentrale Bedeutung in vielen weiteren Fachdisziplinen ein. So z. B. auch in der Rechtswissenschaft als Rechtssubjekt, der Soziologie im Kontext sozialer Rollen, der Psychologie in Bezug auf das Konstrukt „Persönlichkeit“"150 sowie der Theologie im Zusammenhang zur Trinität ${ }^{151}$, aber auch im grammatikalischen Kontext (als 1., 2., 3. Person Singular/Plural). ${ }^{152}$ Wie auch für das Subjekt bereits aufgezeigt, lässt sich im philosophischen Kontext analog zum Subjekt spätestens mit dem Einsetzen der Aufklärung ein zunehmender Gebrauch als Referenz für die besonderen Eigenschaften, aber auch einen besonderen Schutzstatus (,Würde“)

145 Vgl. Laux, L. / Renner, K.-H. (2008), S. 45; Reulecke, W. (2000), S. 485.

146 Vgl. Weihe, R. (2004), S. 181; Teichert, D. (2000), S. 92; Gründel, J. (1998), S. 160.

147 Vgl. Wils, J.-P. (1997), S. 36.

148 Vgl. Singer, P. (2011), S. 74; Laux, L. / Renner, K.-H. (2008), S. 45; Dahrendorf, R. (2006), S. 28; Weihe, R. (2004), S. 27. Vgl. auch die Ausführungen bei SPAEMANN, welcher zudem in diesem Zusammenhang die Relevanz der Differenzierung zwischen Rolle und Schauspieler hervorhebt: „Der Schauspieler ist Darsteller, er ist nicht das, was er darstellt.“ (Spaemann, R. (2006), S. 31, in der Quelle ebenfalls herv.).

${ }^{149}$ Vgl. Spaemann, R. (2006), S. 31.

150 Zum Begriff der Persönlichkeit vgl. auch die Ausführungen in Franken, S. (2019), S. 2733; Neyer, F. J. / Asendorpf, J. B. (2018), S. 2-22; Herzberg, P. Y. / Roth, M. (2014), S. 5-8; Smith, E. E. et al. (2007), S. 590-597; Roth, G. (2001), S. 341-349; Lersch, P. (1970), S. 7477 sowie mit philosophischem Fokus Quante, M. (2012), S. 155-157.

${ }^{151}$ In der Theologie lässt sich eine lange Begriffspraxis im Sinne einer Beschreibung der Trinität Gottes als drei Seinsweisen bzw. Personen auffinden (nach TERTULLIAN sinngemäß „una substantia - tres personae“, vgl. Tertullian (2001), S. 132-134 und S. 152), welche jedoch nur einem Gott zuzurechnen sind, womit wiederum eine Nähe zu dem bereits in der Antike existierenden Personenbegriff deutlich wird. Ähnlich stellte auch AUGUSTINUS in diesem Zusammenhang fest: ,tres personae sunt unius essentiae [...].“ (Augustinus, A. (2001), S. 274). Auch bei THOMAS VON AQUIN wurden diese Überlegungen wieder aufgegriffen und intensiv diskutiert. Vgl. hierzu z. B. Aquin, T. v. (2019), S. 196-216.

152 Vgl. zum Überblick auch Birnbacher, D. (2011a), S. 88-90; Sturma, D. (2008), S. 46-52; Spaemann, R. (2006), S. 13-14. 
des Menschen auffinden. ${ }^{153}$ Der Status als Person bzw. Personalität wird somit nach PANNENBERG zum „Inbegriff der Würde“154, woraus QUANTE schließt, dass „weitgehend unbestritten [sei], dass Personalität und Persönlichkeit mit einem ausgezeichneten ethischen Status verbunden sind.“155

Betrachtet man den Personenbegriff im Vergleich zum aufklärerischen Subjektbegriff, so lässt sich feststellen, dass manche Autoren, gerade im deutschsprachigen Raum, die Relevanz einer genaueren Begriffssystematisierung betonen ${ }^{156}$, wobei jedoch ersichtlicherweise kaum Konsens zur genauen Differenzierung besteht. Die vorigen Ausführungen, welche die Eigenschaften sowie den Schutzstatus des Menschen durch Würde hervorheben, mögen dabei den Eindruck erwecken, dass der Begriff der Person nun weiter aufzufassen sei ${ }^{157}$ als der des Subjekts, welcher häufig auch eher „technisch“ auf die Fähigkeiten des Menschen

153 Grundlegende Überlegungen finden sich allerdings, wie auch beim Subjektbegriff, bereits früher, so z. B. bei BOETHIUS, welcher Rationalität als Kerneigenschaft des Personenbegriffs auffasst. So definiert dieser Personen als „naturae rationabilis individua substantia“ (Boethius, A. M. S. (1988), S. 74 sowie S. 82). Vgl. auch die klassische Konzeption der ,persona moralis“"von PUFENDORF (Pufendorf, S. F. v. (2001), S. 16-20), welche in seinen Ausführungen sogar bereits Anwendung auf Kollektive findet. Vgl. zudem die moderne Definition bei KoBusch, welcher eine Person als „Wesen, das der Vernunft und Reflexion fähig ist [...]“ (Kobusch, T. (1998), S. 752-753) definiert. Analog subsumiert auch STURMA: „The concept of the person generally refers to someone who can act and react in the social space of reasons.“ (Sturma, D. (2007), S. 79). Ähnlich definiert auch SINGER die Person als ,a rational and self-aware being." (Singer, P. (2011), S. 75). Teilweise wird darüber hinaus auch die Kommunikationsfähigkeit als Element der Personeneigenschaft herausgestellt, so z. B. bei Sombetzki, J. (2014), S. 32 und S. 44-46; Kleinfeld, A. (1998), S. 157 sowie diskurstheoretisch bei Apel, K.-O. (1981), S. 400. Vgl. konzeptionell auch Edelmayer, E. (2012), S. 89-93.

154 Pannenberg, W. (1996), S. 407. Vgl. hierzu auch Schweidler, W. (2012), S. 121.

155 Quante, M. (2012), S. 163. So ist auch spätestens seit KANT der Personenbegriff zentral für die Begründung der Menschenrechte (vgl. Spaemann, R. (2006), S. 10; Kleinfeld, A. (1998), S. 168 und S. 188). Vgl. grundlegend auch Kobusch, T. (1997), S. 11. Menschenrechte werden in der Literatur auch teilweise zu „Personenrechten“ verallgemeinert, vgl. hierzu z. B. die Überlegungen bei Singer, P. (2011), S. 94-104; Spaemann, R. (2006), S. 13 und S. 252-264.

${ }^{156}$ In diesem Sinne betont z. B. PANNENBERG, dass trotz der faktisch vielfach synonymen Verwendung der Begriff der Person ,,bei genauerer Betrachtung [.] vom Subjektbegriff charakteristisch verschieden [sei], obwohl seine Geschichte auch zur Vorgeschichte des modernen Themas der Subjektivität gehört.“ (Pannenberg, W. (1996), S. 407).

157 So wird im Kontext des Personenbegriffs häufig hervorgehoben, dass der Begriff nicht nur deskriptiv, sondern auch normativ im Sinne bestimmter Rechte und Pflichten gebraucht wird. Vgl. z. B. Quante, M. (2012), S. 2; Birnbacher, D. (1997), S. 11. In diesem Sinne fomuliert auch GALLAGHER pointiert: „To have the status of moral personhood means two things: first, 
rekurriert. Allerdings zeigt die Literatur, dass, insofern eine Systematisierung intendiert wird, eher ein gegenteiliges Verhältnis der Begriffe vorgeschlagen wird, welches folglich den Personenbegriff enger als den des Subjekts erachtet. ${ }^{158}$ Ein klassisches Beispiel findet sich hierzu bei KANT, welcher den Personenbegriff als Teilmenge des Subjekts auffasst. In diesem Sinne wäre eine „Person [.] dasjenige Subjekt, dessen Handlungen einer Zurechnung fähig sind. Die moralische Persönlichkeit ist also nichts anders, als die Freiheit eines vernünftigen Wesens unter moralischen Gesetzen [...]." ${ }^{159}$ Auch SCHNÄDELBACH fokussiert auf Personen als spezifische Subjekte: „Das Subjekt in rechtlicher und moralischer Hinsicht ist [.] nichts anderes als die Person [...], die sich handelnd auf die gegenständliche Welt [.] beziehen [kann] und dafür Verantwortung zu übernehmen fähig ist [...].“160

Generell zeigt die Literaturdurchsicht, dass im Kontext des philosophischen Personenbegriffs besonders häufig die Fähigkeit zum reflexiv-intentionalen Handeln betont wird. So ist auch nach FRANKEN eine „Person ein handelndes Subjekt ${ }^{* 161}$. In einem erweiterten Sinne stellt aber auch analog KORSGAARD fest: „Eine Person ist sowohl ein Handlungssubjekt wie auch ein Subjekt

that one has the ability to take responsibility for one's actions, and second, that one ought to be treated in a certain way." (Gallagher, S. (2007), S. 199).

${ }^{158} \mathrm{Vgl}$. in diesem Zusammenhang auch die Ausführungen bei WILS, welcher Personalität als „Binnenaspekt von Subjektivität“ (Wils, J.-P. (1997), S. 41, in der Quelle ebenfalls herv.) erachtet.

${ }^{159}$ Kant, I. (1968d), S. 223, in der Quelle gesperrt herv. Diese Auffassung wird jedoch auch bei KANT nicht stringent durchgehalten. So definiert dieser an anderer Stelle mit starkem Bezug auf die Identität des Subjekts: „Daß der Mensch in seiner Vorstellung das Ich haben kann, erhebt ihn unendlich über alle andere auf Erden lebende Wesen. Dadurch ist er eine Person und, vermöge der Einheit des Bewußtseins bei allen Veränderungen, die ihm zustoßen mögen, eine und dieselbe Person [...].“ (Kant, I. (1980), S. 11, in der Quelle gesperrt herv.). Der Gedanke, den Begriff der Person über die Einheit und Beständigkeit des Bewusstseins zu konstituieren, findet sich bereits bei LOCKE als ,,thinking intelligent Being, that has reason and reflection, and can consider it self as it self, the same thinking thing in different times and places [...].“(Locke, J. (2008), S. 208). Auch STURMA hebt die „Identität des Selbstbewußtseins“" (Sturma, D. (2008), S. 148) als zentrales Charakteristikum von Personen hervor. Vgl. zudem die Überlegungen bei LUCKMANN, welcher die ,,[p]ersönliche Identität als grundlegende Daseinsform des Menschen“(Luckmann, T. (1996), S. 295) bezeichnet. Vgl. in diesem Zusammenhang auch Gerhardt, V. (2018), S. 294; Sombetzki, J. (2014), S. 51; Böhme, G. (2012), S. 11; Quante, M. (2012), S. 5-11; Quante, M. (2007), S. 59-63; Quante, M. (1999), S. 9-12; Kobusch, T. (1997), S. 18.

${ }^{160}$ Schnädelbach, H. (2004), S. 60. Vgl. auch die Ausführungen bei KLEINFELD, welche auf die „Person als moralisches Subjekt“ (Kleinfeld, A. (1998), S. 235) fokussiert.

${ }^{161}$ Franken, S. (2019), S. 27. 
der Erfahrung [...].“162 Schließlich betont SpAEMANN: „Personen sind Subjekte des Könnens"163. Allerdings finden sich auch Autoren, welche neben der epistemologischen bzw. Handlungsperspektive auch die ontologischen Substanzüberlegungen des Subjektbegriffs hervorheben. In diesem Sinne subsumieren z. B. IKÄHEIMO und LAITINEN wie folgt: „In philosophy, persons are typically thought of as being self-conscious, as having self-concern, second-order desires ${ }^{164}$, moral conscience, first-person perspective or other epistemic and practical, conscious or unconscious way of relating to their attitudes, emotions and actions, and to themselves as their subjects. " 165

Diese Übersicht verdeutlicht nun in der Zusammenfassung der Perspektiven, dass viele Beiträge zum philosophischen Begriff der Person doch die aufgezeigten Kerneigenschaften des aufgeklärten Subjektbegriffs widerspiegeln. ${ }^{166}$ Eine Analyse des angelsächsischen Sprachraums zeigt außerdem, dass dort grundsätzlich die Bezeichnung als „,moral person“ dominiert, während der Terminus des „,moral subjects" eher selten gebraucht wird. Darüber hinaus ist ersichtlich, dass gerade die aufgezeigten Subjekteigenschaften nicht nur eine klassische Argumentationskette zur Begründung von Verantwortung konstituieren, sondern ferner, aufgrund des besonderen Status einer solch potentiell frei reflexions- und handlungsfähigen Einheit, auch ein besonderer Schutz begründet werden kann, womit auch die

162 Korsgaard, C. M. (1999), S. 195.

163 Spaemann, R. (2002), S. 422.

${ }^{164}$ Der Begriff geht auf FRANKFURT zurück und bezieht sich auf die Selbstbestimmung des Willens (z. B. in dem Sinne, dass der, ,gute Wille“ (KANT) nur universalisierbaren Maximen folge) in Bezug auf konkrete ,first-order desires“. Die Fähigkeit zur Reflexion und Selbstbestimmung eines konkreten Willens ist nach FRANKFURT charakteristisch für Personen. Vgl. Frankfurt, H. G. (1971), S. 6-10.

165 Ikäheimo, H. / Laitinen, A. (2007), S. 10. Damit zeigen sich bereits hier klar alle drei zuvor aufgezeigten Ebenen des aufklärerischen Subjektbegriffs.

166 So definiert auch REULECKE den Begriff der Person in Referenz auf „den Menschen als geistiges, selbstbewußtes Einzelwesen, das im ausgewogenen Zusammenwirken seiner Eigenschaften, seines Denkens, Erlebens und Handelns zu freier, verantwortungsvoller Willensentscheidung fähig ist [...].“ (Reulecke, W. (2000), S. 485). Vgl. ähnliche Überlegungen auch in Quante, M. (2012), S. 1. Zudem stellt KoBUSCH die Auffassung vor, ,den durch die Metaphysik der Person ermöglichten Freiheitsgedanken im Sinne der Subjektivität als das eigentlich Neue des neuzeitlichen Denkens anzusehen." (Kobusch, T. (1997), S. 20). Analog subsumiert STURMA: „Im Fall des Begriffs der Person konkretisiert sich die Problematik des Subjektgedankens in der Frage, ob vernünftige Individuen in ihren Bewußtseinszuständen und Handlungsverläufen auf signifikante Weise selbst präsent sind." (Sturma, D. (2008), S. 25, in der Quelle ebenfalls herv.). Vgl. auch die Überlegungen bei Wils, J.-P. (1997), S. 26, welcher hervorhebt, dass nach einem kritischen Diskurs in der Subjektphilosophie sich die Diskussion nun auf den Personenbegriff übertragen habe. 
zweite Perspektive des Personenbegriffs wiederum abgedeckt erscheint. In diesem Sinne hebt auch NEUSER den vielfach synonymen Begriffsgebrauch sowie die ethischen Implikationen des Subjektstatus pointiert hervor: „An dem Subjekt $[\ldots]$ hängen die Freiheit des Einzelnen und die Würde des Menschen. In einer logischen Kontraktion werden in der Folge häufig Subjekt, Mensch, Individuum, Person und Einzelner äquivok gedacht.“167 Da die Literatur zudem, wie aufgezeigt, auch im Zusammenhang zum Begriff der Person eine große Heterogenität aufweist sowie im Rahmen dieser Arbeit eine weitere begriffliche Systematisierung zwischen moralischer Person und moralischem Subjekt keinen weiteren Erkenntnisgewinn verspricht, sei auf eine detailliertere Systematisierung verzichtet und der Auffassung gefolgt, Subjekt und Person im ethischen Sinne synonym $^{168}$ als Referenz für diejenigen Entitäten zu gebrauchen, welche verantwortungsfähig sind, aber andererseits regelmäßig durch diese Eigenschaften als moralisches Subjekt auch ein besonderer Schutzstatus zukommt.

Die aufgezeigten, in der Aufklärung begründeten Subjekteigenschaften sind jedoch seit dem 19. Jahrhundert zunehmend kritisch evaluiert worden, so dass sich die Frage stellt, inwiefern die zugrunde liegenden Annahmen noch tragfähig zur inhaltlichen Begründung der individualsubjektiven Verantwortungsrelation sein können. Dies ist Gegenstand des nachfolgenden Abschnitts.

\subsubsection{Der aufklärerische Subjektbegriff in der Kritik}

Die in den vorigen Ausführungen aufgezeigten Annahmen hinsichtlich des ontologischen, epistemologischen sowie Akteursstatus des Subjekts stellten dabei im 19. und 20. Jahrhundert noch vielfach den „Common Sense bezüglich dessen, was es heißt, ein ,Individuum“ oder ,Selbst` zu sein [...]"169 dar. Mit dem aufkommenden 19. Jahrhundert lässt sich jedoch eine diametrale Umdeutung des Subjektbegriffs konstatieren, welcher mit einer zunehmend kritischen Rezeption des aufklärerischen Subjektbegriffs korrespondiert sowie in Teilen gar zu einer vollständigen Infragestellung der Begriffsverwendung an sich führte. In diesem Zusammenhang seien nun zuerst kompakt einige philosophische Überlegungen der Nachmoderne ausgeführt, welche mit neueren Überlegungen

${ }^{167}$ Neuser, W. (2014), S. 8, in der Quelle ebenfalls herv. Vgl. in diesem Zusammenhang auch Neuser, W. (2017), S. 79 sowie Frank, M. (1986), S. 20 und S. 64.

168 Vgl. zu dieser weitverbreiteten Begriffspraxis auch z. B. Dietzfelbinger, D. (2015), S. 4849; Kiepas, A. (2010), S. 76-77; Wachendorff, E. (2005), S. 81-83.

${ }^{169}$ Reckwitz, A. (2012b), S. 12. 
zur Willensfreiheit aus neurobiologischer Perspektive abgerundet werden, bevor die Implikationen der Digitalisierung und Globalisierung auf die individuelle Verantwortungsträgerschaft in den Fokus gerückt werden.

\subsubsection{Klassische Kontroversen im subjektphilosophischen Diskurs}

Befasst man sich mit dem subjektphilosophischen Diskurs, welcher sich an die klassischen Überlegungen der Aufklärung anschließt, so lässt sich feststellen, dass im Laufe der Zeit eine zunehmende Begriffsverschiebung stattgefunden hat, welche das Subjekt nicht mehr zur Beschreibung des Menschen als autonomes Wesen gebraucht, sondern wieder stärker die zweite Begriffsinterpretation des Subjekts als „Unterworfenes“ aufgreift. Dabei finden sich bereits bei HEGEL Überlegungen, in welchen sich das Subjekt der Staatsgewalt - allerdings durchaus noch durch Einsicht in diese Notwendigkeit begründet - unterwirft. ${ }^{170}$ Diese (partielle) Freiwilligkeit wird jedoch seit dem 19. Jahrhundert, so z. B. von Marx, Freud, Nietzsche, Heidegger, Wittgenstein, Dewey, Foucault, RORTY, LYOTARD sowie DERRIDA hinter überaus verschiedenen konzeptionellen Annahmen zunehmend infrage gestellt. ${ }^{171}$ Übergreifend gilt dabei, dass diese ,den handelnden Subjekten nur noch geringe Autonomie zu[billigen], [sie] sahen sie als Resultat bzw. Exekutoren von extern (sozial) vorgegebenen Mustern: Der Mensch ist das Ensemble seiner gesellschaftlichen Verhältnisse (so Marx)." ${ }^{172}$ Aus dem philosophischen Diskurs haben sich, neben Überlegungen zu staatlichen Machtstrukturen, welchen sich Menschen zu unterwerfen hätten, auch weitere Kritikpunkte am aufklärerischen Subjektbegriff entwickelt. Ein intensiv diskutierter Aspekt fokussiert hierbei auf die der Aufklärung vorgeworfene Vernachlässigung der Sozialisation als Voraussetzung der Nutzung des Freiheitspotentials. In diesem Zusammenhang wird vielfach der Begriff der Subjektivierung gebraucht, welcher darauf abzielt, dass ein "Subjekt“ nicht von

\footnotetext{
${ }^{170}$ Vgl. Zima, P. V. (2017), S. 107 und S. 117 sowie zum Staatswesen nach HEGEL grundlegend Hegel, G. W. F. (2008), S. 398-410.

${ }^{171}$ Vgl. Reckwitz, A. (2012b), S. 12-13; Gloy, K. (2002), S. 125. FOUCAULT und RORTY kehren in ihren Spätwerken aber wieder zum aufklärerischen Subjektbegriff zurück. Vgl. z. B. Hagenbüchle, R. (1998), S. 18-19; Wetz, F. J. (1998), S. 1277.

${ }^{172}$ Schülein, J. A. (2000), S. 658. Diese Überlegungen finden sich bis heute in der neueren subjektphilosophischen Forschung, z. B. bei JUDITH BUTLER. Sie bezeichnet „,Subjektivation' [.] [als] den Prozeß des Unterworfenwerdens durch Macht und zugleich den Prozeß der Subjektwerdung." (Butler, J. (2001), S. 8).
} 
Beginn an bestehe, sondern durch Bildungsprozesse ${ }^{173}$ erst geschaffen werde, ${ }^{174}$ welches bei ALTHUSSER nach SAAR gar zur „Subjektproduktion“175 werde. In diesem Sinne stellen auch BEER und GRUNDMANN heraus: „Das Subjekt ist Subjekt, weil es in intersubjektiven Zusammenhängen via Sozialisation und Lernprozessen zu einem Subjekt wird.“176 Prägnant fasst auch BURGER in diesem Kontext die Überlegungen FouCAULTS zusammen ,daß wir nicht als Subjekte geboren, sondern zu Subjekten gemacht werden."177 Schließlich stellt auch RICKEN fest: „Dass Menschen nicht einfach Subjekte sind [...] ist eine - zumindest (sozial-)wissenschaftlich - weithin geteilte Überzeugung; die Frage jedoch, wie denn Menschen zu Subjekten werden [...] ist längst noch nicht beantwortet [...]. “178 In diesem Zusammenhang wird auch vielfach darauf hingewiesen, dass selbst das grundlegendste ,Werkzeug“ der Philosophie, die natürliche Sprache, nicht von Subjekten selbstkonstituiert, sondern schon immer bereits vorgefunden und daher geistig präformierend erlernt bzw. schlicht übernommen werde. In diesem Sinne finde ,der Mensch [.] die Sprache immer schon vor und könne sie nicht durch Eingriffe verändern." ${ }^{179}$ Damit ist nach VEITH auch ,,[d]as Subjekt der Sozialisationstheorie [..] nicht selbstgenügsam, sondern polyreferentiell - und

${ }^{173}$ Neben dieser externen Einflussnahme wird jedoch auch immer wieder der eigene, individuelle Einfluss während dieser Konstitution hervorgehoben. So summieren auch GELHARD, ALKEMEYER und RICKEN diese Auffassung: ,Subjekte [sind] nicht einfach , da ', sondern Produkt von Prozessen der Selbstbildung und Selbstformung [...].“(Gelhard, A. / Alkemeyer, T. / Ricken, N. (2013), S. 10). Ebenso stellt auch RICKEN heraus, dass mit dem Begriff der „Subjektivierung gegenwärtig im sozialwissenschaftlichen und -philosophischen Diskurs jener Prozess markiert [wird], in dem Menschen ebenso zu Subjekten gemacht werden wie sich selbst dazu machen.“ (Ricken, N. (2013), S. 31).

174 Ohne diese Überlegungen im Weiteren detailliert kritisch zu würdigen, sei in diesem Zusammenhang darauf hingewiesen, dass im Rahmen dieser Diskurse vielfach offenkundig schlicht ein anderes Begriffsverständnis des Subjekts zugrunde liegt, welches auf eine Realisierung der Subjektfähigkeiten, nicht jedoch auf das Subjektpotential als Eigenschaft selbst fokussiert. Vgl. hierzu auch Saar, M. (2013), S. 17.

175 Vgl. Saar, M. (2013), S. 18-20.

176 Beer, R. / Grundmann, M. (2004), S. 2.

177 Bürger, P. (2001), S. 27.

${ }^{178}$ Ricken, N. (2013), S. 29.

${ }^{179}$ Bürger, P. (2001), S. 16. Dies korrespondiert mit dem ,linguistic turn“ in der Philosophie bzw. der Hinwendung zur sprachanalytischen Philosophie. Vgl. hierzu einführend Leiss, E. (2012), S. 1-15; Janich, P. (2005), S. 26-39; Kanterian, E. (2004), S. 42-50; Haag, J. (2003), S. 89. 
genau dadurch unterscheidet sich der sozialisationstheoretische vom cartesianischen Subjektbegriff." ${ }^{180}$ KNOBLAUCH wiederum verschiebt im Kontext der poststrukturalistischen Philosophie schließlich den Fokus auf die reine gesellschaftliche Konstruktion des Subjekts. So werde das Subjekt nun nicht mehr „als feste, erkennende Substanz, sondern als Ergebnis diskursiver Konstruktionen angesehen [.].“181

Die Perspektive des Subjekts als Unterworfenes fasst schließlich VAN REIJEN in einem pessimistischen Resümee zusammen: „Die Subjekte sind tot, sie können nichts mehr produzieren, im Gegenteil, sie werden produziert. Wir sprechen nicht die Sprache - die Sprache spricht ... uns. Wir machen nicht die Tradition, die Tradition macht uns." ${ }^{\text {182 }}$ VAN REIJEN greift hier hinsichtlich der Formulierung erkennbar auf den vielfach postulierten „Tod des Subjekts“183, wie er als Begriff FOUCAULT $^{184}$ zugeschrieben wird, zurück und skizziert damit ein diametral zur Aufklärung verschiedenes Menschenbild, bei welchem die zentralen, vorherig unterstellten Eigenschaften, welche in der Autonomie und Verantwortlichkeit des Menschen gipfelten, zunehmend hinterfragt werden. So stellt HAGENBÜCHLE fest: „Im Gegensatz zur traditionellen Begriffsauffassung hat heute das Subjekt seine

\footnotetext{
${ }^{180}$ Veith, H. (2004), S. 9.

${ }^{181}$ Knoblauch, H. (2004), S. 37.

182 Reijen, W. v. (1988), S. 398.
}

${ }^{183}$ In diesem Sinne betont auch GLOY: ,Gegenwärtig erlebt der Subjektivitäts- und Selbstbewußtseinsbegriff erneute Aktualität, freilich in Form seiner Destruktion und Verabschiedung: ,Der Tod des Subjekts“ ist zum Schlagwort geworden.“ (Gloy, K. (2002), S. 125). Auch PAULus betont: „Der Tod des Subjekts scheint allgegenwärtig zu sein. Die abendländische Philosophiegeschichte lässt sich bis weit ins 20. Jahrhundert hinein in weiten Teilen als ein Diskurs über die Destruktion eines einst vermeintlich sich allmächtig dünkenden Subjekts lesen [...].“ (Paulus, M. (2009), S. 1). Ähnliche Formulierungen finden sich auch in der Literatur als „Verschwinden des Subjekts“ (z. B. bei Bürger, P. (2001); Mutschler, H.-D. (1994)) oder der „Auflösung des Subjekts in der Moderne“ (Seubert, H. (2005), S. 35).

${ }^{184}$ Vgl. Foucault, M. (1983), S. 462, welcher diese Terminologie jedoch nicht selbst gebraucht, allerdings im Verschwinden aufgefasste, klassisch zugeschriebene Subjekteigenschaften des Menschen andeutet. Die vielfach aufgegriffene Formulierung wiederum steht in erkennbarer Referenz zur bekannten Formulierung NIETZSCHES: „Gott ist todt [...].Wir haben ihn getödtet, ihr und ich! Wir Alle sind seine Mörder!“ (Nietzsche, F. (2013), S. 108 und S. 135) - eine Formulierung, wie sie sich ähnlich auch bereits bei HEINE findet: „Doch jetzt ist alles wie verschoben, / Das ist ein Drängen! eine Not! / Gestorben ist der Herrgott oben, / Und unten ist der Teufel tot.“ (Heine, H. (1893), S. 114). Im übertragenen Sinne hinterlässt auch nach BURGER das Subjekt eine Lücke, falls dieses als Paradigma eliminiert wird (vgl. Bürger, P. (2001), S. 18-19, vgl. auch Oelmüller, W. (1994), S. 45). Auch bei ERNST MACH findet sich mit dem Ausspruch „Das Ich ist unrettbar“ (Mach, E. (1991), S. 20, vgl. auch Foucault, M. (2004), S. 37) eine ähnliche Formulierung. 
Bedeutung als ,fundamentum inconcussum “ weitgehend verloren. Es ist jetzt [...] das ,Unterworfene', wie es schon von Althusser und neuerdings auch von Lévinas uminterpretiert worden ist." 185

Diese aufgezeigte, eher pessimistische Perspektive auf den Subjektbegriff ist dabei typisch für subjektphilosophische Werke der Postmoderne ${ }^{186}$, welche vielfach darauf rekurrieren, dass das Subjekt nicht mehr autonomer „Unterwerfer“, sondern selbst unterworfen oder gar am Zerfallen sei. ${ }^{187}$ So subsumiert auch ZIMA: „Die als postmodern bezeichneten Autoren [...] sprechen offen aus, was im literarischen Modernismus [...] nur anklingt: daß das individuelle Subjekt zerfallen oder verschwunden ist." ${ }^{188}$ Dies führt schließlich bei RENN zur pointierten Feststellung, dass ,[d] as individuelle Bewusstsein [.] nicht [mehr] Herr im eigenen Hause ${ }^{\star 189}$ sei.

Verschärft wird diese Auffassung noch durch jüngere neurowissenschaftliche Erkenntnisse, welche bis heute kontrovers diskutiert werden. ${ }^{190}$ So subsumiert REICHERTZ: „Eine Vielzahl von Neurowissenschaftlern/innen geht seit gut einem Jahrzehnt noch weiter: Sie verkünden in und mit den Medien lautstark das endgültige Ende des Subjekts [...]. Sie stellen dabei das Gehirn bzw. die Gehirnschaltungen als Urgrund und Ursprung menschlichen Tuns vor.“191 Diese Überlegungen beruhen vielfach auf neurowissenschaftlichen Untersuchungen zur Entscheidungsfindung. Eines der bekanntesten Experimente stammt in diesem Zusammenhang von BENJAMIN LIBET, welcher feststellte, dass das Aktivierungspotential zur Handlung dessen bewusster Repräsentation im Gehirn

\footnotetext{
185 Hagenbüchle, R. (1998), S. 78.

${ }^{186}$ Vgl. einführend Zima, P. V. (2016), S. 19-46. Wissenschaftsparadigmatisch kann in diesem Kontext z. B. auch Feyerabend, P. (1993), S. 9-19 hervorgehoben werden.

${ }^{187}$ Vgl. Zima, P. V. (2017), S. 55 und S. 193; Bürger, P. (2001), S. 203; Ricken, N. (1999), S. 127-130; Großmann, A. (1992), S. 65. In diesem Zusammenhang subsumiert auch ORNELlA: „In der Postmoderne geriet das Subjekt zunehmend ins Schussfeld der Kritik, nicht zuletzt wegen seines Absolutheitsanspruchs und seiner Autonomie." (Ornella, A. D. (2010), S. 79).

188 Zima, P. V. (2017), S. 193.

${ }^{189}$ Renn, J. (2012), S. 35. Eine ähnliche Formulierung findet sich auch bei FREUD, welcher im Rahmen seiner Psychoanalyse konstatierte, ,,daß das Ich nicht Herr sei in seinem eigenen Haus." (Freud, S. (1972b), S. 11, in der Quelle gesperrt herv.). Vgl. auch Roth, G. / Strüber, N. (2019), S. 282.

190 Allerdings wies bekanntermaßen bereits FREUD darauf hin, dass das menschliche Handeln nicht vollständig bewusst, sondern vielfach auch durch unterbewusste Prozesse gesteuert sei. Vgl. Freud, S. (1972a), S. 239-255; Freud, S. (1969), S. 83-86.

${ }^{191}$ Reichertz, J. (2006), S. 11. Vgl. ähnlich auch Reichertz, J. (2010a), S. 39.
} 
vorausgehe ${ }^{192}$, woraus zum Teil geschlussfolgert wurde, dass das Bewusstsein automatische Prozesse nur ex post "rationalisiere“ und folglich der freie Wille zu Handeln nur eine Illusion sei. ${ }^{193}$ Hierauf aufbauend wurde auch die bereits in der Philosophie über Jahrhunderte diskutierte Möglichkeit der menschlichen Willensfreiheit wieder aufgegriffen und im Sinne der Bestätigung eines biologischen Determinismus, so z. B. bei ROTH, SINGER oder PRINZ, interpretiert ${ }^{194}$, welcher folglich keinen Raum mehr für echte Willensfreiheit lässt. NOLLMANN fasst diese Überlegungen wie folgt zusammen: ,Viele halten angesichts der messbaren neuronalen Regelmäßigkeiten gar die Freiheit des menschlichen Willens insgesamt für eine widerlegte Illusion." ${ }^{195} \mathrm{Er}$ stellt in diesem Kontext die Frage, inwiefern dann ,auch das sinnhaft kommunizierende Subjekt, das seinen Handlungen Bedeutungen beimisst, am Ende"196 sei. Ähnlich interpretiert auch RоTH diese neurowissenschaftlichen Befunde: „Die Wirklichkeit ist nicht ein Konstrukt meines Ich, denn ich bin selbst ein Konstrukt. Vielmehr geht ihre Konstruktion durch das Gehirn nach Prinzipien vor sich, die teils phylogenetisch, teils frühontogenetisch entstanden sind und ansonsten den Erfahrungen des Gehirns mit seiner Umwelt entstammen. Diese Prinzipien sind meinem Willen nicht unterworfen.

\footnotetext{
192 Vgl. Libet, B. (1999), S. 47-51; Libet, B. (1985), S. 530-534.

193 So stellt ROTH fest: „Wir müssen also davon ausgehen, dass das Gefühl, dass wir das, was wir jetzt tun, kurz zuvor gewollt haben, ebenso eine Täuschung ist wie die Annahme, dass dieser Willensakt die Tat ursächlich bedingt." (Roth, G. (2001), S. 443, in der Quelle ebenfalls herv.).

${ }^{194}$ In diesem Sinne könne es nach RоTH ,keinen vernünftigen Zweifel daran geben, dass es auch bei den hochstufigen Prozessen in unserem Gehirn, die für die Steuerung unseres Verhaltens zuständig sind, deterministisch zugeht, dass also weder so etwas wie ein freier Wille noch quantenphysikalisch indeterministische Prozesse hierbei eine Rolle spielen." (Roth, G. (2001), S. 447). Ähnlich stellt SINGER fest: „Auch wenn dabei einige der Variablen ins Bewußtsein dringen und wir die resultierenden Entscheidungen dann als frei gefällte wahrnehmen, bleibt festzuhalten, daß auch die bewußten Deliberationen auf neuronalen Prozessen beruhen und somit deterministischen Mechanismen gehorchen müssen." (Singer, W. (2004), S. 116). Vgl. auch die Ausführungen bei Prinz, W. (2012), S. 175-180.

195 Nollmann, G. (2006), S. 63. Auch BEER und GRUNDMANN subsumieren den neurowissenschaftlichen Diskurs und stellen heraus, dass in dieser Perspektive „der Mensch zu einem großen Teil durch sein neuronales Netzwerk gesteuert wird [...]. Dies hat Konsequenzen sowohl für die philosophische Erkenntnistheorie, die nicht länger davon ausgehen [kann], dass das Subjekt unmittelbare Erfahrungen machen kann, die dann bewusst verarbeitet werden. [...] Dies hat nicht zuletzt Konsequenzen für das Alltagshandeln, wenn etwa die Frage nach der (juristischen oder moralischen) Schuldzuschreibung neu verhandelt werden muss." (Beer, R. / Grundmann, M. (2004), S. 3).

${ }^{196}$ Nollmann, G. (2006), S. 63.
} 
Vielmehr bin ich ihnen unterworfen."197 Hieraus schlussfolgert schließlich WILS: „Gerhard Roth plädiert für eine Abschaffung des alteuropäischen Subjekts von Verantwortung. “198 Insgesamt muss jedoch festgestellt werden, dass die Interpretationen der empirischen, neurowissenschaftlichen Ergebnisse hinsichtlich der Frage einer möglichen Willensfreiheit nach wie vor stark umstritten sind. ${ }^{199}$ Dennoch zeigt auch die neurowissenschaftliche Forschung mögliche Grenzen einer unkritischen, individuellen Verantwortungszuschreibung über den aufklärerischen Subjektbegriff auf.

Fasst man den heutigen Diskursstand zusammen, so lässt sich insgesamt ein intensiver Widerstreit hinsichtlich der Relevanz des aufklärerischen Subjektbegriffs feststellen ${ }^{200}$, wobei Formulierungen wie der ,Tod“ oder das „Verschwinden“ des Subjekts nahelegen mögen, dass einige Autoren die Position vertreten, diesen schillernden Begriff gar vollständig aufzugeben ${ }^{201}$, woraus sich wiederum eine teils überaus scharf geführte Kontroverse entwickelt hat. In diesem Sinne stellt auch STURMA fest, dass ,Vorbehalte gegenüber dem Subjektgedanken [.] mittlerweile überaus polemisch zum Ausdruck gebracht [werden]. “202 BURGER

${ }^{197}$ Roth, G. (1998), S. 330, in der Quelle ebenfalls herv. Vgl. auch Roth, G. / Strüber, N. (2019), S. 275. Diese Formulierung korrespondiert dabei erkennbarerweise mit der postmodernen Umdeutung des Subjektbegriffs zu etwas Unterworfenem. Vgl. auch die Überlegungen bei LEDOUX, welcher provokant konstatiert: ,You are your synapses. They are who you are." (LeDoux, J. E. (2003), S. 32). Vgl. hierzu jedoch auch kritisch Roth, G. / Strüber, N. (2019), S. 454.

198 Wils, J.-P. (1997), S. 28.

${ }^{199}$ Dabei stellt sich auch die Frage, wieso nun die Biologie wieder auf das Determinismusparadigma der klassischen Mechanik zurückfällt, welches die moderne Physik doch gerade überwunden hat. So stellt auch DÚsING fest, dass der strenge Kausaldeterminismus zur klassischen Physik gehöre, welcher in der modernen Physik nicht mehr vertreten werde, denn ,[d]er strenge Determinismus [...] gehört wissenschaftsgeschichtlich der früheren neuzeitlichen Physik an.“ (Düsing, K. (2005), S. 178). Vgl. hierzu auch die Überlegungen bei FALKENBURG, welche pointiert konstatiert: „Es ist erstaunlich, wie wenig die Hirnforscher, die den neuronalen Determinismus verkünden, diese physikalischen Grundlagen in Betracht ziehen.“(Falkenburg, B. (2012), S. 400). In diesem Kontext stellt PAUEN schließlich fest: „Es ist keineswegs sicher, ob insbesondere die Prozesse im Gehirn vollständig deterministischen Naturgesetzen unterliegen.“ Hierdurch bestehe auch „,kein akuter Grund zu der Annahme, Physik und Neurobiologie würden eines Tages zeigen, daß Freiheit gar nicht existiert, daß wir also nicht mehr sind als etwas kompliziertere Automaten, die für ihr Handeln nicht zur Rechenschaft gezogen werden können.“ (Pauen, M. (2001), S. 23-24).

200 Vgl. Bürger, P. (2001), S. 15.

${ }^{201}$ So postuliert auch KODALLE: „Die Überwindung einer subjekt-zentrierten philosophischen Argumentation gilt heute als Selbstverständlichkeit.“ (Kodalle, K.-M. (1994), S. 1).

202 Sturma, D. (2008), S. 25. 
formuliert dagegen nachdenklich: „Es ist noch nicht allzu lange her, da schien die Rede vom Tod des Subjekts den Schlüssel zum Verständnis unserer Gegenwart zu enthalten. Doch bevor man sich noch ernsthaft daran gemacht hatte, den Bedeutungsgehalt der befremdenden Formel zu ergründen, verlor man das Interesse an ihr [...]. Das ist bedauerlich, denn das Subjekt ist nun einmal die zentrale Kategorie der Moderne, die Rede von seinem Tode könnte also sehr wohl Ausdruck des Bewußtseins sein, daß wir an einer Epochenschwelle stehen. ${ }^{\prime 203}$ Neben der teils scharfen Subjektkritik haben sich in der jüngeren Zeit aber auch zunehmend Autoren hinsichtlich des aufklärerischen Subjektbegriffs verteidigend positioniert. ${ }^{204}$ So habe nach HEIDEMANN die kritische Auseinandersetzung gerade zur „Revitalisierung [...] des Subjekts“205 und nicht zu dessen Ende beigetragen. Auch BAUMGARTNER äußert sich ähnlich: „Die Rede vom Verschwinden des Subjekts in dieser allgemeinen Phrase ist ihrerseits totalisierend und als totalisierende Zeitdiagnose verfehlt. Es gibt in der Tat Begriffe von Subjektivität bzw. Aspekte des Subjektbegriffs, die verschwinden und verschwunden sind; darum dürfen aber nicht sogleich auch alle anderen Aspekte des Subjektbegriffs gleichermaßen für verschwunden erklärt werden. “206 Hieran anschließend subsumiert auch Dúsing: „Die geschilderten Phänomene des Selbstbewußtseins und der Selbstbeziehung von Personen dürften entgegen den Einwänden moderner Subjektkritiker schwerlich zu leugnen sein. Wache Menschen, denen solche Subjektivitätsphänomene intermittierend oder dauerhaft fehlen, befinden sich in der Regel, aus medizinischpsychologischer Perspektive, psychisch-geistig in einem defizitären oder kranken Zustand.“"207 Dies erscheint in der Tat einsichtig, betrachtet man wiederum das zuvor vorgestellte 3-Ebenen-Modell der Subjektivität, so dass in Anschluss an die

\footnotetext{
203 Bürger, P. (2001), S. 18.

${ }^{204}$ Als einer der bekanntesten Vertreter der Heidelberger Schule, welche sich gegen die Destruktion des Subjektbegriffs wendet, kann KLAUS DƯsING genannt werden. Vgl. hierzu die Ausführungen in Düsing, K. (2013), S. 7-8; Düsing, K. (2012), S. 159-160; Düsing, K. (2005), S. 129-134; Düsing, K. (1997), S. 9-17. Auch Fetz, HagenbUchle und SchulZ betonen: „Das Subjekt hat sein Ende überlebt. Die Subjektivität bestimmt weiterhin das Gespräch in Literatur und Philosophie, in den Geistes- und Gesellschaftswissenschaften. Selbst in systemtheoretischen Ansätzen bleibt sie erklärungsbedürftig. " (Fetz, R. L. / Hagenbüchle, R. / Schulz, P. (1998), S. V). Darüber hinaus findet sich z. B. bei ZIMA nicht nur eine Anwendung des Subjektbegriffs auf Kollektive, sondern sogar in Referenz auf Maschinen (vgl. Zima, P. V. (2017), S. 15-17). Vgl. ähnlich auch die Diskussion im Kontext von Algorithmen bei Reichertz, J. (2014), S. 111-113.

${ }^{205}$ Heidemann, D. H. (2002), S. 11, vgl. auch Bürger, P. (2001), S. 15; Hagenbüchle, R. (1998), S. 18-19.

206 Baumgartner, H. M. (1994), S. 27.

${ }^{207}$ Düsing, K. (2005), S. 132-133, vgl. auch Düsing, K. (1997), S. 123-128.
} 
vorigen Autoren in der Tat eine genauere Darlegung des spezifischen Begriffsverständnisses, welches in der neueren philosophischen Subjektkritik teils relativ unklar bleibt, anzumahnen wäre. ${ }^{208}$ Auch MUTSCHLER bezweifelt ein Verschwinden des Subjekts: „Mir hingegen scheint das Verschwinden des Subjekts kein kontingentes Faktum, jedenfalls dann nicht, wenn man an den Leistungen der Moderne wie Industrie, Technik oder Naturwissenschaft festhält. [...] Sobald man [.] die Leistungen der Moderne in Anspruch nimmt, steht man unaufhebbar unter dem Anspruch von Subjektivität, deren Verschwinden meines Erachtens bloßer Schein ist [...]. ${ }^{\text {209 }}$ Fraglich bleibt bei einer Aufgabe des aufklärerischen Subjektgedankens zudem, wie dann noch individuelle Verantwortung zu begründen wäre und ob hier nicht folglich der (Individual-)Ethik das zentrale argumentative Fundament entzogen würde. Denn, wie auch BAYERTZ feststellt, ,sind neben [.] empirischen Bedingungen auch bestimmte philosophische Annahmen, darunter vor allem die Idee der menschlichen Freiheit und Autonomie [relevant]. Mit dem Verzicht auf diese Annahmen wird die Idee der Verantwortung nicht weiterentwickelt, sondern verabschiedet." ${ }^{\text {210 }}$ Diese letztlich subjektphilosophische, individualethische Frage soll im Rahmen dieser Arbeit allerdings nicht geklärt werden, insbesondere auch, da diese auf die Mesoebene der Wirtschaftsethik fokussiert. Die vorausgegangenen Ausführungen mögen jedoch verdeutlichen, dass bereits im philosophischen Diskurs eine langanhaltende, starke Kontroverse um die Begriffsdeutung des Subjekts sowie dessen Relevanz an sich besteht. Neben diesen grundlegenden Überlegungen zeigen sich gerade auch für den wirtschaftsethischen Kontext vor dem Hintergrund der Globalisierung und

\footnotetext{
208 So kritisiert auch DUSING: ,Jedesmal wird eine selbständige Bedeutung schon eines empirischen Selbst oder Selbstbewußtseins, erst recht diejenige eines apriorischen Selbstbewußtseins geleugnet, und zwar ohne daß eine fundierte Auseinandersetzung mit einer der klassischen Subjektivitätstheorien insbesondere aus der Epoche von Kant bis Hegel stattfindet.“ (Düsing, K. (2013), S. 7). Vgl. auch die Überlegungen bei STURMA, welcher zur Sachlichkeit mahnt und die lange Historie und Komplexität des Subjektbegriffs betont: „Das Für und Wider des Subjektbegriffs hat vielmehr aus guten Gründen eine verschlungene und komplexe Geschichte. Wer es unterläßt, sie in ihren systematischen Konsequenzen zu bedenken, bewegt sich nicht mehr auf sachlichem Boden und kann schwerlich einen Beitrag zur Lösung der in der Tat noch nicht bewältigten Subjektproblematik leisten." (Sturma, D. (2008), S. 25). ${ }^{209}$ Mutschler, H.-D. (1994), S. 191.

${ }^{210}$ Bayertz, K. (1995), S. 5. Ebenso stellt auch HAGENBUCHLE fest: „Die gegenwärtige Infragestellung des Subjekts ist schon deshalb von Brisanz, weil die gesamte abendländische Kultur auf das selbstbestimmte Individuum hin zentriert erscheint. [...] Das Subjekt zu streichen, hieße den mündigen Menschen mit der ihm eigenen Freiheit und sozialen Verantwortlichkeit zu streichen." (Hagenbüchle, R. (1998), S. 2 und S. 16).
} 
Digitalisierung einige Implikationen hinsichtlich der Verwendung des aufklärerischen Subjektbegriffs zur Zuschreibung individueller Verantwortung. Dies ist Gegenstand der nachfolgenden Ausführungen. ${ }^{211}$

\subsubsection{Ethische Implikationen der globalisierten Wissensgesellschaft}

Hinsichtlich der Verantwortungszuschreibung lassen sich nun auch im wirtschaftsethischen Diskurs einige weitere Argumente auffinden, welche gerade auch für diesen fachspezifischen Kontext eine subjektbasierte, individuelle Verantwortungszuschreibung, besonders im Kontext der heutigen globalisierten Wissensgesellschaft, zunehmend problematisch erscheinen lassen. Die Beachtung der hiermit einhergehenden Transformationsprozesse ist neben den aufgezeigten grundlegenden philosophischen Überlegungen bedeutsam, bedenkt man die immer stärkere Einflussnahme globalisierter Wertschöpfungsketten auf individuelle wie kollektive Entscheidungen sowie die tiefgreifenden Veränderungen, welche die Digitale Transformation im Rahmen der heutigen Wissensgesellschaft hervorruft.

Ein erstes Argument, welches insbesondere durch die Globalisierung zunehmendes Gewicht erhält und zur Problematisierung einer rein individualsubjektiven Verantwortungszuschreibung herangezogen werden kann, stammt aus der makroethischen Schule der Wirtschaftsethik um HOMANN. Wie zuvor bereits diskutiert, ist diese Theorietradition durch eine Analyse moralischer Dilemmasituationen des Wirtschaftskontextes unter Anwendung des spieltheoretischen Instrumentariums geprägt $^{212}$, wobei HOMANN zum Schluss kommt, dass ein reiner Gebrauch von Moralappellen unter den Bedingungen der modernen Wirtschaft kritisch zu hinterfragen sei. So seien Individuen unter den Marktbedingungen einer globalisierten Weltwirtschaft vielfach überfordert, alleine den an sie gestellten Moralappellen zu folgen, welche diese dauerhaft (möglicherweise gar: existenziell) schlechterstellen würden. ${ }^{213}$ In diesem Sinne wäre, wie aufgezeigt wurde, ein reiner Moralappell nicht nur empirisch vielfach unbrauchbar bzw. faktisch unwirksam, um

${ }^{211}$ Diese beruhen grundlegend auf Lingnau, V. / Fuchs, F. (2019).

${ }^{212}$ Vgl. Lütge, C. / Uhl, M. (2017), S. 91-94; Suchanek, A. (2015b), S. 59-63; Homann, K. (2014), S. 72-75; Homann, K. / Lütge, C. (2013), S. 24-27; Suchanek, A. (2007), S. 53-57; Homann, K. / Suchanek, A. (2005), S. 35-38; Homann, K. (2002a), S. 95-97; Homann, K. / Blome-Drees, F. (1992), S. 29-32; Homann, K. (1991), S. 32-33.

${ }^{213}$ Dabei besteht nach HoMANN unter den Bedingungen der modernen Wirtschaft das Problem darin, , daß die aus moralischen Gründen erwünschten Ergebnisse nicht vom Handeln des einzelnen abhängen, sondern von den Handlungen zahlloser einzelner, die über einen unpersönlichen Mechanismus koordiniert werden.“ (Homann, K. (1991), S. 36). 
eigentlich wohlbegründete Ziele zu erreichen, sondern der reine Moralappell ohne Abwägung der individuell induzierten, korrespondierenden Konsequenzen sei auch ethisch kaum zu begründen und impliziere letztlich sogar die Gefahr einer Delegitimation ethischer Überlegungen selbst. ${ }^{214}$ Auf diesen Erkenntnissen aufbauend plädieren HOMANN und seine Schüler für eine institutionelle Entlastung der Individuen, vor allem auf der Makroebene der Wirtschaftsordnung, indem für alle Akteure gleiche, bindende Regeln geschaffen werden, so dass sich das normativ begründete Handeln zumindest nicht mehr dauerhaft nachteilig auswirke. ${ }^{215}$ Betrachtet man das Argument HomanNs damit aus subjektphilosophischer Perspektive, so befasst sich dieses mit der Realisierungsmöglichkeit des individualsubjektiven Handlungspotentials, welches im Rahmen einer globalisierten Wirtschaftsordnung kritisch zu hinterfragen wäre.

Ein weiteres Argument, welches im Rahmen klassischer Subjekteigenschaften hinsichtlich einer individuellen Verantwortungszuschreibung kritisch zu betrachten wäre, stammt aus dem Bereich der Technikethik, welche die arbeitsteiligen Verhältnisse der heutigen Wertschöpfungstätigkeiten in Wertschöpfungsketten bzw. -netzwerken ${ }^{216}$ in den Fokus rückt. So wird unter den Bedingungen der modernen Produktionsweise deutlich, dass eine relativ genaue, monokausale Verantwortungszuschreibung für das Endprodukt, welche in Manufakturbetrieben noch vielfach unproblematisch erschien, im Rahmen arbeitsteiliger Wertschöpfungsprozesse nahezu unmöglich ist; und dies gilt dann auch für eine individualethische Verantwortungszuschreibung. Wer letztlich für ein Ergebnis der Wertschöpfungstätigkeit innerhalb eines Unternehmens oder gar eines Wertschöpfungsnetzwerkes verantwortlich ist, lässt sich durch die vielfach interdependenten Entscheidungen kaum noch ermitteln. In diesem Sinne gilt mit DYBEL und SANDKUHLER: „Individuen sehen sich unkontrollierbaren anonymen Prozessen

${ }^{214}$ Vgl. Homann, K. (2015), S. 34; Homann, K. (2014), S. 64; Homann, K. / Lütge, C. (2013), S. 43; Homann, K. (2002f), S. 8-15.

${ }^{215}$ Vgl. Homann, K. / Lütge, C. (2013), S. 77; Homann, K. / Blome-Drees, F. (1992), S. 35. Vgl. grundlegend auch Werner, M. H. (2011a), S. 546. Wie bereits in Abschnitt 3.6 gezeigt, wird die ökonomisch begründete Position HoMANNS in der wirtschaftsethischen Literatur durchaus kontrovers diskutiert. Der Kerngedanke einer institutionellen Unterstützung verantwortungsbewussten Handelns hat jedoch auch eine positive Rezeption gefunden, so z. B. bei ULRICH, welcher ebenfalls die Bedeutung, institutioneller Rückenstützen“ hervorhebt. Vgl. Ulrich, P. (2010), S. 96-98.

${ }^{216}$ Vgl. einführend Becker, W. / Burggraf, A. / Martens, M. (2019), S. 169-173; Bach, N. et al. (2012), S. 102-106; Schuh, G. / Kampker, A. / Rittstieg, M. (2011), S. 477-479; Benger, A. (2007), S. 95-99; Blecker, T. / Liebhart, U. (2006), S. 1-4; Stengel, R. v. (1999), S. $18-20$. 
ausgeliefert und als Subjekte entmachtet.“217 Auch LENK hebt hervor: „Im Zeitalter der Großprojekte, der kollektiven Maßnahmen und Handlungen kann gegebenenfalls nicht der einzelne allein die Gesamtverantwortung für ein Gesamtunternehmen tragen, weil sein aktiver Beitrag bzw. seine Entscheidungsmacht äußerst begrenzt sind.“ ${ }^{218}$ Ebenso konstatiert BAUER: „Durch das Auslösen von Signalketten bewirkt ein Mensch Kaskaden von Effekten, für die er keine Verantwortung im eminenten Sinne übernehmen kann.“219 Dadurch werde schließlich auch ,die prinzipielle Unmöglichkeit von individueller Verantwortbarkeit sichtbar.“220 In diesem Zusammenhang droht, wie auch HUBIG feststellte, letztlich der Verlust des klassischen Handlungssubjekts. ${ }^{221}$ Insbesondere im Rahmen der Digitalen Transformation (,Industrie 4.0“) $)^{222}$ stellt sich die Frage nach der Verantwortungsübernahme für die Arbeitsergebnisse bzw. Tätigkeitskonsequenzen von Maschinen mit zunehmender Dringlichkeit. So ist bis heute umstritten, wer überhaupt und in welchem Umfang Verantwortung für Maschinentätigkeiten trägt: Konstrukteure, Softwareentwickler, das Aufsichtspersonal? Diese Problematik potenziert sich nochmals durch das Faktum, dass heute Entscheidungen in zunehmendem Maße nicht mehr individuell-autonom, sondern meist in Kollektiven (und zudem mit maschineller Unterstützung durchgeführt) getroffen werden. ${ }^{223}$

\footnotetext{
${ }^{217}$ Dybel, P. / Sandkühler, H. J. (2004), S. 7.

${ }^{218}$ Lenk, H. (1993), S. 125.

219 Bauer, C. A. (2018), S. 57.

${ }^{220}$ Bauer, C. A. (2018), S. 57.

${ }^{221}$ Vgl. Hubig, C. (1995), S. 61. In diesem Kontext wird in der Literatur auch vielfach das „problem of many hands“ diskutiert. So stellen bspw. VERMAAS ET AL. - insbesondere im systemischen Kontext - fest: „If undesired consequences arise in a sociotechnical system it is, in many cases, not possible to simply trace the cause of those consequences back to the actor who might have been able to foresee and prevent such consequences and who can, thus, be held responsible for them. It is much more likely that the consequences will depend on the actions of a number of actors and on the constellation of the sociotechnical system as a whole." (Vermaas, P. et al. (2011), S. 107). Vgl. hierzu einführend auch Poel, I. v. d. (2015b), S. 50-55; Peltonen, H. (2013), S. 46-47; Bovens, M. A. P. (1998), S. 45-50; Kaptein, M. (1998), S. 92-94 sowie klassisch Thompson, D. F. (2005), S. 11-12.

${ }^{222}$ Vgl. hierzu grundlegend Deckert, R. (2019), S. 1-6; Obermaier, R. (2019), S. 3-15; Steven, M. (2019), S. 13-23; Huber, W. (2018), S. 13-16; Ittermann, P. / Niehaus, J. (2018), S. 35-37; Lingnau, V. / Brenning, M. (2018), S. 138-141; Hänisch, T. (2017), S. 9-16; Lingnau, V. / Brenning, M. (2015), S. 455-456.

${ }^{223}$ Vgl. Stahl, B. C. (2000), S. 229.
} 
Neben diesen grundlegenden wirtschafts- und technikethischen Überlegungen existieren auch hinsichtlich der gesellschaftlichen Transformation zur Wissensgesellschaft Argumente, welche eine rein individualsubjektive Verantwortungszuschreibung zunehmend problematisch erscheinen lassen. So werden neuerdings auch verstärkt die Konsequenzen der Digitalisierung sowie die gesellschaftlichen Veränderungen durch die Wissensgesellschaft intensiv diskutiert. Der Begriff der Digitalisierung wird dabei in der Literatur in einer Vielzahl an Kontexten und inhaltlichen Deutungen gebraucht. ${ }^{224}$ Nimmt man allerdings eine Metaperspektive ein, so lassen sich im Kern zwei zentrale Begriffsverständnisse subsumieren. Im engeren Sinne referiert der Begriff lediglich auf eine Transformation analoger Objekte in binäre, d. h. digitalisierte Informationseinheiten. ${ }^{25}$ Dagegen bezeichnet der Begriff in einem weiteren Sinne die umfassende Durchdringung der gesamten menschlichen Lebenswelt mittels informatorischer Technologien, welche wiederum mit weitreichenden gesellschaftlichen Veränderungsprozessen korrespondieren. ${ }^{226}$ Gerade letzteres Verständnis schließt wiederum an die konzeptionellen Überlegungen zur aufkommenden Wissensgesellschaft an, in welcher die Verfügbarkeit von Wissen selbst zum zentralen Produktions- wie Erfolgsfaktor für Unternehmen wie auch Individuen wird. ${ }^{227}$ Dieser enge begriffliche Zusammenhang wird auch von GUTOUNIG hervorgehoben: „Die vorherrschende Digitalisierung und Vernetzung aller Lebensbereiche bildet so etwas wie den Kern der Wissensgesellschaft [...].“228

Vor dem Hintergrund der im Rahmen der Wissensgesellschaft stattfindenden technologischen sowie gesellschaftlichen Veränderungen kommt dabei NEUSER zum Schluss, dass das moderne Individuum (zumindest hinsichtlich der freien Erkenntnisfähigkeit) nicht mehr als Subjekt im aufklärerischen Sinne aufgefasst werden kann: „Seine zentrale Stellung, die das Subjekt in den subjekttheoretischen Konzepten als Erzeuger von Wissen hatte, ist verloren.“229 Damit

${ }^{224}$ Vgl. zum Überblick Becker, W. (2019), S. 24; Botzkowski, T. (2018), S. 22-24.

${ }^{225}$ Vgl. Spengler, T. / Metzger, O. / Volkmer, T. (2019), S. 247; Kröhling, A. (2017), S. 24; Mertens, P. / Barbian, D. / Baier, S. (2017), S. 35; Picot, A. / Hopf, S. / Sedlmeir, J. (2017), S. 88; Renninger, W. (2016), S. 74; Becker, J. (2013), S. 33.

${ }^{226}$ Vgl. Bohlen, W. (2019), S. 278; Breuing, K. (2019), S. 109-110; Hirsch-Kreinsen, H. (2018), S. 14; Picot, A. / Hopf, S. / Sedlmeir, J. (2017), S. 88-89; Renninger, W. (2016), S. 74-75.

${ }^{227}$ Vgl. Wohlwender, A. (2015), S. 17-18; Haunss, S. (2013), S. 58; Drucker, P. F. (2012), S. 164-165; Probst, G. / Raub, S. / Romhardt, K. (2012), S. 3; Rolff, H.-G. (2010), S. 179; Weinreich, J. (2009), S. 167; Prescher, C. / Eisfeldt, D. (2006), S. 405-406.

228 Gutounig, R. (2015), S. 95.

${ }^{229}$ Neuser, W. (2013), S. 50. 
wird insbesondere der seit DESCARTES eingeführte epistemologische Aspekt des Subjektbegriffs als zentrale Grundlage der Sicherheit für die Erkenntnis eines Individuums in Zweifel gezogen, welches folglich Konsequenzen für die darauf aufbauenden Handlungen impliziert. In der Tat scheint im Rahmen der Digitalisierung dieses aufklärerisch geprägte Fundament zu erodieren, da in zunehmendem Maße informationstechnische Systeme bei der Generierung von Wissen maßgeblich und relativ autonom involviert sind. So sind beispielsweise die Erzeugung komplexer Wissensbestände wie auch anspruchsvolle Konstruktionen ohne technische Unterstützung kaum mehr möglich. In diesem Sinne betont auch NeUgebaUER: „Ein Prozessor mit Transistoren in Milliardenzahl etwa ist nur mit weitgehend automatisierten, digitalisierten Verfahren zu konstruieren und herzustellen. Komplexe Programme wiederum werden ganz oder teilweise von Rechnern selbst entworfen, umgesetzt und geprüft. [...] Künstliche Systeme sammeln Erfahrungen und können diese anschließend verallgemeinern. Sie erzeugen Wissen.“230 Dadurch wiederum, wie NeUSER feststellt, „bilden sich Wissenssysteme aus, die nicht mehr subjektgesteuert sind. Dies gilt auch, wenn sie durch Individuen, durch Ingenieure angetriggert werden." 231 Diese technologische Transformation wirft nun wiederum eine Reihe von ethischen Fragen auf, so z. B. ,über neue Formen von Deutung von Welt nachzudenken, die nicht mehr von einem Subjekt verantwortet sind [...].“232 In jedem Fall zeigt damit auch die Diskussion im Kontext der Digitalisierung, dass der Status als individuelles, freies Subjekt mit dem Primat der Letztverantwortung zumindest überaus kritisch rezipiert wird.

Die vorausgegangenen Überlegungen haben im Kontext der Digitalen Transformation aber auch Implikationen auf den zentralen Begriff des Wissens selbst, weshalb auf diesen abschließend kurz eingegangen sei. Grundsätzlich ist Wissen nach BECKER ein „traditionsreicher und schillernder Begriff“"233 und, wie auch WEINREICH hervorhebt, ,in der Geschichte der Menschheit schon immer von Bedeutung gewesen. “234 Neben der alltagssprachlichen Bedeutung wird der Begriff aber auch in diversen Fachdisziplinen gebraucht. Dabei lassen sich im

\footnotetext{
${ }^{230}$ Neugebauer, R. (2018), S. 1-2.

${ }^{231}$ Neuser, W. (2014), S. 7, vgl. auch Neuser, W. (2017), S. 69. Vgl. zudem die Überlegungen von ORNELLA, welcher den Subjektbegriff weiterhin beibehält, allerdings feststellt, dass heute das ,Subjekt als vernetztes und medialisiertes verstanden werden [müsse].“ (Ornella, A. D. (2010), S. 158).

${ }^{232}$ Neuser, W. (2014), S. 1.

233 Becker, E. (2016), S. 169. Vgl. auch Meynhardt, T. (2004), S. 47.

${ }^{234}$ Weinreich, C. (2010), S. 15.
} 
Kern zwei verbreitete Konzeptionen differenzieren, welche zum besseren Verständnis kurz dargestellt und voneinander abgegrenzt werden. In einem ersten Schritt wird dabei aus philosophischer Perspektive häufig mit Referenz auf PLATON Wissen als wahre, gerechtfertigte Meinung definiert. ${ }^{235}$ Wissen weist in diesem Sinne folglich drei konstitutive Merkmale auf: So muss erstens eine Meinung vorliegen, d. h. ein Individuum (bzw. ein Subjekt!) muss von einem Sachverhalt selbst überzeugt sein. Darüber hinaus müssen jedoch auch gute Gründe $^{236}$ für die existierende Meinung vorliegen, d. h., diese darf nicht willkürlich oder zufällig zustande gekommen sein. Schließlich ist drittens zu fordern, dass die Meinung nicht nur gerechtfertigt, sondern auch wahr ist, welches bspw. im korrespondenztheoretischen Sinne ${ }^{237}$ die Übereinstimmung mit der Realität bzw. Wirklichkeit ${ }^{238}$ impliziert. $^{239}$

${ }^{235}$ Vgl. Platon (1856), 201c-201e, welcher jedoch eigentlich auch diese Definition als unzureichend erachtet und verwirft. Vgl. hierzu auch kritisch die Anmerkungen bei Gabriel, M. (2016), S. 37 und S. 48 sowie Gabriel, M. (2014), S. 149, Fn. 128 Kapitel 2.

236 Vgl. hierzu Hoffmann, M. (2008), S. 235; Forst, R. (2007), S. 41; Gottschalk-Mazouz, N. (2004), S. 175-178; Nida-Rümelin, J. (2001), S. 74-82; Gosepath, S. (1999), S. 8 sowie die Überlegungen in Abschnitt 3.1.2.

${ }^{237}$ Diese Konzeption findet sich schon bei Aristoteles (2009), 1051b. Wahre Aussagen sind hierbei durch die Übereinstimmung mit der (bzw. einer angenommenen) Realität gekennzeichnet (vgl. z. B. Grundmann, T. (2017), S. 32-33; Gabriel, M. (2016), S. 182; Baumann, P. (2015), S. 155; Kobusch, T. (2006), S. 149; Janich, P. (2005), S. 31). Diesem klassischen Begriffsverständnis wird häufig kontrastierend die Kohärenztheorie gegenübergestellt. Vgl. z. B. Schulz, G. (1993), S. 5. Vgl. auch Brühl, R. (2017), S. 31-36; Baumann, P. (2015), S. 155-178, welche zudem die Konsenstheorie der Wahrheit als dritten möglichen Zugang herausstellen (vgl. hierzu auch klassisch Habermas, J. (1984), S. 136-137) sowie Pap, A. (1955), S. 57-58, welcher drittens einen pragmatischen Zugang zum Wahrheitsbegriff unterscheidet. Vgl. zu letzterem auch die Ausführungen in Spohn, W. (2013), S. 180. Vgl. auch die detaillierten Ausführungen bei Gloy, K. (2004), S. 67-76.

238 Beide Begriffe werden in der Literatur häufig mehr oder minder synonym gebraucht (so z. B. auch bei KANT, vgl. Schöndorf, H. (2010b), S. 390). Vgl. im Kontext der Wirtschaftswissenschaften auch Helfrich, H. (2016), S. 80-81; Kolb, G. (2012), S. 4. Teilweise wird allerdings, gerade in der deutschsprachigen Philosophie, auch der Versuch unternommen, diese Begriffe genauer zu differenzieren. Eine verbreitete Auffassung unterscheidet hierbei zwischen der Realität (lat. realitas), welche etwas Dingliches (lat. res) beschreibt, während Wirklichkeit etwas Wirkendes, Aktives, vielfach aber auch Subjektives repräsentiert. Der Begriff der Wirklichkeit (lat. actualitas) wurde dabei von dem mittelalterlichen Philosophen MEISTER ECKHART geprägt. Vgl. hierzu Kirchner, F. et al. (2013), S. 551-552 und S. 735736; Schöndorf, H. (2010b), S. 389-390; Schöndorf, H. (2010e), S. 580-581; Kistner, P. (2006), S. 114; Schmidt, H. / Schischkoff, G. (1982), S. 573-574 und S. 754-755.

${ }^{239}$ Vgl. Gabriel, M. (2016), S. 36; Brendel, E. (1999), S. 18-19. 
Obschon diese Auffassung, gerade im philosophischen Kontext, durchaus noch immer recht verbreitet ist, hat die Literatur im Laufe der Zeit auch einige Kritik an dieser Wissenskonzeption hervorgebracht, so z. B. im Rahmen des kontrovers diskutierten GeTTIER-Problems. ${ }^{240}$ Für die vorliegende Arbeit sei jedoch vor allem herausgestellt, dass bereits die erste Voraussetzung, d. h. die Existenz einer Meinung, ein denkendes, reflexions- und begründungsfähiges Wesen ${ }^{241}$ mithin ein Subjekt $t^{242}$ - voraussetzt, welches damit eine maschinelle Generierung von Wissen prinzipiell ausschlösse. Geht man jedoch gerade vor dem skizzierten Hintergrund der Wissensgesellschaft davon aus, dass in zunehmendem Maße auch technische Systeme relativ autonom Wissen erzeugen und austauschen können, so erscheint der platonische Wissensbegriff zu eng und ein anderer konzeptioneller Zugang notwendig. So stellt auch GLOY paradigmatisch fest: „Daß der neue Träger des Wissens, der Computer, und die ihm eigentümliche Sprache, nämlich die moderne Algebra und Information, zusammen mit der immensen Speicherkapazität des Wissens, ja die gesamte semiologische Wende einen ganz neuen Wissensbegriff generiert, ist nicht zu übersehen. “243 Ein solcher Begriff kann dabei in einem informationswissenschaftlichen Kontext aufgefunden werden, welcher gerade in jüngerer Zeit und insbesondere auch im betriebswirtschaftlichen Schrifttum steigende Verbreitung erfahren hat. ${ }^{244}$ Wissen kann dabei in einem begriffshierarchischen Sinne aufgefasst werden, wobei der Zusammenhang häufig anhand der s. g. Wissenspyramide visualisiert wird. So stehen auf der untersten Ebene die einzelnen Zeichen, welche mittels syntaktischer Regeln zu Daten aggregiert werden können. Hierauf aufbauend kann im Rahmen einer Semantik, d. h. durch Hinzufügen von Bedeutung, die reine Datenstruktur zur Ebene der Information überführt werden. Werden nun diese Informationen wiederum miteinander verknüpft bzw. in Verbindung zueinander gesetzt, so entsteht auf der letzten

${ }^{240}$ Vgl. Gettier, E. L. (1963), welcher argumentiert, dass wahre, gerechtfertigte Meinung nicht unter allen Umständen immer plausibel als Wissen gelten könne. Vgl. einführend auch Hetherington, S. (2019), S. 2-4; Hetherington, S. (2016), S. 1-9; Ernst, G. (2013), S. 69-78; Lemos, N. (2007), S. 22-27; Enskat, R. (2005), S. 99-102.

${ }^{241}$ Die Frage, inwiefern dieses Wissen bewusst repräsentiert sein muss, wird in der Literatur allerdings kontrovers diskutiert. Vgl. Meynhardt, T. (2004), S. 50.

${ }^{242}$ Vgl. z. B. Gabriel, M. (2016), S. 36, welcher auch explizit auf diesen Terminus rekurriert. Vgl. ähnlich auch Ernst, G. (2013), S. 77-78.

243 Gloy, K. (2005), S. 7.

${ }^{244}$ Vgl. z. B. Gust von Loh, S. (2009), S. 11; Müller, B. (2009), S. 26; Schröder, K. A. (2003), S. 15; Schomann, M. (2001), S. 19. Für einen Überblick über weitere Definitionen des Wissens im betriebswirtschaftlichen Kontext vgl. die Übersicht bei Bodrow, W. / Bergmann, P. (2003), S. 35-37. 
Stufe schließlich Wissen. ${ }^{245}$ Diese konzeptionellen Überlegungen verdeutlicht auch nochmals die nachfolgende Abbildung 4.3.

Abbildung 4.3 Der informationswissenschaftliche Wissensbegriff in hierarchischer Darstellung

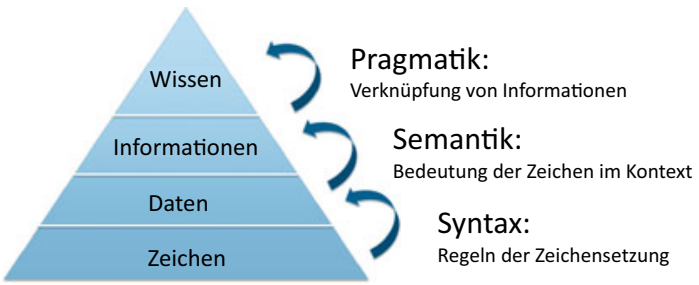

Dabei wird ersichtlich, dass im Rahmen einer solchen Konzeption der Besitz von Wissen nicht die Existenz eines Subjektes voraussetzt, sondern prinzipiell auf technische Einheiten Anwendung finden kann, welche z. B. mittels Big Data-Analysen Zusammenhänge zwischen Informationen herausarbeiten und dem Nutzer $^{246}$, aber auch anderen technischen Systemen, zur Verfügung stellen können. Damit erscheint dieser Begriff für die technologischen Veränderungen im Rahmen der Wissensgesellschaft fruchtbar und wird daher auch den weiteren Ausführungen dieser Arbeit zugrunde gelegt.

Fasst man die bisherigen Ausführungen zusammen, so wird deutlich, dass die Zuschreibung von individueller Verantwortung vor dem Hintergrund klassischer subjekttheoretischer Vorstellungen aus verschiedenen Argumentationslinien zunehmend problematisch erscheint. Während im philosophischen Diskurs teilweise eine vollständige Umdeutung des Subjektbegriffs oder gar die Elimination des aufklärerischen Subjektbegriffs intensiv diskutiert wurde, stellt sich insbesondere auch vor dem Hintergrund der Globalisierung und Digitalisierung zunehmend die Frage, inwiefern eine traditionelle, subjektbasierte, individuelle Verantwortungszuschreibung unter diesen Rahmenbedingungen noch zu überzeugen vermag. Dabei zeigten die vorigen Ausführungen, dass die traditionell unterstellte autonome Erkenntnisfähigkeit bzw. Wissensgenerierung durch die Digitale Transformation bedroht scheint, während sich durch komplexere,

245 Vgl. Engelmann, F. / Großmann, C. (2018), S. 5-6; Hutzschenreuter, T. (2015), S. 463; Halft, S. (2013), S. 10; Probst, G. / Raub, S. / Romhardt, K. (2012), S. 16; Stock, M. (2010), S. 11-12; Gust von Loh, S. (2009), S. 11-12; Müller, B. (2009), S. 26; Reucher, E. (2009), S. 23-24; Scherm, E. / Pietsch, G. (2007), S. 300-301; Bodendorf, F. (2006), S. 1-2; Bodrow, W. / Bergmann, P. (2003), S. 38.

${ }^{246}$ In diesem Zusammenhang differenziert z. B. GLOY „Erkenntnis“ bzw. „Einsicht“ von „Wissen“: „Wissen ist eine Ressource, auf die man jederzeit zurückgreifen kann, während Erkenntnis und Einsicht eher temporär sind." (Gloy, K. (2005), S. 10). 
global-interdependente Wertschöpfungstätigkeiten eine Begrenzung individueller Handlungsmöglichkeiten sowie eine Diffusion der Handlungskausalität ergibt. Als Zwischenfazit erscheint damit die Referenz auf das Individuum zumindest als primärer oder gar alleiniger Verantwortungsträger zunehmend fraglich. Eine solche „Leerstelle“ wäre auf den ersten Blick hochproblematisch, ist doch die abendländische Ethik durch einen klaren Fokus auf das Individuum als frei erkennende, handelnde und damit verantwortliche Entität gekennzeichnet. ${ }^{247}$ Inwiefern jedoch Individuen tatsächlich in der Lebenswirklichkeit moderner, komplexer Gesellschaften noch als primäre Adressaten der Verantwortungsübernahme aufzufassen sind, scheint vor dem aufgezeigten Hintergrund allerdings mehr als kritisch zu sehen. So wird auch die individuelle Verantwortung im öffentlichen Diskurs zwar „zunehmend gefordert, aber auch zunehmend als Überforderung abgewehrt.“248 Im Transfer auf die Mesoebene kann mit ROPOHL folglich konstatiert werden: „Dem Risiko im Prinzip Verantwortung ist die individualethische Moralphilosophie nicht gewachsen. [...] Gleichwohl muß das Prinzip Verantwortung, wenn es sich nicht in praxisfremden Appellen erschöpfen soll, die individualethische Begrenzung überwinden [...].“249 Ebenso stellt auch WIELAND fest: „Ethik und Moral der modernen Wirtschaft des 21. Jahrhunderts aber wenden sich nicht mehr alleine an das Wirtschaftsindividuum, sondern direkt und immer häufiger in

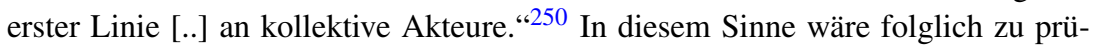
fen, inwiefern die entstandene „Verantwortungslücke“ nun mehr durch kollektive

${ }^{247}$ Vgl. Maring, M. (2001b), S. 107. In diesem Sinne stellt auch KAUFMANN fest: „Das Individuum - und nur noch das Individuum - gilt als moralisches Subjekt [...].“ (Kaufmann, F.-X. (1995), S. 80). Ähnlich betont auch LOH, welche nochmals die enge Verbindung zum Begriff der Person hervorhebt: „Innerhalb des Verantwortungsdiskurses ist man sich zumindest darüber einig, dass Verantwortung traditionell ein individualistisches Prinzip darstellt [...].“ (Loh, J. (2017), S. 40, vgl. ähnlich auch Fetzer, J. (2004), S. 123). Wie bereits in Abschnitt 3.5 gezeigt wurde, ist gerade auch in der angelsächsischen Literatur häufig ein sehr starker Fokus auf das Individuum als letztverantwortliche Einheit festzustellen (vgl. z. B. Arnold, D. G. / Beauchamp, T. L. / Bowie, N. E. (2020), S. 1; Ferrell, O. C. / Fraedrich, J. / Ferrell, L. (2017), S. 2-3; Velasquez, M. G. (2014), S. 56-57). Diese Grundhaltung fassen auch ZIMMERLI und AßLANDER wie folgt zusammen: ,[T]he subject of responsibility can usually only be a single individual [...].“ (Zimmerli, W. C. / Aßländer, M. S. (2010), S. 43). Ähnlich subsumiert auch BOHRMANN diese Auffassung, dass ,,nur der Mensch als vernunftbegabtes (rationales) und freies (autonomes) Wesen [.] in der Lage [sei], für das, was er tut oder unterlässt, zur Rechenschaft gezogen zu werden und folglich dafür auch einzustehen." (Bohrmann, T. (2018), S. 28).

248 Birnbacher, D. (1995), S. 143.

${ }^{249}$ Ropohl, G. (1994), S. 118.

${ }^{250}$ Wieland, J. (2005b), S. 11. Ähnlich resümieren auch LENK und MARING: „In der abendländischen Tradition beziehen sich Typen ethischer Begründung sowie universalmoralische 
bzw. korporative Akteure ${ }^{251}$ zu schließen wäre, und inwiefern der aufklärerische Subjektbegriff in diesem Kontext dann wieder zur Begründung von organisationaler Verantwortung herangezogen werden könnte. Dies ist Gegenstand der nachfolgenden Ausführungen. ${ }^{252}$

\subsubsection{Kollektive Akteure als alternative Verantwortungsträger?}

Folgt man den vorigen Überlegungen, so stellt sich die Frage, inwiefern möglicherweise gerade kollektive Einheiten die aufgezeigten Schwächen der Verantwortungsübernahme zumindest teilweise kompensieren könnten, indem sie die Komplexität moderner Gesellschaften wieder handhabbarer werden lassen und ggf. anstelle oder zumindest zusätzlich zu den Individuen als (kollektives) moralisches Verantwortungssubjekt aufgefasst werden könnten. So hebt auch STAHL hervor: „Das Individuum hat weder das nötige Wissen bezüglich der Resultate seiner Handlungen noch die Macht, diese zu beeinflussen. Gleichzeitig, und vielleicht auch deswegen, werden Institutionen, Organisationen, Korporationen etc. zu den relevanten Akteure [sic!] der Gesellschaft““253, denn es gilt, dass „Kollektive mehr Macht haben als Individuen, dass sie die nötigen Ressourcen haben, um mit Komplexität umzugehen und dass sie eher in der Lage sind, Erkenntnisse in die Zukunft zu projizieren." ${ }^{\text {254 }}$

Regeln nahezu ausschließlich auf das Handeln und Leben von Individuen. In unserer Industriegesellschaft treten demgegenüber heutzutage in starkem Ausmaß und zunehmend auch Phänomene des kollektiven Handelns [...] sowie andere verwandte Probleme auf, die mit einer streng individualistischen Universalmoral nicht mehr erfaßt werden können." (Lenk, H. / Maring, M. (1996), S. 7).

${ }^{251}$ Autoren wie NEUHÄUSER oder auch MARING unterscheiden an dieser Stelle teils noch explizit zwischen kollektiven und korporativen Akteuren, wobei korporative Akteure dann als Teilmenge der kollektiven Akteure zu verstehen sind und durch ihre Zeitstabilität und gemeinsame Handlungsstruktur gekennzeichnet sind. Vgl. Neuhäuser, C. (2011a), S. 151; Maring, M. (2001b), S. 108-110. Da im Folgenden bei den sozialen Subsystemen in Unternehmen stets von solchen zeitstabilen, koordiniert handlungsfähigen Systemen ausgegangen wird (vgl. Abschnitt 4.5.3), seien die beiden Begriffe im Rahmen dieser Arbeit relativ synonym gebraucht. Alle kollektiv planvoll handlungsfähige soziale Einheiten sind demnach „korporative Akteure“ im obigen Begriffsverständnis.

${ }^{252}$ Die nachfolgenden Ausführungen beruhen grundlegend auf Lingnau, V. / Fuchs, F. (2019).

253 Stahl, B. C. (2000), S. 228.

${ }^{254}$ Stahl, B. C. (2000), S. 229. 
Auch FetZer betont die zunehmende Bedeutung ,gesellschaftlicher Kausalitätszentren, die sehr wohl etwas ,tun', deren Taten jedoch Individuen nicht zugerechnet werden können - und zwar nicht nur im Einzelfall nicht, sondern systematisch [...].“255 Dabei kann jedoch nicht nur eine ledigliche Verantwortungsdelegation auf die Makroebene, d. h. zum Staat, stattfinden, denn gerade durch die Globalisierung haben die Einzelstaaten deutlich an Handlungsmacht eingebüßt, während große Konzerne deutlich an Gestaltungsmöglichkeiten hinzugewonnen haben. ${ }^{256}$ Folglich reicht ein Fokus auf die Gestaltung von Rahmenordnungen im Sinne klassischer Ordoethiken, wie im Rahmen der Konzeption HoMANNS diskutiert, nicht aus, womit zunehmend Verantwortung an Unternehmen zurückfällt, ihre Handlungen überzeugend zu begründen. Die Frage ist jedoch, wie diese Verantwortungspflicht selbst zu begründen wäre. Eine Überlegung könnte dabei sein, auf das traditionelle Begründungsmuster des moralfähigen Subjektes (z. B. im Sinne eines ehrbaren Kaufmanns auf der Mesoebene) zu rekurrieren, wodurch eine Zuschreibung von Verantwortung auch auf der kollektiven Ebene möglich wäre. In diesem Sinne betont auch BOHRMANN: „Als Subjekt gilt die freie Person, die sich ihrer Handlungen bewusst ist; in der ethischen Tradition wird sie als vernunftbegabt, moral- und zurechnungsfähig verstanden. Allerdings sind nicht nur Individuen damit gemeint, sondern Träger von Verantwortung können ebenso eine Organisation, eine Gruppe, ein Staat, ein Unternehmen, eine Religionsgemeinschaft sein [...]." ${ }^{\text {257 }}$ Aus dieser Perspektive wäre es demnach bedeutsam, folgende Frage zu klären: „Können Unternehmen innerhalb der moralischen Kommunikation als Subjekte wahrgenommen und angesprochen werden? ${ }^{* 258}$

Inwiefern der Begriff des moralfähigen Subjektes bzw. der moralfähigen Person auch auf kollektiv handelnde Entitäten übertragen werden kann, wird in der Literatur überaus kontrovers diskutiert. Der bekannteste Vertreter der Auffassung, dass Korporationen als moralische Personen und damit als verantwortlich handelnde Einheiten aufgefasst werden können, ist PETER FRENCH. Das zentrale Argument FRENCHS lautet hierbei, dass durch die gegebene interne Entscheidungsstruktur (Corporate Internal Decision [CID] Structure) Entscheidungen immer durch Weisungs- und Zuständigkeitsbefugnisse vorgefiltert und dann durch die legitimierten Organe für den kollektiven Akteur getroffen werden, so dass diese der Korporation direkt intentional zugerechnet werden können:

\footnotetext{
255 Fetzer, J. (2004), S. 111.

${ }^{256}$ Vgl. Neuhäuser, C. (2011a), S. 14. Vgl. auch Wettstein, F. / Waddock, S. (2005), S. 304.

${ }^{257}$ Bohrmann, T. (2018), S. 27.

${ }^{258}$ Fetzer, J. (2004), S. 37.
} 
„A functioning CID Structure incorporates acts of biological persons. “259 Durch die zugeschriebene Fähigkeit, korporative Intentionen durch gemeinsame Entscheidungsprozeduren zu bilden, kommt FRENCH schließlich zu dem Schluss, dass Unternehmen als moralische Personen aufgefasst werden können, welche dadurch wiederum Handlungsverantwortung (aber auch bestimmte fundamentale Rechte!) besitzen: „In short, corporations can be full-fledged moral persons and have what-ever privileges, rights and duties are, in the normal course of affairs, accorded to moral persons. " 260 Obwohl diese Überlegungen durchaus in der wirtschaftsethischen Literatur verbreitet sind und umfassend rezipiert wur$\operatorname{den}^{261}$, hat sich doch bereits einige Jahre nach erstmaliger Veröffentlichung der Überlegungen FRENCHS auch deutlicher Widerstand gegen eine Auffassung von Unternehmen als moralfähigen Subjekten entwickelt. Die bekannteste Gegenposition stammt hierbei von VELASQUEZ, welcher sich explizit gegen FRENCHS Ausführungen positioniert. ${ }^{262}$ Er betont konträr zu dessen Konzeption, dass letztlich nur (oder zumindest primär) Individuen Verantwortungsträger repräsentierten und die Auffassung, dass ein Unternehmen "handele“ nur in einem elliptischen, d. h. verkürzenden, Sinne aufgefasst werden könne und für nicht bekannte, eigentlich handelnde Individuen stehe. ${ }^{263}$

Betrachtet man diese beiden Extrempositionen, so ist bis heute nicht geklärt, welcher dieser beiden Auffassungen nun der Vorzug gegeben werden könnte, wenngleich selbst VELASQUEZ darauf hinweist, dass im Laufe der Zeit tendenziell eher der Position FrENCHS gefolgt wurde. ${ }^{264}$ Das zentrale Problem hierbei erscheint der letztlich metaphysische Gehalt des aufklärerischen Subjektbegriffs zu sein ${ }^{265}$, welcher letztlich weder formal noch empirisch geprüft werden kann. Folglich existieren grundsätzlich auch weiterhin Fachvertreter, die

${ }^{259}$ French, P. A. (1979), S. 212, in der Quelle ebenfalls herv. In diesem Sinne stellt FRENCH auch an anderer Stelle heraus: „Statements ascribing responsibility to a conglomerate are not reducible to a conjunction of statements ascribing responsibility to the individuals associated with the conglomerate." (French, P. A. (1998), S. 45).

${ }^{260}$ French, P. A. (1979), S. 207.

${ }^{261}$ Vgl. z. B. Göbel, E. (2020), S. 110-115; Crane, A. et al. (2019), S. 46-48; Schöttl, L. (2018), S. 17-22; Heidbrink, L. (2017), S. 20-21; Shaw, W. H. (2017), S. 160-162; Aßländer, M. S. / Brink, A. (2008), S. 110-111; Fetzer, J. (2004), S. 103-116; Maring, M. (2001a), S. 251; Kleinfeld, A. (1998), S. 126-127; Danley, J. R. (1993), S. 279-282.

262 Vgl. Velasquez, M. G. (1983), S. 6-10.

${ }^{263}$ Vgl. Velasquez, M. G. (2014), S. 17-18; Velasquez, M. G. (2003), S. 543; Velasquez, M. G. (1983), S. 13.

264 Vgl. Velasquez, M. G. (2003), S. 531.

265 Vgl. auch French, P. A. (1986), S. 19. 
einen moralischen Personen- bzw. Subjektstatus auf Unternehmensebene verteidigen $^{266}$, aber auch kritische Stimmen, welche korporative Subjektzuschreibungen

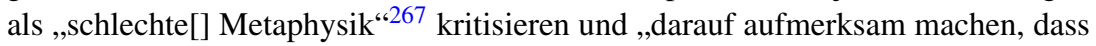
soziale Institutionen und Organisationen letztlich aus einzelnen Individuen bestehen, die alle für ihre jeweiligen Aufgabenbereiche verantwortlich sind [...].“268 Auch HÄRLE betont, dass ,ethische Subjekte [.] immer nur einzelne Menschen mit ihren Intentionen und ihrer ethischen Verantwortung [sind]." ${ }^{269}$ Genau dieser Standpunkt erscheint jedoch aus den im vorigen Kapitel aufgezeigten Gründen in der heutigen Zeit zunehmend problematisch. Darüber hinaus könnte an der Auffassung von VELASQUEZ kritisiert werden, dass dieser Korporationen nur als Summe von Individuen betrachtet ${ }^{270}$, wodurch erstens technische Elemente vernachlässigt werden und zweitens unbeachtet bleibt, dass ein System durch die existierenden Beziehungen (bei FRENCH: die CID) stets mehr ist, als die Summe seiner Teile. ${ }^{271}$ In diesem Sinne betont auch MARING: „Handlungssysteme ab der Mesoebene sind eben nicht eine bloße Menge von Individuen, sondern sozial verbundene, strukturierte Mengen. Diese Struktur wirkt auf die Individuen ein, ebenso wie diese die Struktur mitprägen.“272 Ähnlich hebt auch SOARES hervor

\footnotetext{
266 Vgl. z. B. die Überlegungen bei Dubbink, W. / Smith, J. (2011), S. 233-241; Arnold, D. G. (2006), S. 288-291; Silver, D. (2005), S. 279-280.

${ }^{267}$ Zimmerli, W. C. / Palazzo, G. (1998), S. 188.

268 Bohrmann, T. (2018), S. 33.

${ }^{269}$ Härle, W. (2018b), S. 92. Pointiert stellt auch REICHERTZ fest: „Es sind immer konkrete Menschen, die handeln.“ (Reichertz, J. (2010a), S. 22). Ähnlich postuliert BESCHORNER: „Nur Personen können ethisch handeln. Organisationen, z. B. Unternehmen, koordinieren immer ,nur ' die individuellen Handlungen von Personen." (Beschorner, T. (2010), S. 116). Eine weitere Überlegung stammt hierbei von WERHANE, die für Korporationen den Status als „,moral persons“ ablehnt und diese vielmehr als ,secondary actors“ auffasst, welche sich aus individuellem Handeln von Menschen für diese Korporationen ergeben (vgl. Werhane, P. H. (2016), S. 5-6 und S. 16-17; Werhane, P. H. / Freeman, R. E. (2005), S. 522-524; Werhane, P. H. (1989), S. 821-822; Werhane, P. H. (1985), S. 49-59). In diesem Zusammenhang stellt allerdings STAHL fest: „Dieses Argument unterscheidet sich bei genauerer Betrachtung nur in der Wortwahl von dem Frenchs. Ob man von einer moralischen Person spricht oder von einem sekundären Aktor, das Ergebnis bleibt gleich. Beide Argumentationen laufen darauf hinaus, Korporationen moralische Verantwortung zuzuschreiben ohne dabei zu behaupten, dass Kollektive mit natürlichen Personen identisch sind. Dennoch bilden die Kollektive in beiden Fällen wieder etwas, das über ihre Mitglieder hinausgeht, sei es die ,moralische Person“ oder den ,sekundären Aktor“." (Stahl, B. C. (2000), S. 231).

${ }^{270}$ Vgl. Velasquez, M. G. (1983), S. 1.

${ }^{271}$ Vgl. klassisch Aristoteles (2009), 1041b sowie im Folgenden die detaillierteren Ausführungen zur Systemtheorie in Abschnitt 4.3.

272 Maring, M. (2001b), S. 133.
} 
,[t]hat the notion of a corporation cannot be reduced to the mere behaviour of individuals [...].“273

Ein fruchtbarer Ansatz, welcher die beiden konfligierenden Positionen teilweise verbindet, könnte dabei in der Position NeuHÄUSERS gefunden werden, welcher argumentiert, dass Unternehmen nicht als moralische Personen aufgefasst werden sollten, da diesen bestimmte traditionell mit Personen assoziierte Rechte, wie z. B. die zentralen Menschenrechte, nicht zuerkannt werden können: „Personen haben eine Würde, Unternehmen hingegen nicht. Daher sind Unternehmen keine Personen.“274 Ähnlich stellt auch MARING heraus, dass Unternehmen „,nicht den Selbstzweckcharakter wie natürliche Personen [haben]. Man hat trotzdem Parallelen zum moralischen Handeln von Personen, aber eine Korporation ist keine Person im moralischen Sinne.“275 Dennoch können nach NEUHÄUSER Unternehmen als Verantwortungsträger aufgefasst werden, da diese mehr Handlungsmöglichkeiten und Ressourcen als Individuen alleine besäßen und auch als Einheiten wahrgenommen würden, welche gemeinsam Beschlüsse fassen, auf die sich auch die Stakeholder (z. B. gerade die Mitarbeiter) in ihrem Handeln ausrichteten ${ }^{276}$ : „Insofern wäre es vielleicht möglich, die Diskussion zum Begriff der Personalität über Bord zu werfen und stattdessen nur davon zu sprechen, dass Unternehmen Akteure sind, weil sie Intentionen haben.“277 NEUH'AuSER verschiebt damit evidenterweise die Begründung der Verantwortung von der metaphysischen Subjektbegründung hin zur faktischen gesellschaftlichen Wahrnehmung von Unternehmen als intentional handlungsfähigen Einheiten. Dieser Auffassung soll im Weiteren gefolgt werden. Jedoch ist noch zu klären, weshalb Unternehmen dann auch motiviert sein sollten, dem Anspruch, Verantwortung zu tragen, nachzukommen. In diesem Sinne stellt auch NEUHÄUSER in Bezug auf Unternehmen berechtigt fest: „Sie mögen dazu zwar grundsätzlich fähig sein, sind aber offensichtlich kaum motiviert, ethisch zu handeln. Woher aber soll diese

\footnotetext{
${ }^{273}$ Soares, C. (2003), S. 147. Auch STAHL betont in diesem Sinne: „Korporationen sind mehr als die Summe ihrer Mitglieder.“ (Stahl, B. C. (2000), S. 229).

${ }^{274}$ Neuhäuser, C. (2011a), S. 108. Vgl. auch Aras, G. / Williams, P. (2017), S. 16; De George, R. T. (1986), S. 61.

275 Maring, M. (2001b), S. 117.

${ }^{276}$ Vgl. Neuhäuser, C. (2011a), S. 90 und S. 164-165.

${ }^{277}$ Neuhäuser, C. (2011a), S. 103-104.
} 
Motivation praktisch kommen?“278 Dieser Frage wird sich die Arbeit im Folgenden widmen. Dabei wird, beruhend auf einer systemtheoretischen Konzeption des Unternehmens gezeigt, dass sehr wohl gute, für die Existenzsicherung des Unternehmens intrinsisch bedeutsame, Gründe existieren, Verantwortung im Sinne des „Rede- und Antwortstehens“ zu übernehmen, ohne wie bei SUCHANEK oder WIELAND auf traditionelle ökonomische Kategorien zurückzufallen. ${ }^{279}$ Des Weiteren wird allerdings auch nicht nur auf die reine Wirksamkeit von Moralappellen vertraut. Darüber hinaus kann ein solcher Ansatz aber auch als erkenntnisstiftend für die Implikationen im Rahmen der Digitalen Transformation erachtet werden, in welcher technische, computerisierte Entscheidungsunterstützungssysteme als cyber-physische Systeme zunehmende Bedeutung erfahren. ${ }^{280}$

\subsection{Unternehmen als sozio-technische Systeme - Forschungsgegenstand einer systemtheoretisch fundierten Unternehmensethik}

Wie die vorigen Ausführungen darlegten, scheint es für die wirtschaftsethische Forschung sinnvoll, sich intensiviert mit der Verantwortung von Unternehmen zu beschäftigen, wobei der Weg über eine metaphysische Subjektzuschreibung eine Reihe von (weitgehend ungeklärten) Problemen impliziert. Diese Fragen sollen hier nicht entschieden, sondern vielmehr zum Anlass genommen werden, die Möglichkeit einer subjektunabhängigen Unternehmensethik ${ }^{281} \mathrm{zu}$ untersuchen.

${ }^{278}$ Neuhäuser, C. (2011a), S. 181. Ähnlich stellen auch AßLÄNDER und BRINK fest, dass bisher ,eine offensichtliche Lücke [klaffe] zwischen der praktischen Frage, wie Unternehmen den unterschiedlichen Aspekten ihrer Verantwortung inhaltlich gerecht werden können, und der theoretischen Frage, wie sich eine Pflicht zur [.] Verantwortungsübernahme ethisch begründen lässt [...].“ (Aßländer, M. S. / Brink, A. (2008), S. 109, in der Quelle ebenfalls herv.).

${ }^{279}$ Da SUCHANEK auf den Begriff der Investition rekurriert, könnte man hier von einem strategischen Kalkül sprechen, während die Überlegungen WIELANDS zu einer Transaktionskostensenkung durch Moralimplementierung einen eher operativen Charakter aufweisen. Aus unternehmensethischer Perspektive fehlt damit jedoch eine genuin normative Reflexionsebene, welche allen strategischen Überlegungen wie operativen Implementierungsfragen vorausgeht. Vgl. hierzu detailliert die Ausführungen in Abschnitt 4.4.

${ }^{280}$ In diesem Sinne kann auch mit FETZER konstatiert werden: „Nicht selten interagieren aber in [Unternehmen] nicht nur Menschen mit Menschen, sondern auch Menschen mit Maschinen und sogar Maschinen mit Maschinen." (Fetzer, J. (2004), S. 68).

${ }^{281}$ Mit dieser Formulierung sei nochmals hervorgehoben, dass die weiteren Ausführungen nicht darauf abzielen, zu postulieren, dass Individuen wie auch Kollektive in keinem Falle 
Hieran anknüpfend soll im Weiteren Unternehmensethik im Sinne einer doppelten Subjektunabhängigkeit konzeptualisiert werden. Eine originäre Mesoethik muss dabei erstens in ihrem Betrachtungsgegenstand die Summe der Individuen übersteigen, d. h. es darf sich nicht um eine verdeckt-aggregierte Individualethik handeln, welche zweitens auch ohne metaphysischen Subjektstatus die Notwendigkeit von Ethik ohne Rückfall auf rein ökonomische Begründungsstrukturen oder reine Moralappelle darzulegen vermag. Dies gelingt mittels einer systemtheoretischen Konzeption, welche das Unternehmen als sozio-technisches System $^{282}$ rekonstruiert, wie die nachfolgenden Ausführungen zeigen. ${ }^{283}$

\subsubsection{Systemtheoretische Grundlagen}

Beschäftigt man sich näher mit dem Begriff der Systemtheorie, so zeigt sich recht schnell, dass bis heute nicht eine einzige Systemtheorie existiert. Vielmehr repräsentiert diese einen interdisziplinären Sammelbegriff, welcher als fruchtbares Analyseinstrument in diversen Disziplinen und Anwendungsebenen aus konzeptioneller Sicht Erkenntnisgewinne ermöglicht. ${ }^{284}$ So lassen sich folgerichtig je nach betrachteter wissenschaftlicher Disziplin auch verschiedene Systemdefinitionen auffinden, die jedoch inhaltlich im Detail stark differieren können. Allen Ansätzen ist jedoch der Grundgedanke gemein, einerseits holistisch sowohl die Eigenschaften des Ganzen, wie auch andererseits analytisch dessen Interaktionen zu betrachten ${ }^{285}$, wobei zu beachten ist, dass die Beziehungen zwischen den

mehr Subjekte im aufklärerischen Sinne sein könnten, vielmehr soll mit dem systemtheoretischen Ansatz ein Weg beschritten werden, welcher ohne inhaltlich-metaphysische Vorannahmen hinsichtlich eines solchen Subjektstatus die Notwendigkeit der Verantwortungsübernahme aus Sicht von Unternehmen intrinsisch begründet.

${ }^{282}$ So stellt in diesem Zusammenhang auch WoLF treffend fest: „Die mit der Systemtheorie arbeitenden Organisations-, Management- und Unternehmensführungswissenschaftler gehen davon aus, dass Unternehmen in hohem Maße durch die [..] allgemeinen Kernmerkmale von Systemen charakterisiert sind.“ (Wolf, J. (2013), S. 165).

283 Die Ausführungen der nachfolgenden Unterkapitel beruhen grundlegend auf Lingnau, V. / Beham, F. / Fuchs, F. (2022); Lingnau, V. / Beham, F. / Fuchs, F. (2020).

${ }^{284}$ Vgl. Sedlacek, K.-D. (2010), S. 11; Luhmann, N. (1994), S. 15; Müller-Merbach, H. (1992), S. 854. Dies gilt auch insbesondere für die sozialwissenschaftliche Systemforschung. Vgl. hierzu Scherm, E. / Pietsch, G. (2007), S. 103.

285 Vgl. Ropohl, G. (2016), S. 96. 
Elementen neue Eigenschaften der Gesamtheit hervorbringen, welche die Eigenschaften der konstituierenden Elemente übersteigt. ${ }^{286}$ Diese Erkenntnis findet sich bereits klassisch bei ARISTOTELES, welcher in diesem Sinne als Urvater systemtheoretischer Betrachtungen gelten kann. Auf ARISTOTELES geht daher auch der vielfach in der Literatur rezipierte Ausspruch zurück, dass das Ganze stets mehr ist als die Summe seiner Bestandteile. ${ }^{287}$ Dieser Zusammenhang lässt sich wiederum durch die Etymologie der deutschen Bezeichnung "System“ vergegenwärtigen, die auf dem griechischen $\sigma u ́ \sigma \tau \eta \mu \alpha$ (sýstema) beruht, welches „,das aus mehreren Teilen zusammengesetzte und geordnete Ganze“288 bezeichnet.

Systemisches Denken an sich stellt dabei keine genuin neuere Entwicklung dar und ist prinzipiell, wie aufgezeigt wurde, ein ideengeschichtliches Grundkonzept der großen philosophischen Systeme von ARISTOTELES, KANT oder HEGEL. ${ }^{289}$ So stellt auch der Begründer der allgemeinen Systemtheorie LUDWIG VON BERTALANFFY fest: „In a certain sense it can be said that the notion of system is as old as European philosophy. “290 Ebenso betont auch WOLF: „Die für sie charakteristische Sichtweise ist mindestens genauso alt wie die Philosophie des Abendlandes.“"291 Auch im Ingenieurwesen ist seit jeher systemisches Denken fundamental zum Entwurf von Maschinen gewesen, ${ }^{292}$ auch wenn diese Bezeichnung noch nicht immer geläufig gewesen sein mag. Der Anspruch fachübergreifende, d. h. Metaerkenntnisse, über Systeme per se zu entwickeln, stellt jedoch noch eine relativ junge Entwicklung dar, welche ihren Ausgangspunkt in den Arbeiten von LuDWIG VON BERTALANFFY findet. ${ }^{293}$ VON BERTALANFFY

${ }^{286} \mathrm{Zu}$ dem in diesem Zusammenhang relevanten Begriff der Emergenz vgl. auch die Ausführungen in Abschnitt 4.3.3.2.

${ }^{287}$ Vgl. Aristoteles (2009), 1041b. Wortwörtlich lautet die zitierte Stelle in deutscher Übersetzung allerdings: „Dasjenige, was so zusammengesetzt ist, daß das Ganze eines ist, nicht wie ein Haufen, sondern wie die Silbe, ist nicht nur seine Elemente." Für einen Überblick über weitere Aphorismen vgl. in diesem Kontext Müller-Merbach, H. (1992), S. 856. Vgl. auch Mikulecky, D. C. (2010), S. 83.

${ }^{288}$ Fuchs, H. (1973), S. 35, welcher auf Hoffmeister, J. (1955), S. 598 rekurriert. Vgl. auch die Ausführungen der Neuauflage in Kirchner, F. et al. (2013), S. 651-652. Vgl. ähnlich auch Schöndorf, H. (2010d), S. 486-487. Aus diesem Ansatz wird bereits der instrumentelle Organisationsbegriff deutlich. Vgl. hierzu auch Abschnitt 4.3.3.2.

${ }^{289}$ Vgl. zu einem historischen Überblick auch Ropohl, G. (2012), S. 21-29; Ropohl, G. (2009), S. 71-75. Vgl. auch Ziemann, A. (2009), S. 469 sowie die dort angegebene Literatur.

290 Bertalanffy, L. v. (1972a), S. 407.

${ }^{291}$ Wolf, J. (2013), S. 161. Vgl. auch Fuchs, H. (1972), S. 47.

292 Vgl. hierzu Bertalanffy, L. v. (2015), S. 3-4.

${ }^{293}$ Wesentliche Beiträge zum Aufbau der Systemtheorie im 20. Jahrhundert lieferten des Weiteren der Informationstheoretiker CLAUDE SHANNON und der Kybernetiker NORBERT 
erkannte, dass das Konzept des systemischen Denkens nicht nur in einzelnen Fachdisziplinen wie der Biologie ${ }^{294}$ erkenntnisförderlich ist, sondern fruchtbare Erkenntnisse hinsichtlich einer Vielzahl von Phänomenen aus diversen konzeptionellen Perspektiven zu liefern vermag, welches wiederum zur Forderung der Entwicklung einer „allgemeinen“, d. h. fachübergreifenden ${ }^{295}$, Systemtheorie als Metakonzept führte. ${ }^{296}$ In diesem Sinne formulierte VON BERTALANFFY pointiert: „Es gibt Modelle, Prinzipien und Gesetze, die für allgemeine Systeme oder Unterklassen von solchen gelten, unabhängig von der besonderen Art der Systeme, der Natur ihrer Komponenten und der Beziehungen oder Kräfte zwischen diesen. Wir fordern daher einen neuen Wissenschaftszweig, genannt Allgemeine Systemtheorie." ${ }^{297}$ Demnach ist es aus einer solchen verallgemeinernden Metaperspektive auch ,gleichgültig, welcher Art die sie zusammensetzenden Elemente und die zwischen diesen bestehenden Beziehungen oder ,Kräfte“ sind. “298 Relevant ist vielmehr, wie auch die weiteren Ausführungen darlegen, dass Systeme grundsätzlich aus einer Menge geordneter Elemente bestehen, zwischen denen stärkere Wechselbeziehungen bestehen als zu anderen Elementen, wodurch gegenüber

WIENER. Vgl. hierzu grundlegend Wiener, N. (2013); Shannon, C. E. (1948) sowie im Überblick Sedlacek, K.-D. (2010), S. 11-15; Wilms, F. E. P. (2001), S. 46-48.

${ }^{294}$ So z. B. zur Feststellung, dass Lebewesen (bei VON BERTALANFFY als „offene Systeme“ bezeichnet) durch ein Fließgleichgewicht gekennzeichnet seien. Vgl. hierzu Bertalanffy, L. v. (2015), S. 155-160; Bertalanffy, L. v. / Beier, W. / Laue, R. (1977), S. 1-7 und S. 28; Bertalanffy, L. v. (1975), S. 43-44; Bertalanffy, L. v. (1972c), S. 38; Bertalanffy, L. v. (1969), S. 73; Bertalanffy, L. v. (1950a), S. 155; Bertalanffy, L. v. (1950b), S. 23.

295 So stellt in diesem Zusammenhang auch RopoHL fest: „Die Allgemeine Systemtheorie [.] ist, wie ihr Name sagt, nicht auf eine bestimmte Disziplin begrenzt, sondern fachübergreifend.“ (Ropohl, G. (2009), S. 71). Ähnlich stellt auch FUCHS fest, ,daß unterschiedliche Phänomensysteme formal gesehen vielfach isomorphe oder homomorphe Strukturen aufweisen, für welche allgemeine homologe Systemgesetze gelten [.].“ (Fuchs, H. (1972), S. 47).

${ }^{296}$ In diesem Zusammenhang hebt VON BERTALANFFY hervor: „Thus, concepts such as wholeness and sum, mechanisation, centralisation, hierarchical order, stationary and steady states, equifinality, etc., are found in different fields of natural sciences, as well as in psychology and sociology." (Bertalanffy, L. v. (1950a), S. 164). Die Systemtheorie könnte damit nach VON BERTALANFFY die Basis für eine „Unity of Science“ (Bertalanffy, L. v. (2015), S. 48; Bertalanffy, L. v. (1950a), S. 164) liefern. Vgl. hierzu auch Wolf, J. (2013), S. 159.

${ }^{297}$ Bertalanffy, L. v. (1972b), S. 21.

${ }^{298}$ Bertalanffy, L. v. (1972c), S. 31. Vgl. auch Bertalanffy, L. v. (1950a), S. 139: „There are principles which apply to systems in general, whatever the nature of their component elements or the relations or ,forces" between them." Vgl. ähnlich auch Bertalanffy, L. v. (2015), S. 37; Bertalanffy, L. v. (1969), S. 69. 
der Umwelt eine Systemgrenze entsteht. ${ }^{299}$ Durch diese Verallgemeinerung des Systemgedankens wurde es nun möglich, eine Vielzahl von Systemen, sowohl natürlichen als auch künstlichen Ursprungs, zu beschreiben. Folglich wurde im Laufe der Zeit nicht nur die allgemeine Systemtheorie selbst kontinuierlich verbessert, sondern diese Weiterentwicklung förderte auch die allgemeine Verbreitung systemischen Denkens an sich und übte bedeutenden Einfluss auf zahlreiche Fachdisziplinen aus, nun ebenfalls verstärkt systemische Paradigmen in ihr Forschungsprogramm zu integrieren. So finden sich heute neben einigen Beiträgen der Biologie zur Auffassung von Leben als systemisch modellierbarem Phänomen auch umfangreiche systemtheoretische Beiträge in der Physik oder dem Ingenieurwesen, welches beispielsweise Systeme vielfach im Kontext der Signal- oder Regeltechnik aufgreift. ${ }^{300}$ Auch im sozialwissenschaftlichen Bereich hat der systemtheoretische Ansatz eine intensive, aber auch kontroverse Rezeption gefunden. So konzipierten z. B. die bekannten Soziologen PARETO $^{301}$, PARSONS ${ }^{302}$ sowie insbesondere auch LUHMANN ${ }^{303}$ - trotz überaus unterschiedlicher konzeptioneller Zugänge - ihre Gesellschaftstheorien auf einem systemtheoretischen Fundament. Aber auch in der Betriebswirtschaftslehre findet der Systemansatz seit einigen Jahrzehnten zunehmende Beachtung. Hier gilt HANS ULRICH als einer der zentralen Wegbereiter bzw. Begründer der betriebswirtschaftlichen Systemtheorie im deutschsprachigen Raum. ${ }^{304}$ Obwohl seine späteren Ausführungen sich auf den betrieblichen Kontext fokussieren, können seine grundlegenden Überlegungen auch auf Systeme im Allgemeinen übertragen werden. ULRICH bezeichnet dabei ein System als ,eine geordnete Gesamtheit von Elementen, zwischen denen irgendwelche Beziehungen bestehen oder hergestellt

${ }^{299}$ Vgl. Rüegg-Stürm, J. (2003), S. 17-18. In diesem Sinne definiert VON BERTALANFFY ein System ,als eine Anzahl von in Wechselwirkung stehenden Elementen [...].“ (Bertalanffy, L. v. (1972c), S. 32).

${ }^{300}$ Vgl. z. B. die Ausführungen in Beucher, O. (2019); Puente León, F. / Jäkel, H. (2019); Röbenack, K. (2017); Reinschke, K. (2014); Rennert, I. / Bundschuh, B. (2013); Deutscher, J. (2012); Frey, T. / Bossert, M. (2008).

${ }^{301}$ Vgl. Pareto, V. (1976). So sind z. B. bei PARETo die „Moleküle des sozialen Systems [.] die Individuen [...].“ (Pareto, V. (1976), S. 265).

302 Vgl. Parsons, T. (1971); Parsons, T. (1968).

303 Vgl. Luhmann, N. (2017); Luhmann, N. (2009a); Luhmann, N. (1994).

${ }^{304}$ Vgl. Bardmann, M. (2019), S. 446-447; Schwaninger, M. (2016), S. 351; Vollmer, T. (2014), S. 17-18; Lattwein, J. (2002), S. 12. Die von ihm begründete St. Galler Schule hat hierbei einige bekannte Autoren hervorgebracht, welche das systemtheoretische Paradigma weiterentwickelt haben, so z. B. MALIK (vgl. bspw. die Ausführungen in Malik, F. (2015); Malik, F. (2009); Malik, F. / Probst, G. (1981)). 
werden können." ${ }^{305}$ Konstitutiv für das Systemkonzept nach ULRICH sind somit die vier Merkmale: Element, Beziehung, Ganzheit und Ordnung (Struktur) ${ }^{306}$, welches die folgende Abbildung 4.4 im Überblick aufzeigt.

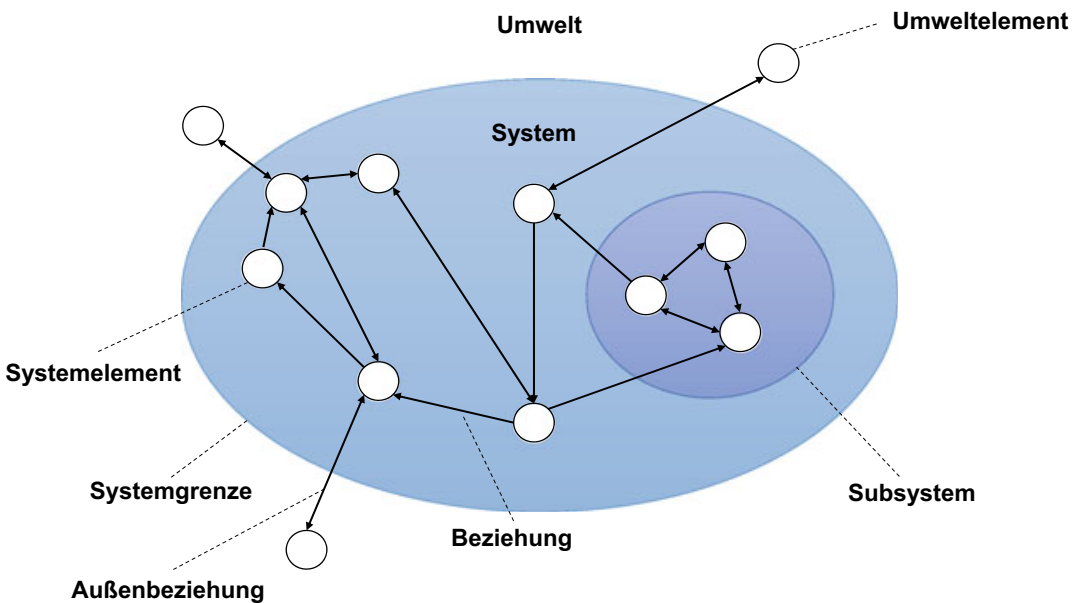

Abbildung 4.4 Konzeptioneller Überblick zum Systembegriff ${ }^{307}$

Unter einem Element wird dabei derjenige Bestandteil eines Systems verstanden, der nicht weiter zerlegt werden soll ${ }^{308}$ oder prinzipiell nicht weiter sinnvoll unterteilt werden kann. Demnach bildet das Element die kleinste zu betrachtende oder betrachtbare Einheit eines Systems. „Elemente sind somit die Komponenten eines Systems, also all das, was im wechselseitigen Zusammenwirken ein

305 Ulrich, H. (1970), S. 105, in der Quelle ebenfalls herv. Vgl. ähnlich auch Bertalanffy, L. v. (1972a), S. 417; Bertalanffy, L. v. (1972b) sowie die Ausführungen bei FUCHS, welcher konstatiert: „Trotz der Vielzahl der Systemdefinitionen lassen sich die für die Begriffsbildung wesentlichen Merkmale auf die Begriffe ,Elemente', ,Beziehungen“ und ,Eigenschaften“ zurückführen.“ (Fuchs, H. (1972), S. 49). Ebenso stellen BRUNNBERG und KIEHNE fest: „Wenn irgendwelche Beziehungen, Relationen oder Zusammenhänge zwischen bzw. unter den Elementen einer gegebenen Menge bestehen und zu erkennen sind, so sprechen wir von einem System, um nicht nur die Menge, sondern auch die Existenz dieser Beziehungen mit zum Ausdruck zu bringen.“ (Brunnberg, J. / Kiehne, R. (1972), S. 59).

306 Vgl. ähnlich auch Wolf, J. (2013), S. 159-161.

${ }^{307}$ In Anlehnung an Ulrich, H. (1984), S. 51 und S. 69.

308 Z. B. da die Struktur auf den unteren Stufen bereits bekannt oder für die Untersuchung unwesentlich ist. 
System konstituiert.“309 Beziehungen werden dagegen verstanden als ,,irgendwelche Verbindungen zwischen Elementen, welche das Verhalten der Elemente und das ganze System charakterisieren. Durch Beziehungsaufnahme oder Interaktion werden diese Beziehungen gewissermaßen aktiviert ${ }^{* 310}$, wodurch sich auch die Möglichkeit einer Gestaltung von Systemen ergibt. Unterhält das System direkte Beziehungen ${ }^{311}$ zum Umfeld, spricht man von einem offenen System. Gibt es diese Beziehungen nicht, spricht man dagegen von einem geschlossenen System. ${ }^{312}$ Während geschlossene Systeme auf der Grundprämisse basieren, dass lediglich eine intra-unit-Betrachtung vorliegt, bei der Veränderungen der Umwelt keinen direkten Einfluss auf systeminterne Spezifika haben können ${ }^{313}$, gehen offene Systeme von der gegensätzlichen Annahme aus, dass primär externe Umwelteinflüsse und -beziehungen (extra-unit-Betrachtung) als Erklärungsgrund für systeminterne Veränderungen anzusehen sind. Nach diesem Verständnis werden systemexterne Einflüsse als Input aufgenommen, gemäß gewisser Transformationsregeln verarbeitet und als Output wieder an die Umwelt abgegeben. Als Zwischenstufe zwischen offenen und geschlossenen Systemen findet sich in der Literatur das Konzept der autopoietischen bzw. selbstreferentiellen Systeme. Diese können, wie auch die nachfolgenden Ausführungen zeigen, sowohl als offene als

${ }^{309}$ Rüegg-Stürm, J. (2003), S. 18, in der Quelle ebenfalls herv.

${ }^{310}$ Ulrich, H. (1970), S. 109. Dabei können sich diese Beziehungen z. B. durch ,den Austausch von Energie, Materie und Information zwischen Elementen konkretisieren." (Fuchs, H. (1972), S. 49, in der Quelle ebenfalls herv.). Vgl. hierzu auch die Systematisierung der möglichen systemischen Beziehungen bei Fuchs, H. (1972), S. 49.

${ }^{311}$ Diese Beziehungen umfassen bei sozialen Entitäten den Austausch von Informationen oder (bei verknüpfter Information) Wissen, welches vielfach aufbereitet über die s. g. Sekundärwissensträger dem Management als Führungsunterstützung zur Verfügung gestellt wird. Vgl. hierzu auch Abschnitt 4.6.1.

${ }^{312}$ Vgl. Ulrich, P. / Fluri, E. (1995), S. 31. Was genau unter Geschlossenheit zu verstehen ist, differiert zwischen den Fachdisziplinen. Im physikalisch-technischen Sinne wird dabei zum Teil noch zwischen geschlossenen und abgeschlossenen Systemen unterschieden, wobei erstere keinen Materie-, aber Energieaustausch mit der Umwelt durchführen können, während letztere vollkommen von der Umwelt isoliert sind. Vgl. Baehr, H. D. / Kabelac, S. (2016), S. 14-15; Weidlich, W. (2016), S. 87; Weigand, B. / Köhler, J. / Wolfersdorf, J. v. (2016), S. 3-4; Dahm, K. / Visco, D. (2015), S. 13-14; Atkins, P. / Atkins, P. W. / de Paula, J. (2014), S. 64; Bertalanffy, L. v. / Beier, W. / Laue, R. (1977), S. 29-30; Fuchs, H. (1972), S. 52. In dieser Hinsicht ist im strengen Sinne wahrscheinlich nur das Universum ein abgeschlossenes System. Mit der aufgezeigten Definition orientiert sich die Arbeit an der Allgemeinen Systemtheorie, welche in der Regel einer Zweiteilung folgt.

${ }^{313}$ Vgl. Foerster, H. v. (1984b), S. 10-13. Vgl. hierzu auch die detaillierteren Überlegungen in Abschnitt 4.3.3. 
auch als geschlossene Systeme aufgefasst werden. ${ }^{314}$ Offen einerseits, da sie auf Austauschbeziehungen zur Umwelt angewiesen sind und auf diese auch adaptiv reagieren müssen. Geschlossen andererseits, da diese bei der Erhaltung des Systems auf sich selbst Bezug nehmen, ihre Struktur relativ autonom intern konstituieren und dabei eine eigene Identität entwickeln. Hieraus ,ergibt sich [.], daß selbstreferentielle Systeme auf dieser Ebene von ihrer Umwelt unabhängig und dementsprechend auch nicht für ihre innersystemischen Prozesse auf einen initi-

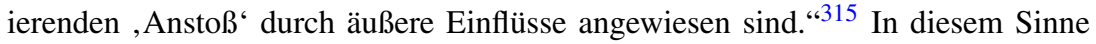
wird also ,[d]er Fokus der Betrachtung [..] auf die internen Differenzen gerichtet, die nun zum Referenzpunkt werden“316 (intra-unit-difference-Betrachtung). ${ }^{317}$

Systeme lassen sich grundsätzlich durch die Art sowie Intensität der Beziehungen zwischen den einzelnen Elementen konstituieren und als Ganzheiten betrachten. So liegt ein System vor, ,,wenn innerhalb dieser Gesamtheit ein größeres $\mathrm{Ma} ß$ von Interaktionen oder Beziehungen besteht als von der Gesamtheit nach außen. “318 Die von dieser Definition ausgeschlossenen Bestandteile bilden demnach die Umwelt des Systems, können jedoch potentiell wiederum Teile eines übergeordneten Supersystems repräsentieren. Konstitutiv für den Systembegriff ist in jedem Fall also die Vorstellung einer „Systemgrenze“, die eine Differenzierung von Innen (System) und Außen (Umwelt) ermöglicht. Insgesamt ergibt sich hieraus für das System und seine Umwelt eine absteigende hierarchische Gliederung in ein Supersystem, Systeme, Subsysteme und (als kleinste Einheiten) Systemelemente. Als Subsysteme werden dabei grundsätzlich Systeme niederer Ordnung bezeichnet, die als Systemelemente weiter aufgespalten werden können. Analog wird gegenläufig hierzu durch Beziehungen zwischen mehreren Systemen ein Supersystem gebildet.

314 Diese sind nach vON KROGH und Roos ,,simultaneously open and closed.“ (Krogh, G. v. / Roos, J. (1995), S. 33).

315 Liebig, O. (1997), S. 57.

${ }^{316}$ Liebig, O. (1997), S. 55.

${ }^{317}$ Vgl. Gralke, H.-J. / Scherm, E. (2012), S. 613-614; Ziemann, A. (2009), S. 475; Liebig, O. (1997), S. 52-57. Vgl. hinsichtlich einer Abgrenzung zwischen einer extra- und intra-unit-Betrachtung auch klassisch für die Soziologie Poggi, G. (1965), S. 285-293.

318 Ulrich, H. (1970), S. 108. Eine ähnliche Abgrenzung anhand der Beziehungen zwischen systeminternen und systemexternen Elementen schlägt auch WILLKE vor und bezeichnet Systeme als ,einen ganzheitlichen Zusammenhang von Teilen, deren Beziehung untereinander quantitativ intensiver und qualitativ produktiver sind als ihre Beziehung zu anderen Elementen. Diese Unterschiedlichkeit der Beziehung konstituiert eine Systemgrenze, die System und Umwelt des Systems trennt.“ (Willke, H. (2006), S. 251). 
Als letztes Merkmal ist schließlich die Ordnung (Struktur) eines Systems zu nennen, da die Ganzheit regelmäßig eine geordnete Struktur aufweist. ${ }^{319}$ So weisen die Bestandteile eines Systems ein Anordnungsmuster auf ${ }^{320}$, welches z. B. die räumliche oder hierarchische Anordnung der Elemente bzw. Subsysteme im System bestimmt oder auch die zeitliche Anordnung der Aktivitäten der einzelnen Systemelemente. ${ }^{321}$ Der Begriff der Ordnung wird in der Literatur wiederum recht intensiv diskutiert und mit unterschiedlichen Begriffskonnotationen versehen. Als klassischer Ansatz soll hierbei wiederum kurz auf die Überlegungen von ARISTOTELES zurückgegriffen werden, nach welchem sich eine Einheit bzw. ein System vom Chaos des reinen Gemenges dadurch unterscheidet, dass die Konfiguration der Beziehungen zwischen den Elementen nicht beliebig, sondern von Relevanz ist bzw. ,einen Unterschied macht ${ }^{\star 322}$. Als weitere Systematisierung können hierauf aufbauend mindestens zwei Stufen der Ordnung gefunden werden, welche wiederum mit den aristotelischen Begriffen der $\varphi v ́ \sigma ı \varsigma$ (phýsis) und $\tau \dot{x} \chi \nu \eta$ (téchne) $)^{323}$ in Verbindung gesetzt werden können. So kann in einem ersten Schritt auch eine natürlich-spontan entstandene Ordnung durch faktisch bestehende, dauerhafte Beziehungen zwischen Elementen (gerade im empirischen Kontext) als Ordnung gelten. Darüber hinaus ist es jedoch auch möglich, eine Ordnung der Elemente mittels Einsicht und Disposition, z. B. durch den Menschen, anhand bestimmter Kriterien (z. B. geometrische oder lexikografische Ordnung) herbeizuführen, welche ebenfalls durch die Bedeutsamkeit der Beziehungen zwischen den Elementen geprägt ist (z. B. im Rahmen einer bibliografischen Indizierung). Für alle Systemstrukturen gilt jedoch, dass diese einerseits von den Eigenschaften der Bestandteile (Elemente) sowie andererseits deren Beziehungen zueinander

${ }^{319}$ Vgl. Ulrich, H. (1970), S. 109. So stellt auch VON BERTALANFFY fest, dass es ,einsichtig [sei], daß der Begriff des Systems mit denen der Ordnung oder Organisation von Teilen zu höheren Einheiten nahe verwandt ist." (Bertalanffy, L. v. (1972b), S. 18).

${ }^{320}$ Dies entspricht dem Begriff der instrumentellen Organisation. Vgl. hierzu Abschnitt 4.3.3.2.

321 Vgl. Ulrich, H. (1970), S. 108.

322 Aristoteles (1989), 1024a 2-3.

${ }^{323}$ ARISTOTELES unterscheidet zwischen Natur ( $\varphi$ oı (phýsis)) und Kunstfertigkeit ( $\tau \dot{\chi} \chi \nu \eta$ (téchne)). Während erstere aus sich selbst heraus tätig ist, repräsentiert die Kunstfertigkeit ein Vermögen des Menschen, in die Welt erkennend und gestalterisch einzugreifen. Vgl. Aristoteles (2009), 1032a 7; Aristoteles (1989), 192b 8-193a 2. 
geprägt werden ${ }^{324}$ und sich im Zeitverlauf in gewissem Maße durchaus verändern können, solange die charakteristische Grundstruktur des Systems weiterhin bestehen bleibt. ${ }^{325}$ Unter der Grundstruktur eines Systems wird dabei allgemein die Menge notwendiger und charakteristischer Beziehungen zwischen den einzelnen Systemelementen verstanden, die das System als Ganzheit definieren. ${ }^{326}$ Dies bedeutet wiederum, dass eine Änderung der notwendigen Beziehungen (Grundstruktur) eines Systems notwendigerweise zu einem anderen System(typ) führt. ${ }^{327}$ Die aufgezeigten Überlegungen sind auch für die weiteren Ausführungen bedeutsam, da z. B. alle Unternehmen durch ihre spezifische Zwecksetzung der Wertschöpfung eine typische Grundstruktur aufweisen müssen, welche damit zeitlich invariant ist. Hierauf aufbauend können (und müssen zum Systemerhalt) außerhalb dieser grundlegenden Struktur auch organisationale Anpassungen an die Umwelt erfolgen (s. g. Aufbau- bzw. Adaptionsstruktur).

Nachdem nun die terminologischen Grundlagen des Systembegriffs dargelegt wurden, stellt sich die Frage nach einer Klassifikation möglicher Systemtypen. Eine solche, für die weiteren Ausführungen der Arbeit fruchtbare Taxonomie, wird im Nachfolgenden erörtert.

\subsubsection{Abgrenzung verschiedener Systemtypen}

Um die Vielfalt der möglichen Systeme handhabbarer zu machen, empfiehlt es sich, zuerst eine grundlegende Klassifikation der Systemtypen vorzunehmen. ${ }^{328}$

${ }^{324}$ In diesem Sinne konstatiert auch HASSENSTEIN: „Die Eigenschaften der Elemente und die Gesetze ihres Zusammenwirkens bedingen die Systemeigenschaften [...]." (Hassenstein, B. (1972), S. 33, in der Quelle ebenfalls herv.).

325 Vgl. hierzu auch Krogh, G. v. / Roos, J. (1995), S. 36; Maturana, H. R. (1985), S. 143-144. Es sei angemerkt, dass vON KROGH und RoOs sowie MATURANA, aber auch andere Autoren, anstelle der Bezeichnung als Grundstruktur häufig den Terminus der „Organisation“" gebrauchen, welcher auf den Organisationsbegriff im instrumentellen Sinne rekurriert, der wiederum die Organisiertheit einer Einheit bezeichnet. Vgl. hierzu auch detailliert die begriffliche Abgrenzung in Abschnitt 4.3.3.2.

${ }^{326}$ Vgl. Maturana, H. R. (1985), S. 139; Varela, F. J. (1984), S. 25; Maturana, H. R. / Varela, F. J. (1980), S. 77.

${ }^{327}$ So stellen auch VON KROGH und RoOs fest: „If the organization of a system as a whole changes the system in itself will change and form a new system with a new identity." (Krogh, G. v. / Roos, J. (1995), S. 36). Die Grundstruktur eines Systems kann damit als Basis dienen, im Detail möglicherweise verschiedene Systeme zu einer Systemklasse zusammenzufassen. 328 Dies entspricht, wie im Weiteren dargelegt wird, der dritten Welt von POPPER, also der Schaffung von wissenschaftlichen Aussagensystemen über die Systemtheorie. 
Zur Abgrenzung verschiedener Systemtypen hat die Literatur eine Fülle an differenzierten Ansätzen hervorgebracht. ${ }^{329}$ So resümiert auch SCHENK, dass zur Klassifikation von Systemen ,zahlreiche Systemeigenschaften als Unterscheidungskriterien zur Verfügung [stehen]. “330 Ebenso konstatiert auch FUCHS: „Der Begriff ,System ' wird in verschiedenen Wissensbereichen zur Beschreibung und Kennzeichnung unterschiedlicher Sachverhalte benutzt." 331 Betrachtet man die vielfältigen in der Literatur vorgeschlagenen Systematisierungsansätze, so wird deutlich, dass vielfach eine Unterscheidung zwischen künstlichen und natürlichen Systemen vorgenommen wird. ${ }^{332}$ Allerdings zeigt sich ebenso, dass eine solche Differenzierung meist nur eingeführt, allerdings selten konzeptionell fundiert begründet wird. So fehlt allgemein bei vielen Abgrenzungsversuchen bzw. Systematiken eine dezidierte konzeptionelle Grundlegung der genutzten Hierarchien. Aus diesem Grunde wird der wissenschaftstheoretisch fundierten Begründung einer solchen Klassifikation ein besonderes Augenmerk geschenkt.

Wissenschaftstheoretisch folgt diese Arbeit dabei, wie im zweiten Kapitel dargelegt, grundsätzlich einer realwissenschaftlichen Perspektive ${ }^{333}$, welche das im Weiteren konzeptionell mittels des Systemparadigmas konzipierte Unternehmen als zentralen Forschungsgegenstand der Unternehmensethik erachtet und dieses folglich als realweltliche Entität hinsichtlich seiner Wirkungen in der Realität untersucht. Diese Überlegungen können dabei prinzipiell mit der Klassifikation POPPERS in Verbindung gebracht werden, welcher zwischen drei Sub-Universen ${ }^{334}$ bzw. drei Welten unterscheidet. Im Rahmen dieser Überlegungen differenziert POPPER zwischen einer physikalischen Welt der materiellen Dinge und Prozesse, einer Welt des Bewusstseins und einer Welt objektivierter Gedankeninhalte. ${ }^{335}$ Hieran werden nun die weiteren Ausführungen anknüpfen,

${ }^{329}$ Vgl. für weitere Klassifikationsmöglichkeiten von Systemen Bardmann, M. (2019), S. 130; Ropohl, G. (2012), S. 91; Dangelmaier, W. (2003), S. 33-37; Schenk, H.-O. (1991), S. 85; Gal, T. / Gehring, H. (1981), S. 9.

330 Schenk, H.-O. (1991), S. 85.

${ }^{331}$ Fuchs, H. (1972), S. 48.

${ }^{332}$ Vgl. z. B. Schauer, R. (2019), S. 25; Peters, S. / Brühl, R. / Stelling, J. N. (2005), S. 17; Eberhardt, S. (1998), S. 24; Koller, R. (1998), S. 24; Löffelholz, J. (1980), S. 79; Oberkampf, V. (1976), S. 44; Baetge, J. (1974), S. 63; Wegner, G. (1969), S. 19. Vgl. auch Ropohl, G. (2012), S. 89-90, welcher jedoch postuliert, dass alle Systeme menschliche Konstruktionen darstellen.

${ }^{333}$ Vgl. hierzu Abschnitt 2.6 sowie grundlegend auch Lingnau, V. et al. (2018).

${ }^{334}$ Vgl. zu diesem Terminus Popper, K. R. (1980), S. 143.

335 Vgl. Popper, K. R. / Eccles, J. C. (1981), S. 38; Popper, K. R. (1980), S. 143-145; Popper, K. R. (1979), S. 74 und S. 106-107. Eine ähnliche Systematisierung wurde in einem 
wobei allerdings der erste Begriff im betriebswirtschaftlichen Kontext adaptiert und konkretisiert werden muss, da das ursprüngliche Modell aus dem natur- und nicht dem wirtschafts- bzw. sozialwissenschaftlichen Kontext stammt. So nehmen für die weiteren Ausführungen soziale, aber auch technische Systeme eine bedeutsame Rolle ein, welche aus realwissenschaftlicher Perspektive ${ }^{336}$ ebenfalls eine reale Existenz beanspruchen können, d. h., eben nicht nur individuelle Bewusstseinskonstruktionen darstellen. Analog zur Einordnung von Materie und Energie in die erste Welt der Klassifikation POPPERS erscheint es ersichtlich, dass neben einer materiellen Grundlage technischer, aber auch sozialer Systeme (Individuen als physische Einheiten), die Beziehungen - ähnlich zur Energie als Potential im physikalischen Kontext - in ihrer realweltlichen Wirkung einer Untersuchung zugänglich sind. Dies gilt dann wiederum auch für sozio-technische Systeme, d. h. Einheiten, welche diese beiden Systemtypen kombinieren. Diese sind folglich der realen Sphäre zuzuordnen, insofern die Phänomene dieser Systeme prinzipiell realwissenschaftlich analysierbar sind.

Akzeptiert man einmal einen solchen ontologischen Standpunkt, so ist dennoch klar, dass diese Systeme von Individuen nur gefiltert bzw. rekonstruiert wahrgenommen werden können. Im Gegensatz zu radikalen Konstruktivisten (wie MATURANA und VARELA ${ }^{337}$ ) sei jedoch in einem gemäßigten Sinne davon ausgegangen, dass eine vom individuellen Bewusstsein unabhängige Außenwelt existiert, deren Eigenschaften und Wirkungen ein Beobachter (zumindest in Teilen) begreifen kann. Dabei ist es fraglos plausibel, wie Konstruktivisten vielfach

erstmals 1919 erschienenen Zeitschriftenbeitrag von FREGE vorgenommen. Vgl. Frege, G. (1986), S. 40-44. Die grundlegende Idee einer Dreiteilung der Welt lässt sich dabei bis in die klassische Antike zurückverfolgen. Vgl. Czichos, H. (2019a), S. 64. Zur Interaktion der drei Welten vgl. Eccles, J. C. (1994), S. 3. Eine weitere Klassifikation differenziert zudem häufig zwischen materiellen und logischen bzw. geistigen (Begriffs-)Systemen. Vgl. z. B. Schauer, R. (2019), S. 25; Santozki, U. (2006), S. 367; Gal, T. / Gehring, H. (1981), S. 9; Brunnberg, J. / Kiehne, R. (1972), S. 60; Fuchs, H. (1972), S. 48; Kiehne, R. (1969), S. 1-2.

${ }^{336}$ Die Sozialwissenschaften werden häufig, trotz anderer existierender Strömungen innerhalb der Disziplinen, den Realwissenschaften zugeordnet. Vgl. z. B. Helfrich, H. (2016), S. 6; Kornmeier, M. (2007), S. 14-15; Raffée, H. (1995), S. 23; Busse von Colbe, W. / Laßmann, G. (1991), S. 2). Ontologisch zugrunde liegt dabei die (letztlich metaphysisch begründete) Annahme, dass eine Welt außerhalb des Beobachters existiert, welche von dessen Existenz unabhängig ist. Vgl. Esser, H. (1999), S. 53.

${ }^{337}$ Diese haben den Begriff „,radikal“ zwar in ihrem Schrifttum nicht selbst verwendet, werden inhaltlich in der Literatur jedoch vielfach diesem Paradigma zugerechnet. Vgl. Martinsen, R. (2014), S. 19. Vgl. auch Müller, K. H. (2015), S. 247; Töpfer, A. (2012), S. 123; Luhmann, N. (2009b), S. 31 und S. 54; Kolb, A. (2006), S. 274; Schmidt, S. J. (1991), S. 12. Vgl. in diesem Zusammenhang auch die Ausführungen bei Maturana, H. R. / Varela, F. J. (2012), S. 31. 
betonen - und dies würden viele Realisten auch kaum bestreiten, dass unser Geist als autopoietisches System ${ }^{338}$ stets und ausschließlich eine Rekonstruktion der realen Außenwelt zu bilden vermag, wodurch wir ersichtlicherweise keinen direkten Zugang zum „Ding an sich“339 besitzen, sondern nur rekonstruierte Abbilder $^{340}$ hiervon bewusst wahrnehmen und begreifen können. Dennoch sei hier davon ausgegangen, dass diese Außenwelt als unabhängig vom Beobachter existent angenommen werden kann. ${ }^{341}$ Diejenigen Individuen wiederum, welche mittels ihrer psychischen Systeme ${ }^{342}$ diese Rekonstruktion durchführen, können auch gemäß der Klassifikation POPPERS als eigener Systemtyp aufgefasst werden. ${ }^{343}$ Schließlich können drittens objektivierte Geistesinhalte als eigener Systemtypus unterschieden werden. Hierzu gehören beispielsweise sämtliche wissenschaftliche Systeme (so auch diese Ausführungen), welche durch ihr wissenschaftliches Generierungsprinzip ${ }^{344}$ gekennzeichnet sind und auch wiederum zur Gestaltung realweltlicher Systeme genutzt werden können. Wichtig ist hierbei, dass diese „Welten“ nicht isoliert voneinander bestehen, sondern sich gegenseitig beeinflussen können. ${ }^{345}$ Ein Beispiel im Kontext empirischer Aussagen ${ }^{346}$ möge dies verdeutlichen: So bilden bspw. Individuen mit ihrem psychischen System (Bewusstsein) Hypothesen über die Realität und testen ihre Hypothesen an der Erfahrung. Führt man dieses Prinzip nach wissenschaftlichen Standards durch ${ }^{347}$, erhält man ein Set an bestätigten (und ggf. falsifizierten) Hypothesen, welche zu

338 Siehe hierzu detailliert den nachfolgenden Abschnitt 4.3.3.

${ }^{339}$ Dieser Gedanke bzw. diese Formulierung geht bekanntermaßen auf KANT zurück. Vgl. Kant, I. (1968c), B 29-32.

${ }^{340}$ Diese sind in Anlehnung an KANT Rekonstruktionsleistungen des Verstandes.

${ }^{341}$ Vgl. hierzu auch Helfrich, H. (2016), S. 80. Vgl. in diesem Kontext auch die umfangreiche Diskussion verschiedener Positionen in Freede, J. (2008), S. 64-83.

342 Dies ist ein Terminus, welchen auch LUHMANN verwendet. Vgl. Luhmann, N. (1994), S. 16.

${ }^{343}$ Dies wird auch dadurch deutlich, dass z. B. das individuelle Bewusstsein selbst nicht gemessen werden kann, sondern nur in einem gewissen Zusammenhang stehende biochemische Prozesse.

${ }^{344} \mathrm{Zu}$ den Anforderungen wissenschaftlicher Aussagen vgl. Bardmann, M. (2019), S. 6465.

345 Vgl. Popper, K. R. / Eccles, J. C. (1981), S. 36-38; Popper, K. R. (1980), S. 150.

${ }^{346}$ Darüber hinaus existieren auch weitere Aussagearten, welche bspw. formaler Natur sein können. Solche z. B. in der Mathematik oder Logik auftretenden Aussagearten benötigen keine empirische Prüfung an der Realität, sondern vielmehr eine Konsistenz bzw. Widerspruchsfreiheit der Aussagen innerhalb eines gegebenen Axiomensystems. Vgl. hierzu nochmals die Ausführungen in Abschnitt 2.5.1.

${ }^{347}$ Vgl. hierzu grundlegend Kornmeier, M. (2007), S. 57-66. 
einem wissenschaftlichen Aussagensystem zusammengefasst die dritte Welt der objektivierten Gedankeninhalte ergeben. ${ }^{348}$ Diese Überlegungen fasst nochmals die nachfolgende Abbildung 4.5 zusammen.

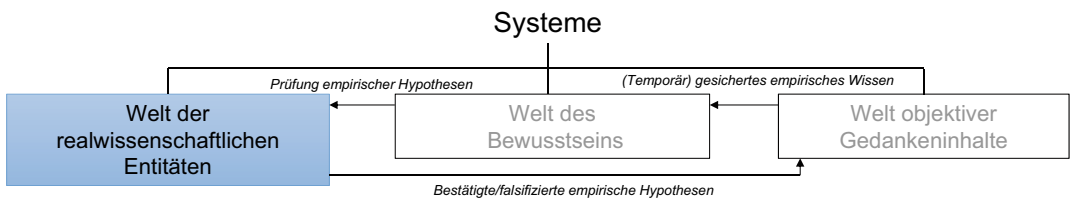

Abbildung 4.5 Grundlegende Systemklassifikation in Anlehnung an POPPERS Drei-Welten-Konzept

Wie die vorigen Ausführungen verdeutlichten, basieren die weiteren Überlegungen auf einem realwissenschaftlichen Fundament, so dass die im Weiteren zu untersuchenden sozialen und technischen Systeme der ersten Welt der realweltlichen Phänomene zuzuordnen sind. Zur weiteren Klassifikation seien nun in Anlehnung an ARISTOTELES ${ }^{349}$ die realweltlichen Entitäten in natürliche und künstliche Systeme differenziert. Während erstere spontan entstehen, sind künstliche Systeme von Individuen geschaffen und gestaltet. Unter natürlichen Systemen werden dabei in der Natur auftretende lebende (biotische), leblose (abiotische) Systeme sowie mögliche graduelle Mischformen zwischen lebenden und leblosen Systemen betrachtet. Künstliche Systeme werden dagegen von kunstfertigen Individuen $^{350}$ geschaffen und treten in dieser Form nicht in der freien Natur auf. Realexistierende Systeme sind dabei in der Regel in einem Kontinuum zwischen den beiden Extrempolen zu verorten. Sie weisen folglich teilweise spontan-emergente wie auch auf disponierende, gestalterisch tätige Lebewesen zurückzuführende Eigenschaften auf.

348 Vgl. hierzu auch Lingnau, V. et al. (2018), S. 4.

${ }^{349}$ Dieser unterscheidet, wie bereits aufgezeigt, zwischen Natur ( $\varphi$ oı (phýsis)) und Kunstfertigkeit ( $\tau \dot{\varepsilon} \chi \nu \eta$ (téchne)). Vgl. hierzu die Anmerkungen in Fn. 323.

${ }^{350}$ Dies dürften in der Regel Menschen sein. Inwiefern Kunstfertigkeit auch bei nichtmenschlichen Lebewesen existiert, sei im Rahmen dieser Arbeit ausgeklammert, aber nicht grundsätzlich verworfen (bspw. grundlegende Instrumentenbeherrschung bei bestimmten Spezies wie Schimpansen). 
Soziale Systeme können dabei im graduellen Sinne sowohl mehr oder minder emergent-natürlich entstanden, als auch bewusst und intentional geschaffen worden sein. Wie bereits ausgeführt wurde, weisen reale Systeme meist eine Mischung beider Eigenschaften auf, weshalb die graduelle Ausprägung in diesem Kontext besonders betont sei. So sind die meisten von Tieren gegründeten Staaten zwar eher den natürlichen Systemen zuzuordnen, weisen aber möglicherweise auch gewisse auf Dispositionen zurückzuführende Eigenschaften auf. Dagegen sind viele menschliche Sozialsysteme zwar aktiv vom Menschen gestaltet, die zugrunde liegende Sozialität ist aber auch der Natur des Menschen zugehörig. Aus diesem Grund können soziale Systeme sowohl Eigenschaften natürlicher als auch künstlicher Systeme aufweisen.

Technische Systeme sind dagegen stets durch die Kunstfertigkeit von Individuen (wie Menschen) mit Intention entstanden und damit den künstlichen Systemen zuzuordnen. $\mathrm{Zu}$ diesem gehören im Unternehmenskontext beispielsweise die Betriebsmittel wie Fertigungsanlagen oder auch betriebliche Informationsspeicher. Zur Gestaltung von technischen Systemen ist dabei vielfach Wissen zentral, wie dieses im Rahmen der wissenschaftlichen Forschung strukturiert generiert wird. Ein sozio-technisches System integriert schließlich sowohl Komponenten sozialer als auch technischer Systeme. Folgerichtig sind Systeme, in denen soziale Systeme unter Zuhilfenahme technischer Mittel tätig sind, als soziotechnische Systeme zu bezeichnen. Diese können, wie im Weiteren dargelegt wird, als theoretisches Fundament zur Beschreibung von Unternehmen als zentralem Forschungsgegenstand der Unternehmensethik herangezogen werden. ${ }^{351}$ Die nachfolgende Abbildung 4.6 fasst nochmals die vorigen klassifikatorischen Überlegungen in kompakter Form zusammen.

Dabei wird im Folgenden zuerst auf das soziale Teilsystem des Unternehmens eingegangen, welches sich, wie die weiteren Ausführungen zeigen werden, in der Regel als Organisation im institutionellen Sinne bzw. als hinreichend autopoietisches, d. h. selbsterhaltungsfähiges und zeitstabiles soziales System mit genuin eigenem Systemzweck und kollektivem Zielsystem manifestiert. Aufgrund der großen Bedeutung für das Unternehmen durch dessen dispositives, gestalterisches Potential sowie der inhaltlich kontrovers geführten Diskussion um dessen genaue Konzeption seien diese Systeme im Weiteren genauer untersucht.

\footnotetext{
${ }^{351}$ Die Ausführungen gelten dabei prinzipiell für alle Formen von Betrieben. Vgl. auch die grundlegenden Überlegungen in Lingnau, V. / Beham, F. / Fuchs, F. (2020).
} 


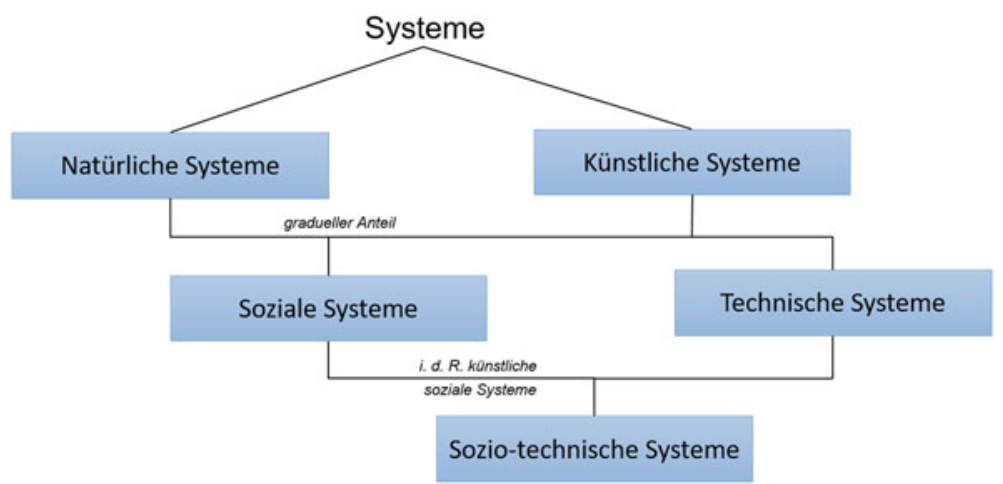

Abbildung 4.6 Einordnung der sozio-technischen Systeme zwischen natürlichen und künstlichen Systemen ${ }^{352}$

\subsubsection{Autopoiesis sozialer Systeme}

\subsubsection{Grundzüge der sozialwissenschaftlichen Autopoiesisdiskussion}

Beschäftigt man sich näher mit den Eigenschaften sozialer Systeme, so zeigt gerade die neuere sozialwissenschaftliche Forschung eine intensive Auseinandersetzung hinsichtlich der Frage auf, inwiefern diesen Systemen Eigenschaften der Lebendigkeit zugeschrieben werden können, ob dies für alle sozialen Systeme gelte und im Speziellen auch, wie diese systemische Eigenschaft konzeptionell zu begründen wäre. In diesem Zusammenhang erscheint folglich eine kurze Diskussion des Lebensbegriffs relevant, insbesondere, da dieser sowohl in der Philosophie ${ }^{353}$ als auch in der Biologie recht umfassend rezipiert und bis heute kontrovers diskutiert wird. So zeigt sich auch recht schnell, dass bis dato hinsichtlich des Begriffs des Lebens keine exakte, allgemeingültige Definition existiert. ${ }^{354}$

352 In Anlehnung an Lingnau, V. / Beham, F. / Fuchs, F. (2020), S. 20.

${ }^{353}$ Vgl. einführend Mahner, M. / Bunge, M. (2000), S. 135-136.

354 So konstatieren HACKER und KUMM: „Leben ist so vielfältig wie seine Definitionen.“ (Hacker, J. / Kumm, S. (2015), S. 19). Auch REECE ET AL. formulieren, dass ,sich das Phänomen, das wir Leben nennen, einer einfachen, in einem Satz formulierbaren Definition“ (Reece, J. B. et al. (2016), S. 2) entzieht. Vgl. hierzu auch Birch, C. (1999), S. 1. Ebenso prägnant resümiert auch PROSS: „Literally hundreds of definitions have been proposed over the years and there are few signs that the flow is abating [...]. To sum up [...] [the] , what is life“ riddle has remained unresolved." (Pross, A. (2016), S. 39 und S. 42). Analog resümiert 
Der Mangel einer vereinheitlichten grundlegenden Konzeption des Lebensbegriffs hat in der theoretischen Biologie zur Erarbeitung umfangreicher Merkmalslisten des Phänomens Leben geführt. Dabei wird z. B. neben einem Stoffwechsel, der Energieumwandlung und Evolution vielfach auch das Phänomen der Selbststeuerung bzw. Selbsterhaltung als charakteristisch für Lebewesen erachtet. ${ }^{355}$ Insbesondere letztere Begriffe konstituieren als Basiseigenschaft von Lebendigkeit einen fruchtbaren Ansatz für systemtheoretische Betrachtungen, welche über intuitive oder klassisch biochemisch geprägte Begriffsauffassungen von Leben hinausgehen. Als verallgemeinertes Abgrenzungsmerkmal zwischen lebenden und leblosen Systemen kann an dieser Stelle auf den Begriff der Autopoiesis zurückgegriffen werden. Dieser wurde ursprünglich im neurowissenschaftlichen Kontext von den Biologen MATURANA und VARELA im Rahmen der Untersuchung von Zellen geprägt, aber schon bald auf Lebewesen im Allgemeinen transferiert, wobei stets die Grundüberlegung darin bestand, das Kerncharakteristikum einer lebenden Einheit konzeptionell genauer herauszuarbeiten. Hierbei stellten die Autoren fest, dass das zentrale Merkmal eines lebenden Systems in der Fähigkeit besteht, sich aus sich selbst heraus zu erhalten und seine charakteristische Struktur bzw. Grundstruktur für eine gewisse Dauer zu stabilisieren, welches folglich auch die Reaktion bzw. Adaptivität an sich ändernde Umweltbedingungen inkludiert. ${ }^{356}$ Lebende Systeme besitzen folglich sowohl die Eigenschaft der

MAUSETH: „Despite the importance to biology of defining life, no satisfactory definition exists." (Mauseth, J. D. (2019), S. 16). In diesem Zusammenhang stellen wiederum BARON ET AL. fest, dass die heutige Forschung, ,den Versuchen ,Definitionen des Lebens ‘ zu finden, weitgehend entwachsen ist [...].“ (Baron, D. et al. (1996), S. 7). Ähnlich betonen auch RuIZMirazo, PERETO, und Moreno: „Definitions of life are highly controversial. And it is not just a question of lack of consensus among the different proposals formulated so far. Some authors are very skeptical about the actual possibility of grasping, in any scientifically relevant language" such a complex and multifarious phenomenon." (Ruiz-Mirazo, K. / Peretó, J. / Moreno, A. (2004), S. 323). Vgl. auch Bedau, M. A. (1992), S. 496. Dabei hebt jedoch LUISI hervor: „Die Frage nach der Definition des Lebens ist schwierig, es ist aber notwendig, sich damit zu befassen." (Luisi, P. L. (2002), S. 52).

355 Vgl. z. B. Campbell, N. A. et al. (2017), S. 51; Russell, P. J. / Hertz, P. E. / McMillan, B. (2017), S. 2; Fritsche, O. (2015), S. 3; Poeggel, G. (2013), S. 11; Schrauwers, A. / Poolman, B. (2013), S. 4-5; Sadava, D. E. et al. (2011), S. 3; Hickman, C. P. et al. (2008), S. 5-14; Wilson, D. L. (2006), S. 6; Koecke, H. U. / Emschermann, P. / Härle, E. (2000), S. 1; Davies, P. (1999), S. 33-36; Baron, D. et al. (1996), S. 8. Zu frühen Definitionen des Lebens, insbesondere in der klassischen Antike, vgl. grundlegend Kather, R. (2003); Lahav, N. (1999).

${ }^{356}$ Vgl. grundlegend Maturana, H. R. / Varela, F. J. (2012), S. 50-56; Maturana, H. R. / Varela, F. J. (1980), S. 78-84; Varela, F. J. / Maturana, H. R. / Uribe, R. (1974), S. 187189. Vgl. hierzu auch die Ausführungen bei Bedau, M. A. / Cleland, C. E. (2010a), S. 113; 
Selbstorganisationsfähigkeit als auch der Selbstreferenz ${ }^{357}$, auf welche sich die Prozesse der Selbsterhaltung beziehen. Die Bezeichnung als Autopoiesis fokussiert terminologisch genau auf diese Befähigung, wie bereits die Begriffsherkunft

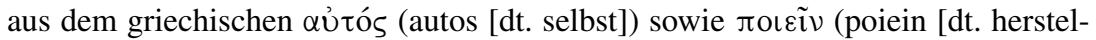
len, machen]) verdeutlicht. ${ }^{358}$ Unter Autopoiesis wird somit eine besondere Form der kontinuierlichen Selbsterzeugung und Selbsterhaltung verstanden, die als notwendige Eigenschaft lebender Systeme angesehen werden kann. ${ }^{359}$ In diesem Sinne stellt generalisiert und in Abgrenzung zu engeren biologischen Definitionen auch MALIK fest: „Unter ,Lebensfähigkeit“ ist vielmehr zu verstehen, dass Systeme, die die entsprechende Struktur aufweisen, sich an wandelnde Umstände in ihrer Umgebung anpassen, dass sie Erfahrungen aufnehmen und verwerten - also lernen, dass sie ihre Identität bewahren und sich entwickeln können."360 Der Terminus der Lebensfähigkeit weist dabei im Kern darauf hin, ,dass die spezifische Zustandskonfiguration, in welcher sich ein System faktisch befindet, auf unbestimmte Zeit aufrechterhalten werden kann. "361 Autopoietische Systeme lassen sich dabei den komplexen Systemen zuordnen ${ }^{362}$, welche wiederum nach SIMON „,irgendwo in der Grauzone zwischen Leben und Nichtleben“363 zu verorten sind.

Kneer, G. (1996), S. 312-313. In diesem Sinne konstatiert auch COLEMAN: „Autopoietic theory refers to systems that maintain their defining organization throughout a history of environmental perturbation and structural change [...].“ (Coleman, H. J. (1999), S. 36).

${ }^{357}$ Dabei stellt OESER fest, dass das Kernprinzip der Selbstreferentialität allerdings nicht notwendig auf biochemische Entitäten beschränkt ist: „Mit ,Selbstreferentialität“ ist jedoch ein universales Ordnungsprinzip gemeint, das nicht auf biologische Systeme oder überhaupt auf materielle Systeme beschränkt ist [...].“ (Oeser, E. (1988), S. 48).

358 Vgl. Staubmann, H. (2015), S. 276; Maturana, H. R. / Varela, F. J. (2012), S. 51; May, M. (2010), S. 110; Sedlacek, K.-D. (2010), S. 59; Kneer, G. (1996), S. 312; Kirsch, W. / Knyphausen, D. z. (1991), S. 78; Maturana, H. R. (1985), S. 141.

${ }^{359}$ Nach dieser Auffassung kann ein Lebewesen stets als autopoietisches System aufgefasst werden, wobei nach MATURANA und VARELA auch der Umkehrschluss zutrifft: ,,[A] physical system if autopoietic, is living. In other words, [...] autopoiesis is necessary and sufficient to characterize the organization of living systems." (Maturana, H. R. / Varela, F. J. (1980), S. 82, in der Quelle ebenfalls herv.). Vgl. ähnlich auch Varela, F. J. (1979), S. 17. Die Begründer der Autopoiesis setzen folglich die besondere Fähigkeit zur Selbstorganisation mit der Eigenschaft zu Leben gleich. Ähnlich stellen auch KIRSCH und ZU KNYPHAUSEN fest: „Damit ist aber ein autopoietisches System von vornherein auch schon ein System mit einer internen Dynamik, oder eben: ein lebendes System." (Kirsch, W. / Knyphausen, D. z. (1991), S. 79, in der Quelle ebenfalls herv.). Vgl. auch Fleischaker, G. R. (1990), S. 131.

${ }^{360}$ Malik, F. (2015), S. 73.

${ }^{361}$ Malik, F. (2015), S. 102.

362 Vgl. Gronau, N. (2006), S. 211; Fried, A. (2005), S. 49.

363 Simon, F. B. (2017), S. 19. 
Grundsätzlich gilt dabei, dass Komplexität ein essentielles, notwendiges Kennzeichen des Lebens repräsentiert, denn ,lebende Systeme sind extrem komplex und sie folgen eigenen Regeln, die manchmal den Naturgesetzen zu widersprechen scheinen.“364 Auch HEJL stellt in diesem Zusammenhang fest: „Lebende Systeme sind $[\ldots .$.$] nicht-triviale Systeme im Sinne Heinz von Foersters. “365$

Das Phänomen der Komplexität kann nun wiederum sowohl in Bezug auf die Umwelt als auch hinsichtlich des Systems selbst untersucht werden. Generell gilt dabei, dass Komplexität allgemein zu einer Unfähigkeit eines Systems führt, in einer gegebenen Entscheidungszeit die analysierbaren System- und Umweltzustände vollständig zu erfassen und zu verarbeiten, wobei wiederum zwischen der Eigenkomplexität des Systems sowie der Komplexität der Umwelt differenziert werden kann. Hinsichtlich des Begriffs der Komplexität wird im Weiteren auf die Überlegungen von GeRLING ${ }^{366}$ sowie LINGNAU und BRENNING ${ }^{367}$ zurückgegriffen, wonach zwischen systemunabhängigen- und systemspezifischen Komplexitätsmerkmalen unterschieden wird. Dabei können Systeme erstens durch einen steten Wechsel der Systemzustände (Dynamik) sowie eine starke Vernetztheit der Elemente gekennzeichnet sein, so dass diese als systemübergreifende Komplexitätsmerkmale aufgefasst werden können. Darüber hinaus treten zweitens jedoch auch im Rahmen autopoietischer Systeme durch die Fähigkeit zur Selbstorganisation und -beobachtung weitere Komplexitätsmerkmale hinzu. So sind diese Systeme häufig mit einer Vielzahl von potentiell konfligierenden

364 Schrauwers, A. / Poolman, B. (2013), S. 7. Vgl. ähnlich auch Pross, A. (2016), S. 4: „Living things are highly complex.“ Ebenso stellen auch BEDAU und CLELAND fest, dass ,life as we know it on Earth today is an extremely complex phenomenon [...].“ (Bedau, M. A. / Cleland, C. E. (2010b), S. 217).

365 Hejl, P. M. (1991), S. 309. VON FOERSTER unterscheidet dabei zwischen einer trivialen, deterministischen Maschine und einer nicht-trivialen Maschine, dessen Output nicht nur vom Input, sondern auch vom gegenwärtigen Eigenzustand des Systems abhängt. Vgl. hierzu Foerster, H. v. (2003), S. 309-313; Foerster, H. v. (1997), S. 37-41; Foerster, H. v. (1984a), S. 201-202; Foerster, H. v. (1984b), S. 8-13. Beispiele für solche Systeme sind neben lebendigen Systemen auch abiotische, hochkomplexe Systeme wie das Wetter, z. B. im Kontext des s. g. Schmetterlingseffekts oder auch das internationale Finanzsystem. Vgl. grundlegend auch Lingnau, V. / Beham, F. / Fuchs, F. (2020), S. 45.

366 Vgl. Gerling, P. G. (2007), S. 25.

${ }^{367}$ Vgl. Lingnau, V. / Brenning, M. (2018), S. 146-147; Lingnau, V. / Brenning, M. (2015), S. 457. 
Ansprüchen konfrontiert, die im Zielsystem zu berücksichtigen sind (Polytelie), welches wiederum eine funktionale Integrationsleistung ${ }^{368}$ erfordert. ${ }^{369} \mathrm{Da}$ autopoietische Systeme ihre Umwelt stets interpretierend und damit restringiert wahrnehmen, tritt zudem regelmäßig das Phänomen der Intransparenz auf, welches dazu führt, dass das System ,die Zustände einiger Systemvariablen nicht direkt feststellen kann.“370 Autopoietische Systeme müssen sich damit allgemein - erst Recht im Rahmen komplexer Umwelten - ,nicht nur an ihre Umwelt, sie müssen sich auch an ihre eigene Komplexität anpassen. Sie müssen mit internen Unwahrscheinlichkeiten und Unzulänglichkeiten zurechtkommen. Sie müssen Einrichtungen ${ }^{371}$ entwickeln [...], die abweichendes Verhalten reduzieren, das erst dadurch möglich wird, daß es dominierende Grundstrukturen gibt. “" ${ }^{372}$ In Bezug auf soziale Systeme kann dabei festgestellt werden, dass diese nicht nur als Einheit selbst komplex sind, sondern vielmehr auch komplexe Systemelemente enthalten. Inwiefern das Konzept der Autopoiesis jedoch auch auf soziale Systeme als Einheit selbst Anwendung finden kann, wird in der Literatur seit Längerem überaus kontrovers diskutiert, wobei sich auch in diesem Zusammenhang verschiedene Auffassungen entwickelt haben, die im Folgenden kurz skizziert seien. ${ }^{373}$

Bereits bei den Begründern des Konzepts, MATURANA und VARELA, zeigen sich dabei erste Überlegungen, welche einen potentiellen Transfer des originär biochemischen Autopoiesisbegriffs auf die soziale Ebene konzeptionell unterfüttern könnten. So differenzieren MATURANA und VARELA mehrere Ebenen der Autopoiesis. In einem ersten Schritt wird hierbei die Zelle als die grundlegendste autopoietische Einheit aufgefasst, welcher eine Autopoiesis erster Ordnung zugeschrieben wird. Hierauf aufbauend kann nun durch strukturelle Kopplung, d. h. einem reziproken Zusammenschluss bzw. einer dauerhaften Interaktion dieser grundlegenden Einheiten, bei gleichzeitiger Erhaltung der inneren Autonomie

\footnotetext{
${ }^{368}$ Diese findet im unternehmerischen Kontext durch das Management im funktionalen wie auch institutionellen Sinne statt. Vgl. hierzu auch die Ausführungen in Abschnitt 4.4.1.

${ }^{369}$ Hierbei ergibt sich hinsichtlich der Frage, welche Ansprüche in welchem Ausmaß zu berücksichtigen sind, ein zentraler Anknüpfungspunkt für ethische Überlegungen, wie auch die weiteren Ausführungen zeigen.

${ }^{370}$ Lingnau, V. / Brenning, M. (2015), S. 457.

${ }^{371}$ So z. B. auch das Management im institutionellen Sinne. Vgl. hierzu die Ausführungen in Abschnitt 4.4.1.

372 Luhmann, N. (1994), S. 56.

${ }^{373}$ Die folgende Klassifikation beruht maßgeblich auf den Überlegungen von Kirsch, W. (1997), S. 328-349. Vgl. auch den Überblick bei Holten, R. (1999), S. 152-165 und insbesondere Oeser, E. (1988), S. 43-49.
} 
bzw. Funktionslogik der Elemente, auf höherer Ebene ein Metazeller bzw. Organismus als autopoietische Entität zweiter Ordnung gebildet werden. Schließen sich diese Organismen (bzw. insbesondere menschliche Individuen) wiederum dauerhaft zusammen, so entsteht ein autopoietisches System dritter Ordnung. ${ }^{374}$ In diesem Zusammenhang hebt auch MATURANA hervor, dass (menschliche) Individuen das Kernelement eines sozialen Systems darstellen, denn es sei „konstitutiv für ein soziales System, daß seine Komponenten Lebewesen sind.“375 Die bisherigen Ausführungen legen dabei nahe, dass das Grundkonzept der Autopoiesis auch auf soziale Einheiten prinzipiell übertragbar sein könnte, welches in diesem Sinne auch von einigen Autoren interpretiert wurde. So betont z. B. klassisch BEER: „The outcome, to which I was admittedly predisposed because of my own work, says that any cohesive social institution is an autopoietic system because it survives, because its method of survival answers the autopoietic criteria [...]. “376 Auch GEYER und ZOUwEN stellen fest: ,[A]utopoiesis stresses the selforganizing, self-reproducing, and self-steering qualities of individuals and groups [...]. “377 Ebenso heben schließlich auch ZELENY und HUFFORD hervor, dass es ihre Intention sei, ,to propose, demonstrate, and argue that Varela et al.'s criteria as they are applied to a biological (living) system can be applied to other systems that we currently do not consider, living ${ }^{6}[\ldots]^{\text {‘378, }}$, womit die Autoren auch Sozialsystemen, wie z. B. Familien, einen autopoietischen Charakter zuschreiben. ${ }^{379}$

Zum Übertrag der Autopoiesis auf eine höhere Ebene muss dabei jedoch noch nach MATURANA und VARELA gelten, dass die Autopoiesis einer höheren Ordnung die Existenz der untergeordneten Ebenen bedingt. ${ }^{380}$ Dies hat allerdings zu kontroversen Diskussionen geführt, insbesondere hinsichtlich der Frage, inwiefern ein soziales System als die Autopoiesis von Individuen beeinflussend gedacht werden kann. Bei MATURANA findet sich in diesem Zusammenhang nun die Feststellung, dass ein soziales System stets als Ziel auch die Sicherung der individuellen Existenz seiner Mitglieder beinhalten müsse. So sei „eine Menge

\footnotetext{
${ }^{374}$ Vgl. Maturana, H. R. / Varela, F. J. (2012), S. 98 und S. 197; Maturana, H. R. / Varela, F. J. (1980), S. 107-111.

375 Maturana, H. R. (1991), S. 292-293.

376 Beer, S. (1980), S. 70.

${ }^{377}$ Geyer, R. F. / Zouwen, J. v. d. (2001), S. 6.

378 Zelený, M. / Hufford, K. D. (1992), S. 147, in der Quelle ebenfalls herv.

${ }^{379}$ Vgl. Zelený, M. / Hufford, K. D. (1992), S. 154-156.

380 So stellen MATURANA und VARELA fest: „An autopoietic system whose autopoiesis entails the autopoiesis of the coupled autopoietic unities which realize it, is an autopoietic system of higher order.“ (Maturana, H. R. / Varela, F. J. (1980), S. 109).
} 
von Menschen, die die Erhaltung des Lebens ihrer Mitglieder nicht als Teil der Definition ihres Funktionierens als System einschließt, kein soziales System.“381 Diese Position scheint, wie auch z. B. von KIRSCH interpretiert, eine Übertragungsfähigkeit des Autopoiesisgedankens auf die soziale Ebene als strukturelle Kopplung dritter Ordnung nahezulegen ${ }^{382}$ und dient KIRSCH selbst wiederum als Grundlage seiner eigenen gradualistischen Überlegungen, welche Autopoiesis als empirisch denkbaren, aber eher unwahrscheinlichen Grenzfall für soziale Systeme auffasst. $^{383}$

Eine klare Position gegen eine Übertragbarkeit der Autopoiesis auf soziale Entitäten hat dagegen VARELA eingenommen ${ }^{384}$, welcher die Auffassung eines Transfers der Autopoiesiskonzeption auf andere als biochemische Systeme deutlich kritisiert. Kernargument seiner Kritik ist dabei die Auffassung, dass Autopoiesis sich stets auf materielle Selbstproduktion der Elemente beziehen müsse, welche nur im biochemischen Sinne denkbar sei ${ }^{385}$, weshalb er für andere Kontexte die Einführung des Begriffs des „autonomen Systems“ vorschlägt, welches durch eine organisationale Geschlossenheit ${ }^{386}$ gekennzeichnet sei. ${ }^{387}$ Solche Systeme sind dabei nach VARELA ,a network of interactions of components that (i) through their interactions recursively regenerate the network of interactions that produced them, and (ii) realize the network as a unity in the space in which the components exist by constituting and specifying the unity's boundaries as a cleavage from the background [...].“388 Als wesentliches Differenzierungsmerkmal zur Konzeption autopoietischer Systeme müssen folglich bei autonomen Systemen die konstituierenden Elemente nicht selbst erzeugt werden, sondern

\footnotetext{
381 Maturana, H. R. (1991), S. 293.

382 Wobei sich MATURANA an anderer Stelle wiederum skeptisch hinsichtlich eines Gebrauchs des Autopoiesisbegriffs im Kontext sozialer Systeme zeigt, da diese schließlich „,nicht als ein Netzwerk der Produktion von Menschen konstituiert“ (Krüll, M. / Luhmann, N. / Maturana, H. R. (1987), S. 11) seien.

${ }^{383}$ Vgl. Kirsch, W. (1997), S. 328-329; Kirsch, W. / Knyphausen, D. z. (1991), S. 96.

${ }^{384}$ Auf diesen Sachverhalt mag auch die Formulierung bei BEER hinweisen, welcher feststellt, dass hinsichtlich seiner Interpretation des Autopoiesisbegriffs gelte, dass ,presumably at least one of the originators of autopoietic theory disagrees, or is less than sure ..." (Beer, S. (1980), S. 70).

${ }^{385}$ Vgl. Varela, F. J. (1981), S. 15. Vgl. auch Varela, F. J. (1979), S. 17, S. 41-42 und S. 51. 386 Vgl. hierzu detailliert Varela, F. J. (1984), S. 25-30.

${ }^{387}$ Vgl. Bourgine, P. / Varela, F. J. (1992), S. XI-XIII; Varela, F. J. (1981), S. 14-15; Varela, F. J. (1979), S. 53-57; Varela, F. J. (1978), S. 77-79.

388 Varela, F. J. (1981), S. 15.
} 
können auch von der Systemumwelt aufgenommen und dann spezifisch organisiert werden. In diesem Sinne könnte der Begriff des autonomen Systems folglich im graduellen Sinne zwischen den Extrempolen eines allopoietischen ${ }^{389}$ und autopoietischen Systems verortet werden, so dass Autopoiesis als (Extrem-) Ausprägung von autonomen Systemen aufgefasst werden könnte. ${ }^{390}$

Eine weitere bekannte Position in der sozialen Autopoiesisdiskussion stammt darüber hinaus von Peter HeJl. Dieser stimmt mit MATURANA und VAREla in einem ersten Schritt überein, Individuen als Elemente sozialer Systeme aufzufas$\operatorname{sen}^{391}$, folgt aber andererseits wiederum VARELA darin, dass das Autopoiesiskonzept nicht auf soziale Systeme übertragbar sei, welches er nach einer umfassenden Diskussion benachbarter Begrifflichkeiten ex definitione ausschließt. HEJL erarbeitet dabei im Rahmen konstruktivistischer Theoriebildung einen alternativen Ansatz. So postuliert er, dass soziale Systeme evolutionär durch die Notwendigkeit einer Koordination zwischen Lebewesen entstanden seien, welches eine Parallelisierung von kognitiven Zuständen voraussetze. Gerade beim Menschen, welcher sich auf Anpassungsfähigkeit spezialisiert habe, sei diese durch die Möglichkeit einer kollektiven Gefahrenabwehr, welche „mögliche Gefahren in

389 Allopoiesis bezeichnet dabei das Gegenteil von Autopoiesis, also eine Fremdherstellung und -organisation bzw. durch exogene Faktoren herbeigeführte Systemerhaltung (vgl. hierzu Varela, F. J. / Maturana, H. R. / Uribe, R. (1974), S. 188-189), wie sie sich insbesondere regelmäßig bei klassischen, technischen Systemen zeigt. In diesem Zusammenhang stellt auch ZIEMANN fest: „Deshalb sind autopoietische Systeme zwar geschlossen und autonom, aber keineswegs autark; ihre (Re-)Produktion kann nicht unabhängig von bestimmten materiellen und energetischen Umweltbedingungen vollzogen werden. Formelhaft gesagt: Autopoietische Systeme sind selbsterzeugend, selbsterhaltend und selbstorganisierend und deshalb operational, organisational und informationell geschlossen [...]. Dem gegenüber steht die Funktionsweise von, allopoietischen' Systemtypen, also von fremderzeugten und sich nicht selbst erhaltenden Systemen. Diese können ihre Elemente weder selbst erzeugen noch selbst organisieren, sondern bekommen diese aus der Umwelt zugeführt, um dann aus ihnen und durch sie einen spezifischen System-Output zu leisten." (Ziemann, A. (2009), S. 477). Vgl. hierzu auch Abschnitt 4.3.4.

390 Ähnlich auch VARELA: „Autopoiesis is a case of, and not synonymous with, organizational closure, and the autonomy of living systems is a case of, and not synonymous with, autonomy in general.“ (Varela, F. J. (1979), S. 57).

391 Vgl. Hejl, P. M. (1992a), S. 191; Hejl, P. M. (1992b), S. 270; Hejl, P. M. (1991), S. 319; Hejl, P. M. (1984), S. 61-62. 
Elemente des Zusammenhalts“392 transferierte, hochentwickelt. Die Parallelisierung der individuellen kognitiven Zustände zeige sich dabei ,,als physiologische Basis sozial erzeugter gemeinsamer Realitäten, von Sinn und Bedeutung [...].“393 Für soziale Systeme lehnt HEJL vor diesem konzeptionellen Hintergrund folglich die Bezeichnung als „autopoietisch“ explizit ab und schlägt vor, die aufgezeigte Parallelisierung mentaler Zustände als Synreferentialität sozialer Systeme zu bezeichnen. ${ }^{394}$

Während die bisherigen Ansätze noch immer das menschliche Individuum als Kernelement sozialer Systeme auffassen, verfolgt die durch NiKLAS LuHMANN begründete soziologische Theorietradition einen anderen konzeptionellen Zugang. So erachtet LUHMANN gerade nicht menschliche Individuen als Elemente sozialer Systeme, sondern vielmehr Kommunikationen, wobei LUHMANN einen spezifischen Kommunikationsbegriff zugrunde legt. ${ }^{395}$ Der Mensch ist damit folglich nicht mehr Teil des sozialen Systems, sondern dessen Umwelt zuzurechnen ${ }^{396}$, welche das soziale System lediglich anregen, aber nicht direkt beeinflussen kann (s. g. Perturbationen ${ }^{397}$ ). Darüber hinaus abstrahiert LuHMANN im Kontext der Autopoiesis vom physisch geprägten Begriff des Systemerhalts bzw. der materiellen Reproduktion und rückt das Konzept der „operationalen Geschlossenheit“ in den Fokus, die sich wiederum darin manifestiert, dass neue Operationen notwendig auf alten Operationen aufbauen müssten. Dies wird dann durch LUHMANN für soziale Systeme dahingehend interpretiert, dass diese grundsätzlich autopoietisch

${ }^{392}$ Hejl, P. M. (1991), S. 315. In diesem Zusammenhang stellt HEJL ebenfalls, wiederum recht nahe zu den Überlegungen MATURANAS, fest: ,Menschen leben sozial aus biologischen Gründen und können biologisch sein, wie sie sind, weil sie sozial leben. Gesellschaft als solche ist somit biologisch notwendig.“ (Hejl, P. M. (1991), S. 315, in der Quelle ebenfalls herv.). ${ }^{393}$ Hejl, P. M. (1991), S. 327.

394 Vgl. Hejl, P. M. (1992a), S. 195-196; Hejl, P. M. (1992b), S. 280; Hejl, P. M. (1991), S. 313-327; Hejl, P. M. (1984), S. 69-76.

395 Vgl. Luhmann, N. (2017), S. 44 und S. 246; Luhmann, N. (2008), S. 30-37; Luhmann, N. (1994), S. 240-241. LUHMANN kritisiert in diesem Zusammenhang die verbreitete Vorstellung, Kommunikation stelle eine reine Übertragung von Informationen dar, ,,weil sie zu viel Ontologie impliziert. Sie suggeriert, daß der Absender etwas übergibt, was der Empfänger erhält.“(Luhmann, N. (1994), S. 193). Nach LUHMANN besteht Kommunikation dagegen aus der Synthese von Information, Mitteilung und Verstehen. Vgl. Luhmann, N. (2017), S. 250; Luhmann, N. (2009a), S. 361; Luhmann, N. (2008), S. 111; Luhmann, N. (1997), S. 21; Luhmann, N. (1994), S. 193-201.

396 So sei es nach LUHMANN auch ,,völlig klar, daß Menschen nicht Teile oder Elemente sozialer Systeme sein können. [...] Es kann sich nur um Kommunikation handeln." (Luhmann, N. (2008), S. 30).

${ }^{397}$ Vgl. hierzu Maturana, H. R. / Varela, F. J. (1980), S. 81. 
seien, da Kommunikationen stets und ausschließlich durch Kommunikationen entstünden, wodurch ein hohes Maß an Abstraktheit, aber auch begrifflicher Schärfe innerhalb der Konzeption gewonnen wird. Durch zahlreiche Neufassungen von Begriffen kommt LUHMANN schließlich zur provokanten These, dass nicht der Mensch selbst kommuniziere, sondern lediglich die Kommunikationen zwischen den Menschen: ,[.] Menschen können nicht kommunizieren, nicht einmal ihre Gehirne können kommunizieren, nicht einmal das Bewußtsein kann kommunizieren. Nur die Kommunikation kann kommunizieren. “398 In LUHMANNS Werk ist folglich die Autopoiesis sozialer Systeme ex definitione gegeben.

An LUHMANNS Werk schließt sich wiederum aus rechtswissenschaftlicher Sicht GunTHER TeUbner an, welcher ebenfalls postuliert, dass Kommunikationen die Elemente sozialer Systeme repräsentierten. Allerdings differiert TEUBNER dann hinsichtlich der Frage, wie die Autopoiesis sozialer Systeme zu konzipieren sei. Während bei LuHMANN Autopoiesis bereits eine notwendige Voraussetzung jedes sozialen Systems repräsentiert, gebraucht TEUBNER einen differenzierteren Ansatz. So folgt er in einem ersten Schritt zwar LuHMANN, dass

${ }^{398}$ Luhmann, N. (2008), S. 38. Vgl. auch Luhmann, N. (2008), S. 109-110; Luhmann, N. (1997), S. 19. Die grundsätzliche Frage, ob der Mensch als zentrales Systemelement eines sozialen Systems aufgefasst werden kann oder nicht, ist in der Literatur stark umstritten. Während einige Soziologen in der Tradition die Ausgliederung des Menschen aus dem sozialen System mit dem Hinweis auf die postulierte sonstige Hyperkomplexität des Systems verteidigen (vgl. z. B. Simon, F. B. (2017), S. 86) und anderen Rezipienten teilweise gar ein mangelndes Theorieverständnis vorwerfen (vgl. z. B. King, M. (2001)), existieren auch Gegenpositionen. So kritisiert beispielsweise MINGERS ,that there is a problem with the notion of production, specifically with the claim that it is communications (rather than people) that produce further communications. [...] Without human activity there would be no communication.“ (Mingers, J. (2002), S. 290, in der Quelle ebenfalls herv.). Hieraus folgt: „One communication may stimulate another, but surely it does not produce or generate it [...].“ (Mingers, J. (1995), S. 150, in der Quelle ebenfalls herv.). Vgl. auch die Anmerkungen bei VILLÁNYI, JUNGE und BROCK, welche hervorheben, dass ,,nur noch wenige bereit [seien], dahingehend zu folgen, dass ein soziales System ausschließlich aus Kommunikation besteht.“ (Villányi, D. / Junge, M. / Brock, D. (2009), S. 359). Auch HaBERMAS betrachtet die Systemtheorie LUHMANNS kritisch, stellt das kommunikativ handelnde Individuum der Lebenswelt in den Mittelpunkt und kritisiert deren „Kolonialisierung“ durch rein funktionalistische „Systeme“ (vgl. Habermas, J. (1997), S. 452), welche sich durch eine Institutionalisierung mittels Medium (z. B. Geld) auszeichnen (vgl. Brock, D. (2009), S. 175). Vgl. zur Abgrenzung der Konzepte von HABERMAS und LuHMANN auch Villányi, D. / Junge, M. / Brock, D. (2009), S. 359 sowie die Diskussion in Habermas, J. (1976). Schließlich kritisiert auch HAFERKAMP an LUHMANNS Konzeption den hohen Abstraktionsgrad, die hohe Komplexität sowie einen unüblichen Gebrauch von Prüfkriterien einer Konzeption. Vgl. Haferkamp, H. (1987), S. 51-58. 
Gesellschaftssysteme per se autopoietisch sind. Die Subsysteme der Gesellschaft jedoch müssen aus TEUBNERS Perspektive nicht notwendigerweise selbst autopoietisch $\operatorname{sein}^{399}$, sondern weisen zuerst einmal lediglich ein mehr oder minder großes Maß an Autonomie auf - womit TEUBNER explizit auf die Überlegungen VARELAS zurückgreift. ${ }^{400}$ TEUBNER differenziert in diesem Zusammenhang drei aufeinander aufbauende Stufen zunehmender Autonomie, welche er als Selbstbeobachtung, Selbstkonstitution und schließlich als Autopoiesis bezeichnet. ${ }^{401}$ Autopoiesis ist bei TEUBNER allerdings nicht auf die Reproduktion der Systemelemente eines sozialen Systems beschränkt, sondern wird in einem weiteren Schritt auch z. B. auf die systemkonstituierenden Prozesse sowie das Gesamtsystem ausgedehnt. In diesem Zusammenhang greift TEUBNER auf den wiederum ursprünglich aus der Biologie stammenden Begriff des Hyperzyklus zurück $^{402}$, welcher den auf einer Metaebene ${ }^{403}$ stattfindenden Prozess der Selbsterhaltung eines übergeordneten Systems beschreibt, der wiederum miteinander verknüpfte Prozesse der Selbstkonstitution umfasst, ,also die nochmalige zyklische Verkettung von zyklisch konstituierten Einheiten [...].“404 Soziale Systeme differenziert TEUBNER dann wiederum nach aufsteigender Autonomie in soziale Interaktionen, Gruppen sowie Organisationen, wobei letztere durch den postulierten Hyperzyklus mit dem zuvor aufgezeigten Autopoiesisbegriff korrespondieren. ${ }^{405}$ Insgesamt kann also festgehalten werden, dass die Konzeption TEUBNERS durch ein gradualistisches Verständnis des Autonomiekonzepts sozialer Einheiten gekennzeichnet ist, wobei der Grad der Ausprägung eine letztlich empirisch zu determinierende Frage darstellt. ${ }^{406}$

Betrachtet man nun sowohl das Werk LUHMANNS als auch HEJLS, so wäre die Frage nach einer sozialen Autopoiesis lediglich binär als existent oder

${ }^{399}$ Vgl. hierzu auch Teubner, G. / Nobles, R. / Schiff, D. (2002), S. 900; Teubner, G. (1992b), S. 613.

${ }^{400}$ Vgl. Teubner, G. (1987), S. 93.

${ }^{401}$ Vgl. Teubner, G. (1989), S. 44; Teubner, G. (1987), S. 101-102. Für eine noch differenziertere Klassifikation im Kontext der Selbstreferenz vgl. auch Teubner, G. (1989), S. 28-32.

402 Vgl. hierzu grundlegend Eigen, M. / Schuster, P. (1979), S. 2-6 sowie im Überblick Klipp, E. et al. (2016), S. 267-269; Leydesdorff, L. (2006), S. 184-190; Damiani, G. (2005), S. 257266.

${ }^{403}$ Vgl. Febbrajo, A. (1992), S. 24.

404 Teubner, G. (1987), S. 101. Vgl. auch Teubner, G. (1988), S. 218-220.

405 Vgl. Teubner, G. (1987), S. 113-117.

${ }^{406}$ Vgl. Teubner, G. (1987), S. 89-91 und S. 101-102. Zur Kompatibilität empirischer Forschung im Kontext autopoietischer Systeme vgl. auch Teubner, G. (1995), S. 142-147. 
nicht existent $\mathrm{zu}$ beantworten. ${ }^{407}$ Darüber hinaus existieren jedoch mit den Arbeiten von TEUBNER ${ }^{408}$ sowie von $\mathrm{KIRSCH}^{409}$ Ansätze, die eine soziale Autopoiesis im gradualistischen Sinne als denkbar erachten - eine Position, welche auch für diese Arbeit als fruchtbar aufgefasst wird. In diesem Sinne wären soziale Systeme graduell zwischen Allopoiesis und Autopoiesis zu verorten $^{410}$, wobei mit zunehmenden autopoietischen Eigenschaften das Ausmaß der Selbstbestimmtheit (Autonomie) des Systems ansteigt und Eigenschaften der Heteronomie bzw. Fremdbestimmtheit abnehmen. Diese Überlegung fasst nochmals die nachfolgende Abbildung 4.7 zusammen.

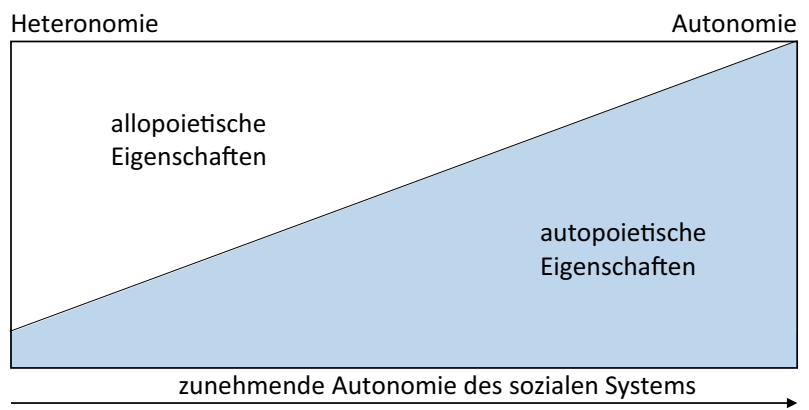

Abbildung 4.7 Graduelles Autopoiesis-Konzept sozialer Systeme

Soziale Systeme wären demnach nicht mehr binär als autopoietisch oder allopoietisch zu klassifizieren, sondern es existieren vielfältige Zwischenabstufungen zwischen beiden Extrema. Darüber hinaus folgt die Arbeit der Überlegung TEUBNERS hinsichtlich einer empirisch wahrscheinlichen Entwicklung autopoietischer Eigenschaften sozialer Systeme, wobei diese jedoch mit den Ausführungen KIRSCHS verbunden werden ${ }^{411}$, welcher den Menschen (und nicht Kommunikationen) als zentrales Element sozialer Systeme erachtet. Die Begründung

${ }^{407}$ So stellt LUHMANN fest: „Ein System setzt seine Reproduktion selbst fort oder es setzt sie nicht fort. Es gibt keine dazwischenliegenden Möglichkeiten." (Luhmann, N. (1985), S. 2). 408 Vgl. hierzu nochmals Teubner, G. (1987), S. 101-102.

${ }^{409}$ Vgl. Kirsch, W. (1997), S. 328-329; Kirsch, W. / Knyphausen, D. z. (1991), S. 94-96.

${ }^{410}$ Dabei sei auch festgehalten, dass soziale Systeme stets eine gewisse Eigendynamik aufweisen und daher niemals vollständig fremddeterminiert werden können, weshalb eine vollständige Heteronomie konzeptionell ausgeschlossen wird, wie auch Abbildung 4.7 aufzeigt. 411 Vgl. Lingnau, V. / Fuchs, F. (2019), S. 242. 
sozialer ${ }^{412}$ Systeme findet in diesem Kontext folglich durch die Interaktion von Individuen innerhalb dieser Systeme statt. In diesem Sinne sei davon ausgegangen, dass (menschliche) ${ }^{413}$ Individuen und deren (formalen wie informalen) Beziehungen soziale Systeme bilden, wobei gerade bei stabilisierten Systemen, wie dies (institutionelle) Organisationen repräsentieren ${ }^{414}$, Individuen bestimmte formalisierte Stellen ausfüllen und durch die stete Interaktion der jeweiligen Stelleninhaber empirisch erkennbar die organisationale Struktur aufrechterhalten, welches somit (unter bestimmten Rahmenbedingungen als Organisation) das Potential zur systemspezifischen Autopoiesis mitbegründet. Die vorausgegangenen Überlegungen fasst nochmals die nachfolgende Tabelle 4.1 zusammen.

Tabelle 4.1 Überblick über zentrale Positionen in der Autopoiesisdiskussion ${ }^{415}$

\begin{tabular}{|c|c|c|c|}
\hline \multicolumn{2}{|c|}{} & $\begin{array}{c}\text { Individuen als } \\
\text { Elemente }\end{array}$ & $\begin{array}{c}\text { Kommunikationen } \\
\text { als Elemente }\end{array}$ \\
\hline \multirow{2}{*}{ soutopoiesis } & ex definitione ja & Luhmann \\
\cline { 2 - 4 } & $\begin{array}{c}\text { empirischer } \\
\text { Grenzfall }\end{array}$ & $\begin{array}{c}\text { (Maturana) } \\
\text { Kirsch }\end{array}$ & Teubner \\
\cline { 2 - 4 } & $\begin{array}{c}\text { empirisch } \\
\text { wahrscheinlich }\end{array}$ & $\begin{array}{c}\text { eigene } \\
\text { Position }\end{array}$ & \\
\cline { 2 - 4 } & ex definitione nein & Hejl & \\
\hline
\end{tabular}

Diejenigen sozialen Systeme, welche nun auf der Mesoebene ein solches autopoietisches Potential und damit ein dauerhaftes, eigenes Zielsystem und genuine

${ }^{412}$ So betont auch der Begriff der Sozialität, dass diese Systeme wesentlich durch Ausrichtung von Handlungen am Verhalten der anderen Teilnehmer des Systems bestimmt werden. Vgl. in diesem Zusammenhang auch die klassische Definition bei Weber, M. (1976), S. 1.

${ }^{413}$ Dabei sei von weiteren Lebewesen (andere Hominide, aber auch z. B. Bienen, Ameisen etc.), welche Systeme bilden, die potentiell als sozial bezeichnet werden könnten, im Folgenden abstrahiert.

${ }^{414}$ Vgl. hierzu begrifflich auch detailliert den nachfolgenden Abschnitt 4.3.3.2.

415 In Anlehnung an Kirsch, W. (1997), S. 329. Dabei sei nochmals darauf hingewiesen, dass das genaue Begriffsverständnis der Autopoiesis zwischen den Konzeptionen durchaus variiert. Vgl. hierzu auch die Anmerkung bei Kirsch, W. (1997), S. 328. 
systemische Ansprüche besitzen ${ }^{416}$ - Organisationen im institutionellen Sinne -, werden im Folgenden detaillierter betrachtet.

\subsubsection{Zum Begriff der institutionellen Organisation}

Befasst man sich näher mit dem Terminus der Organisation, so zeigt sich recht schnell die im Detail vorherrschende Heterogenität der existierenden Begriffsauffassungen im sozialwissenschaftlichen Diskurs. So stellen in diesem Zusammenhang auch KUPER und THIEL pointiert fest: „Obwohl Organisation als ein Zentralbegriff der Sozialwissenschaften gelten kann, besteht kein Konsens über seine Definition. “417 Um diesen für die weiteren Ausführungen bedeutsamen Begriff konzeptionell präziser zu fassen, wird im Folgenden eine kurze Systematisierung vorgenommen und das für die weiteren Ausführungen maßgebliche Begriffsverständnis erarbeitet.

In einem ersten Schritt kann in Anlehnung an die klassische organisationswissenschaftliche Literatur zwischen einem instrumentellen und institutionellen Organisationsbegriff differenziert werden. ${ }^{418}$ Pointiert wird in diesem Zusammenhang vielfach festgestellt, dass erstere eine Organisation hat, während letztere eine Organisation ist. In diesem Sinne verweist der erste Begriff folglich auf eine Eigenschaft (Prädikat), welche Systemen im Sinne ihrer Geordnetheit bzw. Ordnung allgemein zugeschrieben wird. Der instrumentelle Organisationsbegriff beschreibt demnach, dass ein System organisiert ist. Hierauf aufbauend kann wiederum in einem zweiten Schritt zwischen der Aufbau- und Ablauforganisation differenziert werden. Während die Aufbauorganisation die Beziehungen bzw.

\footnotetext{
${ }^{416}$ So zeigen diese auch ein eigenes oberstes Ziel der Existenzsicherung, welches eine originär systemische Begründung der Relevanz von Unternehmensethik aus systemischem Eigeninteresse ermöglicht. Zur Abgrenzung der Begriffe „Ziel“" und „Anspruch“ vgl. auch die Ausführungen in Abschnitt 4.3.6.

${ }^{417}$ Kuper, H. / Thiel, F. (2018), S. 596. Ähnlich betont auch GRUNWALD: „Das Phänomen der Organisation wird in der Organisationslehre höchst unterschiedlich beschrieben und erklärt, je nachdem, welcher organisationstheoretische Ansatz zugrunde gelegt wird." (Grunwald, K. (2018), S. 223).

418 Vgl. z. B. Kräkel, M. (2015), S. 74-75; Marko, W. A. (2015), S. 294-296; Vahs, D. (2015), S. 15-17; Wolf, J. (2013), S. 46; Messi, N. / Pifko, C. (2010), S. 10; Weuster, A. (2010), S. 1; Oelsnitz, D. v. d. (2009), S. 18-22; Schwarz, S. (2009), S. 72; Hill, W. / Fehlbaum, R. / Ulrich, P. (1994), S. 17. Vgl. auch Macharzina, K. / Wolf, J. (2018), S. 474, wobei diese den funktionalen Organisationsbegriff synonym zum instrumentellen auffassen.
} 
Strukturen zwischen den Elementen widerspiegelt ${ }^{419}$, beschreibt die Ablauforganisation die stattfindenden Prozesse bzw. Interaktionen zwischen diesen Elementen und umfasst damit auch temporale Phänomene. ${ }^{420}$ Die genannten Teildimensionen können zudem noch weiter hinsichtlich der Zwecknotwendigkeit und Adaptivität über die Zeit hinweg unterschieden werden. So ist die Grundstruktur des Systems, wie bereits zuvor eruiert, notwendig zeitinvariant, während die Adaptions- bzw. Aufbaustruktur sich nach vorherrschenden Umweltbedingungen anpassen muss, um die Existenz des Systems zu sichern. Das System selbst jedoch, welches die aufgezeigte „Geordnetheit" sowie die innerhalb der Elemente stabilisiert homöostatisch stattfindenden Prozesse aufweist, wird in der Literatur häufig als institutionelle Organisation bezeichnet und korrespondiert damit am ehesten mit dem Alltagsverständnis des Terminus „Organisation“ als einem spezifischen komplexen $^{421}$ sozialen $^{422}$ System.

${ }^{419}$ Die Literatur differenziert in diesem Kontext auch oftmals weiter zwischen formaler (d. h. bewusster und kodifizierter) und informaler Organisation. Dieser Zusammenhang wird vielfach anhand des Eisbergmodells visualisiert, welches darauf hinweist, dass ein großer Teil der Strukturen implizit und nicht formalisiert ist und sich z. B. in unhinterfragten Annahmen bzw. kollektiven Basiswerten widerspiegelt. Vgl. Senior, B. / Swailes, S. / Carnall, C. (2020), S. 132; Macharzina, K. / Wolf, J. (2018), S. 480; Nicolai, C. (2018), S. 283-284; Jung, H. (2017), S. 418; Bergmann, R. / Garrecht, M. (2016), S. 7-8; Klimmer, M. (2016), S. 18-19; Schreyögg, G. (2016), S. 18-19; Kneubühl, D. (2015), S. 13; Paul, J. (2015), S. 286-291; Teuscher, H. (2011), S. 74; Deeg, J. / Küpers, W. / Weibler, J. (2010), S. 5257; Scherm, E. / Pietsch, G. (2007), S. 26-27; Bühner, R. (2004), S. 6-7; Göbel, E. (1998), S. 180; Kirsch, W. (1974), S. 64.

${ }^{420}$ Diese Differenzierung geht ursprünglich auf NORDSIECK und KosIOL zurück (vgl. Bea, F. X. / Göbel, E. (2019), S. 238-239; Oelsnitz, D. v. d. (2009), S. 108; Hansmann, K.-W. (2006), S. 199). Vgl. konzeptionell auch Thommen, J.-P. et al. (2020), S. 491-494; Bea, F. X. / Göbel, E. (2019), S. 238; Oehlrich, M. / Dahmen, A. (2019), S. 216; Hagenloch, T. (2018), S. 463; Macharzina, K. / Wolf, J. (2018), S. 479; Weber, W. / Kabst, R. / Baum, M. (2018), S. 131; Dillerup, R. / Stoi, R. (2016), S. 462; Hutzschenreuter, T. (2015), S. 436; Opresnik, M. O. / Rennhak, C. (2015), S. 48-55; Picot, A. et al. (2015), S. 28; Sigloch, J. / Egner, T. / Wildner, S. (2015), S. 90-99; Schlick, C. M. / Bruder, R. / Luczak, H. (2010), S. 455; Peters, S. / Brühl, R. / Stelling, J. N. (2005), S. 66-72; Eisenführ, F. / Theuvsen, L. (2004), S. 81; Hentze, J. / Heinecke, A. / Kammel, A. (2001), S. 151-152.

${ }^{421}$ In diesem Sinne bezeichnen auch SCHERER und MARTI Organisationen als ,hochkomplexe soziale Gebilde“ (Scherer, A. G. / Marti, E. (2019), S. 17).

${ }^{422}$ Es sei angemerkt, dass in der Literatur z. T. auch eine Erweiterung des institutionellen Organisationsbegriffs um technische Elemente zu einer „sozio-technischen Organisation“ vorgeschlagen wurde (so z. B. bei Jahnke, I. (2006), S. 37-41; Hentze, J. / Heinecke, A. / Kammel, A. (2001), S. 148). Ein solches Begriffsverständnis, welches auch als institutionelle Organisation i. w. S. aufgefasst werden könnte, ist jedoch insgesamt eher gering verbreitet. In diesem Sinne sei auch in dieser Arbeit die institutionelle Organisation i. e. S. in der Tradition 
Neben der aufgezeigten Zweiteilung hat die Literatur auch eine Fülle weiterer Differenzierungen erarbeitet ${ }^{423}$, welche terminologisch im Detail differieren. Betrachtet man allerdings die dort vorgestellten Systematiken näher, so zeigt sich, dass trotz aller Unterschiede begrifflich eine Gemeinsamkeit deutlich wird. So differenzieren viele Autoren neben dem aufgezeigten instrumentellen und institutionellen Begriffsverständnis drittens eine funktionale Perspektive auf den Organisationsbegriff, welcher auf die Funktion des Organisierens fokussiert und damit folglich eine der klassischen Managementfunktionen repräsentiert. ${ }^{424}$ So betonen auch KoONTZ, O'DONNELL und WeIHRICH neben Planning, Staffing, Leading und Controlling die Relevanz des Organizing als originären Bestandteil der allgemeinen Managementfunktion. ${ }^{425}$ Fasst man nun die bisherigen klassifikatorischen Überlegungen zusammen, so kann konstatiert werden: „Eine Organisation

eines besonderen sozialen Systems aufgefasst, welches sich z. B. in klassischen Definitionen wie einer ,purposeful social unit“ (Litterer, J. A. (1980), S. 2) oder „,social entities that [.] are goal-directed“" (Daft, R. L. (2016), S. 13) widerspiegelt. Paradigmatisch stellt in diesem Sinne auch MARTINEZ fest: ,The general textbook definition cites an organization as a collection of individuals engaged in interdependent, specialized activity in furtherance of a specific mission or goal [...]. In any case, the core insight is that organizations involve multiple individuals in pursuit of a common purpose." (Martinez, J. M. (2009), S. 57-58). Vgl. auch Aldrich, H. E. / Ruef, M. / Lippmann, S. (2020), S. 4.

${ }^{423}$ Vgl. auch die Übersicht bei Wolf, J. (2013), S. 45-46 sowie die Systematisierungen in Bea, F. X. / Göbel, E. (2019), S. 25-29; Schreyögg, G. / Geiger, D. (2016), S. 5-11; Fiedler, R. (2014), S. 1; Jones, G. R. / Bouncken, R. B. (2008), S. 27-28; Hansmann, K.-W. (2006), S. 191; Laux, H. / Liermann, F. (2005), S. 1-2; Schmidt, G. (2002), S. 5. Vgl. zudem die klassischen Abgrenzungen in Heinen, E. (1992), S. 49; Gutenberg, E. (1983), S. 235-236. Kosiol scheint aus heutiger Sicht die instrumentelle und institutionelle Perspektive miteinander zu verbinden. Dabei scheint er zu unterstellen, dass das, was als Resultat des Organisierens eine Organisation hat, auch eine Organisation ist. Vgl. hierzu Kosiol, E. (1978), S. 61-62.

${ }^{424}$ Vgl. Führer, A. et al. (2018), S. 12; Nicolai, C. (2018), S. 1-6; Bergmann, R. / Garrecht, M. (2016), S. 2-3; Dillerup, R. / Stoi, R. (2016), S. 462-463; Klimmer, M. (2016), S. 14-16; Kneubühl, D. (2015), S. 9; Picot, A. et al. (2015), S. 27; Straub, T. (2015), S. 405; Schulte-Zurhausen, M. (2014), S. 1-5; Jacob, M. (2013), S. 37; Nerdinger, F. W. (2012), S. 21-23; Siedenbiedel, G. (2010), S. 2-6; Bühner, R. (2004), S. 1-5; Hentze, J. / Heinecke, A. / Kammel, A. (2001), S. 148; Schuster, F. / Siemens, J. (1986), S. 1. Vgl. ähnlich auch Bea, F. X. / Göbel, E. (2019), S. 26-27; Grunwald, K. (2018), S. 224-225, welche relativ synonym den prozessorientierten Organisationsbegriff verwenden. Vgl. ähnlich auch Remer, A. / Hucke, P. (2007), S. 17-18 sowie Blum, E. (2000), S. 3; Mangler, W.-D. (2000), S. 17-22; Blum, E. (1991), S. 11, welche jedoch an Stelle des instrumentellen Organisationsbegriffs den strukturellen Organisationsbegriff gebrauchen.

${ }^{425}$ Vgl. Koontz, H. / O’Donnell, C. / Weihrich, H. (1984), S. 64-66. Dabei sei jedoch betont, dass der Begriff des Organizings über die (Selbst-)Organisation sozialer Systeme hinausgeht und auch die Fremdorganisation anderer Systeme, gerade im technischen Kontext, umfasst. 
(institutionell) hat eine Organisation (instrumentell) und bedarf der Organisation (funktional).“426 Die aufgezeigten Überlegungen fasst nochmals die nachfolgende Tabelle 4.2 zusammen.

Tabelle 4.2 Systematisierung des Organisationsbegriffs ${ }^{427}$

\begin{tabular}{|c|c|}
\hline Organisationsbegriff & Begriffsinhalt \\
\hline Organisation im funktionalen Sinne & Ein System wird organisiert \\
\hline Organisation im instrumentellen Sinne & $\begin{array}{r}\text { Ein System hat eine Organisation bzw. ist } \\
\text { organisiert }\end{array}$ \\
\hline Organisation im institutionellen Sinne & Ein System ist eine Organisation \\
\hline
\end{tabular}

Die aufgezeigte Begriffssystematik kann nun wiederum mit der bereits eruierten Differenzierung zwischen fremdorganisierten, d. h. allopoietischen, und selbstorganisierten, d. h. autopoietischen, Einheiten in Verbindung gebracht werden. So sind insbesondere autopoietische Systeme in der Lage, auch in komplexen Umwelten durch interne Selbstorganisation und -adaption ihre charakteristische Grundstruktur zu erhalten. Der funktionale Organisationsbegriff ist folglich diesen Systemen inhärent und ermöglicht diesen, ihre Organisation im instrumentellen Sinne in Bezug auf die Grundstruktur zu erhalten bzw. ihre weiteren Aufbaustrukturen an sich wandelnde Umwelterfordernisse $\mathrm{zu}$ adaptieren. Als institutionelle Organisationen können nun genau diejenigen Entitäten aufgefasst werden, welche durch funktionale Organisation im Stande sind, ihre Existenz (bzw. Organisiertheit) auf Dauer aufrechtzuerhalten. ${ }^{428}$ Bei diesen sind die drei aufgezeigten

${ }^{426}$ Lingnau, V. / Beham, F. / Fuchs, F. (2020), S. 23, in der Quelle ebenfalls herv. Vgl. ähnlich auch Führer, A. et al. (2018), S. 12; Kneubühl, D. (2015), S. 1; Schuster, F. / Siemens, J. (1986), S. 1.

${ }^{427}$ Lingnau, V. / Beham, F. / Fuchs, F. (2020), S. 23.

${ }^{428}$ Sie sind somit in der Lage, sich selbst Institutionen im Sinne gemeinsamer Regeln zu geben. Vgl. hierzu auch den klassischen Institutionsbegriff bei North, D. C. (2011), S. 3. Solche Entitäten könnten mit APEL auch als „Selbstbehauptungssysteme“ (Apel, K.-O. (1992), S. 61, in der Quelle ebenfalls herv.) bezeichnet werden. Eine Analogie zu klassischen biologischen Überlegungen findet sich in diesem Zusammenhang z. B. bei PADGETT und POWELL, welche feststellen: „In organisms, social or biological, rules of action and patterns of interaction persist and reproduce in the face of continual turnover in component parts, be these cells, molecules, principals, or agents. In the flow of people through organizations, the collectivity is not renegotiated anew." (Padgett, J. F. / Powell, W. W. (2012), S. 8). 
Begriffe folglich so miteinander verknüpft, dass die institutionelle Organisation durch die Fähigkeit der funktionalen Organisation ihre instrumentelle Organisation im Kern erhalten und im Detail adaptieren kann, um ihre Existenz zu sichern. Die Möglichkeit der Entstehung autopoietischer Eigenschaften als empirisches Phänomen kann auch im organisationalen Kontext durch den Begriff der Emergenz erklärt werden ${ }^{429}$, welcher zuerst im Rahmen philosophischer Arbeiten von LEWES über die Entstehung des menschlichen Geistes geprägt ${ }^{430}$ und mittlerweile in einer Vielzahl von Wissenschaftsdomänen aufgenommen wurde. ${ }^{431}$ Grundgedanke des Emergenzbegriffs ist dabei, dass im systemischen Kontext neue Eigenschaften spontan entstehen können, welche nicht in den konstituierenden Elementen bereits enthalten sein müssen bzw. nicht auf deren Summe trivial zu reduzieren wären. ${ }^{432}$ So stellt beispielsweise HARTIG-PERSCHKE fest:

${ }^{429}$ In diesem Sinne erklärt auch OLSEN die Entstehung von (institutionellen) Organisationen bzw. sozialer Organisiertheit (vgl. hierzu auch Olsen, M. E. (1978), S. 6) durch Emergenz: „Social organization develops as individuals interact to create ongoing relationships that form patterns of social ordering that become infused with shared meanings. That is, organization emerges from the actions of individual personalities and can never be completely separated from them." (Olsen, M. E. (1978), S. 8). Dabei hebt OLSEN aber auch den dynamischen Charakter von Organisationen hervor: "If we look only at the static structure of an organization - such as portrayed by a formal organizational chart - we shall miss entirely the process through which the organization is constantly emerging from the actions of its members.“ (Olsen, M. E. (1978), S. 10). Verallgemeinert konstatiert auch CoRNING: „Emergence is what ,self-organizing' processes produce." (Corning, P. A. (2002), S. 18).

${ }^{430}$ LEWES beschäftigte sich dabei mit der Frage, wie aus materiellen Strukturen des Gehirns die Entstehung des Geistes als neue „Qualität“ bzw. Eigenschaft gedacht werden könnte. In diesem Sinne formulierte er den zentralen Inhalt des Emergenzgedankens wie folgt: „The emergent is unlike its components in so far as these are incommensurable, and it cannot be reduced either to their sum or their difference." (Lewes, G. H. (1875), S. 413). Vgl. auch Friedrich, A. (2015), S. 323; Goldstein, J. (2011), S. 66; Goldspink, C. / Kay, R. (2010), S. 49; Sedlacek, K.-D. (2010), S. 44; Hartig-Perschke, R. (2009), S. 48; Skirl, H. (2009), S. 15; De Wolf, T. / Holvoet, T. (2005), S. 2; Stephan, A. (2000), S. 33; Goldstein, J. (1999), S. 53; Emmeche, C. / Køppe, S. / Stjernfelt, F. (1997), S. 85. Für eine noch tiefere historische Analyse vgl. Stephan, A. (2016), S. 75-77; Bedau, M. A. / Humphreys, P. (2008), S. 11; Kim, J. (2008), S. 127-128.

${ }^{431}$ Vgl. Richling, J. et al. (2011), S. 47; Sedlacek, K.-D. (2010), S. 45; Skirl, H. (2009), S. 14-41.

${ }^{432}$ Vgl. Sedlacek, K.-D. (2010), S. 44; Wolf, G. (2010), S. 34; Skirl, H. (2009), S. 16; Güldenberg, S. (2003), S. 104; Völcker, W. (1998), S. 13. So stellt in diesem Zusammenhang auch WILlKE fest: „Der Kern des Systemischen ist Emergenz.“ (Willke, H. (2018), S. 1617). Ebenso kommt auch EsTEVÃo zum Schluss, dass „Publikationen zur Emergenz meist in den Kontext systemischen Denkens eingebettet sind [...].“ (Estevão, M.-J. (2011), S. 35). Zum Überblick über die Verbreitung in verschiedenen Wissenschaftsdiziplinen vgl. ebenso 
„Mit dem Terminus ,Emergenz“ wird üblicher Weise das Auftreten neuer Eigenschaften auf einer ,höheren ' Ordnungs- oder Systemebene bezeichnet. “433 Ebenso konstatieren auch KROHN und KUPPERS paradigmatisch: „Emergenz bezeichnet das plötzliche Auftreten einer neuen Qualität, die jeweils nicht erklärt werden kann durch die Eigenschaften oder Relationen der beteiligten Elemente, sondern durch eine jeweils besondere selbstorganisierende Prozeßdynamik [...]. "434 Ähnlich subsumiert GOLDSTEIN: „Therefore, explanations that include the construct of emergence contain the claim that emergent phenomena are neither predictable from, deducible from, nor reducible to the parts alone. ${ }^{6435}$ Der Emergenzbegriff ist damit paradigmatisch prinzipiell antireduktionistisch geprägt. ${ }^{436}$ In der Literatur wird in diesem Zusammenhang noch häufig zwischen starker und schwacher Emergenz differenziert, wobei bei ersterer die emergenten Eigenschaften grundsätzlich und bei letzterer gegenwärtig unter Nutzung aktuell verfügbaren Wissens

Estevão, M.-J. (2011), S. 17-34 sowie zur Entstehungsgeschichte des Begriffs auch De Wolf, T. / Holvoet, T. (2005), S. 2-3; Sawyer, R. K. (2005), S. 21-45; Corning, P. A. (2002), S. 18-21; Stephan, A. (2000), S. 33-35; Goldstein, J. (1999), S. 51-57; Völcker, W. (1998), S. 12-27; Stephan, A. (1992), S. 25-45.

${ }^{433}$ Hartig-Perschke, R. (2009), S. 44.

${ }^{434}$ Krohn, W. / Küppers, G. (1992), S. 7-8, in der Quelle ebenfalls herv.

435 Goldstein, J. (1999), S. 57. Auch TeUbNER stellt in diesem Sinne fest: „Gemeinhin benutzt man den Begriff Emergenz, um das Auftreten von etwas Neuem im Evolutionsprozeß zu kennzeichnen oder um auszudrücken, daß das Ganze mehr ist als die Summe seiner Teile [...].“ (Teubner, G. (1992a), S. 191). Ebenso konstatiert auch TELLER: „1) Emergent properties are in some sense, novel'. And 2) an emergent property of a whole cannot be predicted from knowledge of the properties of the parts." (Teller, P. (1992), S. 139). Analog definiert WILLKE: „Emergent soll eine Ordnung oder eine Eigenschaft heißen, wenn sie aus der bloßen Aggregation von Teilen oder aus den summierten Eigenschaften der Teile nicht mehr erklärbar ist.“ (Willke, H. (2018), S. 17, in der Quelle ebenfalls herv.). Ähnlich resümiert STEPHAN: „Häufig dient [der Emergenzbegriff] zur Charakterisierung solcher Systemeigenschaften, die sich nicht auf die eine oder andere Weise auf die Eigenschaften der Systembestandteile zurückführen lassen oder die unvorhersagbar sind oder gar einen ,abwärts gerichteten“ kausalen Einfluß auf die Systemkomponenten ausüben." (Stephan, A. (2016), S. XI).

${ }^{436}$ So stellt auch HOYNINGEN-HUENE fest: „Der Emergenzbegriff markiert, so verstanden, einen Gegensatz zum Reduktionismus: Etwas, das emergent ist, kann gerade nicht auf die tiefere Ebene reduziert werden." (Hoyningen-Huene, P. (1994), S. 324). Vgl. auch Greve, J. / Schnabel, A. (2014), S. 10; Sedlacek, K.-D. (2010), S. 46; Hartig-Perschke, R. (2009), S. 44; Bedau, M. A. / Humphreys, P. (2008), S. 10; Crutchfield, J. P. (2008), S. 269; Luisi, P. L. (2002), S. 46. 
und Methoden nicht erklärt werden können. ${ }^{437}$ Dabei sei es für die weiteren Überlegungen aus pragmatischer Perspektive als ausreichend erachtet, dass die „,neue Qualität“ gegenwärtig nicht erklärt werden kann, unbeschadet der Frage, ob dies nur einem aktuell fehlenden methodischen oder faktenbezogenen Wissens geschuldet oder prinzipiell unmöglich ist. ${ }^{438}$

Der Begriff der Emergenz ist wiederum eng mit dem Konzept der Autopoiesis verknüpft, wie auch HARTIG-PERSCHKE hervorhebt: „Tatsächlich stehen sich der Begriff ,Autopoiesis“ und der Begriff der ,Emergenz“ also recht nahe.“439 Ähnlich konstatiert auch BLANCHARD, dass der Begriff der Emergenz „oft in Verbindung mit dem Wort ,Selbstorganisation“ gebraucht [wird].“440 Als grundlegende Voraussetzung von Emergenz gilt dabei das Vorhandensein einer ausreichenden Komplexität. So stellt auch STEPANIC fest: „The notions of complex systems and emergence are rather closedly related.“441 Ähnlich resümiert auch SAWYER: „[T]here is a consensus that complex systems may have autonomous laws and properties at the global level that cannot be easily reduced to lower-level, more basic sciences." 442 Dabei gilt jedoch, dass auch andere Systeme komplex sein können, jedoch nicht notwendigerweise Kerneigenschaften der Autopoiesis bzw. Lebensfähigkeit entwickeln müssen. ${ }^{443}$ So kommt es

${ }^{437}$ Vgl. Greve, J. (2019), S. 203; Roth, G. / Strüber, N. (2019), S. 273; Greve, J. (2015), S. 11-12; Bedau, M. A. (2014), S. 66-69; Greve, J. / Schnabel, A. (2014), S. 10-12; Sedlacek, K.-D. (2010), S. 44-45; Hoyningen-Huene, P. (2009), S. 193; Chalmers, D. J. (2006), S. 244-245; Bedau, M. A. (1997), S. 376-379; Hoyningen-Huene, P. (1994), S. 324. Vgl. ähnlich auch Luisi, P. L. (2002), S. 48; Goldstein, J. (1999), S. 59 sowie Stephan, A. (2016), S. 66-72; Hartig-Perschke, R. (2009), S. 50-56; Stephan, A. (2000), S. 35-45, welche neben der schwachen Emergenz auch die synchrone und diachrone Emergenz differenzieren. Vgl. hierzu auch Sartenaer, O. (2015), S. 34-35 sowie den Überblick bei Richling, J. et al. (2011), S. 48.

${ }^{438}$ Darüber hinaus wäre aus wissenschaftstheoretischer Perspektive auch die Frage zu stellen, inwiefern eine solche prinzipielle Nichterklärbarkeit überhaupt im strengen Sinne gezeigt oder zumindest plausibilisiert werden kann. So stellt z. B. auch VANBERG im soziologischen Kontext fest: „Die ,Reduzierbarkeit“ von Soziologie auf Psychologie ist eine Frage des faktisch gegebenen Theoriestandes; ein ,prinzipielles‘ Argument gegen die Möglichkeit einer solchen Reduktion gibt es nicht." (Vanberg, V. (1975), S. 261, in der Quelle ebenfalls herv.).

${ }^{439}$ Hartig-Perschke, R. (2009), S. 104.

${ }^{440}$ Blanchard, P. (2014), S. 111. Vergleichbar stellt auch NKOYO fest, dass ,der Begriff der Selbstorganisation dem der Emergenz [ähnele].“ (Nkoyo, E. I. (2016), S. 185-186).

441 Stepanić, J. (2010), S. 199.

442 Sawyer, R. K. (2005), S. 4. Vgl. hierzu auch Walby, S. (2007), S. 462.

443 So kann in komplexen Systemen auch Chaos herrschen und diese keine eigenständige Strukturbildung aufweisen. Es kommt im Zusammenhang der Lebensfähigkeit folglich auf 
bei Lebewesen evident nicht nur auf Komplexität als alleinige Voraussetzung der Lebensfähigkeit an, sondern vielmehr muss innerhalb der Komplexität auch eine bestimmte Konfiguration bzw. Geordnetheit der Grundstruktur schon ex ante vorliegen, welche das entstehende System überhaupt in die Lage versetzt, seine Homöostase zu initiieren, sich folglich stabil gegenüber seiner Umwelt abgrenzen zu können. Bei institutionellen Organisationen findet sich diese Initialstruktur insbesondere in der formalen Organisation, welche die Entstehung strukturierter Selbsterhaltungsprozesse im Rahmen arbeitsteilig organisierter Tätigkeiten begünstigt bzw. als dessen Katalysator erachtet werden kann. Dies erscheint gerade auch vor dem Hintergrund plausibel, dass organisationale Stellen teilweise (gerade im rechtlichen Kontext) als „Organe“ bezeichnet werden. Die vorausgegangenen Überlegungen zeigen damit, dass die Komplexität eines sozialen Systems demnach als notwendige Voraussetzung zur Entstehung autopoietischer Systemeigenschaften aufgefasst werden kann. Hierzu muss jedoch ein hinreichendes $\mathrm{Ma} \beta$ an initialer Struktur hinzutreten, um autopoietische Prozesse zu ermöglichen.

Institutionelle Organisationen sind vor diesem Hintergrund dementsprechend in der Lage, irreduzible systemische Eigenschaften zu entwickeln, welche sich nicht notwendigerweise auf die isolierten Eigenschaften ihrer Mitglieder ${ }^{444}$ zurückführen lassen und diese folglich übersteigen. Während also einfache Sozialsysteme, wie diese sich in alltäglichen Interaktionen zeigen, nur ein geringes Maß an autopoietischen Eigenschaften und damit nur ein geringes Maß an Autonomie aufweisen, können institutionelle Organisationen emergent Eigenschaften der Lebensfähigkeit entwickeln, welche sich nach innen durch kollektive Identität ${ }^{445}$,

eine schon zu Beginn existierende Ordnung des Systems an, welche diesem unter Nutzung seiner Komplexität das Potential verschafft, Eigenschaften des Lebendigen zu entwickeln.

444 So stellt auch FETZER fest: „Organisationen haben Mitglieder und Nicht-Mitglieder. Durch diesen spezifischen Abgrenzungsmechanismus unterscheiden sie sich nicht nur von Funktionssystemen, sondern auch von zufälligen Menschenansammlungen oder ähnlichem.“ (Fetzer, J. (2004), S. 69). Hinsichtlich der genauen Umfangsbestimmung institutioneller Organisationen hat die Literatur jedoch eine Fülle an Ansätzen hervorgebracht. Im Weiteren soll auch hier wiederum den Überlegungen nach LINGNAU, BEHAM und FUCHS gefolgt werden, nach denen sich der organisationale Umfang anhand der Weisungsbefugnisse bzw. -gebundenheit sowie der Mitentscheidungs- und Kontrollbefugnisse bestimmt, welche die organisationale Grenze konstituieren. Vgl. hierzu grundlegend Lingnau, V. / Beham, F. / Fuchs, F. (2022). In diesem Sinne werden dann , die Einheiten zu Komponenten, bilden damit eine autonomisierte und selektive Organisation und somit das System, zu dem sie gehören und das auf sie wirkt." (Hejl, P. M. (1992b), S. 280).

445 In diesem Sinne stellt auch GOLDSTEIN fest: ,[E]mergents appear as integrated wholes that tend to maintain some sense of identity over time." (Goldstein, J. (1999), S. 50). 
geteilte Werte sowie überindividuelle (organisationale) Ziele bzw. Ansprüche ${ }^{446}$ und nach außen durch Selbsterhalt und gemeinsames Auftreten gegenüber der Umwelt manifestieren. Durch dieses autopoietische Potential können (institutionelle) Organisationen auch als quasi-lebende Systeme definiert werden, welche ihre eigene Existenz auf Dauer sichern und sich somit von weniger komplexen, nicht-dauerhaften sozialen Zusammenkünften des Alltags wie einfachen, kurzlebigen Interaktionssystemen abgrenzen lassen. Diese Überlegungen zeigt nochmals die nachfolgende Abbildung 4.8 auf.

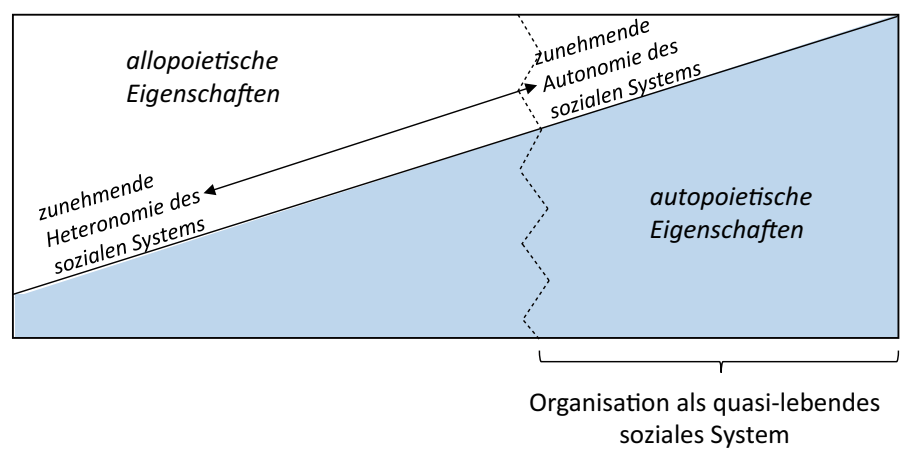

Abbildung 4.8 Institutionelle Organisationen als spezifische soziale Systeme

Basierend auf diesen Überlegungen können Organisationen im institutionellen Sinne nun wie folgt definiert werden: ,(Institutionelle) Organisationen sind spezifische zeitstabile soziale Systeme, welche emergent-autopoietische Eigenschaften entwickeln und damit ihre Grundstruktur dauerhaft selbst aufrechterhalten können. ${ }^{447}$ Sie werden durch von Menschen ausgefüllte Stellen (Organisationsmitglieder) und deren (formalen wie informalen) Beziehungen gebildet. Die auf Dauer ausgerichtete Grundstruktur ist dabei für die Elemente und deren Beziehung bindend (z. B. durch gemeinsame Zweckbindung). “448

${ }^{446}$ Zur Abgrenzung der Begriffe „Ziele“ und „Ansprüche“ vgl. die Ausführungen in Abschnitt 4.3.6.

${ }^{447}$ In diesem Sinne konstatieren auch KATZ und KAHN: „All social systems, including organizations, consist of patterned activities of a number of individuals. [...] [T] hey are repeated, relatively enduring, and bounded in space and time. If the activity pattern occurs only once or at unpredictable intervals, we could not speak of an organization." (Katz, D. / Kahn, R. L. (1978), S. 20).

${ }^{448}$ Lingnau, V. / Beham, F. / Fuchs, F. (2020), S. 24, in der Quelle ebenfalls herv. 
In diesem Zusammenhang erscheint vor dem Kontext der organisationalen Autopoiesis abschließend noch eine kurze Diskussion des Reproduktionsbegriffs relevant. So kritisiert ein Teil der Literatur den Transfer des Autopoiesisbegriffs auf soziale Systeme, welche aus Individuen bestehend konzipiert werden ${ }^{449}$, da in Organisationen schließlich keine Individuen „produziert“ würden. ${ }^{450}$ Eine solche, sicherlich teilweise ironisch konnotierte, Feststellung greift jedoch aus mehreren Gründen zu kurz. Dabei scheinen zuerst einmal die pauschale Kritik sowie vielfältige Missverständnisse in diesem Zusammenhang häufig darauf zu beruhen, dass menschliche Individuen ausschließlich als biochemische Einheiten aufgefasst werden, welches jedoch aus ethischer, aber auch anthropologischer Perspektive in kritischer Weise reduktionistisch erscheint und als Basiswerturteil eigentlich begründungspflichtig wäre - allerdings interessanterweise im einschlägigen Schrifttum kaum thematisiert wird. Vielmehr ist doch gerade in Umkehrung dieser Auffassung ersichtlich, dass eine Annahme von Aufgaben bzw. formalisiert - von organisationalen Stellen - als Mitglied nicht nur eine überindividuelle oder soziale Konstruktion bzw. ein „Label“ darstellt, sondern (gerade im Laufe der Zeit der Mitgliedschaft) auch Teil der Identität, Fähigkeiten und des Wesens eines Menschen wird, welches mit dem organisationalen System fortgeschrieben wird. Man nimmt eben nicht nur die Rolle des Vereinsvorstands oder Hochschullehrers ein, sondern ist dies auch. ${ }^{451}$ Darüber hinaus, und hierauf weist bereits TEUBNER hin, greift zweitens eine Reduktion des Autopoiesisbegriffs auf die Erzeugung der Elemente zu kurz, weshalb er eine Erweiterung auf Prozesse, Metaprozesse sowie das ganze System vorschlägt. ${ }^{452}$ Besonders letztere Idee erscheint hier konzeptionell bedeutsam. So bezieht sich die Autopoiesis nicht nur auf den Erhalt sozialer Systemelemente - hier also das Individuum in seiner Stelle - sondern vielmehr gerade auf das gesamte System, welches sich gegenüber seiner Umwelt selbst zu erhalten vermag. Dabei ist im strengen Sinne die vorige, von TEUBNER aufgezeigte Begriffsreihung redundant, da der Erhalt des

\footnotetext{
449 So z. B. Teubner, G. (1987), S. 92; Varela, F. J. (1981), S. 15.

${ }^{450}$ Eine Ausnahme könnten jedoch Familien oder auch Gesellschaften darstellen, da in diesem Kontext auch eine „Reproduktion“ von Individuen durch Geburten als konzeptionell sinnvoll erachtet werden könnte. In Anlehnung an die Bezeichnung der institutionellen Organisation als quasi-lebende Einheit könnte damit im organisationalen Kontext auch von einer „Quasireproduktion“ im Sinne der natürlichen Autopoiesis gesprochen werden.

${ }^{451}$ Es sei daher nochmals darauf hingewiesen, dass die Elemente des sozialen Systems nicht ,,nur“ Individuen im biochemischen Sinne sind, sondern Individuen als Systemmitglieder, deren Mitgliedschaft im System durch stetige Interaktion fortgeschrieben wird. Soziale Systeme benötigen als Elemente beides: das Individuum und seine Funktion.

452 Vgl. Teubner, G. (1989), S. 44; Teubner, G. (1987), S. 101.
} 
Systems als Ganzes freilich schon die konstituierenden systemischen Prozesse wie auch dessen Subsysteme bzw. Elemente inkludiert. Verzichtet man folglich auf eine verkürzte biochemische Begriffskonnotation im Kontext einer Elementeproduktion und fokussiert vielmehr auf das Systemganze, so ist ein Transfer auf die soziale Ebene weit weniger problematisch. Zum Kern des Autopoiesisbegriffs soll in diesem Sinne also die gesamtsystemische Selbsterhaltung gewählt werden, welches dann wiederum auch Prozesse der Selbstorganisation sowie eine kollektive Identität des Systems umfasst, auf welche diese Prozesse bezogen sind. So besitzen Organisationen (aus sich selbst heraus!) gegenüber ihrer Umwelt das Potential, ihre Existenz zu erhalten, indem die Strukturen des Systems intern adaptiert werden ${ }^{453}$, welches sich z. B. in einem Diskurs über die Änderung von Geschäftspraktiken oder die Schaffung neuer Stellen manifestiert. Abschließend kann darüber hinaus auch noch aus einer weiteren Perspektive der teils immaterielle Substanzcharakter der Organisation als Einheit verdeutlicht werden. Hierbei nimmt zur Erhaltung des organisationalen Ganzen - insbesondere vor dem Hintergrund der fortschreitenden Digitalen Transformation - der Begriff des Wissens eine zentrale Rolle ein. Betrachtet man diesen genauer im organisationalen Kontext, so zeigt sich auch hier, dass die organisationale Autopoiesis nicht auf die Reproduktion von physischen Entitäten abzielt. Es kann somit konstatiert werden: „Der (Re-)Produktion innerhalb einer Organisation liegen [.] keine (bio-) chemischen Prozesse zugrunde, sondern Prozesse von Aufnahme, Verarbeitung und Weitergabe von Informationen bzw. Wissen ${ }^{454}$ im Rahmen der organisationalen Beziehungen [...]. “455

Die Bedeutung des Wissens lässt sich im organisationalen Kontext mit einigen klassischen Überlegungen aus der Managementliteratur verknüpfen. So können auch im Sinne GARUDS und PORACS Manager als ,information workers“456

${ }^{453}$ So konstatiert auch COLEMAN: „Complexity theory views organizations as ,complex adaptive systems" that coevolve with the environment through the selforganizing behavior [...].“ (Coleman, H. J. (1999), S. 33).

${ }^{454}$ Siehe hierzu auch die Überlegungen in Abschnitt 4.6 sowie zum Wissensbegriff Abschnitt 4.2.4.2.

455 Lingnau, V. / Beham, F. / Fuchs, F. (2020), S. 25, in der Quelle ebenfalls herv.

${ }^{456}$ Lingnau, V. (2006), S. 1, in der Quelle fett herv. So betonen GARUD und PorAC: ,[M]anagers [.] are confronted with the task of making sense of very complex and ambiguous environments." (Garud, R. / Porac, J. F. (1999), S. XIV). 
aufgefasst werden. Ähnlich subsumieren SCHERMERHORN und BACHRACH: „Managers are information processors who use analytical, technological, and information competencies to fulfill their roles in the management process. “" ${ }^{\circ 57}$ Folglich ist das Vorhandensein von organisationalem Wissen entscheidend, um fundierte Entscheidungen zu treffen, welches im Weiteren der Arbeit auch nochmals im Kontext des strukturierten Legitimitätsmanagements aufgegriffen wird. Dabei spielen aber ebenso, wie gerade das Zitat von SCHERMERHORN und BACHRACH aufzeigt, zunehmend technologische Elemente eine zentrale Rolle, so dass im Folgenden noch kompakt auf den Komplementärbegriff des technischen Systems eingegangen wird, bevor schließlich beide im Gesamtmodell des Unternehmens als sozio-technisches System integriert werden.

\subsubsection{Das technische bzw. IMF-System in der Wissensgesellschaft}

Der Begriff des technischen Systems wird sowohl fachspezifisch im Rahmen der betriebswirtschaftlichen Forschung als auch darüber hinaus in einer Vielzahl von Begriffskonnotationen verwendet. So findet sich beispielsweise gerade im ingenieurwissenschaftlichen Kontext häufig eine Klassifikation, welche zwischen materialbasierten, energiebasierten und informationsbasierten technischen Systemen differenziert. ${ }^{458}$ In der betriebswirtschaftlichen Literatur zeigt sich dagegen häufig eine güterspezifische Abgrenzung ${ }^{459}$, welche auch menschliche Individuen als Produktionsfaktor bzw. Ressource auffasst. ${ }^{460}$ Einer solchen, obschon verbreiteten Begriffsauffassung soll im Weiteren allerdings nicht unkritisch gefolgt werden. So würde zum einen eine undifferenzierte Inklusion die inhaltlich bedeutsame Differenzierung zwischen sozialen und technischen Systemen vernachlässigen, zum anderen erscheint die terminologisch gleichlautende

${ }^{457}$ Schermerhorn, J. R. / Bachrach, D. G. (2020), S. 137. Zum Begriff und zur Bedeutung des Managements vgl. auch die detaillierten Ausführungen in Abschnitt 4.4.1.

458 Vgl. z. B. Czichos, H. (2019b), S. 9; Conrad, K.-J. (2015), S. 27; Czichos, H. (2015), S. 13; Reuter, M. (2014), S. 46-47; Walter, H. (2009), S. 49; Johannsen, G. (1993), S. 19-22. Vgl. hierzu auch den historischen Überblick bei Schindler, C. (2009), S. 34-37.

${ }^{459}$ Vgl. Thommen, J.-P. et al. (2020), S. 4-5; Weber, W. / Kabst, R. / Baum, M. (2018), S. 23; Vahs, D. / Schäfer-Kunz, J. (2015), S. 9-13 sowie klassisch Heinen, E. (1992), S. 128-129. ${ }^{460}$ Der Begriff wird dabei besonders häufig im Rahmen des Human Resource Managements gebraucht. Vgl. z. B. Schellinger, J. et al. (2020), S. 185; Miebach, B. (2017), S. 29; Rowold, J. (2015), S. VII. 
Einordnung von technischen Systemen und Menschen als „Gut“ selbst problematisch, wie auch die Wahl des Terminus „Humankapital“ zum Unwort des Jahres 2004 verdeutlicht. ${ }^{461}$ Wird nun aber im betriebswirtschaftlichen Diskurs genauer zwischen sozialen und technischen Systemen differenziert, so zeigt sich, dass unter der Bezeichnung des technischen Systems vielfach primär die Betriebsmittel (und ggf. materielle Ressourcen) subsumiert werden, welches konzeptionell jedoch noch zu kurz greift. Aus diesem Grund sei im Folgenden ein erweitertes technisches Systemmodell vorgestellt, welches in Anlehnung an die Vorschriften des deutschen Handelsgesetzbuches das technische System in drei Subsysteme differenziert. ${ }^{462}$ In diesem Sinne kann das technische System nochmals in ein immaterielles, materielles sowie ein finanzielles Teilsystem untergliedert werden, wobei das Gesamtsystem hieran angelehnt als IMF-System bezeichnet wird. Das immaterielle Subsystem umfasst dabei alle nichtkörperlichen Gegenstände, wie z. B. Patente, Lizenzen, Markenrechte, Software usw., aber auch Wissen, welches im Zuge der Wissensgesellschaft, wie aufgezeigt wurde, zunehmend an Relevanz für einen dauerhaften unternehmerischen Erfolg gewinnt. Dagegen beinhaltet das materielle Subsystem alle physischen Güter wie die klassischen Betriebsmittel, d. h. Maschinen und Anlagen, aber auch betriebliche Grundstücke sowie Vor-, Teil- und Fertigprodukte, welche sich durch ihre materielle Substanz auszeichnen. Darüber hinaus können dem materiellen Subsystem auch die betrieblich genutzten Pflanzen und Tiere zugerechnet werden. Neben diesen beiden realgüterlichen Subsystemen kann im Rahmen der IMF-Klassifikation schließlich noch das Finanzsystem des Unternehmens als eigenständiges System herausgestellt werden, welches die monetären Mittel (Nominalgüter) enthält.

Die genaue Zusammensetzung bzw. relative Bedeutung der drei Subsysteme basiert dabei wiederum auf dem genauen Geschäftsmodell eines spezifischen Unternehmens. So ist ersichtlich, dass bspw. bei Finanzdienstleistern wie Banken oder Versicherungen, bei welchen die Wertschöpfungstätigkeit wesentlich durch den Einsatz finanzieller Mittel geprägt ist, das Finanzsystem im Fokus der unternehmerischen Tätigkeit liegt, während z. B. wiederum die existierenden materiellen Systeme, wie die IT-Infrastruktur, in der Regel eine untergeordnete bzw. sekundäre Rolle einnehmen. Bei Softwaredienstleistern dürfte dagegen regelmäßig ein stärkerer Fokus auf dem immateriellen System (Softwareprodukte bzw. Dienstleistungen) liegen, während wiederum bei klassischen Industrieunternehmen meist das materielle System von zentraler Bedeutung ist. Insgesamt gilt

\footnotetext{
461 Vgl. Miebach, B. (2014), S. 197; Marcus, B. (2011), S. 15.

462 So wird in diesem Zusammenhang u. a. zwischen materiellen, immateriellen und finanziellen Aktiva differenziert. Vgl. hierzu § 266 Abs. 2 HGB (2021).
} 
jedoch, dass die drei Subsysteme keine isolierten Einheiten darstellen, sondern vielmehr durch vielfältige Interaktionen sowie Interdependenzen gekennzeichnet sind. ${ }^{463}$ Im Rahmen der Wertschöpfungstätigkeit sind neben den internen, d. h. organisationszugehörigen Stakeholdern, welche auch für die Steuerung des technischen Systems Verantwortung übernehmen, vielfältige externe Stakeholder involviert, die z. B. als Lieferanten materielle Realgüterströme oder als Kapitalgeber Nominalgüterströme in das technische System einbringen, bzw. als Konsumenten den unternehmerischen Output entgegennehmen, welches dann wiederum mit einem Zahlungsinput in das Finanzsystem einhergeht. Die aufgezeigten Überlegungen zur Konzeption des technischen Systems eines Unternehmens als IMF-Systems fasst nochmals kompakt die nachfolgende Abbildung 4.9 zusammen.

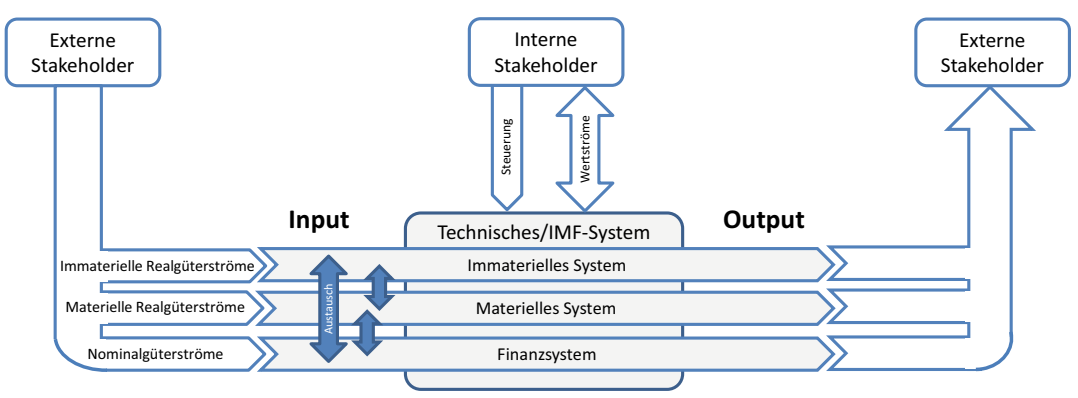

Abbildung 4.9 Das technische System eines Unternehmens als IMF-System ${ }^{464}$

Neben diesen konzeptionellen Grundlagen ergeben sich insbesondere im Kontext der Wissensgesellschaft einige interessante Implikationen, welche im Folgenden noch kurz skizziert seien. So zeigt die klassische Literatur nach wie vor eine vorherrschende Auffassung, technische Systeme lediglich als trivial zu beschreibende Einheiten (s. g. Trivialmaschinen) aufzufassen, welches eine vollständige, externalistische Kontrollmöglichkeit implizieren und im Sinne VON FOERSTERS ein deterministisches, d. h. wohlprognostizierbares Verhalten unterstellen würde. ${ }^{465}$ Der Mensch bzw. das soziale Systeme träte in diesem

463 So führt z. B. ein Kauf von Rechten oder Maschinen zu einem Abfluss an Nominalgütern und einem Zufluss bei den anderen technischen Systemen.

${ }^{464}$ Lingnau, V. / Beham, F. / Fuchs, F. (2020), S. 43.

${ }^{465}$ Vgl. hierzu nochmals Foerster, H. v. (2003), S. 309-313; Foerster, H. v. (1997), S. 37-41; Foerster, H. v. (1984a), S. 201-202; Foerster, H. v. (1984b), S. 8-13. 
Zusammenhang folglich als Instanz auf, welche die technischen Einrichtungen vollständig deterministisch zu kontrollieren vermag. Allerdings muss festgehalten werden, dass im Rahmen der Digitalen Transformation technische Einrichtungen, obgleich immer noch zentral durch allopoietische Eigenschaften gekennzeichnet, in gewissen Grenzen zunehmend Autonomie gewonnen haben bzw. auch weiter gewinnen werden, welches sich darin manifestiert, dass Maschinen bzw. Anlagen und deren Software im Rahmen der s. g. Industrie 4.0 auch partiell eigenständige Optimierungsentscheidungen durchführen können. ${ }^{466}$ Ein bekanntes, in diesem Zusammenhang häufig aufgeführtes Beispiel ist die Smart Factory, in welcher sich Softwareapplikationen und damit verknüpfte materielle Produktionssysteme relativ autonom an dynamische Produktionsanforderungen anpassen können. ${ }^{467}$ Die Fertigungsanlagen sowie die Logistiksysteme verfügen damit über die Möglichkeit, sich in gewissen Grenzen quasi selbst zu organisieren und auf veränderte Rahmenbedingungen eigenständig zu reagieren. Als technische Grundlage dieser Systeme können dabei die cyber-physischen Systeme [CPS] aufgefasst werden, welche wiederum mithilfe des Internets der Dinge und Dienste unterschiedlich stark strukturierte Datenmengen bzw. (mittlerweile) vielfach Wissen ${ }^{468}$ austauschen. Diese CPS können wiederum als vollautomatisierte Produktionseinheiten auftreten, welche mittels ihrer Systemkomponenten, d. h. insbesondere unter Zuhilfenahme von embedded systems ${ }^{469}$, in der Lage sind, mit diversen Komponenten des IMF-Systems (z. B. Maschinen und Anlagen, aber auch Software bzw. Parametereinstellungen) zu interagieren bzw. diese zu beeinflussen. Die nachfolgende Abbildung 4.10 fasst diese Überlegungen sowie die zentralen Entwicklungsstufen zur Industrie 4.0. nochmals zusammen.

\footnotetext{
${ }^{466}$ Aus ethischer Perspektive sei jedoch festgehalten, dass trotz dieser partiellen Autonomie der technischen Systeme immer eine Letztverantwortung für die technisch realisierten Tätigkeiten beim Menschen, bzw. wie aufgezeigt wurde, im Rahmen komplexer technischer Prozesse, bei kollektiven Einheiten liegt. So verbleibt für den Menschen bzw. das Kollektiv immer noch mindestens die Verantwortung, begründete Rahmenbedingungen zu etablieren, welche einen legitimierten Betrieb der technischen Subsysteme gewährleistet sowie z. B. einen menschlichen Letzteingriffsvorbehalt einzurichten.

${ }^{467}$ Vgl. Steven, M. / Dörseln, J. N. (2020), S. 9-12; Lingnau, V. / Brenning, M. (2018), S. 139-140; Burger, A. / Lang, A. / Müller, Y. (2017), S. 58-63; Günthner, W. / Klenk, E. / Tenerowicz-Wirth, P. (2017), S. 97-98; Kreutzer, R. T. (2017), S. 49-51.

468 Zum Wissensbegriff vgl. nochmals die detaillierten Ausführungen in Abschnitt 4.2.4.2.

${ }^{469}$ Diese stellen wiederum kleinste, in physische Objekte integrierte Recheneinheiten bzw. Mikroprozessorik zur Steuerung, Regelung, Überwachung und Datenverarbeitung dar. Vgl. Hüning, F. (2019), S. 11-17; Steven, M. (2019), S. 85; Obermaier, R. (2017), S. 12-13; Lingnau, V. / Brenning, M. (2015), S. 456.
} 


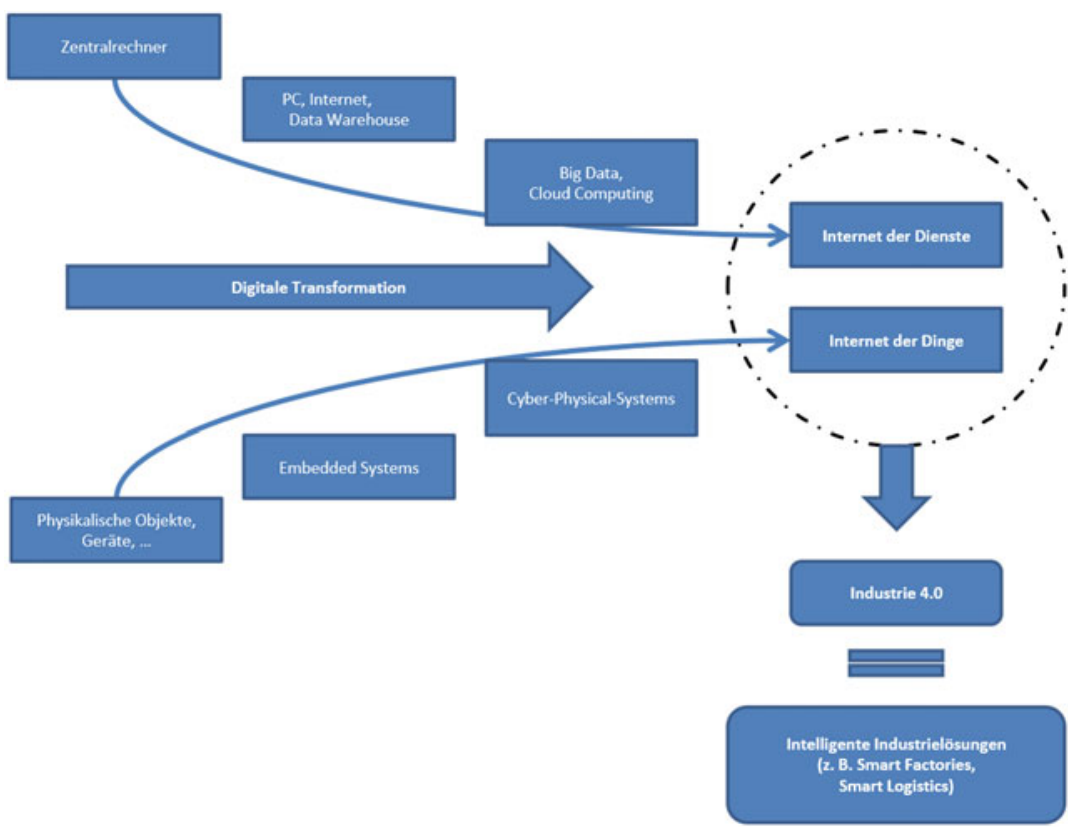

Abbildung 4.10 Entwicklungsstufen der Digitalen Transformation zur Industrie 4.0 $0^{470}$

\subsubsection{Gesamtmodell des Unternehmens}

Die vorausgegangenen Überlegungen können nun genutzt werden, um das Unternehmen als sozio-technisches System, d. h. als Gesamtsystem, welches sowohl das soziale wie technische Teilsystem zu einem Ganzen integriert, zu konzipieren. Dabei ist jedoch festzuhalten, dass noch nicht jedes sozio-technische System sinnvollerweise bereits als „Unternehmen“ bezeichnet werden kann. So sind z. B. kurzlebige Zusammenkünfte, wie Demonstrationszüge oder gesellige Runden, welche unter Einbezug technischer Mittel realisiert werden, evident noch keine Unternehmen, so dass in diesem Zusammenhang offensichtlich noch weitere konstitutive Elemente hinzutreten müssen. Neben dem zentralen, bereits diskutierten, charakteristischen Betriebszweck der monetären Wertschöpfung (im primären oder sekundären Sinne $)^{471}$ sind dies im Wesentlichen drei Faktoren:

${ }^{470}$ Lingnau, V. / Brenning, M. (2018), S. 140; Lingnau, V. / Brenning, M. (2015), S. 456.

471 Vgl. hierzu nochmals die Ausführungen in Abschnitt 2.6.4.2. 
Erstens muss der genannte, charakteristische Instrumental- bzw. Betriebszweck vom sozio-technischen System für Dritte erkennbar über eine gewisse (Mindest-) Dauer verfolgt werden. Darüber hinaus ist zweitens eine Disposition über knappe Ressourcen (d. h. Wirtschaften), welche mit einem gewissen Mindestprofessionalisierungsgrad (z. B. hinsichtlich der Planmäßigkeit oder dem vorhandenen Wissen und Können) vonstattengeht, charakteristisch. Schließlich ist ein Unternehmen drittens durch die Existenz eines Betreibers gekennzeichnet, welcher gegenüber den Stakeholdern als formaler Träger von Rechten und Pflichten auftritt - so z. B. in Kauf- oder Arbeitsverträgen. ${ }^{472}$ Ein solcher Betreiber ist im privatrechtlichen Kontext die Gesellschaft im rechtlichen Sinne, d. h. z. B. eine Aktiengesellschaft, welche das Unternehmen als sozio-technisches System betreibt. In diesem Sinne kann folglich konstatiert werden:

Unternehmen sind von einem Betreiber betriebene sozio-technische Systeme, die für eine gewisse Dauer (im Sinne des ,, going concern “473) für Dritte erkennbar dem instrumentellen Zweck (Betriebszweck) der monetären Wertschöpfung dienen, es sei denn, dass die Erfüllung dieses Betriebszwecks nach Art und Umfang einen gewissen Professionalisierungsgrad (z. B. hinsichtlich Planmäßigkeit oder Wissen und Können) bei Entscheidungen über den Einsatz der knappen Ressourcen (, Wirtschaften“) nicht erfordert. ${ }^{474}$

Von der Gesellschaft (im rechtlichen Sinne) als Träger rechtlicher Verantwortung ist jedoch wiederum der Träger moralischer Verantwortung zu differenzieren, welcher sich gegenüber den Stakeholdern und deren Ansprüchen im moralischen Sinne zu verantworten hat. Letzterer (moralischer) Verantwortungsträger ist (neben weiterhin bestehenden Einzelaspekten der Individualverantwortung), vor dem aufgezeigten Hintergrund einer zunehmenden Komplexität der globalisierten Wirtschaftswelt, vor allem das soziale System des Unternehmens. ${ }^{475}$ Dabei sei für die weitere unternehmensethische Konzeption paradigmatisch davon ausgegangen, dass das soziale System als organisationale Einheit im institutionellen Sinne existiert, d. h. genuin eigene systemische Ziele besitzt und Ansprüche gegenüber seiner Umwelt entwickeln kann, wobei, wie im Weiteren gezeigt wird, aus ethischer Perspektive besonders der intrinsische Zweck des sozialen Systems,

\footnotetext{
${ }^{472}$ Vgl. Lingnau, V. / Beham, F. / Fuchs, F. (2020), S. 53. Die genannten Kriterien gelten dabei (exkl. des Wertschöpfungszwecks) prinzipiell auch für alle anderen Betriebsarten.

${ }^{473}$ Dieser Ausdruck ist angelehnt an die Regelungen des deutschen Handelsgesetzbuches, welches u. a. den Grundsatz des going concern (,Fortführungsprinzip“) betont (vgl. § 252 Abs. 1, S. 2 HGB (2021)).

${ }^{474}$ Vgl. zur allgemeinen Betriebsdefinition auch Lingnau, V. / Beham, F. / Fuchs, F. (2020), S. 55.

475 Vgl. Lingnau, V. / Fuchs, F. (2019), S. 244.
} 
welcher sich im obersten Ziel der Existenzsicherung manifestiert, als Begründungspunkt von Unternehmensethik herangezogen wird. ${ }^{476}$ Diese Überlegungen subsumiert nochmals die nachfolgende Abbildung 4.11.

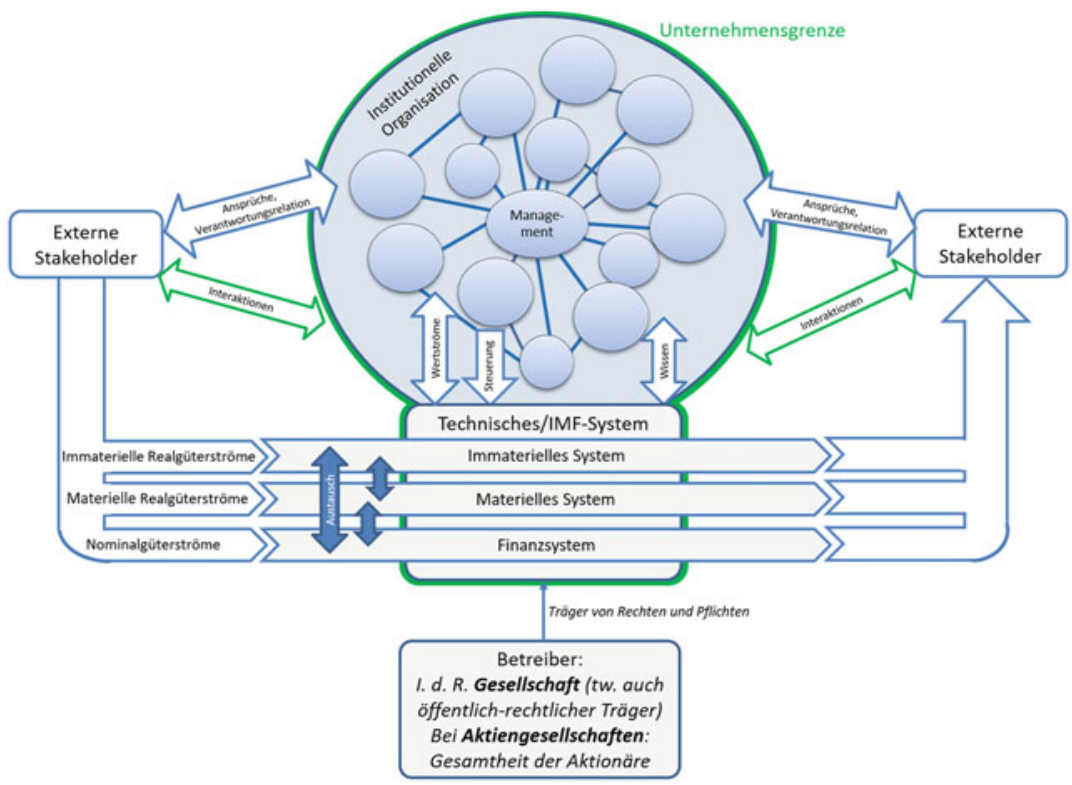

Abbildung 4.11 Das Unternehmen als sozio-technisches System ${ }^{477}$

476 Prinzipiell wäre eine Anwendung der nachfolgenden Überlegungen zu einem strukturierten Legitimitätsmanagement auch für kleine Unternehmen (i. S. kleinerer Mitarbeiterzahl) denkbar. Da dort jedoch möglicherweise (z. B. bei einem Familienunternehmen mit zwei Personen) das soziale System nicht die Komplexität zur Generierung einer eigenen, stabilen systemischen Identität mit Mesozielen erreicht, wäre eine auf ein solches System bezogene Ethik ggf. weniger als genuine Mesoethik, sondern eher als erweiterte Individualethik zu bezeichnen, welche sich stärker auf parallelisierte Individualziele der Individuen bezieht (ähnlich zu den Überlegungen bei HEJL). Zudem befinden sich gerade größere Unternehmen häufiger im kritischen Fokus der Öffentlichkeit und verfügen des Weiteren, ähnlich wie auch bei anderen Managementinstrumenten (z. B. der Balanced Scorecard), eher über die Ressourcen, solche unternehmensethischen Mittel auch regelmäßig einzusetzen.

${ }^{477}$ In Anlehnung an Lingnau, V. / Beham, F. / Fuchs, F. (2020), S. 56. 
Nachdem nun das Unternehmen als spezifisches sozio-technisches Gesamtsystem erarbeitet wurde, stellt sich noch die Frage nach den unternehmerischen Zielen, da ohne diese eine geordnete unternehmerische Tätigkeit bzw. jegliches strukturierte Management ${ }^{478}$ undenkbar wäre. So stellt auch COLSMAN fest: „Steuerung ist nur dort möglich, wo es ein Ziel gibt.“479 Neben dem zentralen Begriff des Ziels sind zudem noch aus Gründen der terminologischen Präzision die verwandten Begrifflichkeiten des Zwecks sowie des Anspruchs zu klären. Dies erfolgt im nachfolgenden Abschnitt.

\subsubsection{Ziele, Zwecke und Ansprüche des Unternehmens}

\subsubsection{Begriffliche Grundlagen}

Betrachtet man zuerst den Begriff des Ziels, so lässt sich konstatieren, dass Ziele in der betriebswirtschaftlichen Literatur vielfach als erwünschte zukünftige Zustände definiert werden. ${ }^{480}$ Fasst man diese Ziele wiederum hierarchisch zusammen, so entsteht ein System miteinander über verschiedene Ebenen verknüpfter Ziele, welche folglich das unternehmerische Zielsystem bilden. Dabei ist zu konstatieren, dass der Begriff der „Unternehmensziele“ eigentlich in einem ersten Schritt vor allem die Ziele des sozialen Teilsystems bzw. der institutionellen Organisation als Kollektiv umfasst ${ }^{481}$, schließt man einmal aus, dass technische Elemente genuin eigene Ziele aufweisen können. Daher sei nun in einem ersten Schritt auf die Entstehung von Zielen im organisationalen Kontext eingegangen. Zur Konzeption von kollektiven Zielen hat die Literatur diverse Ansätze

\footnotetext{
${ }^{478}$ Vgl. hierzu die Ausführungen in Abschnitt 4.4.1.

${ }^{479}$ Colsman, B. (2016), S. 40.

${ }^{480}$ Vgl. Hagenloch, T. (2018), S. 28; Weber, W. / Kabst, R. / Baum, M. (2018), S. 90; Eichhorn, P. / Merk, J. (2016), S. 177; Jung, H. (2016), S. 175; Schweitzer, M. (2009a), S. 9; Töpfer, A. (2007), S. 73; Sturm, R. (2006), S. 47; Stüdemann, K. (1993), S. 231; Kirsch, W. (1974), S. 172 sowie den Überblick bei Macharzina, K. / Wolf, J. (2018), S. 206; Freiling, J. / Reckenfelderbäumer, M. (2010), S. 350.

${ }^{481}$ Eine solche Begriffsverwendung mag auch darauf zurückzuführen sein, dass Unternehmen in der betriebswirtschaftlichen Forschung, so denn eine intensivere konzeptionelle Auseinandersetzung stattfindet, zumeist immer noch stark als soziale Entitäten aufgefasst werden. Vgl. bspw. Weber, W. / Kabst, R. / Baum, M. (2018), S. 5; Jung, H. (2017), S. 3 sowie klassisch Gaugler, E. / Weber, W. (1976), S. 4; Gutenberg, E. (1962), S. 55; Seyffert, R. (1956), Sp. 736.
} 
hervorgebracht, wobei für die weiteren Ausführungen auf eine Taxonomie zurückgegriffen sei, welche ursprünglich auf die Überlegungen von MARSCHAK und RADNER zurückgeht und später nochmals bei RAPOPORT im Überblick systematisiert und präzisiert wurden. ${ }^{482}$ Basierend auf deren Überlegungen kann zwischen vier verschiedenen Ausprägungen sozialer Systeme hinsichtlich der Organisiertheit des Systems und der Frage des Verhältnisses von Kollektiv- und Individualzielen differenziert werden. Den sozialen Zusammenschluss mit der geringsten Kohäsion der Organisiertheit kann in diesem Sinne als Spiel bezeichnet werden. Hierbei verfügen lediglich die interagierenden Individuen über eigene Ziele, welche sie im Rahmen gegebener Spielregeln mehr oder minder bewusst verfolgen. Ein übergeordnetes systemisches Ziel ist demnach nicht vorhanden. Auf der nächsten Stufe befindet sich im Rahmen dieser Taxonomie dann die Koalition - ein Begriff, welcher sich ähnlich auch in der Koalitionstheorie von CYERT und MARCH wiederfindet. ${ }^{483}$ In diesem Fall weisen die Koalitionsteilnehmer sowohl eigenständige Zielsetzungen auf als auch Kollektivziele, welche aus Verhandlungs-, Kontroll- und Anpassungsprozessen der Teilnehmer der Koalition resultieren. Formal können nach LINGNAU und HÄRTEL diese Prozesse vektoranalytisch dargestellt werden, wobei sich die Kollektivziele als Resultante je nach Wirkmächtigkeit der einzelnen Koalitionsteilnehmer ergeben. ${ }^{484}$ Diese Überlegung visualisiert nochmals Abbildung 4.12, welche beispielhaft die Entstehung des Verhandlungsziels ,a“ aus den bewusst repräsentierten Zielen der Organisationsteilnehmer bzw. Suborganisationsgruppen x, y, z, je nach deren Einfluss (hier: $\mathrm{a}_{\mathrm{x}}, \mathrm{a}_{\mathrm{y}}$ und $\mathrm{a}_{\mathrm{z}}$ ), aufzeigt.

482 Vgl. hierzu grundlegend Marschak, J. / Radner, R. (1972), S. 9 und S. 123-126 sowie Rapoport, A. (1986), S. 149-153.

${ }^{483}$ Vgl. Cyert, R. M. / March, J. G. (2001), S. 31-37.

${ }^{484}$ Vgl. Lingnau, V. / Härtel, I. (2014), S. 1-2. 


\section{Abbildung 4.12}

Koalitionsziele als

Resultierende durch

Verhandlungs-, Kontroll-

und Anpassungsprozesse ${ }^{485}$

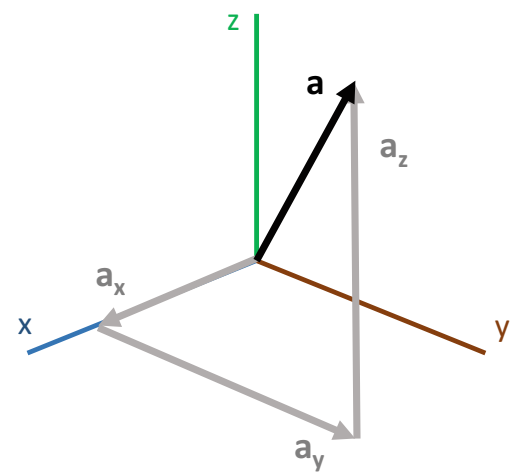

Die nächste Stufe der sozialen Organisiertheit kann dann als Stiftung bezeichnet werden. Hierbei existieren nicht nur individuelle wie auch kollektiv ausgehandelte Ziele, vielmehr kann die soziale Einheit selbst eigenständige (genuine) Systemziele hervorbringen ${ }^{486}$, welche mit den Individual- bzw. Koalitionszielen korrespondieren können, aber nicht müssen. ${ }^{487}$ Schließlich kann als letzte Stufe der sozialen Organisiertheit mit dem Team ein zielhomogenes Kollektiv beschrieben werden, in welchem folglich die organisationalen Ziele mit den Individualzielen vollständig zusammenfallen.

Für die Ausführungen dieser Arbeit erscheint dabei insbesondere das Konzept der Stiftung besonders fruchtbar, da dieses an die Autopoiesisbetrachtung der Organisation als quasi-lebendes System anschlussfähig ist. So weist gerade dieses Konzept am ehesten die Eigenschaften eines Organismus auf, wie auch RAPOPORT feststellt: ,Finally, a foundation may be regarded as a type of organization most resembling an organism. “488 In diesem Sinne weist das soziale System des Unternehmens als institutionelle Organisation sowohl Individual-, als auch gemeinsame Verhandlungsziele auf, allerdings auch eigenständige organisationale

485 Lingnau, V. / Härtel, I. (2014), S. 2.

${ }^{486}$ Die Entstehung genuiner Systemziele kann wiederum mit dem Phänomen der Emergenz erklärt werden. Vgl. hierzu nochmals die Ausführungen in Abschnitt 4.3.3.2.

${ }^{487}$ Ziele können dabei inhaltlich in verschiedenen Beziehungen zueinander stehen. Zu möglichen Zielbeziehungen wie Komplementarität, Indifferenz oder Konkurrenz vgl. Thommen, J.-P. et al. (2020), S. 51; Wöhe, G. / Döring, U. / Brösel, G. (2020), S. 68; Bardmann, M. (2019), S. 390; Weber, W. / Kabst, R. / Baum, M. (2018), S. 97-98; Vahs, D. / Schäfer-Kunz, J. (2015), S. 60-61; Hubig, L. (2009), S. 58; Schiemenz, B. / Schönert, O. (2005), S. 34; Heinen, E. (1992), S. 101-102.

${ }^{488}$ Rapoport, A. (1986), S. 151. 
Ziele, welches sich z. B. durch das Potential zur Autopoiesis emergent im obersten Ziel der Existenzsicherung zeigt. ${ }^{489}$ Die vorausgegangenen Überlegungen subsumiert nochmals die nachfolgende Tabelle 4.3.

Tabelle 4.3 Taxonomie verschiedener existenter Zieltypen nach sozialem Systemtyp 490

\begin{tabular}{|l|l|l|l|}
\hline $\begin{array}{l}\text { Organisations- } \\
\text { form }\end{array}$ & $\begin{array}{l}\text { Bewusste/ } \\
\text { Unbewusste } \\
\text { Individualziele }\end{array}$ & $\begin{array}{l}\text { Verhandlungs- } \\
\text { ziele }\end{array}$ & $\begin{array}{l}\text { (Emergente) } \\
\text { Organisations- } \\
\text { ziele }\end{array}$ \\
\hline Spiel & $\mathrm{X}$ & - & - \\
\hline Koalition & $\mathrm{X}$ & $\mathrm{X}$ & - \\
\hline Stiftung & $\mathrm{X}$ & $\mathrm{X}$ & $\mathrm{X}$ \\
\hline Team & - & - & $\mathrm{X}$ \\
\hline
\end{tabular}

Neben dem Begriff des Ziels existiert mit dem Zweckbegriff ein verwandtes Konzept, welches in der Literatur mit vielfältigem Begriffsinhalt und häufig mehr oder minder synonym gebraucht wird. ${ }^{491}$ Wird dagegen eine Systematisierung vorgenommen, so zeigt sich in diesem Zusammenhang eine beachtliche Begriffsheterogenität. Eine bekannte Differenzierung wurde im betriebswirtschaftlichen Kontext z. B. von EICHHORN und MERK vorgenommen, welche Ziele klassisch als erwünschte Zustände, Zwecke jedoch als ,zielorientierte Verwirklichung des Mitteleinsatzes“ ${ }^{\text {492 }}$, also prozessual, auffassen. ${ }^{493}$ HANS ULRICH wiederum differenziert zwischen Zwecken und Zielen, indem er den Zweck als externalistisch konnotiert auffasst, d. h. funktional gegenüber der Umwelt definiert, während die Ziele internalistisch, d. h. durch das System selbst, gesetzt würden. ${ }^{494}$ Beide Positionen weisen allerdings konzeptionelle Limitationen auf ${ }^{495}$, weshalb im Weiteren der Auffassung gefolgt sei, dass der Zweckbegriff im Gegensatz zum Begriff des Ziels weniger beschreibt was erreicht werden soll, sondern wozu bzw. wofür

${ }^{489}$ Vgl. hierzu auch die Ausführungen des nachfolgenden Abschnitts 4.3.6.2.

${ }^{490}$ Lingnau, V. / Beham, F. / Fuchs, F. (2020), S. 37.

${ }^{491}$ Vgl. z. B. Abels, H. (2019), S. 167; Löwe, M. (2003), S. 56; Conrads, B. (1975), S. 42 sowie im ethischen Zusammenhang auch Wolbert, W. (2011), S. 563.

492 Eichhorn, P. / Merk, J. (2016), S. 177.

493 Vgl. Eichhorn, P. / Merk, J. (2016), S. 176-177.

494 Vgl. Ulrich, H. (1970), S. 114.

495 Vgl. hierzu die Diskussion und die detaillierten konzeptionellen Überlegungen in Lingnau, V. / Beham, F. / Fuchs, F. (2020), S. 28-31. 
etwas getan wird (s. g. „causa finalis“496). Der Zweck kann damit sinnstiftend im Sinne des sozialwissenschaftlichen Begriffs des Verstehens wirken, also gegenüber den Stakeholdern erklären, warum bestimmte Ziele existieren bzw. verfolgt werden. Zwecke sind damit übergreifend bzw. systematisch überdauernd und durchziehen die organisationalen Ziele, indem sie diesen Sinn geben. ${ }^{497}$ Dabei kann weiter zwischen dem Instrumentalzweck und einem systemischintrinsischen Zweck differenziert werden. Der erstere Zweckbegriff beschreibt demnach den instrumentellen Zweck, welcher bei Unternehmen charakteristisch in der ökonomischen Wertschöpfung liegt und zugleich das im Vergleich zu anderen Betriebsformen differenzierende Merkmal $^{498}$ darstellt. Der intrinsische Zweck bezieht sich dagegen auf den Selbstzweck, welcher emergent-autopoietisch bei (quasi-)lebenden Systemen entsteht, sich im Selbsterhalt gegenüber der Umwelt manifestiert und sich im obersten Ziel der Existenzsicherung des Systems gegenüber seiner Umwelt ausdrückt. ${ }^{499}$ So betont auch KLEINFELD: „Die oberste, im Rahmen ihrer jeweiligen systemspezifischen Rationalität gebildete Präferenz einer Organisation besteht folglich darin, ihr ,Überleben', d. h. ihren Fortbestand als solche zu sichern." ${ }^{\text {"500 }}$ Auch SIMON stellt in diesem Zusammenhang fest: „Überleben wird dabei zum Rationalitätskriterium des Handelns.“501

${ }^{496}$ Der Begriff der causa finalis als Zweckursache stammt von ARISTOTELES, welcher ihn im Zusammenhang mit den anderen drei Ursachen der causa materialis (Stoffursache), causa formalis (Formursache) sowie der causa efficiens (Wirkursache/Kausalität) diskutierte. Vgl. Aristoteles (1989), 1013a; Aristoteles (1987), 194a-195b.

497 Ähnliche Überlegungen finden sich auch bei DoLL, welcher feststellt: „Denn im Zweck bestimmt sich, warum das System überhaupt existiert.“ (Doll, A. (2016), S. 29). Darüber hinaus und in Abgrenzung von Zwecken und Zielen konstatiert dieser: „Im Gegensatz zum Unternehmenszweck, der eine Antwort auf die Sinn-Frage eines Faktums ist (z. B. der Zweck eines Hammers ist es, Nägel einzuschlagen [...]), ist ein Ziel der Ausdruck eines Wollens oder einer Hoffnung auf einen zukünftigen Zustand hin.“ (Doll, A. (2016), S. 30).

498 So können Unternehmen durchaus auch noch weiteren Zwecken wie z. B. der persönlichen Entfaltung, der Sinnstiftung oder der Bildung dienen. Der Wertschöpfungsfokus stellt jedoch das im Vergleich zu anderen Betriebstypen differenzierende Merkmal in der instrumentellen Zwecksetzung dar.

499 Je nach eingenommener Perspektive stehen beide Zwecke in einer Zweck-MittelHierarchie. So ist aus Sicht der Stakeholder zur kontinuierlichen Realisierung des Betriebszwecks das Überleben des Unternehmens (und damit auch dessen sozialen Teilsystems) notwendig. Umgekehrt ist aus Perspektive des sozialen Systems die legitime Erfüllung des Betriebszwecks notwendig, um die eigene Existenz auf Dauer zu sichern und dient damit dem intrinsischen Zweck.

500 Kleinfeld, A. (1998), S. 332-333.

${ }^{501}$ Simon, F. B. (2019), S. 32. 
Schließlich resümiert auch WOLF: „Oft wird in der Systemerhaltung bzw. dem Systemüberleben das wichtigste Ziel von Organisationen gesehen. "502

Nach der erfolgten Abgrenzung von Zwecken und Zielen ist nun ebenfalls der Begriff des Anspruchs zu klären, welcher im betriebswirtschaftlichen Schrifttum meist relativ unsystematisiert gebraucht wird. Dabei sei im Folgenden auf ein teleologisches Anspruchsverständnis rekurriert, welches grundlegende Vorüberlegungen in der rechtlichen Sphäre findet. So definiert $§ 194$ Abs. 1 BGB einen Anspruch als ,[d]as Recht, von einem anderen ein Tun oder Unterlassen zu verlangen [...]."503 Ein solches Anspruchskonzept umfasst allerdings nur rechtlich kodifizierte Ansprüche, so dass unkodifizierte, moralisch begründete Ansprüche vernachlässigt würden. Zudem fehlen in der rein legalistischen Definition gegen sich selbst gerichtete Ansprüche (Selbstansprüche), welche ebenfalls in eine Anspruchskonzeption aufgenommen werden sollten. Die vorausgegangenen Überlegungen führen damit unter Rückgriff auf den Zielbegriff zu folgendem, teleologischen Anspruchsverständnis: Ansprüche ergeben sich aus den Zielen eines Anspruchsträgers. Sie sind gegen einen Dritten oder sich selbst gerichtet und beziehen sich auf das Tun oder Unterlassen einer Entscheidung oder Handlung. ${ }^{504}$

Ansprüche resultieren folglich aus einer Transformation von Zielen mittels Adressatenbezug, wobei Selbstansprüche durch tautologische und Fremdansprüche durch einfache Transformation aus Zielen entstehen. Kompakt könnten Ansprüche somit auch als gerichtete Erwartungen einer Zielerfüllung bezeichnet werden. Dabei existieren jedoch nicht nur monokausale Verbindungen von Zielen zu Ansprüchen. Vielmehr können realiter auch zwischen den Ansprüchen wiederum selbst Beziehungen entstehen. Ein kurzes Beispiel möge dies verdeutlichen: So kann beispielsweise aus dem Ziel, das Studium mindestens mit „gut“ abzuschließen, ein Selbstanspruch, dieses Ziel auch zu erreichen, entwickelt wer$\operatorname{den}^{505}$, wobei auch weitere, derivative Selbstansprüche, z. B. fleißig zu lernen, entstehen können. Aus dem Selbstanspruch können zudem Ansprüche an Dritte entwickelt werden, welche z. B. an die Lehrkräfte, Kommilitonen, Eltern etc. gerichtet werden und darauf abzielen, das genannte Ziel verwirklichen zu helfen (d. h. ,etwas zu tun“) oder zumindest dieses nicht zu stören (d. h. ,etwas zu

\footnotetext{
502 Wolf, J. (2013), S. 192.

$503 \S 194$ Abs. 1 BGB (2021).

${ }^{504}$ Vgl. ähnlich Lingnau, V. / Beham, F. / Fuchs, F. (2020), S. 31.

505 Vergleichbare Überlegungen existieren im Rahmen der Motivationstheorie auch konzeptionell zwischen Motiven als Disposition, während erst ,aktivierte“ Motive zur Motivation führen. Vgl. z. B. grundlegend Rheinberg, F. / Vollmeyer, R. (2019), S. 77-78; Heckhausen, J. / Heckhausen, H. (2018), S. 4-7; Rosenstiel, L. v. (2015), S. 6; Rosenstiel, L. v. / Nerdinger, F. W. (2011), S. 238; Schmalt, H.-D. / Langens, T. A. (2009), S. 18-38.
} 
unterlassen“). Die aufgezeigte Systematisierung fasst nochmals die nachfolgende Abbildung 4.13 zusammen.

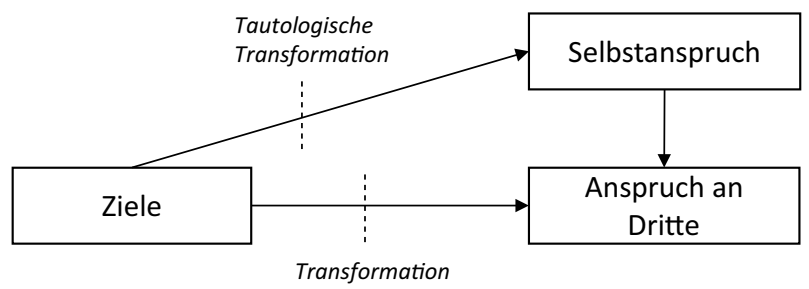

Abbildung 4.13 Entstehung von Ansprüchen durch (tautologische) Transformation von Zielen $^{506}$

\subsubsection{Implikationen für das organisationale Zielsystem und die Bedeutung normativer Ziele}

Die vorausgegangenen Überlegungen können nun auf das organisationale Zielsystem übertragen werden, wobei davon ausgegangen wird, dass sich wiederum innerhalb des Zielsystems eine hierarchische Ordnung der Ziele anhand des für die jeweilige Ebene charakteristischen Oberziels erstellt werden kann. Diese übergeordneten Ziele können wiederum innerhalb der gleichen Hierarchiestufe weitere untergeordnete Zielsetzungen enthalten, welche im Folgenden als Zwischenziele und Unterziele bezeichnet werden. ${ }^{507}$ Betrachtet man die aktuelle betriebswirtschaftliche Literatur, so zeigt sich nach wie vor häufig eine Differenzierung zwischen strategischen und operativen Zielen, wobei ggf. zwischen diesen Zielen noch eine taktische Ebene verortet wird. ${ }^{508}$ Zur Unterscheidung

${ }^{506}$ Lingnau, V. / Beham, F. / Fuchs, F. (2020), S. 32. Dabei können Ansprüche und deren Verwirklichung anhand empirischer Restriktionen freilich im Zeitablauf auch die vorige Zielhöhe, aber auch das Anspruchsniveau verändern. Auf diese Rückkopplungsprozesse sei jedoch aus Vereinfachungsgründen in der Abbildung verzichtet. Zur Adaption der Anspruchs- bzw. Zielhöhe vgl. auch die Ausführungen zur ,aspiration adaptation theory“ in Selten, R. (2001), S. 18-24 sowie grundlegend Selten, R. (1998); Sauermann, H. / Selten, R. (1962).

507 Vgl. Thommen, J.-P. et al. (2020), S. 51-52; Wöhe, G. / Döring, U. / Brösel, G. (2020), S. 68-69; Bardmann, M. (2019), S. 391; Kußmaul, H. (2016), S. 21; Vahs, D. / Schäfer-Kunz, J. (2015), S. 61; Schiemenz, B. / Schönert, O. (2005), S. 36; Eberhardt, S. (1998), S. 54; Heinen, E. (1992), S. 104-105.

508 Vgl. z. B. Wöhe, G. / Döring, U. / Brösel, G. (2020), S. 73; Hagenloch, T. (2018), S. 370; Macharzina, K. / Wolf, J. (2018), S. 442; Hering, T. (2017), S. 9-11; Kußmaul, H. (2016), 
dieser Ebenen wird für die weiteren Ausführungen der Arbeit folgende Klassifikation zugrunde gelegt: Auf der strategischen Zielebene können alle Ziele, welche den Aufbau sowie den Erhalt von Erfolgspotentialen (bei Unternehmen: Wertschöpfungspotentiale) umfassen, subsumiert werden. Hierin eingebettet bzw. untergeordnet finden sich die operativen Ziele bzw. die Nutzung dieser Erfolgspotentiale. Charakteristisch für die operative Zielebene ist dabei die effektive wie effiziente Nutzung von Erfolgspotentialen zur Generierung von Erfolg, d. h. bei Unternehmen: der regelmäßigen Erzielung einer ökonomischen Wertschöpfung. Obschon diese klassische Zweiteilung nach wie vor eine große Verbreitung in der betriebswirtschaftlichen Literatur aufweist, ist der klassische strategischoperative Ansatz doch als zu restriktiv zu kritisieren. So vernachlässigt dieser die normativen Grundlagen, welche unternehmerischem Handeln immer schon zugrunde liegen, denn dieses Handeln findet klarerweise immer bereits in durch Werte präformierten Erwartungshaltungen der Gesellschaft als organisationaler Umwelt statt. Gerade vor dem Hintergrund zunehmend kritischer Stakeholder ist zu konstatieren, dass eine rein strategisch-operative Ausrichtung Gefahr läuft, nicht zu prüfen, inwiefern Strategien sowie operative Entscheidungen bzw. Handlungen überhaupt normativ zulässig sind, denn, wie gezeigt wurde, ist in einer freiheitlichen Gesellschaft noch nicht jedes strategische wie auch operative Handeln bereits selbstverständlich hinreichend legitimiert. Eine Missachtung der normativen Sphäre unternehmerischer Entscheidungen, kann dabei, wie etliche Unternehmensskandale aufzeigen, mit gravierenden und sogar existenziellen Konsequenzen einhergehen. ${ }^{509}$

Auf diesen Überlegungen aufbauend ist damit die klassische Zweiteilung des organisationalen Zielsystems zu erweitern, so dass in einer übergeordneten Ebene auch die normativen Voraussetzungen des wirtschaftlichen Handelns

S. 152; Vahs, D. / Schäfer-Kunz, J. (2015), S. 341; Jung, H. (2014), S. 436; Peters, S. / Brühl, R. / Stelling, J. N. (2005), S. 163; Palupski, R. (2002), S. 83. Dabei wird jedoch gerade die taktisch-operative Ebene häufig auch wieder zusammengefasst (vgl. z. B. Balderjahn, I. / Specht, G. (2020), S. 195; Berndt, R. / Altobelli, C. F. / Sander, M. (2016), S. 20; Irle, C. (2011), S. 48; Steinle, C. (2005), S. 292), so dass im Rahmen der Arbeit auf eine weitere Differenzierung verzichtet sei. Vgl. hierzu auch Hansmann, K.-W. (2006), S. 25.

${ }^{509}$ Vgl. zum Überblick Lingnau, V. / Kokot, K. (2015), S. 29-44. 
beleuchtet und konzeptionell abgebildet werden. ${ }^{510}$ Diese Ebene wird in Anlehnung an die Überlegungen der St. Galler Schule ${ }^{511}$ als normative Zielebene bezeichnet. Als normatives Oberziel dieser Ebene wird dabei der Aufbau und der Erhalt von Legitimität konzipiert, welcher, wie im Weiteren gezeigt wird, zum Ausgangspunkt des normativen Managements ${ }^{512}$ als strukturiertem Legitimitätsmanagement und zur Begründung von Unternehmensethik als intrinsisch relevanter Verantwortungsübernahme genommen werden kann. Alle drei Ebenen, d. h. die normativen, strategischen wie operativen Ziele, werden schließlich von einem obersten Ziel geprägt, welches auch als systematisches Metaziel oder Basalziel bezeichnet werden könnte, das die fundamentale Grundlage allen unternehmerischen Handelns bildet und besonders durch den aufgezeigten intrinsischen Zweck des sozialen Systems begründet wird. Dieses ist das Ziel der Existenzsicherung, welches letztlich allen normativen, strategischen als auch operativen Zielsetzungen inhärent ist. In diesem Sinne stellt auch HERING fest: „Als oberstes Ziel eines Unternehmens kann die langfristige Sicherung seines Fortbestands angesehen werden." 513 Ebenso hebt auch DiCKMANN hervor: „Das oberste Unternehmensziel stellt nach herrschender Literaturmeinung die langfristige Existenzsicherung des Unternehmens dar." ${ }^{\text {514 }}$ Ähnlich betonen schließlich auch ZDROWOMYSLAW und KASCH ,,[d]ie Unternehmenssicherung (Überleben)

\footnotetext{
510 Teilweise wird in der Literatur an dieser Stelle noch eine Langfristplanung als den strategischen Zielen übergeordnete Ebene postuliert, welche dann z. B. auf Visionen oder Leitideen aufbauen (vgl. z. B. Reber, M. / Pifko, C. (2018), S. 105; Weber, W. / Kabst, R. / Baum, M. (2018), S. 92). Eine solche Konzeption hätte jedoch den Nachteil, dass, neben einer primär zeitlichen und nicht inhaltlichen Ausrichtung, die Visionen oder Leitideen selbst nicht normativ reflektiert, $d$. h. als gegeben angenommen werden, welches wiederum der hier vorgeschlagenen normativen Zielebene zur kritischen Reflexion bedürfte.

${ }^{511}$ Vgl. hierzu auch die detaillierteren Überlegungen in Abschnitt 4.4.1.

512 Zur Vollständigkeit sei erwähnt, dass auch rechtliche Überlegungen eine gewisse Rolle auf normativer Ebene einnehmen. Da sich die Arbeit jedoch im Kern mit unternehmensethischen (und nicht -rechtlichen) Fragestellungen befasst und die Einhaltung von Rechtsnormen nur bedingt eine ethische Fragestellung repräsentiert, seien rechtliche Evaluationen für das normative Management im Rahmen der weiteren Ausführungen weitestgehend ausgeblendet.

${ }^{513}$ Hering, T. (2017), S. 9. Es sei nochmals angemerkt, dass im ersten Schritt dies das Ziel des sozialen Teilsystems des Unternehmens darstellt. Zum genaueren Zusammenhang zwischen dem sozialen Zielsystem und dem gesamtunternehmerischen Zielsystem vgl. auch den folgenden Abschnitt 4.3.6.3.

${ }^{514}$ Dickmann, T. (2010), S. 102. Vgl. ähnlich auch Wittenberg, V. (2006), S. 45-46.
} 
als oberstes Ziel [...]." ${ }^{515}$ Dabei dienen letztlich alle drei Zielebenen (Legitimität, Wertschöpfungspotentiale, Wertschöpfung) diesem obersten Ziel. So ist die langfristige Existenzsicherung nur möglich, wo das Unternehmen legitimierte Wertschöpfungspotentiale entwickelt und diese in einem legitimen Sinne effektiv wie effizient nutzt, so dass stets alle der drei aufgezeigten Zielebenen Berücksichtigung finden müssen. Es sei daher nochmals betont, dass sowohl strategische als auch operative Entscheidungen normativ überzeugend fundiert sein müssen. Diese Überlegungen fasst nochmals die nachfolgende Abbildung 4.14 zusammen.

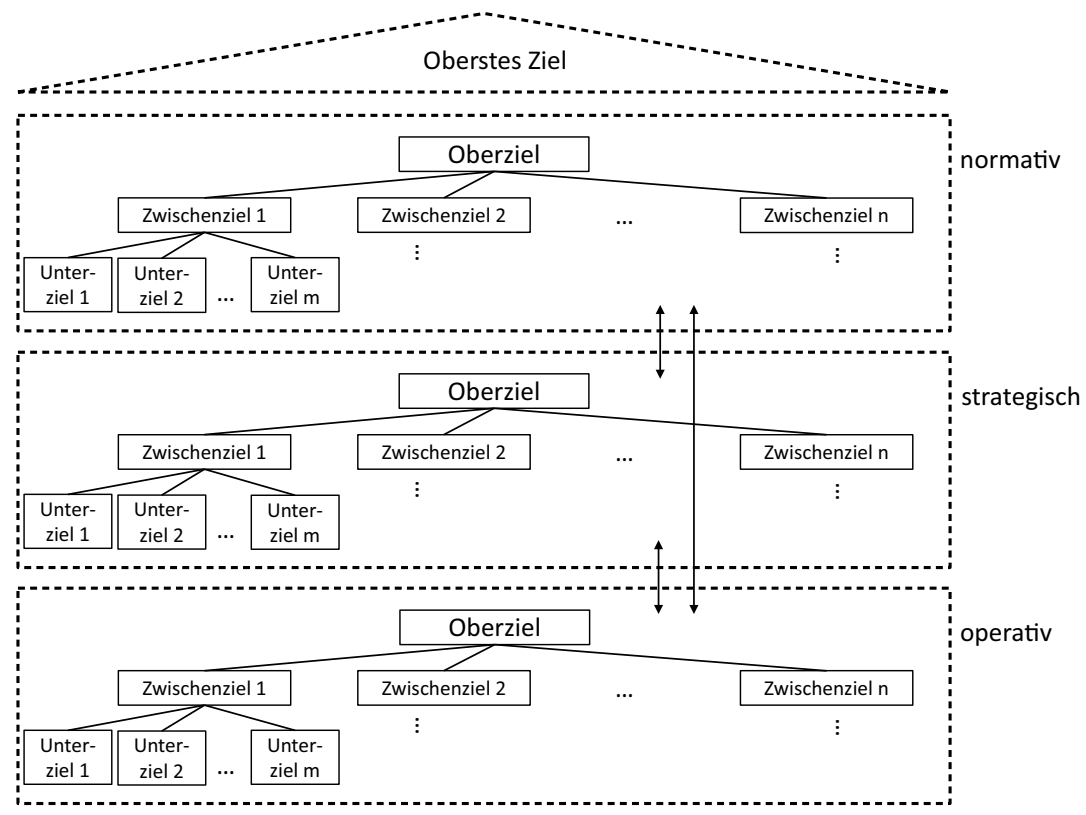

Abbildung 4.14 Die organisationalen Zielebenen im Überblick

515 Zdrowomyslaw, N. / Kasch, R. (2002), S. 8, in der Quelle fett herv. Pointiert stellt auch COLSMAN fest: „Neben vielen anderen Zielen ist das oberste Ziel eines Unternehmen die langfristige Existenzsicherung, um dauerhaft seinen Zweck [...] erfüllen zu können." (Colsman, B. (2016), S. 40). Vgl. auch Ebner, R. (2015), S. 113; Burggraf, A. (2012), S. 20; Baier, P. (2008), S. 384; Lienhard, P. (2008), S. 190; Christians, V. C. (1985), S. 32-33; Grimm, U. (1983), S. 250. 
Aus den Zielen der genannten Ebenen lassen sich wiederum, wie gezeigt wurde, durch Transformation bestimmte organisationale Ansprüche ableiten. Ansprüche können dabei, basierend auf den Überlegungen von DAHRENDORF ${ }^{516}$, sowie hierauf aufbauend WILLENBACHER ${ }^{517}$, je nach Konkretisiertheit von Inhalt, Ausmaß sowie zeitlichem Bezug ${ }^{518}$ weiter in drei Arten differenziert werden. Während Interessen generell ein eher niedriges Niveau an Konkretisiertheit aufweisen, sind Forderungen bezüglich ihres Inhalts, Ausmaßes sowie zeitlichen Bezugs relativ stark konkretisiert. Ansprüche, welche hinsichtlich der genannten Spezifikation ein mittleres Niveau aufweisen, werden schließlich als Anliegen bezeichnet. Dies verdeutlicht nochmals die nachfolgende Abbildung 4.15.

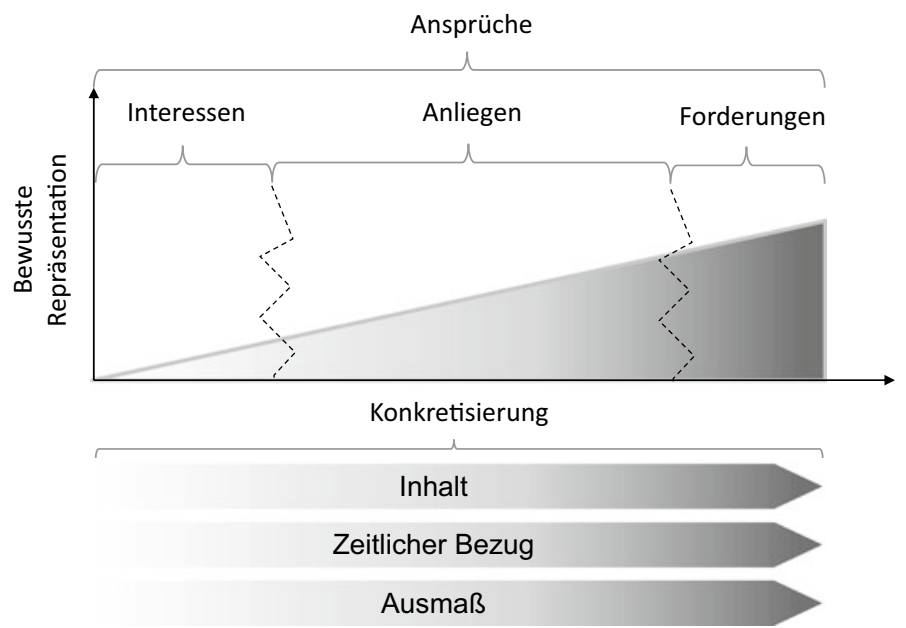

Abbildung 4.15 Überblick über die drei Anspruchstypen ${ }^{519}$

Basierend auf diesen Überlegungen können nun nochmals die Beziehungen zwischen Zielen und Ansprüchen genauer untersucht werden. Ziele der obersten Ebene, wie sie sich z. B. im Überleben oder im Aufbau wie Erhalt der Legitimität

${ }^{516}$ Vgl. Dahrendorf, R. (1972b), S. 47-63.

517 Vgl. Willenbacher, P. (2017), S. 23.

518 Diese Klassifikation geht auf HEINEN zurück, welcher sie für operationale Zielsetzungen einführte. Vgl. hierzu Heinen, E. (1992), S. 98-101; Heinen, E. (1976), S. 59-89.

${ }^{519}$ Lingnau, V. / Beham, F. / Fuchs, F. (2020), S. 33. Vgl. grundlegend auch Willenbacher, P. (2017), S. 23. 
wiederfinden, sind meist eher abstrakt bzw. geringer spezifiziert und dürften dabei häufig zu Interessen, wie bspw. dem Überlebensinteresse oder dem Interesse des Erhalts der gesellschaftlichen Legitimität führen. Ziele mittlerer Konkretisiertheit, wie bspw. im strategischen Bereich, korrespondieren dann folglich eher mit dem Anspruchstyp des Anliegens, während aus operativen Zielen häufig konkrete Forderungen entstehen dürften. Abschließend sei allerdings festgehalten, dass eine solche Zuweisung eher idealtypisch aufzufassen ist, denn je nach genauer Situation können bspw. auch aus den höheren organisationalen Zielebenen sehr konkrete Ansprüche resultieren. So ist es z. B. denkbar, dass sich aus dem Ziel der Existenzsicherung bzw. des Erhalts von Legitimität in Krisenzeiten auch sehr konkrete Ansprüche, d. h. Forderungen, gegenüber den Stakeholdern entwickeln, bspw. illegitimes, schädigendes Verhalten zu unterlassen. 520

\subsubsection{Schlussfolgerungen für das sozio-technische Gesamtsystem}

Im Folgenden können die bisher primär organisational fokussierten Ausführungen auch nochmals konkret in Beziehung zum sozio-technischen Gesamtsystem gesetzt und konzeptionell vertieft werden. Hierbei sind insbesondere noch die Rolle des technischen Systems sowie die Implikationen auf die Eigenschaften des Gesamtsystems zu klären. Hinsichtlich des Zielbegriffs kann zum einen festgestellt werden, dass technische Systeme von Menschen (bzw. anderen kunstfertigen Wesen $^{521}$ ) zur Realisierung von individuellen wie kollektiven Zielen geschaffen werden. Technische Systeme besitzen damit in einem ersten Schritt keine originär eigenständigen Ziele, sondern sind auch im unternehmerischen Kontext den organisationalen Zielen dienend gestaltet. Wie die Ausführungen im Rahmen der Industrie 4.0 zeigten, erscheint es jedoch klar, dass in einem derivativen Sinne aus den vordefinierten bzw. vorgeprägten organisationalen Zielen das technische System partiell auch eigenständig Subziele bilden kann. So ist z. B. plausibel, dass ein durch die Hard- und Software relativ selbständig optimierter Routenplan durchaus technisch relativ autonom definierte Ziele enthält, welche bezüglich ihrer aktuellen Realisierung auch von technischen Systemen selbst überwacht werden. Die Letztverantwortung muss jedoch stets beim sozialen System als

\footnotetext{
${ }^{520}$ So weist im unternehmerischen Kontext auch FASSIN auf die Bidirektionalität von Ansprüchen hin. In diesem Sinne haben nicht nur Stakeholder (legitime) Ansprüche gegenüber dem Unternehmen, sondern auch das Unternehmen (bzw. genauer dessen soziales Subsystem) hat (legitime) Ansprüche gegenüber seinen Stakeholdern. Vgl. hierzu Fassin, Y. (2012), S. 90-91.

${ }^{521}$ Vgl. hierzu nochmals Abschnitt 4.3.2.
} 
i. d. R. institutioneller Organisation verbleiben, welche auch einen Letztvorbehalt gegenüber den technischen Systemen besitzen muss, wenngleich bei hoher Komplexität der genaue Zielzustand der technischen Systeme im Detail nicht immer transparent sein mag. Das Unternehmen als sozio-technische Gesamtheit enthält damit die Eigenschaften sowohl des sozialen als auch des technischen Systems, wobei bemerkt sei, dass das aktive gestalterische Potential des sozialen Systems im Zielbildungsprozess dominieren und damit das Gesamtzielsystem prägen dürfte. ${ }^{522}$ Ähnlich kann auch im Kontext des Zweckbegriffs argumentiert werden. Technische Systeme dienen hinsichtlich ihres Zwecks der Organisation: zum einen in der Realisierung ihres Instrumentalzwecks (z. B. Wertschöpfung), zum anderen aber auch im Sinne des intrinsischen Zwecks zur organisationalen Selbsterhaltung. Bei letzterem kann zudem ebenfalls argumentiert werden, dass für einen außenstehenden Beobachter das Unternehmen vielfach als Einheit auftritt, so dass hierbei z. B. das Interesse zu Überleben von der Organisation auf das Gesamtunternehmen übertragbar wird, insbesondere wenn man bedenkt, dass das soziale Teilsystem in der Regel ohne technische Einrichtungen seine Existenz nicht erhalten kann. Im Rahmen der Ansprüche kann ebenfalls auf diese Überlegungen zurückgegriffen werden. Originär besitzt im Unternehmen sicherlich erst einmal nur das soziale Subsystem genuin Ansprüche gegenüber seiner Umwelt. Technische Systeme werden jedoch zur Übertragung von Ansprüchen sowie zur Sammlung bzw. Analyse von Stakeholderansprüchen organisational genutzt, wobei auch hier wiederum das Unternehmen regelmäßig als artikulierende Einheit von Ansprüchen wahrgenommen wird.

Im Nexus von Ansprüchen, Zielen und hieran orientierten Handlungen nimmt nun insbesondere das Management eine überragende Bedeutung ein, denn dieses ist damit beauftragt, die organisatorischen Rahmenbedingungen so zu setzen, dass das Unternehmen (als Ganzes, d. h. auch hinsichtlich seiner technischen Mittel) seinem Wertschöpfungszweck dauerhaft erfolgreich mit legitimen Mitteln nachkommt. Hierbei ist das Management folglich als zentrale Steuerungsinstanz gefordert, entsprechend perturbierend auf untergeordnete organisationale Ebenen (sowie ggf. auch direkt oder indirekt auf das technische System) so einzuwir$\mathrm{ken}^{523}$, dass die kollektiven und systemischen Ziele dauerhaft legitimiert erreicht werden und das Gesamtsystem „Unternehmen“ stabilisiert wird bzw. erhalten

522 Dies ist auch aus ethischer Perspektive höchst relevant, da technische Systeme keine Verantwortung übernehmen können. Vgl. hierzu auch Lingnau, V. / Fuchs, F. (2019), S. 241. ${ }^{523} \mathrm{Zu}$ den Steuerungsgrenzen komplexer Systeme im Sinne einer Kybernetik erster Ordnung vgl. die detaillierten Überlegungen in Lingnau, V. / Beham, F. / Fuchs, F. (2022). 
bleibt. So stellen auch Hoitsch und LingnaU fest: „Aufgabe des Managements ist es, durch Entscheidungen die leistungs- und finanzwirtschaftlichen Prozesse so zu steuern, dass die betrieblichen Ziele möglichst optimal erreicht werden [...].“524 Ähnlich resümiert auch VON KÄNEL: „Die Realisierung des Geschäftsbetriebes eines Unternehmens [...] bedarf zwingend einer Führung, die ihrerseits Ziele setzt, das arbeitsteilige Handeln der Bereiche des Unternehmens organisiert, plant, koordiniert und damit steuert und die zugleich die erreichten Ergebnisse kontrolliert und analysiert. Diese Aufgabe ist durch das Management des Unternehmens - im Sinne einer zweckbestimmten, zielgerichteten Unternehmensführung - wahrzunehmen [...].“525

Dabei gewinnt, wie im Weiteren ausgeführt wird, neben klassisch strategischoperativen Überlegungen korrespondierend zur Bedeutung der Berücksichtigung der Legitimität unternehmerischen Handelns im Zielsystem des Unternehmens auch die normative Dimension zunehmende betriebswirtschaftliche Relevanz für das Management und kann damit zum Ausgangspunkt der Konzeption einer genuinen Mesoethik genommen werden. Der Begriff des Managements und insbesondere des normativen Managements ist Gegenstand der nachfolgenden Ausführungen, in welchen neben konzeptionellen Grundlagen des normativen Managements auch ein für die Praxis relevantes Instrument zur Integration und Reflexion der Stakeholderansprüche in Bezug auf das unternehmerische Zielsystem entwickelt wird.

\subsection{Normatives Management als systematisches Legitimitätsmanagement}

\subsubsection{Grundlagen des Managementbegriffs}

Der Begriff des Managements weist, obschon seiner neuzeitlichen Anmutung, lange in der Menschheitsgeschichte zurück. So stellt auch COMBE fest: „Management has a long history and dates back to well before it became a subject of formal study. [...] Empires were built on effective management of resources [...] to protect those civilisations. " ${ }^{26}$ Ähnlich subsumieren MOORE und PAREEK:

\footnotetext{
${ }^{524}$ Hoitsch, H.-J. / Lingnau, V. (2007), S. 31, in der Quelle fett herv.

525 Känel, S. v. (2018), S. 314.

${ }^{526}$ Combe, C. (2014), S. 5.
} 
„The idea of management dates back to the beginning of civilization.“527 Eine etymologische Auseinandersetzung mit dem Managementbegriff zeigt dabei, dass dieser vermutlich von den beiden lateinischen Termini manus und agere (,an der Hand führen") herrührt. Die genaue Begriffsherkunft ist jedoch wissenschaftshistorisch bis heute nicht unumstritten, so dass in der Literatur auch andere Wurzeln, z. B. in mansionem agere (,das Haus führen“), postuliert werden. ${ }^{528}$ Das Wort Management, welches im fachwissenschaftlichen Diskurs im angelsächsischen Sprachraum popularisiert wurde ${ }^{529}$, fand dabei ab Mitte des letzten Jahrhunderts zunehmende Verbreitung in der deutschsprachigen betriebswirtschaftlichen Forschung. ${ }^{530}$ In der heutigen Betriebswirtschaftslehre hat sich dabei, analog zu den Ausführungen im Rahmen des Organisationsbegriffs, mittlerweile eine Differenzierung zwischen einer funktionalen und institutionellen Begriffsauffassung weitgehend etabliert. Hierbei beschreibt der funktionale Managementbegriff die Tätigkeit der Unternehmensführung bzw. -steuerung ${ }^{531}$, während der institutionelle Begriff auf die soziale Entität bzw. das organisationale Subsystem rekurriert, welche diese Funktion dominant institutionalisiert bzw. professionalisiert ausübt. ${ }^{532}$

${ }^{527}$ Moore, K. / Pareek, N. (2009), S. 5. Vgl. zur historischen Entwicklung auch die Ausführungen bei Witzel, M. (2017), S. 13-39.

${ }^{528}$ Vgl. Wolf, G. (2010), S. 43; Moore, K. / Pareek, N. (2009), S. 6; Scholl, C. (2008), S. 131; Doede, K. / Bossink, B. A. G. (2007), S. 23; Staehle, W. H. / Conrad, P. / Sydow, J. (1999), S. 71.

${ }^{529}$ Nach MOORE und PAREEK entstand der englische Terminus gegen Ende des 16. Jahrhunderts. Vgl. hierzu Moore, K. / Pareek, N. (2009), S. 6.

${ }^{530}$ Vgl. Scholl, C. (2008), S. 131; Staehle, W. H. / Conrad, P. / Sydow, J. (1999), S. 71.

531 Als Abgrenzungsmerkmal zu anderen allgemeinen unternehmensbezogenen Führungsbzw. Steuerungstätigkeiten (bzw. einem Begriff des „Selbstmanagement“) kann für das Management im Begriffssinne dieser Arbeit die existierende Personalverantwortung herausgestellt werden. Ähnlich stellen so auch DOEDE und BosSINK fest: ,The word ,management“ refers to all the employees in an organization whose job it is to set in motion, prepare and control the actions of other people and resources, given the objectives - implicit or explicit - of the organization.“ (Doede, K. / Bossink, B. A. G. (2007), S. 23).

532 Vgl. Hagenloch, T. (2018), S. 67; Känel, S. v. (2018), S. 315-316; Weber, W. / Kabst, R. / Baum, M. (2018), S. 8; Zischg, K. (2018), S. 69; Schierenbeck, H. / Wöhle, C. B. (2016), S. 113; Heuermann, R. / Tomenendal, M. (2011), S. 22; Hummel, T. R. (2010), S. 99-100; Löffelholz, J. (1980), S. 183. 
Hinsichtlich der Funktion des Managements hat die Literatur eine Vielzahl von Systematisierungen erarbeitet. ${ }^{533}$ Diese reichen von einer verbreiteten Aufgabendifferenzierung nach dem klassischen „Fünferkanon“ des prévoir, organiser, commander, coordonner, contrôler bei $\mathrm{FAYOL}^{534}$, planning, organizing, staffing, leading und controlling bei KOONTZ, O'DONNELL und WEIHRICH ${ }^{535}$ sowie das POSDCORB-Konzept von GULICK, welches die vorigen Systematiken um die Funktion des Reportings sowie der Budgetierung erweitert. ${ }^{536}$ Daneben findet sich in der Literatur auch vielfach eine hierarchische Differenzierung der Managementfunktionen, wobei sich die Managementfunktionsebene jeweils auf die organisationale bzw. unternehmerische Zielebene bezieht, ist das Management doch wie gezeigt mit der Ausbalancierung und Erreichung von unternehmerischen Zielen befasst. ${ }^{537}$ Die klassische Betriebswirtschaftslehre differenziert nun analog zu den Ausführungen zum Zielbegriff wiederum zwischen einem übergeordneten strategischen und untergeordneten operativen Management. ${ }^{538}$ Anknüpfend an die vorigen Überlegungen kann dabei festgestellt werden, dass das strategische Management mit der Zielerreichung auf strategischer Ebene und damit im Kern mit der Schaffung unternehmerischer Wertschöpfungspotentiale befasst ist. Das operative Management wiederum knüpft hieran an und befasst sich mit der Realisierung operativer Zielsetzungen, d. h. der effizienten wie effektiven Nutzung von Erfolgs- bzw. Wertschöpfungspotentialen zur Erzielung unternehmerischen

533 In diesem Sinne stellt auch PETTINGER fest: ,Many definitions of what management is exist.“ (Pettinger, R. (1994), S. 1).

534 Vgl. Fayol, H. (1917), S. 11.

535 Vgl. Koontz, H. / O’Donnell, C. / Weihrich, H. (1984), S. 64-65.

${ }^{536}$ Vgl. Gulick, L. H. (1937), S. 13. Darüber hinaus ist insbesondere im Kontext der St. Galler Schule auch eine funktionale Differenzierung in Entwickeln, Gestalten und Lenken gebräuchlich. Vgl. hierzu Bleicher, K. / Abegglen, C. (2017), S. 136; Malik, F. (2013), S. 111; Ulrich, H. / Probst, G. J. B. (1995), S. 270-276; Ulrich, H. (1984), S. 113-122. Ebenfalls verbreitet ist eine Dreiteilung in Planung, Steuerung bzw. Organisation (funktional) und Kontrolle. Vgl. z. B. Schauer, R. (2019), S. 12; Hagenloch, T. (2018), S. 68; Mroß, M. (2015), S. 153; Hungenberg, H. (2014), S. 47; Heuermann, R. / Tomenendal, M. (2011), S. 92; Palupski, R. (2002), S. 74. Dies korrespondiert wiederum mit dem Managementzyklus als kybernetischem Prozess. Vgl. z. B. Schierenbeck, H. / Wöhle, C. B. (2016), S. 115; Rathe, A. W. / Irani, D. (1972), S. 26 sowie mit Controllingbezug auch Baum, H.-G. / Coenenberg, A. G. / Günther, T. (2013), S. 7.

${ }^{537} \mathrm{Vgl}$. hierzu auch die Ausführungen in Abschnitt 4.5.1.

538 Vgl. z. B. Oehlrich, M. / Dahmen, A. (2019), S. 42; Preuß, N. / Schöne, L. B. (2016), S. 80-81; Schierenbeck, H. / Wöhle, C. B. (2016), S. 150; Hungenberg, H. (2014), S. 4347; Seeger, B. (2014), S. 49-51; Matzler, K. / Müller, J. (2013), S. 12; Reinspach, R. (2011), S. 85-86; Schuh, G. et al. (2011), S. 367; Meierbeck, R. (2010), S. 299; Hansmann, K.-W. (2006), S. 24; Palupski, R. (2002), S. 83. 
Erfolgs (Wertschöpfung). Eine solche Zweiteilung, obschon nach wie vor terminologisch verbreitet, erscheint jedoch als $\mathrm{zu}$ restriktiv. So würde in Bezug auf das zuvor aufgezeigte dreistufige Zielsystem ein rein strategisch-operatives Management zum einen die normativen Grundlagen des Handelns vernachlässigen, zum anderen gilt für die freiheitliche Gesellschaft, wie auch einleitend herausgearbeitet wurde, dass noch nicht jedes strategische wie auch operative Handeln bereits selbstverständlich gesellschaftlich hinreichend begründet ist. Diese Überlegungen führten insbesondere die St. Galler Schule zur Notwendigkeit der Konzeption eines eigenständigen normativen Managements, welches die strategische und operative Ebene in einen normativen Rahmen einbettet, ${ }^{539}$ allerdings jedoch ohne dieses zur Begründung von Unternehmensethik fruchtbar zu machen. Die verstärkte konzeptionelle Ausarbeitung einer strukturierten normativen Reflexion unternehmerischen Handelns als genuine Unternehmensethik erscheint gerade vor dem immer noch vorherrschenden betriebswirtschaftlichen Fokus auf strategische wie auch operative Fragestellungen in der Forschung, aber auch der unternehmerischen Praxis, welche normativen Fragestellungen ebenfalls häufig noch skeptisch gegenübertritt, hochrelevant. So wird die Beschäftigung mit normativen Problemstellungen vielfach noch als ,,,schöne Idee“, ,Kuschelkapitalismus', ,Sozialromantik' oder , Thema für Sonntagspredigten angesehen, das mit der ,harten unternehmerischen Realität" wenig zu tun habe. ${ }^{* 540}$ Die normative Geschäftsgrundlage der unternehmerischen Tätigkeit wird damit nach wie vor häufig konzeptionell aber auch praktisch vernachlässigt.

Dabei ist $\mathrm{zu}$ betonen, dass sich ein solches normatives Management gerade nicht in einem reinen Complianceansatz ${ }^{541}$ erschöpfen darf, sind doch die

${ }^{539}$ Vgl. Rüegg-Stürm, J. / Grand, S. (2020), S. 75-77; Bleicher, K. / Abegglen, C. (2017), S. 58-62 und S. 199; Rüegg-Stürm, J. / Grand, S. (2017), S. 180-188; Bleicher, K. (2009), S. 154-156; Ulrich, P. / Fluri, E. (1995), S. 18-22; Ulrich, H. / Probst, G. J. B. (1995), S. 282; Ulrich, H. (1984), S. 329-332 sowie insbesondere Ulrich, H. (1981), S. 11-15, welcher nach ULRICH und FLURI erstmalig den Begriff des normativen Managements gebrauchte (vgl. Ulrich, P. / Fluri, E. (1995), S. 21). Vgl. zudem die Ausführungen in Reber, M. / Pifko, C. (2018), S. 70-79; Hutzschenreuter, T. (2015), S. 385; Paul, J. (2015), S. 172. Nach ULRICH und FLURI geht es auf der normativen Ebene des Managements „,um die Wertfragen unternehmerischen Handelns, oder genauer um die angemessene Bewältigung von unternehmungspolitischen Wert- und Interessenkonflikten." (Ulrich, P. / Fluri, E. (1995), S. 21, in der Quelle ebenfalls herv.).

${ }^{540}$ Lingnau, V. / Fuchs, F. (2021), S. 2.

${ }^{541}$ Vgl. z. B. Fissenewert, P. / Wendt, M. (2019); Rick, S. (2018); Hein, R. (2016); Moosmayer, K. (2015); Schwarzbartl, M. / Pyrcek, A. (2013); Steß1, A. (2012). ComplianceManagementansätze stammen ursprünglich aus dem US-amerikanischen Raum mit dem Fokus als Sanktionsvermeidungsstrategie (vgl. z. B. Ulrich, P. / Kaiser, M. (2001), S. 26), 
rechtliche und moralische Sphäre (trotz Überschneidungen) i. d. R. nicht vollständig deckungsgleich. ${ }^{542}$ So laufen auch Vorschläge des reinen ComplianceManagements in die Leere, werden nicht noch zusätzlich die gesellschaftlichen Moralvorstellungen in unternehmerischen Entscheidungen und Handlungen integriert. Es sei daher nochmals betont: „Compliance is not enough!" 543 Auch SHAMOO und RESNIK heben in diesem Sinne hervor: „However, from an ethical point of view, mere compliance is not enough: one must go beyond compliance. Focusing on compliance is a minimalist approach to ethics." ${ }^{\text {"544 }}$ In ähnlicher Weise betonen KAYES, STIRLING und NIELSEN: „Ethical lapses by employees can put organizations at substantial risk. Although improved compliance procedures can help limit this risk, successful efforts must extend beyond compliance [...]. “545 Ebenso resümieren SCHAFFER, AguSTI und DHOOGE: „[T] he law is a floor above which our ethical conduct should rise." ${ }^{\text {"546 }}$ Analog konstatieren auch FREEMAN, HARRISON und WICKS: „Many companies simply don't go far enough when they articulate their ethics policies in terms of compliance with a set of regulations or a code of conduct [...]. “547 Schließlich stellen ebenfalls PIES, HIELSCHER und BECKMANN fest, dass sich die ,gesellschaftliche Akzeptanz [..] zunehmend als Engpass [erweist] [...], denn längst schon wird nicht mehr alles, was legal ist, auch als legitim anerkannt."548 Folglich muss das normative Management insbesondere auch beruhend auf gesellschaftlich anerkannten,

insbesondere auch vor dem Hintergrund einer verschärften Gesetzgebung, z. B. durch die Federal Sentencing Guidelines.

542 Vgl. hierzu die Ausführungen in Abschnitt 2.5.1.

543 Lingnau, V. / Fuchs, F. (2021), S. 11. Vgl. auch Kennedy-Glans, D. / Schulz, R. (2005), S. XIX; Wheeler, W. A. (1992), S. 10. Vgl. in diesem Kontext auch Perrin, I. (2010), S. 13.

544 Shamoo, A. E. / Resnik, D. B. (2006), S. 374. So hebt auch SuNDRUM die Bedeutung von ,[m]oving beyond compliance and control [...]“ (Sundrum, E. (2004), S. 192) hervor.

545 Kayes, D. C. / Stirling, D. / Nielsen, T. M. (2007), S. 61. In diesem Zusammenhang wird häufig auf Paine, L. S. (1994) rekurriert und die Bedeutung von Integrität hervorgehoben. Vgl. auch Steinmann, H. / Olbrich, T. (1998), S. 97-99 sowie die detaillierten Überlegungen in Lingnau, V. / Fuchs, F. / Beham, F. (2018). Zum Begriff der Integrität vgl. auch die Ausführungen in Abschnitt 4.7.

546 Schaffer, R. / Agusti, F. / Dhooge, L. J. (2018), S. 44.

${ }^{547}$ Freeman, R. E. / Harrison, J. S. / Wicks, A. C. (2007), S. 101.

548 Pies, I. / Hielscher, S. / Beckmann, M. (2009a), S. 325. In ähnlicher Weise konstatiert auch BESCHORNER: „Unternehmen sehen sich von zahlreichen gesellschaftlichen Gruppen mit Erwartungen und Ansprüchen konfrontiert. Über wirtschaftliches und legales Agieren hinaus wird von diesen Gruppen zunehmend ein ethisch legitimierbares Unternehmensverhalten gefordert.“ (Beschorner, T. (2004), S. 256). Aus unternehmerischer Perspektive stellen 
guten Gründen ${ }^{549}$ die gewählten Strategien und operativen Entscheidungen bzw. Handlungen überzeugend begründen können, welches somit aus ethischer Perspektive zum Kern dieser Managementfunktion erklärt werden kann. So stellt auch RUEGG-STURM in Bezug auf die Stakeholderansprüche fest, dass diese „einer respektvollen Würdigung und einer sorgfältigen argumentativen Abwägung [bedürfen] [.] und die getroffene Entscheidung [.] schliesslich nachvollziehbar zu begründen (Legitimierung) [ist]. “550

Die vorausgegangenen grundlegenden funktionalen Managementüberlegungen können nun wiederum mit dem institutionellen Managementbegriff in Verbindung gebracht werden. Dabei gilt in einem ersten Schritt, dass aus institutioneller Perspektive prinzipiell zwar jede Managementebene zu einem gewissen Grad mit normativen, strategischen und operativen Entscheidungen befasst ist. ${ }^{551}$ Allerdings kann, wie auch die betriebliche Praxis zeigt, durchaus eine schwerpunktmäßige Zuordnung der Tätigkeiten vorgenommen werden. So werden normative und eher langfristige bzw. weniger konkretisierte Entscheidungen in der Regel vom Topmanagement getroffen, welches auch als „Corporate Figurehead“ nach außen wie innen das Unternehmen maßgeblich repräsentiert bzw. der zentrale Adressat für die normative Rahmengestaltung im Unternehmen darstellt. Das mittlere Management nimmt dagegen regelmäßig vornehmlich Aufgaben und Entscheidungen mittlerer Reichweite und Konkretisiertheit sowie mit strategischem Bezug wahr, während das untere Management eher mit sehr konkreten bzw. operativen Entscheidungen betraut ist. Diese Zusammenhänge fasst die nachfolgende Abbildung 4.16 zusammen.

zudem ULRICH und KAISER fest: „Die mitunter sich schnell wandelnden Legitimitätsansprüche bilden aber für die öffentliche Beurteilung von Unternehmen oft die wichtigere Referenzgrösse als die konkreten Inhalte des kodifizierten Rechts. Vor diesem Hintergrund bieten bloss legalitätsorientierte Compliance-Konzepte keinen zuverlässigen Schutz vor öffentlichen Legitimitätsanfechtungen.“ (Ulrich, P. / Kaiser, M. (2001), S. 27).

${ }^{549}$ In diesem Sinne konstatieren auch STEINMANN und LÖHR: „Das Erfordernis ,guter Gründe" impliziert bereits eine wichtige Unterscheidung, nämlich zwischen Legitimität und Legalität.“ (Steinmann, H. / Löhr, A. (1997), S. 12, in der Quelle ebenfalls herv.). Ebenso betonen auch ULRICH und KAISER die Relevanz, „das unternehmerische Wirken vor der immer kritischeren Öffentlichkeit mit guten Gründen rechtfertigen zu können.“ (Ulrich, P. / Kaiser, M. (2001), S. 29).

${ }^{550}$ Rüegg-Stürm, J. (2003), S. 33.

551 So stellen auch BLEICHER und ABEGGLEN fest: „Eine Führungskraft kann (und vermehrt auch: sollte) institutionell in der Organisation zugleich normative wie strategische Funktionen wahrnehmen und um deren operative Durchsetzung bemüht sein." (Bleicher, K. / Abegglen, C. (2017), S. 458). 


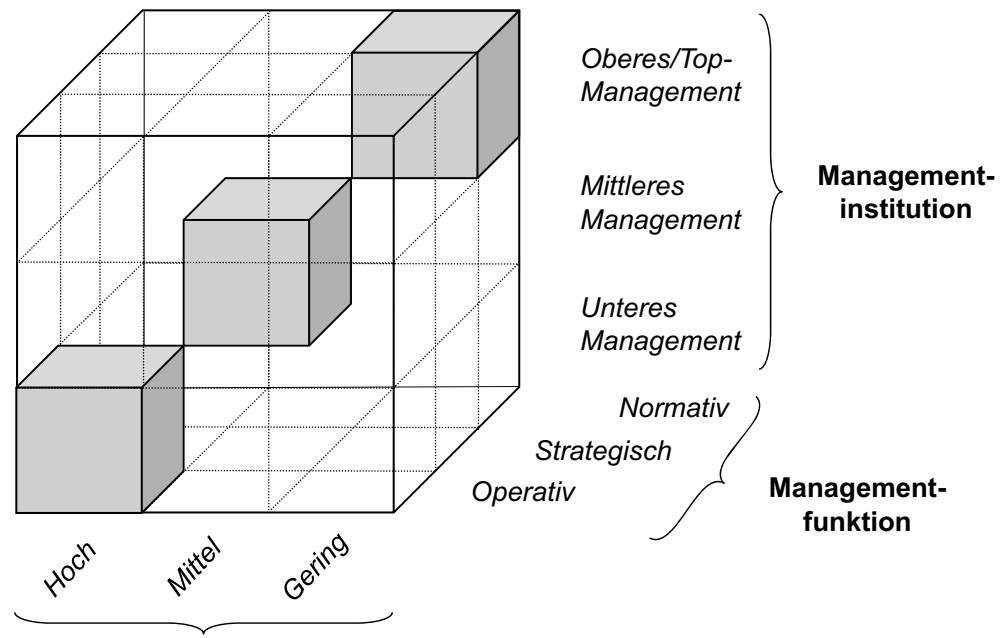

Konkretisiertheit der betrachteten Ziele/Ansprüche

Abbildung 4.16 Zusammenführung von funktionaler und institutioneller Managementebene sowie zeitlicher Bezug

Die bisherigen Ausführungen dieses Kapitels können nun nochmals subsumierend in der folgenden Tabelle 4.4 dargestellt werden, welche neben der jeweiligen Managementfunktion die korrespondierende institutionelle Managementebene, die dominante unternehmerische Zielebene sowie die hieraus primär abgeleiteten Ansprüche umfasst. Dabei könnte darüber hinaus und in Anlehnung an die bekannte Klassifikation der St. Galler Schule ${ }^{552}$ die normative Ebene auch als Begründungsebene, die strategische Ebene als Gestaltungsebene und die operative Ebene als Nutzungsebene der unternehmerischen Wertschöpfungspotentiale bezeichnet werden.

Die vorausgegangenen Überlegungen haben sowohl in Bezug auf das unternehmerische Zielsystem wie auch im Managementkontext die Bedeutung einer kritischen normativen Reflexion aufgezeigt. Diese werden im Weiteren explizit zur Begründung einer Unternehmensethik herangezogen. Es gilt folglich: Unternehmensethik (im praktischen Sinne) dient als normatives Management (funktional) der Sicherstellung einer jederzeit hinreichenden gesellschaftlichen Legitimität als

552 Vgl. hierzu nochmals die Ausführungen in Fn. 536. 
Tabelle 4.4 Synopse von unternehmerischer Managementfunktion, -institution und den korrespondierenden Zielen und Ansprüchen

\begin{tabular}{|l|l|l|l|}
$\begin{array}{l}\text { Management- } \\
\text { funktion }\end{array}$ & $\begin{array}{l}\text { Primäre } \\
\text { Managementebene }\end{array}$ & $\begin{array}{l}\text { Korrespondierender } \\
\text { Zielinhalt }\end{array}$ & Anspruchsart \\
\hline $\begin{array}{l}\text { Normativ } \\
\text { („Begründen“) }\end{array}$ & Oberes Management & $\begin{array}{l}\text { Aufbau und Erhalt von } \\
\text { Legitimität }\end{array}$ & Organisationsinteresse \\
\hline $\begin{array}{l}\text { Strategisch } \\
\text { („Gestalten“) }\end{array}$ & Mittleres Management & $\begin{array}{l}\text { Aufbau und Erhalt von } \\
\text { Erfolgs- bzw. } \\
\text { Wertschöpfungs- } \\
\text { potentialen }\end{array}$ & Organisationsanliegen \\
\hline $\begin{array}{l}\text { Operativ } \\
\text { („Nutzen“) }\end{array}$ & Unteres Management & $\begin{array}{l}\text { Effektive und effiziente } \\
\text { Nutzung von Erfolgs- } \\
\text { bzw. Wertschöpfungs- } \\
\text { potentialen }\end{array}$ & $\begin{array}{l}\text { Organisations- } \\
\text { forderungen }\end{array}$ \\
\hline
\end{tabular}

Grundvoraussetzung dauerhaft erfolgreichen unternehmerischen Handelns und bedarf der Institutionalisierung sowie der systematischen Methoden.

Der Begriff der Legitimität steht damit im Zentrum der unternehmensethischen Konzeption dieser Arbeit. Jedoch zeigt auch dieser eine große Bandbreite an begrifflicher Ausdeutung, weshalb der Legitimitätsbegriff im nachfolgenden Kapitel nochmals detaillierter konzeptionell eruiert und für die weiteren Ausführungen dieser Arbeit fruchtbar gemacht wird.

\subsubsection{Zum Begriff der Legitimität und deren Implikationen für das normative Management}

Befasst man sich näher mit der Historie des Wortes Legitimität, so zeigt sich interessanterweise mit dem Ursprung im lateinischen „lex“ bzw. „legis“ zuerst eine gewisse Nähe zum Terminus der Legalität, welcher etymologisch eine vergleichbare Wurzel aufweist. ${ }^{553}$ Inhaltlich kann jedoch klar festgestellt werden, dass beide Konzepte deutlich unterschiedliche Konstrukte repräsentieren. So stellt auch GOBBEL pointiert fest: „Legalität und Legitimität sind [.] nicht deckungsgleich. “554 Auch nach BoulDING gilt in diesem Sinne: „The two concepts are not

553 Vgl. Baur, S.C. (2018), S. 30; Engelhardt, B. v. (2016), S. 32-33; Lamb, R. D. (2014), S. 15; Glaser, K. (2013), S. 37; Foldvary, F. E. (2011), S. 724; Perrin, I. (2010), S. 13; Mulligan, S. P. (2006), S. 356-357; Schliesky, U. (2004), S. 181-182; Zelditch, M. (2001), S. 33.

554 Göbel, E. (2020), S. 152. 
the same." ${ }^{555}$ Während Legalität das Konstrukt der rechtlichen Sphäre beschreibt, referenziert der Begriff der Legitimität im wirtschaftsethischen Sinne ${ }^{556}$ auf die Moral. Da, wie gezeigt wurde, Recht und Moral im besten Falle überlappen, jedoch nicht identisch sind ${ }^{557}$, ergibt sich vor dem Hintergrund des Nichtausreichens einer legalistischen Betrachtung auch hier nochmals die Relevanz einer klaren begrifflichen Differenzierung. ${ }^{558}$

Wissenschaftshistorisch wird dabei der erste explizit wissenschaftliche Gebrauch des Legitimitätsbegriffs häufig MAX WEBER zugeschrieben ${ }^{559}$, welcher den Begriff in seinem Werk „Wirtschaft und Gesellschaft“ zur Analyse der Anerkennung von Herrschaftsordnungen gebrauchte, wobei er jedoch keine detaillierte Definition des Begriffs vornahm. Vielmehr untersuchte er, basierend auf einem eher intuitiv vorausgesetzten Begriffsverständnis ${ }^{560}$, ein soziales Handeln, welches an einer ,legitimen Ordnung ${ }^{\prime 561}$ orientiert ist, welchem er wiederum die größte Stabilität bzw. Verbindlichkeit zurechnete. ${ }^{562}$ An diese grundlegenden Überlegungen WEBERS schlossen sich in der sozialwissenschaftlichen Forschung nun einige Autoren, wie z. B. PARSONS ${ }^{563}$, an, welcher zu den ersten Autoren zählt, die nun auch eine explizite Definition des Begriffs vorgenommen haben. Neben anderen Autoren wie BOULDING ${ }^{564}$ hat dann auch PARSONS

555 Boulding, K. E. (1974), S. 512.

556 Darüber hinaus existieren in anderen Disziplinen auch weitere Begriffsauffassungen, welche im Rahmen dieser Arbeit jedoch ausgeklammert seien. Vgl. hierzu Glaser, K. (2013), S. 22-26 sowie zur Bandbreite an existierenden Begriffsverwendungen auch Mulligan, S. P. (2006), S. 367.

557 So stellen auch DowLING und PFEFFER fest: ,Though in a democratic polity laws are likely to be correlated with societal norms and values, their correlations are less than perfect.“ (Dowling, J. / Pfeffer, J. (1975), S. 124). Darüber hinaus merkt BEETHAM mit einem Metafokus an: „On its own, legal validity is insufficient to secure legitimacy, since the rules through which power is acquired and exercised themselves stand in need of justification." (Beetham, D. (2013), S. 17).

558 Vgl. hierzu nochmals detailliert Abschnitt 2.5.1.

${ }^{559}$ Vgl. Deephouse, D. L. et al. (2017), S. 30; Gehman, J. / Lefsrud, L. M. / Fast, S. (2017), S. 301; Ruef, M. / Scott, W. R. (1998), S. 877. Zu einer detaillierten historischen Analyse des Legitimitätsbegriffs vgl. auch Tuschke, A. (2005), S. 35-40.

560 Vgl. hierzu auch Scherm, E. / Pietsch, G. (2007), S. 16.

561 Weber, M. (1976), S. 16, in der Quelle gesperrt herv.

562 Vgl. Weber, M. (1976), S. 16.

${ }^{563}$ Vgl. Parsons, T. (1965), S. 172-177. Vgl. auch Parsons, T. (1971), S. 14-15; Parsons, T. (1968), S. 81-83.

564 Vgl. Boulding, K. E. (1974), S. 511-512. 
Schüler LUHMANN ${ }^{565}$ den Legitimitätsbegriff definiert und in seinen Werken gebraucht. Interessanterweise lässt sich im modernen Schrifttum gerade auch in der politikwissenschaftlichen Forschung mittlerweile eine starke Rezeption des Legitimitätsbegriffs konstatieren. ${ }^{566}$ Darüber hinaus existiert auch seit Ende der 1970er Jahren im angelsächsischen Raum ein Transfer der Überlegungen auf die organisationale Ebene, welches mit dem Begriff des Neoinstitutionalismus korrespondiert. ${ }^{567}$ Mit dem Entstehen der St. Galler Schule hat schließlich als jüngste Entwicklung der Begriff der Legitimität auch zunehmend Einzug in das deutschsprachige betriebswirtschaftliche sowie insbesondere wirtschaftsethische Schrifttum genommen, womit sich der Fokus von der Begründetheit gesellschaftlicher Herrschaftsstrukturen auf die gesellschaftliche Anerkennung von Betrieben als legitimierungsbedürftigen Einheiten verschiebt. ${ }^{568}$

Zur näheren inhaltlichen Bestimmung des Legitimitätsbegriffs hat die Forschung insbesondere ab den 1970er Jahren eine Fülle an Definitionsvorschlägen erarbeitet. Neben den soziologischen Überlegungen von PARSONS, BERGER und LUCKMANN $^{569}$ sowie LUHMANN findet sich so z. B. bei MAURER die Auffassung von Legitimität als organisationalem ,right to exist“"570. DOWLING und PFEFFER definierten dabei wenig später Legitimität als „,congruence between the social

\footnotetext{
565 Vgl. Luhmann, N. (1975), S. 27-37.

566 Vgl. z. B. die Ausführungen in Engelhardt, B. v. (2016); Kemper, U. (2015); Stübig, S. (2015); Voigt, R. (2015); Fehr, H. (2014); Glaser, K. (2013); Akude, J. E. / Daun, A. / Egner, D. (2011); Bürgler, B. (2009); Steber, M. (2008); Schliesky, U. (2004).

${ }^{567}$ Diese Forschung fokussiert jedoch mit dem Zentralbegriff der Organisation lediglich auf soziale Einheiten. Da jedoch realiter im Wirtschaftskontext der Mesoebene i. d. R. soziotechnische Ganzheiten gesellschaftlich evaluiert werden, ist hier zwischen dem betrieblichen Evaluationsobjekt und der Verantwortung tragenden Instanz „Organisation“ zu unterscheiden. Vgl. hierzu und insbesondere auch zur Relevanz der Legitimierung aus neoinstitutionalistischer Perspektive Abschnitt 4.4.3.1.

568 Vgl. Bleicher, K. / Abegglen, C. (2017), S. 282; Ulrich, P. (2008b), S. 335; Ulrich, P. (2007), S. 5; Rüegg-Stürm, J. (2003), S. 33.

569 Bei diesen Autoren findet sich allerdings nur eine indirekte Definition des Legitimitätsbegriffs, da diese vielmehr auf den prozessualen Aspekt der Legitimierung verweisen. So definiert etwa PARSONS Legitimierung als ,, appraisal of action in terms of shared or common values in the context of the involvement of the action in the social system." (Parsons, T. (1965), S. 175, in der Quelle ebenfalls herv.). BERGER und LUCKMANN definieren dagegen: „Legitimation ,explains" the institutional order by ascribing cognitive validity to its objectivated meanings.“ (Berger, P. L. / Luckmann, T. (1967), S. 93).

${ }^{570}$ Maurer, J. G. (1971), S. 361.
} 
values associated with or implied by [organizational] activities and the norms of acceptable behavior in the larger social system of which they are a part.“571 Ähnlich erachtet auch RICHARDSON Legitimität als ,a quality of congruence between acts and social values." ${ }^{\text {"572 }}$ ASHFORTH und GIBBS wiederum referieren hinsichtlich des Status einer legitimierten Einheit auf ,the extend that its means and ends appear to conform with social norms, values, and expectations. “573 Mitte der 1990er Jahre entwickelte schließlich SuchMAN einen Vorschlag, welcher bis heute, trotz aller Kritik und Weiterentwicklung in Bezug auf zunehmend komplexere und tendenziell enumerative Ansätze ${ }^{574}$ als Standarddefinition der Legitimität gelten kann. ${ }^{575}$ In diesem Sinne definiert SUCHMAN Legitimität als eine ,generalized perception or assumption that the actions of an entity are desirable, proper, or appropriate within some socially constructed system of norms, values, beliefs, and definitions." ${ }^{\text {"576 }}$ Einen Überblick über existierende Legitimitätsdefinitionen gibt auch nochmals die nachfolgende Tabelle 4.5.

${ }^{571}$ Dowling, J. / Pfeffer, J. (1975), S. 122.

572 Richardson, A. J. (1985), S. 140.

573 Ashforth, B. E. / Gibbs, B. W. (1990), S. 177.

${ }^{574}$ Diese Vorgehensweise schlägt auch BITEKTINE vor, vgl. hierzu Bitektine, A. (2011), S. 153.

575 So betonen DEEPHOUSE ET AL.: ,Overall, most research in the last two decades has, in some way, followed Suchman's [..] definition of legitimacy, evident by the many citations not only in management journals but in journals from other disciplines and languages." (Deephouse, D. L. et al. (2017), S. 32). Auch Gehman, LEFSRUd und FAST betonen in diesem Sinne, dass SUCHMAN ,offered what has become perhaps the most widely cited definition of legitimacy.“ (Gehman, J. / Lefsrud, L. M. / Fast, S. (2017), S. 301-302). Vgl. auch Bitektine, A. / Haack, P. (2015), S. 50; Koch, S. (2009), S. 126; Stelzer, F. (2008), S. 7; Ruef, M. / Scott, W. R. (1998), S. 878.

576 Suchman, M. C. (1995), S. 574. 
Tabelle 4.5 Überblick über existierende Legitimitätsdefinitionen

Autor

Maurer, J. G. (1971), S. 361

Dowling, J. / Pfeffer, J.

(1975), S. 122

Luhmann, N. (1975), S. 28

Hannan, M. T. / Freeman, J. (1977), S. 932

Meyer, J. W. / Rowan, B. (1977), S. 351

Pfeffer, J. (1981), S. 4

Richardson, A. J. (1985), S. 140

Ashforth, B. E. / Gibbs, B. W. (1990), S. 177

Oliver, C. (1991), S. 160

Elsbach, K. D. / Sutton, R. I.

(1992), S. 700

Hannan, M. T. / Carroll, G. R. (1992), S. 33

Meyer, J. W. / Scott, W. R. (1992), S. 201

Aldrich, H. E. / Fiol, C. M. (1994), S. 645-646

Rao, H. (1994), S. 31

Preston, A. M. et al. (1995), S. $\mathbf{5 1 0}$

Suchman, M. C. (1995), S. 574

Deephouse, D. L. (1996), S. 1025

Tyler, T. R. (1997), S. 323

Kostova, T. / Zaheer, S.

(1999), S. 64

Marnoch, G. / McKee, L. /

Dinnie, N. (2000), S. 972

\section{Definition}

„right to exist"

"congruence between the social values associated with or implied by [organizational] activities and the norms of acceptable behavior in the larger social system of which they are a part."

„Man kann Legitimität auffassen als eine generalisierte Bereitschaft, inhaltlich noch unbestimmte Entscheidungen innerhalb gewisser Toleranzgrenzen hinzunehmen." (in der Quelle ebenfalls herv.)

„Any legitimacy an organization has been able to generate constitutes an asset in manipulating the environment. To the extent that adaptation [...] violates the legitimacy claims, it incurs considerable costs."

„legitimacy [...] protects organizations from immediate sanctions for variations in technical performance [...]."

„Activities which are accepted and expected within a context are then said to be legitimate within that context."

"Legitimacy is defined as a quality of congruence between acts and social values."

„An organization is said to be legitimate to the extent that its means and ends appear to conform with social norms, values, and expectations [...].“ „social fitness“

"Legitimacy is conferred when stakeholders - that is, internal and external audiences affected by organizational outcomes - endorse and support an organization's goals and activities. And an organization can be described as legitimate when it is endorsed and supported by a segment of society large enough to ensure its effectiveness and survival [...]."

„a form receives [legitimacy] ${ }^{\mathrm{a}}$ to the extent that its structure and routines follow the dictates of the prevailing institutional rules."

„organizational legitimacy refers to the degree of cultural support for an organization - the extent to which the array of established cultural accounts provide explanations for its existence, functioning, and jurisdiction, and lack or deny alternatives."

„a) how taken for granted a new form is and b) the extent to which a new form conforms to recognized principles or accepted rules and standards."

„an entity as desirable, proper, and appropriate in a widely shared system of beliefs and norms [...]."

"Legitimacy is conceived as congruence between institutional actions and social values [...]."

„Legitimacy is a generalized perception or assumption that the actions of an entity are desirable, proper, or appropriate within some socially constructed system of norms, values, beliefs, and definitions." (in der Quelle zusätzlich fett herv.)

„Organizational legitimacy [...] is defined as a status conferred by social actors. [...] [A] legitimate organization is one whose values and actions are congruent with that social actor's values and expectations for action [...]. [L]egitimacy is the endorsement of an organization by social actors [...]."

"the belief that authorities are entitled to be obeyed [...]."

„the acceptance of the organization by its environment [...] vital for organizational survival and success [...]."

„activities are perceived as ,desirable, proper and appropriate' within the prevailing context of society [...]." 


\section{Tabelle 4.5 (Fortsetzung)}

Zelditch, M. (2001), S. 33

Zimmerman, M. A. / Zeitz, G. J. (2002), S. 414

Pfeffer, J. / Salancik, G. R. (2003), S. 193

Delmar, F. / Shane, S. (2004), S. 388

Núñez Nickel, M. / Moyano Fuentes, J. (2004), S. 43

Deephouse, D. L. / Carter, S. M. (2005), S. 332

Washington, M. / Zajac, E. J. (2005), S. 284

Rindova, V. P. / Pollock, T. G. / Hayward, M. L. A.

(2006), S. 55

Brink, S. (2009), S. 91

Lamb, R. D. (2014), S. 15

Scott, W. R. (2014), S. 72

Melé, D. / Armengou, J. (2016), S. 730

Koch, S. (2018), S. 202 „something is legitimate if it is in accord with the norms, values, beliefs, practices, and procedures accepted by a group."

„Legitimacy, a social judgment of acceptance, appropriateness, and desirability, enables organizations to access other resources needed to survive and grow."

"Legitimacy is bound up with social norms and values; and while it is not correlated perfectly with either law or economic viability, it bears some relationship to both."

„the legitimacy of a new venture is the extent to which people perceive that it adheres to accepted principles, rules, norms, standards, and ways of doing things [...]."

„A form gains [legitimacy] ${ }^{\mathrm{b}}$ when it attains a socially taken-for-granted character [...]."

„we view legitimacy as the social acceptance resulting from adherence to regulative, normative or cognitive norms and expectations."

"Legitimacy refers to the level of social acceptability bestowed upon a set of activities or actors [...]."

"Legitimacy is the degree to which broader publics view a company's activities as socially acceptable and desirable because its practices comply with industry norms and broader societal expectations [...]."

„Ein Unternehmen kann [.] dann als legitim eingestuft werden, wenn sowohl das Unternehmen als auch dessen Ziele und Mittel zur Zielerreichung mit den von relevanten Anspruchsgruppen vertretenen, allgemein anerkannten gesellschaftlichen Werten und Normen übereinstimmen und das Unternehmen entsprechend als wünschenswert, angemessen und passend wahrgenommen wird." (in der Quelle ebenfalls herv.)

„To claim that something is legitimate is to give a moral or normative reason (,it is right') to obey, support, accept, imitate, comply with, or refrain from opposing it within some bounded range of activity or experience."

„legitimacy is [...] a condition reflecting perceived consonance with relevant rules and laws or normative values, or alignment with cultural-cognitive frameworks."

„the moral legitimacy of a business or of a particular project or activity is based on sound ethical principles."

„Der Begriff ,organisationale Legitimität' bezeichnet den Grad der Anerkennung, den die Bedeutung(en) der Existenz, der spezifischen Verfasstheit bzw. des jeweiligen Handelns einer Organisation innerhalb einer gemeinsamen institutionellen (Bedeutungs-)Umwelt durch weitere soziale Akteure zugeschrieben bekommt und somit zu einem spezifischen Zeitpunkt auf sich vereint."

an der Originalquelle ,legitimation“ im Sinne des Legitimitätsbegriffs.

bIn der Originalquelle ,legitimation“ im Sinne des Legitimitätsbegriffs.

Den meisten Definitionen ist dabei, neben kleineren Unterschieden, der Korrespondenzgedanke von Handlungen oder Konsequenzen mit gesellschaftlich existierenden Werten gemein, weshalb diese Ansätze klar als empirisch ausgerichtet aufgefasst werden können und folglich auf einem deskriptiv-ethischen Fundament aufbauen. Neben diesem nach wie vor vorherrschenden, deskriptiven 
bzw. empirischen Ansatz haben neuerdings, basierend auf den Überlegungen von FOLDVARY ${ }^{577}$ sowie BAUR und PALAZZO ${ }^{578}$, MElÉ und ARMENGOU ein Legitimitätskonzept vorgeschlagen ${ }^{579}$, welches nicht mit dem faktisch vorherrschenden Moralbestand beginnt, sondern auf ethischen Prinzipien beruht und klar einer ethisch-normativen Auffassung folgt. ${ }^{580}$ Die vorausgegangenen Überlegungen können dabei auch als fruchtbares Fundament der Unternehmensethikkonzeption dieser Arbeit herangezogen werden. So folgt, wie im Weiteren noch ausgeführt wird, die Arbeit zwar grundsätzlich der empirischen Auffassung Suchmans im Sinne eines deskriptiv-ethischen bzw. moralbasierten Fundaments der Legitimität, wird jedoch im Rahmen eines zweistufigen Ansatzes zusätzlich neben einer kritisch-deskriptiven Perspektive auch die Möglichkeit einer ethisch-normativen Evaluation der faktisch herrschenden Legitimitätsnormen offerieren. ${ }^{581}$

Darüber hinaus hat die Literatur insbesondere seit Mitte der 1990er Jahre mit dem Beitrag von ALDRICH und FIOL noch weitere Systematisierungsansätze hervorgebracht, welche sich auf eine genauere Spezifikation der Legitimität als multidimensionales Konzept fokussieren. So unterscheiden ALDRICH und FIOL eine kognitive und eine soziopolitische Dimension der Legitimität, wobei die erstere auf das Verständnis z. B. gegenüber einem Produkt oder einer Handlung abzielt (im Extremfall: „Selbstverständlichkeit“ als Legitimierungsbasis), während letztere die gesellschaftlichen Basiswerte repräsentieren, wie sie auch von zentralen, einflussreichen Stakeholdern bzw. Stakeholdergruppen vertreten werden. ${ }^{582}$ Hieran anknüpfend hat sich eine Vielzahl an konzeptionellen Erweiterungen und Abänderungen entwickelt. So unterscheidet die ebenfalls häufig aufgegriffene Systematik nach SUCHMAN neben der kognitiven Komponente der Legitimität auch eine pragmatische und moralische Form, wobei die pragmatische Legitimität durch Eigennutzüberlegungen fundiert sei (z. B. Nützlichkeit eines Unternehmens), während die moralische Ausprägung eine allgemeine normative Zustimmung repräsentiere. Die kognitive Komponente wird von SUCHMAN dann wiederum im Sinne von selbstverständlichen Werten gedeutet, wobei sich

\footnotetext{
577 Vgl. Foldvary, F. E. (2011), S. 724-725.

578 Vgl. Baur, D. / Palazzo, G. (2011), S. 584-585.

${ }^{579}$ Dabei erscheint jedoch gerade die von den Autoren gewählte Bezeichnung als ,,moral legitimacy“ irreführend, da diese begrifflich über die Moral einen stark deskriptiven Fokus impliziert. Besser wäre in diesem Zusammenhang von einer „ethical-“ oder „,ethically justified legitimacy" zu sprechen.

${ }^{580}$ Vgl. Melé, D. / Armengou, J. (2016), S. 730. Vgl. auch Bowen, F. (2019), S. 262.

${ }^{581} \mathrm{Vgl}$. hierzu detailliert die Ausführungen in Abschnitt 4.5.2.

${ }^{582}$ Vgl. Aldrich, H. E. / Fiol, C. M. (1994), S. 645-646 und S. 648. Vgl. auch Aldrich, H. E. / Baker, T. (2001), S. 211.
} 
zwischen den drei Legitimitätstypen ein absteigendes Maß an organisationaler Beeinflussbarkeit ergibt, welches wiederum invers hierzu mit einer größeren Stabilität dieser Legitimitätsauffassungen korrespondiere. ${ }^{583}$ In weiteren darauffolgenden Veröffentlichungen zeigt sich im Detail jedoch ein heterogenes Bild. ${ }^{584}$ So differenzieren z. B. FOREMAN und WHETTEN ${ }^{585}$ lediglich eine kognitive und pragmatische Dimension, während GoLANT und SILLINCE ${ }^{586}$ eine kognitive und evaluative Legitimität unterscheiden und KOSTOVA und RоTH ${ }^{587}$ eine interne und externe Dimension. ${ }^{588}$ RUEF und SCOTT differenzieren wiederum einerseits die soziopolitische Dimension nochmals in eine normative und regulative Komponente $^{589}$ und stellen diese der kognitiven Legitimität gegenüber, andererseits unterscheiden sie auch zwischen technischer und managementbezogener Legitimität. ${ }^{590}$ Ähnlich konzipieren auch ZIMMERMAN und ZEITZ Legitimität in einer kognitiven, soziopolitisch normativen sowie soziopolitisch regulativen Dimension. ${ }^{591}$ Darüber hinaus findet sich bei DEEPHOUSE ET AL. auch eine viergliedrige Taxonomie in regulatorische, pragmatische, normative sowie kulturell-kognitive

${ }^{583}$ Vgl. Suchman, M. C. (1995), S. 577-585. Diese Klassifikation findet sich auch in einer Reihe weiterer Veröffentlichungen, so z. B. bei Thomas, T. E. / Lamm, E. (2012), S. 193195; Lamberti, L. / Lettieri, E. (2011), S. 464-465; O’Dwyer, B. / Owen, D. / Unerman, J. (2011), S. 33 und S. 36; Kumar, R. / Das, T. K. (2007), S. 1434-1442; Palazzo, G. / Scherer, A. G. (2006), S. 72-73; Johnson, J. / Holub, M. J. (2003), S. 272-273; Zyglidopoulos, S.C. (2003), S. 74; Barron, D. N. (1998), S. 208-209.

584 Vgl. auch den Überblick bei Bitektine, A. (2011), S. 154.

${ }^{585}$ Vgl. Foreman, P. / Whetten, D. A. (2002), S. 623.

${ }^{586}$ Vgl. Golant, B. D. / Sillince, J. A. A. (2007), S. 1149-1150.

${ }^{587}$ Vgl. Kostova, T. / Roth, K. (2002), S. 220-221. Vgl. auch Drori, I. / Honig, B. (2013), S. 345-348; Human, S. E. / Provan, K. G. (2000), S. 328; Kostova, T. / Zaheer, S. (1999), S. 67.

${ }^{588}$ Im politikwissenschaftlichen Kontext findet sich teils auch eine Differenzierung zwischen Input- und Outputlegitimität staatlichen Handelns (vgl. z. B. Scharpf, F. W. (2009), S. 182). Diese könnte im Transfer auch für die organisationale Ebene genutzt werden. In diesem Falle kann jedoch generell nur eine begrenzte Legitimierung über die Inputseite bzw. über Mitbestimmungs- oder Kontrollrechte erfolgen, welche vor allem den Mitarbeitern bzw. im begrenzten Maße auch den Gesellschaftern zukommt, so dass sich Unternehmen gegenüber den meisten Stakeholdern vor allem über ihre Outputs legitimieren müssen.

${ }^{589}$ Vgl. auch Aldrich, H. E. / Ruef, M. / Lippmann, S. (2020), S. 195-197. Die normative Legitimität entspricht dabei im Wesentlichen der ,moralischen Legitimität“ bei SUCHMAN. Vgl. Stelzer, F. (2008), S. 8.

${ }^{590}$ Vgl. Ruef, M. / Scott, W. R. (1998), S. 878-880. Vgl. auch Scott, W. R. (2014), S. 60 und S. 71-75.

${ }^{591}$ Vgl. Zimmerman, M. A. / Zeitz, G. J. (2002), S. 418-420. 
Legitimität. ${ }^{592}$ Schließlich existieren sogar noch deutlich feiner differenzierte Konzeptionen, so z. B. bei BEAULIEU und PASQUERO, die eine legale, identitätsbezogene, moralische, technische, institutionelle sowie wahrnehmungsbezogene Legitimität unterscheiden. ${ }^{593}$ Einen Überblick gibt nochmals die nachfolgende Tabelle 4.6.

Tabelle 4.6 Überblick über existierende Systematiken zu Legitimitätsformen

\begin{tabular}{|c|c|}
\hline Autor & Legitimitätsformen \\
\hline Aldrich, H. E. / Fiol, C. M. (1994), S. 645-646 und S. 648 & cognitive, sociopolitical \\
\hline Suchman, M. C. (1995), S. 577-584 & $\begin{array}{l}\text { cognitive, pragmatic, moral (consequential, procedural, struc- } \\
\text { tural, personal) }\end{array}$ \\
\hline Deephouse, D. L. (1996), S. 1025 & regulatory, public \\
\hline Barron, D. N. (1998), S. 208-209 & cognitive, pragmatic, moral \\
\hline Ruef, M. / Scott, W. R. (1998), S. 878-880 & $\begin{array}{l}\text { 1. cognitive, normative, regulative } \\
\text { 2. managerial, technical }\end{array}$ \\
\hline Kostova, T. / Zaheer, S. (1999), S. 67 & internal, external \\
\hline Human, S. E. / Provan, K. G. (2000), S. 328 & internal, external \\
\hline Aldrich, H. E. / Baker, T. (2001), S. 211 & cognitive, sociopolitical \\
\hline Beaulieu, S. / Pasquero, J. (2002), S. 54 & legal, identity, moral, technical, institutional, perceptual \\
\hline Foreman, P. / Whetten, D. A. (2002), S. 623 & cognitive, pragmatic \\
\hline Kostova, T. / Roth, K. (2002), S. 220-221 & internal, external \\
\hline Zimmerman, M. A. / Zeitz, G. J. (2002), S. 418-420 & cognitive, sociopolitical normative, sociopolitical regulatory \\
\hline Johnson, J. / Holub, M. J. (2003), S. 272-273 & cognitive, pragmatic, moral \\
\hline Zyglidopoulos, S. C. (2003), S. 74 & cognitive, pragmatic, moral \\
\hline Palazzo, G. / Scherer, A. G. (2006), S. 72-73 & cognitive, pragmatic, moral \\
\hline Golant, B. D. / Sillince, J. A. A. (2007), S. 1149-1150 & cognitive, evaluative \\
\hline Kumar, R. / Das, T. K. (2007), S. 1434-1442 & cognitive, pragmatic, moral \\
\hline Scharpf, F. W. (2009), S. 182 & input, output \\
\hline Vergne, J.-P. (2011), S. 487 & environmental, competitive, accountability, transactional \\
\hline O’Dwyer, B. / Owen, D. / Unerman, J. (2011), S. 33-37 & cognitive, pragmatic, moral \\
\hline Thomas, T. E. / Lamm, E. (2012), S. 193-195 & cognitive, pragmatic, moral \\
\hline Scott, W. R. (2014), S. 59-74 & cultural-cognitive, normative, regulative \\
\hline Deephouse, D. L. et al. (2017), S. 28 & regulatory, pragmatic, normative, cultural-cognitive \\
\hline $\begin{array}{l}\text { Aldrich, H. E. / Ruef, M. / Lippmann, S. (2020), S. 195- } \\
197\end{array}$ & cognitive, sociopolitical (moral, regulatory) \\
\hline
\end{tabular}

Insgesamt kann somit auch hinsichtlich der feineren Aufgliederung der Legitimität eine große Heterogenität der taxonomischen Vorschläge konstatiert werden, welche zudem trotz ähnlicher Terminologien wiederum im Detail begrifflichinhaltlich differieren. So resümiert auch STELZER: „Die Existenz verschiedener Formen von Legitimität steht somit außer Frage, die Anzahl und Benennung dieser bleibt jedoch unklar." ${ }^{\text {"594 }}$ Darüber hinaus erscheint konzeptionell aber auch die Begriffssystematisierung vielfach eher intuitiv bzw. weniger systematisch gestaltet und inhaltlich zeigen sich (auch innerhalb der Systematiken) einige

\footnotetext{
592 Vgl. Deephouse, D. L. et al. (2017), S. 28.

593 Vgl. Beaulieu, S. / Pasquero, J. (2002), S. 54.

${ }^{594}$ Stelzer, F. (2008), S. 8.
} 
Überlappungen, z. B. bei der kognitiven vs. evaluativen Legitimitätssystematisierung. Des Weiteren erscheint aber auch begriffssystematisch die teilweise vorgenommene Integration von allgemeinen Regulierungsaspekten in einigen Konzeptionen problematisch, berührt dies doch eher die rechtliche Sphäre. Die vielfach aufgegriffene Dreiteilung in pragmatische, moralische sowie kognitive Legitimität von SUCHMAN könnte dabei zwar möglicherweise mit den Begriffen Eigennutz, Moral und Ethos (im obigen Sinne) verknüpft werden. Allerdings zeigen sich auch hier einige kritische Aspekte. So ist zum einen auch bei SUCHMAN die Terminologie eher unsystematisch gesetzt. Zudem ist bereits die Bezeichnung als ,pragmatisch“ potentiell irreführend, da Legitimitätsevaluationen im Kern auf gesellschaftlichen Moralvorstellungen beruhen, so dass letztlich alle Legitimitätskonzepte von der Moral ausgehen müssen ${ }^{595}$, bzw. die Bezeichnung als „moralische Legitimität“ wiederum redundant wäre. Der Begriffsinhalt der kognitiven Legitimität ist zwar konzeptionell interessant, da er auf verinnerlichte Werte und damit auf das Ethos bezogen werden könnte. Allerdings ist auch dieser Begriff zum einen (zumindest aus kognitionspsychologischer Perspektive) wiederum irreführend, da gerade keine Reflexion von Werten vorgenommen wird, zum anderen sind für die weiteren Ausführungen vor allem übergreifende bzw. aggregierte Werte von Bedeutung ${ }^{596}$, weshalb auch hier die Moral als Grundbegriff im Fokus steht. Auch die partikular-individualistische Begründung von Legitimität im Sinne der ,pragmatischen“ Legitimität erscheint problematisch, insofern diese nicht wiederum doch auf gesellschaftlicher Ebene legitimiert, d. h. moralisch, ist. Ähnlich stellen auch O'DWYER, OWEN und UNERMAN fest: „Attaining pragmatic legitimacy is the first step in ensuring the long term prosperity of a practice. However, a failure to attain moral legitimacy can potentially undermine the preservation of any secured pragmatic legitimacy. “597 Darüber hinaus wird das dreistufige Konzept SUCHMANS auch von PALAZZO und SCHERER kritisch evaluiert. So würden früher unkritisch akzeptierte Hintergrundannahmen (wie die Shareholder Value-Doktrin) immer häufiger hinterfragt, während

\footnotetext{
595 So stellen auch SUDDABY, BITEKTINE und HAACK fest: „The core constructs upon which the concept of legitimacy rests are norms, values, beliefs, and morals." (Suddaby, R. / Bitektine, A. / Haack, P. (2017), S. 458).

${ }^{596}$ Die Relevanz der aggregativen Legitimitätsbetrachtung heben auch BITEKTINE und HAACK hervor: ,Since it reflects the degree of collective approval of an organization [...] legitimacy is often regarded as an objective organizational resource or attribute independent of the endorsement of single individuals.“ (Bitektine, A. / Haack, P. (2015), S. 50). Ähnlich subsumiert auch BARRON: „Organizations have moral legitimacy in so far as they have the moral approval of most members of society.“ (Barron, D. N. (1998), S. 207).

597 O’Dwyer, B. / Owen, D. / Unerman, J. (2011), S. 36.
} 
eine rein individuell wie kollektiv vorteilsbegründete (,pragmatische“) Legitimität auf zunehmend gesellschaftlichen Widerstand stoße, woraus die Autoren auch die steigende Bedeutung der „moralischen“ Legitimität als unternehmerische Legitimierungsbasis herausarbeiten. ${ }^{598}$

Überdies werden jedoch auch in den vorigen Systematisierungsansätzen vielfach die zugrunde liegenden Werte und das Bewusstsein dieser Werte (,Wertewissen") kategorial vermischt. Will man nach dem Inhalt des Legitimitätsbegriffs nochmals näher und systematischer differenzieren, so erscheint eher ein Ansatz sinnvoll, welcher Legitimität in zwei Dimensionen differenziert: erstens nach dem Werteinhalt und zweitens nach der Bewusstheit der Repräsentation. Mithilfe eines solchen Ansatzes könnten die bisherigen Differenzierungen integriert werden. So steht z. B. außer Frage, dass der Moralinhalt nicht zwingend nur auf Pflichten gegenüber anderen rekurriert, wie die obigen Systematiken oftmals unterstellen, sondern durchaus auch z. B. berechtigte, d. h. gesellschaftlich legitime Eigeninteressen, -anliegen etc. enthalten (z. B. ein fair bepreistes Produkt mit guter Qualität zu erhalten), wodurch die zuvor als „moralisch/normativ“ und ,pragmatisch“ bezeichnete Legitimitätskonzeption integriert wäre. Im zweiten Schritt kann nun differenziert werden, wie konkret diese Ansprüche mental repräsentiert sind. Dabei wurde schon zuvor herausgearbeitet, dass Ansprüche nicht nur konkrete Forderungen beinhalten, sondern auch z. B. abstrakte, weniger konkretisierte und oftmals weniger bewusste Interessen. Hiermit lässt sich auch der intensiv geführte Diskurs hinsichtlich der „kognitiven Legitimität“ integrieren. Zudem gilt, dass Bewertungen sowohl von internen als auch externen Stakeholdern durchgeführt werden können ${ }^{599}$, wobei es insgesamt stets auf den aggregierten Effekt auf gesellschaftlicher Ebene ankommt, weshalb hier nochmals die Verknüpfung zum Begriff der Moral hervorgehoben sei. ${ }^{600}$ In diesem Sinne stellt auch STELZER fest, ,dass es sich bei Legitimität um eine generalisierte

${ }^{598}$ Vgl. Palazzo, G. / Scherer, A. G. (2006), S. 78. Die Autoren stellen in diesem Sinne fest: „Actors from the economic systems are immediately confronted with moral legitimacy claims, whereas during stable industrial society these claims are, as described, more or less hidden in implicit consensus on traditional customs and values (cognitive legitimacy) or the more or less accepted capitalist rhetoric of corporate economic activities contributing to the public good (pragmatic legitimacy).“ (Palazzo, G. / Scherer, A. G. (2006), S. 81).

599 So konstatieren auch RUEF und SCOTT: „Organizations relate to numerous stakeholders and confront many constituencies, both internal and external." (Ruef, M. / Scott, W. R. (1998), S. 880).

${ }^{600}$ Diese Überlegungen sind dann nochmals für das nächste Kapitel hinsichtlich des Evaluationsprozesses der Legitimität eines Unternehmens bedeutsam. Vgl. hierzu die Ausführungen in Abschnitt 4.4.3. 
Wahrnehmung handelt.“601 Die Differenzierung zwischen technischer und managementbezogener Legitimität kann wiederum herangezogen werden, um im Kontext dieser Arbeit nochmals herauszustellen, dass nicht nur das soziale System, sondern das gesamte sozio-technische System „Unternehmen“ einer Legitimitätsbewertung unterzogen wird. Weitere Systematisierungen, wie z. B. hinsichtlich einer legal fundierten Legitimität, sind schließlich eher unpassend, da diese keine saubere Abgrenzung zur rechtlichen Dimension vornehmen oder einen anderen, moralisch/rechtlich undifferenzierten Legitimitätsbegriff voraussetzen würden.

Eine weitere in der Literatur diskutierte Fragestellung befasst sich darüber hinaus mit der Konzeption des Ausmaßes an Legitimität und im Spezifischen, inwiefern Legitimität nur im binären oder vielmehr im gradualistischen Sinne gedacht werden sollte. So fassen z. B. auch in einem früheren Beitrag DEEPHOUSE und SUCHMAN Legitimität noch klar als dichotome Variable auf. ${ }^{602}$ In diesem Sinne gilt: ,,[A]n organization is either legitimate or illegitimate.“603 Diese Auffassung haben jedoch beide Autoren in einem späteren Beitrag wieder zugunsten einer ordinalen bzw. viergestuften Legitimitätsauffassung aufgegeben. ${ }^{604}$ Eine Vier- bzw. Mehrfachstufung, wenngleich mit anderen konzeptionellen Überlegungen, nehmen auch BOUTILIER und THOMSON vor ${ }^{605}$, wobei im Rahmen dieser Arbeit ein auf diesen Überlegungen aufbauender, aber explizit gradueller Ansatz gewählt sei. So erscheint es klar, dass Legitimität stets in einem gradualistischen Sinne mehr oder minder vorhanden ist, unbeschadet der Feststellung, dass diese graduelle Ausprägung nochmals konzeptionell in Subklassen unterteilt werden kann. ${ }^{606}$ In diesem Zusammenhang kann mit BOXENBAUM konstatiert werden: „An [organization] is not simply legitimate or illegitimate; it can be slightly legitimate, somewhat legitimate, or highly legitimate.“607 Es

${ }^{601}$ Stelzer, F. (2008), S. 7.

602 Vgl. Deephouse, D. L. / Suchman, M. C. (2008), S. 60. Vgl. auch Suddaby, R. / Bitektine, A. / Haack, P. (2017), S. 469.

${ }^{603}$ Deephouse, D. L. et al. (2017), S. 33. In diesem Sinne stellt auch APPLBAUM fest: „Normative power is binary, legitimacy is a normative power, so legitimacy is binary." (Applbaum, A. I. (2019), S. 147). Ebenso konstatiert auch KEMPF, dass „Legitimität keine kontinuierliche, sondern eine binäre Variable" (Kempf, M. (2007), S. 79) darstelle.

${ }^{604}$ Vgl. Deephouse, D. L. et al. (2017), S. 33.

605 Vgl. Boutilier, R. G. / Thomson, I. (2011), S. 1-3; Thomson, I. / Boutilier, R. G. (2011), S. 1784-1786.

606 Vgl. hierzu grundlegend Lingnau, V. / Fuchs, F. (2019), S. 246-247.

${ }^{607}$ Boxenbaum, E. (2008), S. 237, im Original „idea“. 
gilt folglich: ,[L]egitimacy [is] a continuous construct [...].“608 Ebenso resümiert HASSOUN: „Legitimacy [...] comes in degrees."609 Auch REINECKE, VAN BOMMEL und SPICER definieren demgemäß ,legitimacy in continuous rather that in binary terms. “" ${ }^{10}$ Intendiert man im Kontext der Legitimität eine binäre Klassifikation, so scheint eine solche Größe vielmehr die soziale „licence to operate“ $\mathrm{zu}$ repräsentieren, welche entweder vorhanden oder durch die Gesellschaft entzogen worden sein kann. ${ }^{611}$

Damit sei herausgestellt, dass für die weiteren Ausführungen nicht davon ausgegangen wird, dass Legitimität lediglich binär als vorhanden oder nicht vorhanden aufgefasst werden kann. Folglich herrscht stets ein mehr oder minder großes Maß an gesellschaftlicher Legitimität des Unternehmens vor, wobei für das normative Management der existenzgefährdende Bereich, in welchem der Entzug der sozialen , ,licence to operate ${ }^{\text {“612 }}$ droht $^{613}$, in jedem Falle zu vermeiden ist. Dabei kann jedoch auch ein größeres Maß an Legitimität sinnvoll sein,

\footnotetext{
${ }^{608}$ Boxenbaum, E. (2008), S. 237.

${ }^{609}$ Hassoun, N. (2012), S. 47, in der Quelle ebenfalls herv.
}

${ }^{610}$ Reinecke, J. / Bommel, K. v. / Spicer, A. (2017), S. 56. In diesem Sinne betont auch HELLMANN: ,Abwegig ist es jedenfalls, von einer rein binären Entscheidungssituation legitim/illegitim [...] auszugehen." (Hellmann, K.-U. (2006), S. 87). Vgl. auch Rindova, V. P. / Pollock, T. G. / Hayward, M. L. A. (2006), S. 55.

${ }^{611}$ So stellt auch HASSOUN fest: „Those who hold a binary theory of legitimacy can specify an institution is legitimate in the binary sense if it surpasses a threshold of legitimacy in the degree sense. Understanding legitimacy as a degree term allows one to specify different thresholds on legitimacy for different purposes." (Hassoun, N. (2012), S. 47, in der Quelle ebenfalls herv.). Vgl. auch Demuijnck, G. / Fasterling, B. (2016), S. 676.

${ }^{612}$ Die Begriffe der gesellschaftlichen bzw. social licence to operate und der Legitimität sind eng miteinander verwandt. Vgl. hierzu Gehman, J. / Lefsrud, L. M. / Fast, S. (2017), S. 303-305. Die Bezeichnung ist dabei der rechtlichen Sphäre einer ,legal“ licence to operate entlehnt, bezieht sich jedoch auf die gesellschaftlichen Legitimitätsvorstellungen (Moral) im Gegensatz zu rechtlich kodifizierten Normen. Sie stammt ursprünglich aus dem Kontext des Bergbaus, welcher einen besonders gesellschaftlich kritisch evaluierten und legitimierungsbedürftigen Wirtschaftssektor repräsentiert. Zum Konzept der „social licence“ vgl. Demuijnck, G. / Fasterling, B. (2016), S. 675-676; Morrison, J. (2014), S. 14-15; Prno, J. / Slocombe, D. S. (2012), S. 346-347; Thomson, I. / Boutilier, R. G. (2011), S. 1779-1786; Wilburn, K. M. / Wilburn, R. (2011), S. 3-5; Reinhardt, F. L. (2005), S. 165.

${ }^{613}$ In diesem Sinne stellt auch DEEGAN fest: ,Specifically it is considered that an organisation's survival will be threatened if society perceives that the organization has breached its social contract. Where society is not satisfied that the organisation is operating in an acceptable, or legitimate, manner, then society will effectively revoke the organisation's ,contract to continue its operations. This might be evidenced through, for example, consumers reducing or eliminating the demand for the products of the business, factor suppliers eliminating 
da dieses dann als Puffer fungiert, welcher das Unternehmen im Falle von Fehlentscheidungen in seiner Existenz schützen kann. In diesem Sinne gilt folglich: ,[L]egitimacy insulates the organization from external pressures [...].“614

Das normative Management ist folglich damit beauftragt, dafür zu sorgen, dass stets ein genügendes $\mathrm{Maß}$ an gesellschaftlicher Legitimität vorhanden ist und hat Maßnahmen zu ergreifen, um dieses aufzubauen bzw. zu erhalten. ${ }^{615}$ Die Bedeutung eines stets ausreichenden Maßes an Legitimität als zentrale Vorbedingung einer dauerhaften Existenzsicherung sowie des langfristigen Erfolgs eines Unternehmens wird vielfach in der Literatur hervorgehoben. In diesem Sinne sei nochmals betont, dass die Tätigkeit des Aufbaus und Erhalts von Legitimität gerade nicht rein appellativ bzw. als idealistischer Gedanke aufzufassen, sondern von existentieller Relevanz für den dauerhaften Unternehmenserfolg ist. So stellen auch DEMUIJNCK und FASTERLING fest: „It has been underlined by many authors that [...] organizational $^{616}$ legitimacy, is not only important as a moral ideal, but also for the survival of corporations. This implies that it can be worthwhile for an organization to take care of its legitimacy - there may be an interest for an organization to enhance its legitimacy. “617 Ähnlich resümieren auch JOHNSON und HOLUB: „Legitimacy theory is based on the idea that in order to continue operating successfully, organizations must act in a way that is socially acceptable to society. “618 Ebenso hebt auch

the supply of labour and financial capital to the business, or constituents lobbying government for increased taxes, fines or laws to prohibit those actions which do not conform with the expectations of the community." (Deegan, C. (2002), S. 293).

${ }^{614}$ Deephouse, D. L. et al. (2017), S. 30.

615 Vgl. Lingnau, V. / Fuchs, F. (2019), S. 243-247. Manche Autoren, wie z. B. Suchman, unterscheiden darüber hinaus drittens noch die Reparatur von Legitimität, z. B. nach Skandalen. Vgl. Suchman, M. C. (1995), S. 597-599 sowie die empirischen Ergebnisse bei Kuruppu, S.C. / Milne, M. J. / Tilt, C. A. (2019), S. 2070-2078; Johnson, J. / Holub, M. J. (2003), S. 283290. Die Reparatur von Legitimität sei jedoch unter den beiden ersten Begriffen subsumiert. So ist nach einem Verlust der Legitimität der Aufbau der Legitimität geboten, mit dem Ziel, mindestens das vorherige Niveau wieder zu erreichen, welches zu halten war. Vgl. hierzu auch die Überlegungen, welche in der Literatur unter dem Stichwort ,repairing trust“ intensiv diskutiert werden, so z. B. bei Bachmann, R. / Gillespie, N. / Priem, R. (2015); Eberl, P. / Geiger, D. / Aßländer, M. S. (2015); Kramer, R. M. / Lewicki, R. J. (2010); Poppo, L. / Schepker, D. J. (2010); Gillespie, N. / Dietz, G. (2009); Ren, H. / Gray, B. (2009); Schweitzer, M. E. / Hershey, J. C. / Bradlow, E. T. (2006); Child, J. / Rodrigues, S. B. (2004); Kim, P. H. et al. (2004).

${ }^{616}$ Es sei an dieser Stelle darauf hingewiesen, dass sich die Legitimitätsbewertung durch die Stakeholder im Sinne dieser Arbeit jedoch nicht auf die Organisation als unternehmerisches Teilsystem beschränkt, sondern sich auf das Unternehmen als Ganzes bezieht. Vgl. hierzu auch die Ausführungen im nachfolgenden Abschnitt 4.4.3.

${ }^{617}$ Demuijnck, G. / Fasterling, B. (2016), S. 679-680, in der Quelle ebenfalls herv.

618 Johnson, J. / Holub, M. J. (2003), S. 270. 
ZYGLIDOPOULOS hervor: „It is important for business organizations to acquire and maintain organizational legitimacy because [...] this increases the firm's chances of acquiring from its environment the resources that it needs to survive. “619 Analog stellen auch ZIMMERMAN und ZEITZ fest: „Survival is the most frequently recognized effect of legitimacy. “620 Schließlich betonen auch PALAZZO und SCHERER die Notwendigkeit ,that business firms today must reconsider their policies to maintain and reproduce legitimacy. “621 Diese zentralen Überlegungen visualisiert nochmals die nachfolgende Abbildung 4.17.

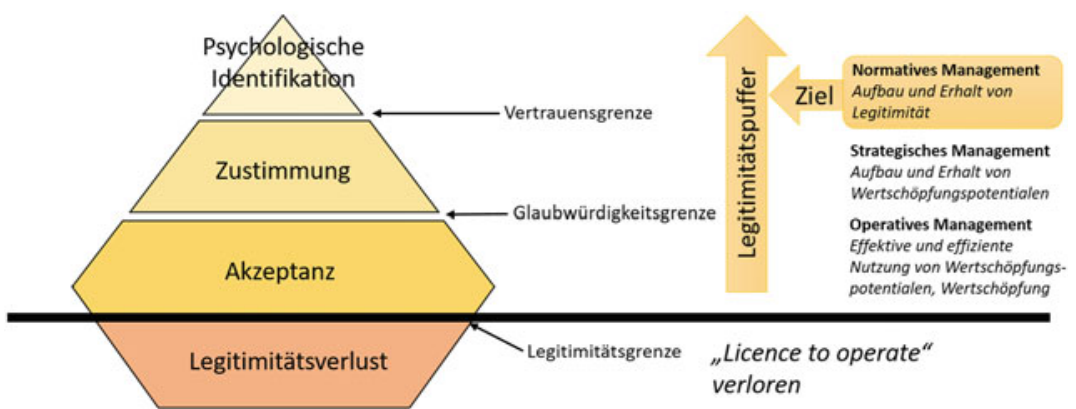

Abbildung 4.17 Normatives Management als gradualistisch orientiertes Legitimitätsmanagement $^{622}$

619 Zyglidopoulos, S. C. (2003), S. 74.

${ }^{620}$ Zimmerman, M. A. / Zeitz, G. J. (2002), S. 417. Vgl. auch Human, S. E. / Provan, K. G. (2000), S. 363; Kostova, T. / Zaheer, S. (1999), S. 64.

${ }^{621}$ Palazzo, G. / Scherer, A. G. (2006), S. 73. In diesem Sinne gilt dann auch nach MEYER und RowAN: ,The organization becomes, in a word, legitimate, and it uses its legitimacy to strengthen its support and secure its survival.“ (Meyer, J. W. / Rowan, B. (1977), S. 349). Auch PFEFFER und SALANCIK betonen: „An important part of management of the organization's environment is the management of social legitimacy. "(Pfeffer, J. / Salancik, G. R. (2003), S. 196). Schließlich konstatiert auch BouldING: „Organizations which do not create the legitimacy necessary to support them tend to disappear [...].“ (Boulding, K. E. (1974), S. 512).

622 In Anlehnung an Lingnau, V. / Fuchs, F. (2019), S. 246. Die Bedeutung von Vertrauen in die Redlichkeit des Unternehmertums kann auch mit einem klassischen, ursprünglich politikphilosophischen, Zitat von ALBERT SCHWEITZER herausgestellt werden, welcher konstatiert: ,Vertrauen ist für alle Unternehmungen das große Betriebskapital, ohne welches kein nützliches Werk auskommen kann. Es schafft auf allen Gebieten die Bedingungen gedeihlichen Geschehens.“" (Schweitzer, A. (2013), S. 128). 
Hinsichtlich der Tätigkeit des normativen Managements ergibt sich abschlieBend noch eine kurze Diskussion der Begriffe Legitimität, Legitimation und Legitimierung. Während für den Begriff der Legitimität ein Gebrauch im Sinne einer legitimierten Einheit, d. h. eines Zustandes, weitgehend unstrittig ist sowie mit dem Begriff der Legitimierung zumeist der Prozess bezeichnet wird, der diesen Zustand aufbaut bzw. erhält ${ }^{623}$, gilt dies interessanterweise gerade für den verwandten Begriff der Legitimation nicht. So verwenden manche Autoren, wie z. B. Bleicher und ABEGGLEN den Begriff im statusbezogenen Sinne, welche u. a. herausstellen, dass ,die Unternehmung [.] im Zeitablauf ihre gesellschaftliche Legitimation“624 verlieren könne. Die überwiegende Mehrheit gebraucht den Begriff jedoch prozessual ${ }^{625}$, also synonym zum Begriff der Legitimierung. ${ }^{626}$ So betont auch BITEKTINE: „Legitimacy as a property conferred on an organization by its audiences should be distinguished from legitimation, which emphasizes the process of social construction of legitimacy."627 Ebenso resümiert auch MAURER: ,Legitimation is the process whereby an organization justifies to a peer or superordinate system its right to exist, that is, to continue to import, transform and export energy, material, or information. “628 Dieser Auffassung soll

${ }^{623}$ In diesem Sinne stellt auch KoCH fest: „Der Terminus der ,Legitimität“ kennzeichnet gemäß der hier zugrunde gelegten Denotation das Ergebnis eines Prozesses der Legitimierung." (Koch, S. (2018), S. 194, in der Quelle ebenfalls herv.). Ebenso konstatiert auch RICHARDSON: „Legitimation is defined as those social processes by which legitimacy is established or defended.“ (Richardson, A. J. (1985), S. 140).

${ }^{624}$ Bleicher, K. / Abegglen, C. (2017), S. 282. Vgl. auch z. B. Núñez Nickel, M. / Moyano Fuentes, J. (2004), S. 43; Ruef, M. / Scott, W. R. (1998), S. 877; Berger, P. L. / Luckmann, T. (1967), S. 93.

${ }^{625}$ Vgl. z. B. Suddaby, R. / Bitektine, A. / Haack, P. (2017), S. 451 und S. 460; Diehl, P. (2015), S. 284; Moll, J. / Hoque, Z. (2011), S. 89; Beaulieu, S. / Pasquero, J. (2002), S. 54; Kostova, T. / Zaheer, S. (1999), S. 64-65 und S. 68; Barron, D. N. (1998), S. 213; Preston, A. M. et al. (1995), S. 510; Aldrich, H. E. / Fiol, C. M. (1994), S. 648; Rao, H. (1994), S. 29; Richardson, A. J. (1985), S. 140; Dowling, J. / Pfeffer, J. (1975), S. 126; Luhmann, N. (1975), S. 30 .

${ }^{626}$ Bei KoCH findet sich darüber hinaus auch eine weitere Systematisierung, welcher noch zwischen Legitimierung als Prozess und Legitimation als den Inhalten der Legitimierung differenziert. Vgl. hierzu Koch, S. (2018), S. 194; Koch, S. (2009), S. 127.

${ }^{627}$ Bitektine, A. (2011), S. 152, in der Quelle ebenfalls herv. Vgl. auch Wiesner, C. / Harfst, P. (2019), S. 374; Glaser, K. (2013), S. 26; Dacin, M. T. / Oliver, C. / Roy, J. P. (2007), S. 170; Dowling, J. / Pfeffer, J. (1975), S. 125. Auch SANDHU stellt in diesem Sinne fest: „Legitimität als Zustand ist das Ergebnis eines Zuschreibungsprozesses, der als Legitimation bezeichnet wird." (Sandhu, S. (2012), S. 15, in der Quelle ebenfalls herv.).

${ }^{628}$ Maurer, J. G. (1971), S. 361. 
auch im Rahmen dieser Arbeit gefolgt werden, wobei der Begriff der Legitimierung bevorzugt wird. In jedem Falle verdeutlichen die vorigen Überlegungen nochmals die Relevanz, dass stets begrifflich klar gekennzeichnet werden sollte, inwiefern der Prozess einer Legitimierung oder der normativ erwünschte Zustand der Legitimität gemeint ist.

Die vorausgegangenen Ausführungen können damit wie folgt zusammengefasst werden: Das normative Management hat aus ethischer Perspektive ${ }^{629}$ die Aufgabe, durch Legitimierung die Legitimität des Unternehmens aufzubauen bzw. zu erhalten, wobei im Kern die systematische Reflexion der an das Unternehmen gerichteten Ansprüche steht, sowie die Möglichkeit der jederzeit gesellschaftlich überzeugenden Begründung, ob und wie diese im unternehmerischen Zielsystem und konkreten Handlungen Berücksichtigung finden. Dabei handelt es sich bei der Legitimierung ersichtlicherweise nicht um einen einmaligen, sondern vielmehr einen kontinuierlichen Prozess ${ }^{630}$, woraus die Notwendigkeit eines kontinuierlichen Legitimitätsmanagements folgt. So kann auch mit EDELSTAM konstatiert werden: „Legitimacy can never be taken for granted or conferred once and for all."631 Aus diesem Grunde befasst sich das nachfolgende Kapitel detaillierter mit dem Prozess der Legitimierung, wobei nochmals zwischen der organisationalen Motivation dieser Legitimierung und dem evaluierten sozio-technischen Gesamtsystem differenziert wird.

\subsubsection{Prozess der Legitimierung}

\subsubsection{Relevanz aus neo-institutionalistischer Perspektive}

Die Relevanz einer kontinuierlichen Legitimierung kann aus konzeptioneller Perspektive mit einer bestimmten Forschungstradition des sogenannten Neoinstitutionalismus verknüpft werden. So stellen DEEPHOUSE ET AL. fest: „Legitimacy is a fundamental concept of organizational institutionalism. It influences how organizations behave and has been shown to affect their performance and survival.“632 Auch $\mathrm{KoCH}$ resümiert in diesem Sinne: „Dem Neo-Institutionalismus folgend können Organisationen ihr Überleben dann sichern, wenn sie sich auf

\footnotetext{
${ }^{629}$ Hiermit sei nochmals betont, dass normatives Management im rechtlichen Sinne bzw. als „Compliance-Management“ im Folgenden ausgeklammert und auf das normative Management im unternehmensethischen Sinne fokussiert sei.

${ }^{630}$ Vgl. hierzu auch Abschnitt 4.5.3.

${ }^{631}$ Edelstam, G. (2019), S. 145.

632 Deephouse, D. L. et al. (2017), S. 27.
} 
ihre Umwelt einstellen, um durch diese anerkannt und legitimiert zu werden. “6633 Ebenso konstatiert MIEBACH prägnant: „Die institutionellen Strukturen sichern das Überleben der Organisation [...].“634

Der Begriff des Neoinstitutionalismus bezeichnet dabei prinzipiell ein breites und relativ heterogenes Feld, wie in der Literatur häufig hervorgehoben wird. So stellen auch SCHERM und PIETSCH fest: „Der Neoinstitutionalismus bildet keine in sich geschlossene Theorie, sondern stellt eine Anhäufung sehr unterschiedlicher Sichtweisen dar [...], die eng mit den verschiedenen Perspektiven wissenschaftlicher Disziplinen verknüpft sind.“635 Aus diesem Grunde sei hier nur kurz auf den soziologischen Neoinstitutionalismus eingegangen, welcher heute ,zu den bedeutendsten Ansätzen in den Sozialwissenschaften“636 gehört. Der soziologische Neoinstitutionalismus greift hierbei im Kern die bereits existierenden grundlegenden institutionentheoretischen Überlegungen (,,alter Institutionalismus") bei WEBER, DURKHEIM, SELZNICK aber auch PARSONS wieder auf und macht diese für die moderne organisationstheoretische Forschung fruchtbar. ${ }^{637}$ Grundgedanke dieser insbesondere seit Ende der 1970er Jahren von Autoren wie Meyer und Rowan ${ }^{638}$, ZuCKer ${ }^{639}$ sowie DiMaggio und Powell ${ }^{640}$ entwickelten konzeptionellen Überlegungen ist, dass Organisationen immer in bereits wertpräformierten Umwelten existieren, an welche sie sich im normativen Sinne

${ }^{633}$ Koch, M. (2008), S. 122.

${ }^{634}$ Miebach, B. (2014), S. 84; Miebach, B. (2012), S. 133.

635 Scherm, E. / Pietsch, G. (2007), S. 66. In diesem Sinne stellt auch ScOTT fest: „Thus, the beginning of wisdom in approaching institutional theory is to recognize at the outset that there is not one but several variants." (Scott, W. R. (1987), S. 493). Ebenso konstatiert auch GoODIN ,that the ,new institutionalism" is not one thing but many." (Goodin, R. E. (1996), S. 2). Vgl. auch Funder, M. (2017), S. 10-11; Sandhu, S. (2012), S. 73; Merkens, H. (2011), S. 23; DiMaggio, P. J. / Powell, W. W. (2004), S. 1.

${ }^{636}$ Senge, K. (2011), S. 11. Vgl. auch Walgenbach, P. / Meyer, R. E. (2008), S. 11; Senge, K. / Hellmann, K.-U. (2006), S. 7.

${ }^{637}$ Vgl. Sandhu, S. (2012), S. 78; Senge, K. (2011), S. 12; Koch, S. / Schemmann, M. (2009), S. 23-24; Walgenbach, P. / Meyer, R. E. (2008), S. 11-12; Senge, K. / Hellmann, K.-U. (2006), S. 8-13; DiMaggio, P. J. / Powell, W. W. (2004), S. 11-19. So resümierte z. B. SELZNICK bereits Ende der 1940er Jahre: „Because organizations are social systems, goals or procedures tend to achieve an established, value-impregnated status." (Selznick, P. (1949), S. 256).

${ }^{638}$ Vgl. Meyer, J. W. / Rowan, B. (1977), S. 340-341.

${ }^{639}$ Vgl. Zucker, L. G. (1987), S. 443-449; Zucker, L. G. (1977), S. 726-731.

${ }^{640}$ Vgl. DiMaggio, P. J. / Powell, W. W. (1983), S. 147-150. 
anpassen müssen, um ihre Existenz zu sichern. ${ }^{641}$ Reine Effizienzüberlegungen der ökonomischen Konzeptionen, welche in den Jahrzehnten zuvor einen dominierenden Einfluss ausübten, werden aus dieser Perspektive als nicht mehr ausreichend erachtet, um den organisationalen Bestand auf Dauer zu sichern. Vielmehr muss hierzu auch die Legitimität der organisationalen Strukturen und Handlungen treten, welche sich an gesellschaftlichen Werten, d. h. der Moral, orientieren. ${ }^{642}$ Organisationen werden in dieser Hinsicht „stets innerhalb eines gesellschaftlichen Bezugssystems gesehen [.], welches gerade nicht ökonomistisch verengt operationalisiert wird."643 Der soziologische Neoinstitutionalismus versteht sich folglich auch als explizites „Gegenprojekt zu den institutionenökonomischen Ansätzen, d. h. konkret zum Transaktionskostenansatz und zur Agency-Theorie“ ${ }^{644}$ sowie zum klassischen Rational-Choice Paradigma. ${ }^{645}$ Eine Konsequenz der Adaptionsnotwendigkeit an die normative Systemumwelt ist dabei das vielfach postulierte Konzept der Isomorphie, welches darauf hindeutet, dass diese Entitäten im Laufe ihrer Geschichte durch die vorherrschenden normativen Erwartungshaltungen geprägt werden und sich normativ-institutionell angleichen. ${ }^{646}$ Hierauf aufbauend wird auch die Relevanz einer kontinuierlichen

${ }^{641}$ Zum Überblick über verschiedene Entwicklungsstufen des soziologischen Neoinstitutionalismus vgl. auch die Ausführungen bei Hasse, R. / Krüger, A. K. (2020), S. 10-13; Sandhu, S. (2012), S. 78-84; Koch, S. (2009), S. 127-128; Nee, V. (1998), S. 5-8 sowie die detaillierte historische Darstellung bei Scott, W. R. (2014), S. 1-54.

${ }^{642}$ So stellt auch PREISENDÖRFER fest: „Der Effizienzperspektive stellen die NeoInstitutionalisten eine Sichtweise gegenüber, die sich am Leitgedanken der Herstellung und Aufrechterhaltung von Legitimität orientiert.“ (Preisendörfer, P. (2016), S. 168). Hieran anknüpfend kann mit SANDHU festgestellt werden: „Organisationen sind nicht in erster Linie zielgerichtete und effiziente Strukturen, die Ressourcen optimal einsetzen, um arbeitsteilig ihre Ziele möglichst effektiv und effizient zu erreichen. [...] Ihr wichtigstes Ziel ist die Sicherstellung ihres Überlebens, indem sie Legitimität aufbauen." (Sandhu, S. (2012), S. 75). Auch КосH betont schließlich: „In der neo-institutionalistischen Organisationsforschung stellt Legitimität von Beginn an eine zentrale Kategorie dar.“ (Koch, S. (2009), S. 125). Vgl. auch Oechsler, W. A. / Paul, C. (2019), S. 75-76; Schreyögg, G. (2016), S. 74-75; Schreyögg, G. / Geiger, D. (2016), S. 477; Merkens, H. (2011), S. 23; Demers, C. (2007), S. 32-33; Hasse, R. / Krücken, G. (2005), S. 50-51; Nee, V. (1998), S. 1; Greenwood, R. / Hinings, C. R. (1996), S. 1025.

${ }^{643}$ Senge, K. (2011), S. 14. Vgl. auch Meyer, J. W. / Rowan, B. (1977), S. 348.

${ }^{644}$ Preisendörfer, P. (2016), S. 166.

${ }^{645}$ Vgl. Funder, M. (2017), S. 9-10; Miebach, B. (2014), S. 82; Miebach, B. (2012), S. 131; Müller, N. (2009), S. 222; Walgenbach, P. / Meyer, R. E. (2008), S. 120; Senge, K. / Hellmann, K.-U. (2006), S. 14.

${ }^{646}$ Vgl. Hasse, R. / Krüger, A. K. (2020), S. 11; Oechsler, W. A. / Paul, C. (2019), S. 7879; Boxenbaum, E. / Jonsson, S. (2017), S. 77-80; Preisendörfer, P. (2016), S. 168-169; 
Legitimierung bzw. eines steten normativen Managements deutlich, da sich aggregierte Werthaltungen, wie diese die Moral repräsentiert, im Zeitablauf durchaus ändern können. Die Organisation muss folglich, bereits aus Eigeninteresse der Legitimitätssicherung, darauf achten, dass bspw. ihre Handlungen nicht den vorherrschenden gesellschaftlichen Werten widersprechen.

An der vorgestellten Auffassung wurde allerdings auch in der Literatur zum Teil die Kritik geübt, dass diese zu sehr passivistisch wäre, insofern die Organisation zum einen lediglich als mehr oder minder unbewusster Rezipient von äußeren Normen (,Moral“) auftrete und zum anderen das instrumentelle Potential, d. h. die bewusste Nutzung von Legitimität als Ressource missachte. ${ }^{647}$ Auf diesen Zusammenhang weist so z. B. auch eine existierende Forschungsrichtung der Legitimierung im Sinne der Ressourcentheorie ${ }^{648}$ hin, welche z. T. als „strategisches“649 Konzept bezeichnet wird. ${ }^{650}$ In diesem Sinne stellt „Legitimität eine Ressource [dar], die eine Organisation hervorrufen und entsprechend

Schreyögg, G. (2016), S. 75; Schreyögg, G. / Geiger, D. (2016), S. 476-478; Matys, T. (2014), S. 75; Sandhu, S. (2012), S. 77; Hasse, R. / Krücken, G. (2009), S. 240; Koch, S. (2009), S. 118-120; Becker-Ritterspach, F. A. A. / Becker-Ritterspach, J. C. E. (2006), S. 102-111; Senge, K. / Hellmann, K.-U. (2006), S. 18; Hasse, R. / Krücken, G. (2005), S. 25; Kostova, T. / Roth, K. (2002), S. 215; Dacin, M. T. (1997), S. 47-48; Deephouse, D. L. (1996), S. 1024-1026; Selznick, P. (1996), S. 273; DiMaggio, P. J. / Powell, W. W. (1983), S. 150; Meyer, J. W. / Rowan, B. (1977), S. 346 und S. 352. Eine häufig aufgegriffene, weitere Differenzierung der Isomorphie findet sich bei DIMAGGIO und POWELL, welche in diesem Zusammenhang noch weiter in coercive, mimetic und normative isomorphism unterscheiden. Vgl. DiMaggio, P. J. / Powell, W. W. (1983), S. 150-154.

${ }^{647}$ Vgl. hierzu auch Schreyögg, G. (2016), S. 75; Müller, N. (2009), S. 225; Walgenbach, P. / Meyer, R. E. (2008), S. 123-124; Scherm, E. / Pietsch, G. (2007), S. 75; Senge, K. / Hellmann, K.-U. (2006), S. 29-30.

${ }^{648}$ Vgl. grundlegend Pfeffer, J. / Salancik, G. R. (2003); Peteraf, M. A. (1993); Barney, J. (1991); Wernerfelt, B. (1984) sowie die Ausführungen bei Knoll, L. (2012), S. 72; Sandhu, S. (2012), S. 186; Stelzer, F. (2008), S. 10.

${ }^{649}$ So z. B. auch bei Dacin, M. T. / Oliver, C. / Roy, J. P. (2007); Núñez Nickel, M. / Moyano Fuentes, J. (2004); Ingram, P. / Silverman, B. S. (2002); Zimmerman, M. A. / Zeitz, G. J. (2002); Deephouse, D. L. (1996); Rao, H. (1994); Oliver, C. (1991). Dieser Nomenklatur wird jedoch innerhalb dieser Arbeit nicht gefolgt, da Strategien der mittleren Managementebene zugewiesen werden, welche im Rahmen der Legitimitätsreflexion mit dem Aufbau und Erhalt von Wertschöpfungspotentialen befasst ist. Besser wäre es in diesem Zusammenhang folglich von einer ,,instrumentellen“ oder „,ressourcenorientierten“ Interpretation der Legitimierung zu sprechen.

${ }^{650}$ Vgl. Stelzer, F. (2008), S. 10-11; Oliver, C. (1991), S. 149-150. 
ihrer Ziele absichtlich, zielstrebig und kalkuliert einsetzen kann.“651 Aus diesem Grunde ist der Erhalt der Legitimität zentral „to attract resources, potential partners, and opportunities for market growth and sustainable competitive advantage. “652 Allerdings hat bereits SUCHMAN in seinem klassischen Beitrag darauf hingewiesen, dass beide Ansätze keinen Widerspruch darstellen, sondern vielmehr als zwei Seiten der Legitimierungsbetrachtung aufgefasst werden können. So stellt SUCHMAN fest: „To a large extent, of course, the distinction between strategic and institutional approaches is a matter of perspective, with strategic theorists adopting the viewpoint of organizational managers looking , out ${ }^{6}$, whereas institutional theorists adopt the viewpoint of society looking ,in ${ }^{`}[\ldots]^{\text {“653 }}$, weshalb es wichtig sei, „to incorporate this duality into a larger picture that highlights both the ways in which legitimacy acts like a manipulable resource and the ways in which it acts like a taken-for-granted belief system [...].“654 Damit befasst sich die erste Perspektive mit dem real stattfindenden, existenznotwendigen Adaptionsprozess von gesellschaftlichen Normen, während die zweite Perspektive die motivationale Komponente der Relevanz des Legitimitätserhalts bzw. eines systematischen Legitimitätsmanagements aus organisationalem Eigeninteresse in den Fokus rückt. Darüber hinaus erscheint jedoch im Rahmen dieser Arbeit auch relevant, darauf hinzuweisen, dass gerade große Organisationen nicht nur Rezipienten moralischer Normen sind, sondern auch in gewissen Zügen Einfluss auf die gesellschaftlichen Wertvorstellungen nehmen. Folglich stellt sich der Prozess der Legitimierung zwar im Kern als Bezugnahme auf gesellschaftliche Werte dar, wobei allerdings über die Zeit hinweg auch der gestalterische Einfluss hinsichtlich dieser Werte konzeptionell nicht unterschlagen werden sollte. ${ }^{655}$

${ }^{651}$ Stelzer, F. (2008), S. 10.

${ }^{652}$ Dacin, M. T. / Oliver, C. / Roy, J. P. (2007), S. 183. Ähnlich stellen auch DowLING und PFEFFER fest: „Since organizational survival is enhanced by legitimacy, legitimacy can be viewed as a resource which a given focal organization attempts to obtain and which, occasionally, competing organizations may attempt to deny.“ (Dowling, J. / Pfeffer, J. (1975), S. 125).

${ }^{653}$ Suchman, M. C. (1995), S. 577.

${ }^{654}$ Suchman, M. C. (1995), S. 577.

${ }^{655}$ Diese Überlegungen könnten prinzipiell wiederum mit den Ausführungen zur gegenseitigen Beeinflussung von Ethos (im Transfer auf die Mesoebene) und der gesellschaftlichen Moral in Verbindung gebracht werden, wie grundlegend in Abschnitt 3.1.1 bereits vorgestellt. Vgl. auch die Ausführungen des nachfolgenden Abschnitts 4.4.3.2 sowie Abschnitt 4.5.3 zur Integration im Analytischen Framework. 


\subsubsection{Zentrale Dimensionen der Legitimitätsevaluation und deren Implikationen}

Die bisherigen Ausführungen legten dabei durch die Fundierung des Legitimitätsprozesses auf Basis des Neoinstitutionalismus einen starken Fokus auf Organisationen bzw. soziale Systeme. Wie gezeigt wurde, sind Unternehmen sinnvollerweise allerdings vielmehr aus einem sozialen und technischen System bestehend zu konzipieren. Um den Prozess der Legitimierung nun genauer konzeptionell zu durchdringen, sei wieder an die grundlegenden Überlegungen des Abschnitts 4.3 angeknüpft. In diesem Zusammenhang wurde zuvor bereits herausgearbeitet, dass Unternehmen als sozio-technische Einheiten aus einem sozialen wie einem technischen Teilsystem bestehen, wobei das soziale System regelmäBig als institutionelle Organisation auftritt. Diese Überlegungen können nun mit dem Legitimitätsbegriff und insbesondere der prozessualen Dimension der Legitimierung in Verbindung gebracht werden. Dabei sei zuerst einmal festgestellt, dass Legitimität von den Stakeholdern (extern wie auch intern) mittels eines Evaluationsprozesses dem Gesamtsystem, d. h. dem Unternehmen zugeschrieben ${ }^{656}$ wird. Demgegenüber weist die institutionelle Organisation (bzw. verallgemeinert: das soziale Subsystem) das Interesse auf, die Legitimität des Gesamtsystems $\mathrm{zu}$ erhalten, um ebenfalls seine eigene Existenz zu sichern, woraus die intrinsische Relevanz von Unternehmensethik bereits zuvor begründet wurde. Um den Aufbau und Erhalt von Legitimität im Rahmen eines strukturierten normativen Managements zu ermöglichen, ist jedoch noch ein genaueres Verständnis der Evaluationsdimensionen notwendig. Zur feineren Durchdringung dieser Evaluation durch die Stakeholder wird nun wiederum auf die oben ausgearbeitete klassische Dreiteilung der Ethik hinsichtlich Intentionen, Handlungen und Konsequenzen zurückgegriffen. ${ }^{657}$ Diese Klassifikation kann dabei mit den Zielen, Handlungen sowie den Konsequenzen unternehmerischen Handelns in Verbindung gebracht

\footnotetext{
656 So kann auch mit JOHNSON und HOLUB im gesellschaftlichen Zusammenhang konstatiert werden: „Consequently organizational legitimacy is not a quality or characteristic determined by the organization itself, but is an attribute conferred on it by society." (Johnson, J. / Holub, M. J. (2003), S. 270). Ebenso stellen auch PFEFFER und SALANCIK fest: „Legitimacy is a conferred status and, therefore, always controlled by those outside the organization. As with existence of social norms generally, legitimacy is known more readily when it is absent than when it is present." (Pfeffer, J. / Salancik, G. R. (2003), S. 194). Vgl. auch Bitektine, A. / Haack, P. (2015), S. 50; Deegan, C. (2002), S. 292; Deephouse, D. L. (1996), S. 1025.

657 Vgl. hierzu nochmals Abschnitt 3.2.2.2. Ähnlich stellen aber auch DowLING und PFEFFER fest: „Organizational legitimacy is determined by the method of operation and output as well as by the goals or domain of activity of the organization." (Dowling, J. / Pfeffer, J. (1975), S. 126). Ebenso konstatieren auch DACIN, OLIVER und RoY: „In our view, legitimacy is a broader concept that pertains to the extent to which a firm's structures and activities
} 
werden und konstituieren zentrale Dimensionen der Legitimitätsevaluation. Vor diesem konzeptionellen Hintergrund ergeben sich für das normative Management folgende Implikationen: ${ }^{658}$

Erstens ist dafür zu sorgen, dass alle legitimen Ansprüche der Stakeholder bei der Analyse des unternehmerischen Zielsystems reflektiert und - wo dies möglich ist - integriert werden, welches hinsichtlich der Definition der Ziele als erwünschte zukünftige Zustände analog zu den Intentionen des klassischen philosophischen Subjekts ${ }^{659}$ gedacht werden kann. Zur Sicherstellung, dass legitime Ansprüche im unternehmerischen Zielsystem hinreichende Beachtung finden, ist, wo möglich, auf dialogische Prozesse zurückzugreifen. Die im Rahmen dieser Arbeit entwickelte Konzeption beruht damit, wie auch die Arbeiten von STEINMANN und LOHR, im Kern auf einem diskursethischen Fundament. Der diskursive Kern der Konzeption kann wiederum mit dem bereits erörterten Begriff der Verantwortung in Verbindung gebracht werden, welcher als Teilbegriff das „Antworten“ bzw. zumindest die Fähigkeit, substantiiert Antworten zu können, umfasst. ${ }^{660}$ So konstatiert auch STELZER: „Die aktive Gestaltung organisationaler Legitimität [...] ist als ein dialogischer Prozess zwischen einer Organisation und ihren Anspruchsgruppen zu konzeptualisieren. “661 In diesem Sinne übernimmt das soziale System des Unternehmens Verantwortung, indem es legitime Ansprüche diskursiv prüft oder zumindest sich potentiell einem solchen Diskurs jederzeit überzeugend ${ }^{662}$ stellen könnte. Denn in einigen Fällen ist ein regelmäBig real stattfindender Diskurs nicht möglich, so dass in diesem Falle pragmatisch

appear to conform with social norms, values, and expectations of the firm's economic and social environment.“ (Dacin, M. T. / Oliver, C. / Roy, J. P. (2007), S. 171).

658 Diese Überlegungen werden auch nochmals konzeptionell im Analytischen Framework aufgegriffen und integriert. Vgl. hierzu die Ausführungen in Abschnitt 4.5.

${ }^{659}$ Auf diese fokussiert klassisch die tugendethische Reflexion. Vgl. Abschnitt 3.2.2.

${ }^{660}$ So stellt auch SUDDABY fest, ,that, at its core, institutional theory is a theory of communication." (Suddaby, R. (2011), S. 188). Aus neoinstitutionalistischer Perspektive kann zudem mit DACIN, OLIVER und ROY konstatiert werden, dass ,organizational actions are driven by social justification, that is, by the desire of organizational actors to give a plausible and meaningful account of their actions." (Dacin, M. T. / Oliver, C. / Roy, J. P. (2007), S. 171). Vgl. hierzu auch die Ausführungen zum Verantwortungsbegriff in Abschnitt 4.1.

661 Stelzer, F. (2008), S. 12.

662 So stellt auch SUMMER fest: ,Allgemein ist eine Handlung dann nach ethischen Maßstäben richtig, wenn sie gerechtfertigt werden kann. Verantwortlich handeln heißt folglich so handeln, daß die Folgen dieses Handelns für die betroffenen Personen gerechtfertigt werden können." (Summer, L. (1998), S. 153). Dabei ist auch die Beachtung der einzelnen Stakeholdergruppen zentral, denn, so hebt auch RORTY hervor, ,justification is relative to an audience [...].“ (Rorty, R. (1995), S. 283). Vgl. hierzu auch die Ausführungen in Abschnitt 4.5.1. 
eine introspektive Formulierung (interner Monolog) ${ }^{663}$ unternehmerischer Ziele mit einer Antizipation bzw. Schätzung der existierenden Stakeholderansprüche stattfinden muss. Dies gilt z. B. für den Fall der Zeitknappheit bzw. bei Entscheidungsdruck, vielfach jedoch auch für Stakeholderansprüche, welche zwar latent existieren, aber nicht wirkmächtig artikuliert werden (können), so z. B. bei Individuen, deren Ansprüche (zumindest aktuell) nicht durch Vereinigungen ${ }^{664}$ vertreten werden oder diese selbst nicht wirkungsvoll zur Geltung bringen können. In diesem Sinne ist folglich nicht nur die gegenwärtig direkt oder indirekt diskursiv (oder auch konfrontativ!) aktive Stakeholderschaft zu beachten, welcher zumeist eine verstärkte Aufmerksamkeit zukommt, sondern auch „,passive" Stakeholder, die ihre Ansprüche gegenwärtig nicht oder zumindest nur indirekt artikulieren, insbesondere auch, weil diese Ansprüche von anderen Stakeholdern oder Anspruchsvertretern aufgegriffen und in den öffentlichen Diskurs eingebracht werden können. Grundaufgabe des normativen Managements ist es folglich, dafür Sorge zu tragen, dass möglichst alle legitimen Ansprüche im unternehmerischen Zielsystem Berücksichtigung finden. Dabei ist jedoch zu beachten, dass nicht davon ausgegangen werden kann, dass stets alle Stakeholderansprüche zueinander kompatibel sind, sondern i. d. R. immer auch gewisse Divergenzen bzw. ein gewisses Konfliktpotential aufweisen. Aus diesem Grunde existiert regelmäßig das Problem, dass nicht alle Ansprüche vollständig berücksichtigt werden können, auch wenn diese z. B. hinsichtlich Inhalt und Ausmaß prinzipiell gesellschaftlich wohlbegründet (d. h. legitimiert) sein mögen. In diesem Falle ist sicherzustellen, dass eine mögliche „Benachteiligung einzelner Stakeholder [..] auf Basis gesellschaftlich anerkannter Werte und Normen begründet “665 wird, um die Legitimität des Unternehmens zu sichern.

Ist das unternehmerische Zielsystem prinzipiell legitimiert, so ist zweitens zu prüfen, inwiefern die Handlungen unter Nutzung legitimer Mittel die Umsetzung der zuvor definierten Ziele fördern. In diesem Sinne steht „die Erreichung des Wertschöpfungszwecks stets unter einer dreifachen Legitimitätsprämisse. So muss zum Erhalt der Legitimität dauerhaft mit legitimen Mitteln eine legitime Wertschöpfung für alle Stakeholder mit legitimen Ansprüchen geschaffen werden. “666 Aus Sicht der Wirtschaftsethik der Schule um HomANN und insbesondere auch

\footnotetext{
${ }^{663}$ Vgl. hierzu auch Göbel, E. (2020), S. 58-61; Seiler, C. (2014), S. 42-44; Werner, M. H. (2011b), S. 29; Gottschalk-Mazouz, N. (2000), S. 16-17; Summer, L. (1998), S. 160-161. 664 Diese können nach FASSIN auch als „Stakewatcher“ bezeichnet werden. Vgl. Fassin, Y. (2009), S. 121.

665 Lingnau, V. / Fuchs, F. (2021), S. 13.

${ }^{666}$ Lingnau, V. / Fuchs, F. (2019), S. 244, in der Quelle ebenfalls herv.
} 
SUCHANEK ist hierbei darauf zu achten, dass diejenigen Handlungen, welche im Einklang mit den legitimen Zielen des Unternehmens stehen, gefördert werden, worauf auch PETER UlRICH hinweist. ${ }^{667}$ Darüber hinaus ist, wo eine formale Förderung der Unternehmensziele nicht möglich, sinnvoll oder gewünscht ist, auch darauf zu achten, dass zumindest keine Fehlanreize gesetzt werden. Komplementär hierzu erscheint auch die Verankerung in einer ethiksensitiven Unternehmenskultur als langfristige Gestaltungsmaßnahme im Sinne der kulturalistischen Unternehmensethik sinnvoll. ${ }^{668}$

Schließlich ist dann drittens darauf zu achten, dass mittels einer regelmäßigen Ergebniskontrolle überprüft wird, dass die legitimen Zielsetzungen mittels legitimer Tätigkeiten des Unternehmens auch erreicht werden. Dies dürfte insbesondere bei Handlungen, welche besondere gesellschaftliche Gefahrenpotentiale induzieren (z. B. Hochrisikotechnologien im Bereich der Chemie, Medizin oder Kerntechnik) von großer Relevanz sein. Hierbei ist es folglich besonders wichtig, die Konsequenzen der unternehmerischen Tätigkeiten gründlich nachzuverfolgen und jederzeit überzeugend erklären zu können, wo das Unternehmen im Sinne der Controllability ggf. keine oder nur geringe Einflussmöglichkeiten hatte. Für beide Schritte ist dabei wiederum ein dialogischer Prozess vorzuziehen, welcher die betroffenen Stakeholder, soweit möglich, regelmäßig einbezieht.

Hinsichtlich eines vertieften Verständnisses des Evaluationsprozesses der Legitimität kann in diesem Rahmen wiederum auf das Modell von TosT rekurriert werden. Sie unterscheidet dabei insgesamt drei Phasen der Legitimitätsevaluation: erstens eine initiale Urteilsbildung (,judgment formation“), zweitens die Urteilsanwendung (,,judgment use“) und drittens die Urteilsneubewertung (,,judgment reassessment"). In der Phase der Urteilsbildung hinsichtlich der Legitimität eines Unternehmens kann die Evaluierung sowohl passiv durch z. B. „Cues“ von Dritten, wie bspw. den Medien, übernommen, aber auch durch aktive Informationssuche entstehen. In diesem Sinne gilt folglich, dass ein ,legitimacy judgment is formed either through an evaluative or passive judgment mode." "669 Ähnlich resümieren auch GEHMAN, LEFSRUD und FAST diese Überlegungen wie folgt: „Stakeholders' legitimacy evaluation processes have been described as being an active judgment of the organization's observable properties and behaviours or a passive judgement relying on cues from others (regulators, media, or other

\footnotetext{
${ }^{667}$ Vgl. hierzu nochmals Ulrich, P. (2016a), S. 494.

$668 \mathrm{Vgl}$. hierzu auch die Ausführungen in Abschnitt 4.7.

${ }^{669}$ Tost, L. P. (2011), S. 694-695. Vgl. auch Bitektine, A. / Haack, P. (2015), S. 54.
} 
opinion shapers), with reassessment loops [...]."670 Somit ist sowohl die Eigendarstellung, aber auch das Bild, das Dritte über ein Unternehmen darlegen, von Bedeutung. Im zweiten Schritt ist ein Legitimitätsurteil über das zu bewertende Unternehmen gefunden und stabilisiert. Interessant erscheint dann konzeptionell insbesondere wieder der Schritt zwischen der zweiten und dritten Stufe, d. h. die Frage, wann eine Neubewertung der Legitimitätsevaluationen stattfindet. In diesem Zusammenhang stellt TosT drei Mechanismen (,jolts“, „,contradictions“ und ,reflexivity“) heraus. Für ersteres gilt dabei prinzipiell, dass allgemeine gesellschaftliche oder technologische Umbrüche zu einer Veränderung der Legitimitätsevaluationen führen können. Gleiches gilt auch zweitens für potentiell widerstreitende Werte in der Gesellschaft oder auch schlichtweg drittens für spontane Reflexionen der Legitimität, welche bei Stakeholdern auftreten können. ${ }^{671}$ Unbeschadet der Feststellung, dass die drei aufgezeigten Dimensionen kaum als konzeptionell unabhängig voneinander aufgefasst werden können, steht im Kern bei allen drei Konstrukten die Idee des ,,mental alarm“672, welche wiederum für die weiteren Ausführungen genutzt werden kann. Die erörterten Zusammenhänge fasst nun nochmals die nachfolgende Abbildung 4.18 zusammen. ${ }^{673}$

Dabei können die Überlegungen von TOST in dem Sinne fruchtbar gemacht werden, dass Stakeholder folglich sowohl aktiv nach Informationen über die Legitimität der unternehmerischen Ziele, Handlungen sowie Erklärungen über deren Konsequenzen suchen oder generell auf eine „Alarmierung“ durch Dritte setzen können, woran sich dann ggf. wiederum eine aktive Informationssuche anschließt. Gerade letzteres Verhalten dürfte dabei i. d. R. vorherrschend sein. Neben einigen überaus aktiven Stakeholdern, welche sich engagiert mit dem Unternehmen befassen, dürfte die meist übergroße Zahl an Stakeholdern darauf vertrauen, dass viele (wenn auch nicht alle ${ }^{674}$ ) Unternehmen prinzipiell legitimiert und relativ verantwortungsvoll geführt werden, da sonst Gegenteiliges über die Medien kommuniziert würde. Vor diesem Hintergrund sei hier auch nochmals die Relevanz

${ }^{670}$ Gehman, J. / Lefsrud, L. M. / Fast, S. (2017), S. 302.

${ }^{671}$ Vgl. Tost, L. P. (2011), S. 700-702.

672 Tost, L. P. (2011), S. 700. Dieser kann z. B. wiederum durch das Phänomen der kognitiven Dissonanz erklärt werden. Vgl. hierzu grundlegend Festinger, L. (1962) sowie den Überblick bei Hinojosa, A. S. et al. (2017); Morvan, C. / O'Connor, A. (2017); Cooper, J. (2007); Wicklund, R. A. / Brehm, J. W. (1976).

${ }^{673}$ Es sei angemerkt, dass sich Abbildung 4.18 auf die externen und internen Stakeholder bezieht. Aufgrund der besseren Lesbarkeit sei hier auf eine nochmalige Differenzierung wie auch eine Klassifikation nach verschiedenen Stakeholdergruppen verzichtet.

${ }^{674}$ Man denke hier bspw. an einige Unternehmen in Bereich der Textil- oder Bergbauindustrie und die Diskussion um die Einhaltung von Umwelt- und Sozialstandards. 


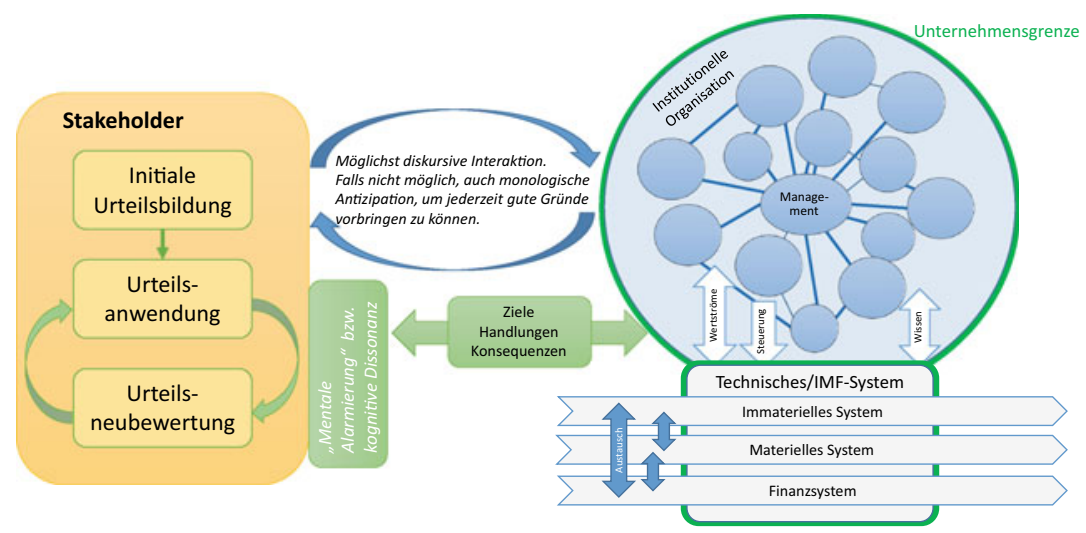

Abbildung 4.18 Modell des kontinuierlichen Legitimierungsprozesses

der Berücksichtigung legitimer Ansprüche „stiller“ Stakeholder hervorgehoben. Prinzipiell erscheint es dabei vernünftig, davon auszugehen, dass vielen Unternehmen in grundsätzlich legitimen Tätigkeitsfeldern meist im passiven Sinne eine gewisse Grundlegitimität im Sinne eines gesellschaftlichen ,goodwills“ bzw. Vertrauensvorschusses zukommt, welches im Sinne der Klassifikation von BOUTILIER und THOMSON in etwa als „Akzeptanz“ bezeichnet werden könnte. ${ }^{675}$ So stellen auch DEEPHOUSE ET AL. fest: „The overwhelming majority of organizations [...] are legitimate. Most of the time, most stakeholders passively take most organizations for granted. “676 Somit wäre in einem ersten Schritt folglich darauf zu achten, dass durch das Unternehmen keine Verletzungen der gesellschaftlich vorherrschenden Werte stattfinden, welche als Skandale in der Öffentlichkeit kritisch diskutiert werden könnten und das kognitive „Alarmsystem“ der Stakeholder aktivieren würden. Allerdings erscheint eine reine Akzeptanz noch zu gering, um die Legitimität des Unternehmens auf Dauer zu sichern. Folglich gilt auch: „Avoiding scandals is not enough!" So ist das normative Management auch diskursiv gefordert, gerade bei Großunternehmen, nicht nur passivistisch auf eine Vermeidung

675 Vgl. hierzu auch Boulding, K. E. (1974), S. 511.

${ }^{676}$ Deephouse, D. L. et al. (2017), S. 33. 
von Skandalen zu fokussieren ${ }^{677}$, sondern vielmehr auch darüber hinaus regelmäBig die Sinnhaftigkeit des unternehmerischen Tuns zu erklären, also folglich auch ein aktives Legitimitätsmanagement in der Öffentlichkeit zu betreiben ${ }^{678}$, um den Puffer an Legitimität zu erhöhen ${ }^{679}$ und das Unternehmen zu schützen. Dabei erscheint es jedoch wichtig, stets glaubhaft zu handeln und keine Maßnahmen zu ergreifen, welche durch kognitive Dissonanz bzw. Alarmierung („Argwohn“) bei den Stakeholdern gerade der Intention des Aufbaus und Erhalts der Legitimität zuwiderlaufen würde. In diesem Sinne betont auch STELZER, dass ,,vor allem ein ,zu starkes Aufbegehren“ im Rahmen der Legitimierung negative Auswirkungen haben [kann]. “680 Dies erscheint insbesondere ex post bzw. nach dem Auftreten von Skandalen bzw. gesellschaftlichen Kontroversen bedeutsam, so dass hier überzeugend das Vertrauen zurückgewonnen werden muss. ${ }^{681}$

Hinsichtlich eines aktiv-diskursiven Legitimitätsmanagements sei abschlieBend auch noch auf die vielfach diskutierte Differenz zwischen symbolischem und substanziellem Management eingegangen. So legen einige klassische Arbeiten im Rahmen des soziologischen Neoinstitutionalismus nahe ${ }^{682}$, dass es gar nicht so sehr auf substantielles Management, d. h. der genuinen bzw. ernsthaften Reflexion und Integration von legitimen Ansprüchen, ankäme, sondern vielmehr lediglich auf den „Schein“ der Legitimität gegenüber der Gesellschaft

${ }^{677}$ In diesem Zusammenhang droht dann auch die s. g. Status quo-Falle, wenn gesellschaftliche Erwartungen zunehmen, das Unternehmen aber nur wie bisher seinen Geschäften nachgeht. Vgl. grundlegend Lingnau, V. / Fuchs, F. / Beham, F. (2021).

${ }^{678}$ Vgl. hierzu auch Lingnau, V. / Fuchs, F. (2021), S. 12-14. Auch SUCHMAN unterscheidet dabei grundsätzlich ,,between seeking passive support and seeking active support.“ (Suchman, M. C. (1995), S. 574).

${ }^{679}$ So ist es, wie bereits ausgeführt wurde, auch nicht nur Aufgabe, die Legitimität zu erhalten, sondern auch diese weiter aufzubauen. Vgl. hierzu nochmals Abschnitt 4.4.2.

${ }^{680}$ Stelzer, F. (2008), S. 13.

${ }^{681}$ In diesem Sinne verweisen auch ASHFORTH und GIBBS auf die Problematik, dass Unternehmen „often ,protest too much“ their legitimacy and produce the opposite effect of that desired." (Ashforth, B. E. / Gibbs, B. W. (1990), S. 177). So können zu starke Legitimierungsmaßnahmen den Argwohn der Stakeholder hervorrufen, denn ,,[o]rganizations that are perceived by constituents as legitimate simply do not need to protest legitimacy as strongly.“ (Ashforth, B. E. / Gibbs, B. W. (1990), S. 185, in der Quelle ebenfalls herv.). JONES und PITTMAn bezeichnen diese Phänomen als s. g. ,self-promoter's paradox“ (Jones, E. E. / Pittman, T. S. (1982), S. 243).

682 So z. B. in dem vielzitierten Beitrag von MEYER und ROWAN, welche von „Rationalitätsmythen“" sprechen sowie eine Entkopplung von echten Strukturen mit behaupteten Praktiken zur Legitimierung postulieren (vgl. Meyer, J. W. / Rowan, B. (1977)). Ein solcher Ansatz wird von PREISENDÖRFER auch als „Proklamationsstrategie“ (Preisendörfer, P. (2016), S. 168) bezeichnet. Vgl. auch Hericks, K. (2020), S. 138-148. 
bzw. ggf. unwissenden Stakeholdern. ${ }^{683}$ Dies stellen auch AsHFORTH und GiBBS wie folgt fest: „There are two general means by which organizations seek legitimacy: (1) substantive management, and (2) symbolic management [...]““684 Ein solch reines normatives impression management ${ }^{685}$ zur Errichtung einer „Legitimitätsfassade“686 erscheint jedoch überaus kritisch zu evaluieren. So zeigte bspw. GRIMES im Kontext von Verschwörungsmythen bzw. -erzählungen ${ }^{687}$, dass die Geheimhaltung von Praktiken, in welchen eine Vielzahl von Individuen involviert ist, kaum auf die Dauer wahrscheinlich erscheint. ${ }^{688}$ Damit kann jedoch im Transfer auf die „wahren“ unternehmerischen Ziele und Handlungen geschlussfolgert werden, dass in großen sozialen Systemen (wie gerade im Kontext von Großunternehmen!) problematische Zielsetzungen, Handlungen, aber auch deren Konsequenzen, kaum auf Dauer verheimlicht werden können. ${ }^{689}$ Im Gegenteil ist damit zu rechnen, dass diese bereits nach kürzester Zeit publik werden, wodurch die Legitimität des Unternehmens erst recht gefährdet ist und auch hier wieder ein solches Konzept der eigentlichen Intention des Aufbaus und dem dauerhaften Erhalt der Legitimität zuwiderläuft. So stellen auch BoXENBAUM und JONSSON

${ }^{683}$ Dies resümiert auch MULLER wie folgt: „Aus dieser Perspektive muss Legitimität als eine Ressource gesehen werden, die sich Organisationen dadurch sichern, dass sie nach außen hin den Eindruck erwecken, sich an institutionalisierten Anforderungen zu orientieren." (Müller, N. (2009), S. 225).

${ }^{684}$ Ashforth, B. E. / Gibbs, B. W. (1990), S. 178. Vgl. auch Richardson, A. J. (1985), S. 141147.

685 Vgl. hierzu auch die Beiträge von Boiral, O. (2016); Lillqvist, E. / Louhiala-Salminen, L. (2014); McDonnell, M.-H. / King, B. (2013); Bansal, P. / Clelland, I. (2004); Arndt, M. / Bigelow, B. (2000) sowie den Überblick bei Bolino, M. / Long, D. / Turnley, W. (2016); Bolino, M. C. et al. (2008).

${ }^{686}$ Vgl. hierzu auch Lingnau, V. / Fuchs, F. (2021), S. 13; Rick, S. (2018), S. 11; Greiling, D. (2009), S. 386; Süß, S. (2009), S. 121-122; Scherm, E. / Pietsch, G. (2007), S. 70-71.

${ }^{687}$ In der fachwissenschaftlichen Literatur wird häufig der alltagssprachlich geläufige Begriff der Verschwörungstheorie kritisch evaluiert, da die in Verschwörungen generierten Postulate nicht den Anforderungen wissenschaftlicher Theorien genügen. Vgl. hierzu bspw. Nocun, K. / Lamberty, P. (2020), S. 21-24.

688 Vgl. Grimes, D. R. (2016), S. 3-6.

${ }^{689}$ Darüber hinaus sind auch negative Effekte auf die Motivation bzw. das Commitment der internen Stakeholder, welche zuvor bereits über die illegitimen Praktiken Bescheid wissen, zu beachten. Vgl. hierzu auch die Überlegungen zur Reparatur von internen Vertrauensverlusten bei Gillespie, N. / Dietz, G. (2009), S. 133-141; Child, J. / Rodrigues, S. B. (2004), S. 146-147. 
fest: „Institutional decoupling carries with it a risk of detection where it would no longer confer legitimacy, but probably shame, on the organization." 690

Gleiches gilt auch für einige weitere in der Literatur neben einer Adaption an gesellschaftliche Normen vorgeschlagene Handlungsalternativen. So stellt z. B. SUCHMAN neben einer reinen Konformität auch die Alternativen der Selektion bestimmter zu adressierender Subgruppen der Gesellschaft sowie die Manipulation vor. ${ }^{691}$ Beide letztgenannte Alternativen erscheinen für eine dauerhaft erfolgreiche Legitimierung jedoch weniger zielführend. Scheidet eine intendierte Manipulation bzw. Täuschung mit ähnlichen Argumenten wie beim Scheinmanagement spätestens durch die Diffusion dieser Intention an die Öffentlichkeit aus, so ist auch eine lediglich partielle gesellschaftliche Ansprache von Subgruppen kritisch, falls diese es versäumt, eine hinreichende Masse an Stakeholdern zu überzeugen. Als vierte viable Option könnte jedoch, auch der Bezeichnung nach zutreffender, eine wohlverstandene Mitbegründung neuer gesellschaftlicher Werte konzipiert werden, wie diese bei ZIMMERMAN und ZEITZ als Erweiterung der Überlegungen SuCHMANS vorgestellt wird. ${ }^{692}$ So gestalten gerade große Unternehmen evident mit ihrer Existenz auch gesellschaftliche Werte mit bzw. prägen Lebensstile und Wertvorstellungen. Allerdings ist hier wiederum zu beachten, dass zum einen eine solche normative Mitgestaltung auf akzeptierten gesellschaftlichen Normen aufbaut und klar von intendierten Manipulationsversuchen ohne Bereitschaft der eigenen Adaption bzw. kritischen Reflexion abgegrenzt ist. Hier kommt es folglich wieder auf gute Gründe ${ }^{693}$ und eine überzeugende diskursive gesellschaftliche Beteiligung an. Zum anderen ist jedoch auch zu beachten, dass gesellschaftliche Werte sich meist eher mittel- bis langfristig ändern, so dass ein solcher Ansatz auch eher auf dieser Zeitskala als wirksam für das normative Management zu erachten wäre. Die vorausgegangenen Überlegungen subsumiert nochmals die nachfolgende Abbildung 4.19.

${ }^{690}$ Boxenbaum, E. / Jonsson, S. (2017), S. 87.

${ }^{691}$ Vgl. Suchman, M. C. (1995), S. 587-593.

692 Vgl. Zimmerman, M. A. / Zeitz, G. J. (2002), S. 423. Diese zweite Möglichkeit heben so auch PFEFFER und SALANCIK wie folgt hervor: „That is, the organization may conform to social values. Alternatively, the organization may attempt to change the social definition of legitimacy with respect to its own operations and objectives." (Pfeffer, J. / Salancik, G. R. (2003), S. 196). Vgl. auch Stelzer, F. (2008), S. 12 sowie die Klassifikation bei Oliver, C. (1991), S. 152; Dowling, J. / Pfeffer, J. (1975), S. 127.

${ }^{693} \mathrm{Vgl}$. hierzu die Ausführungen im folgenden Abschnitt 4.5. 


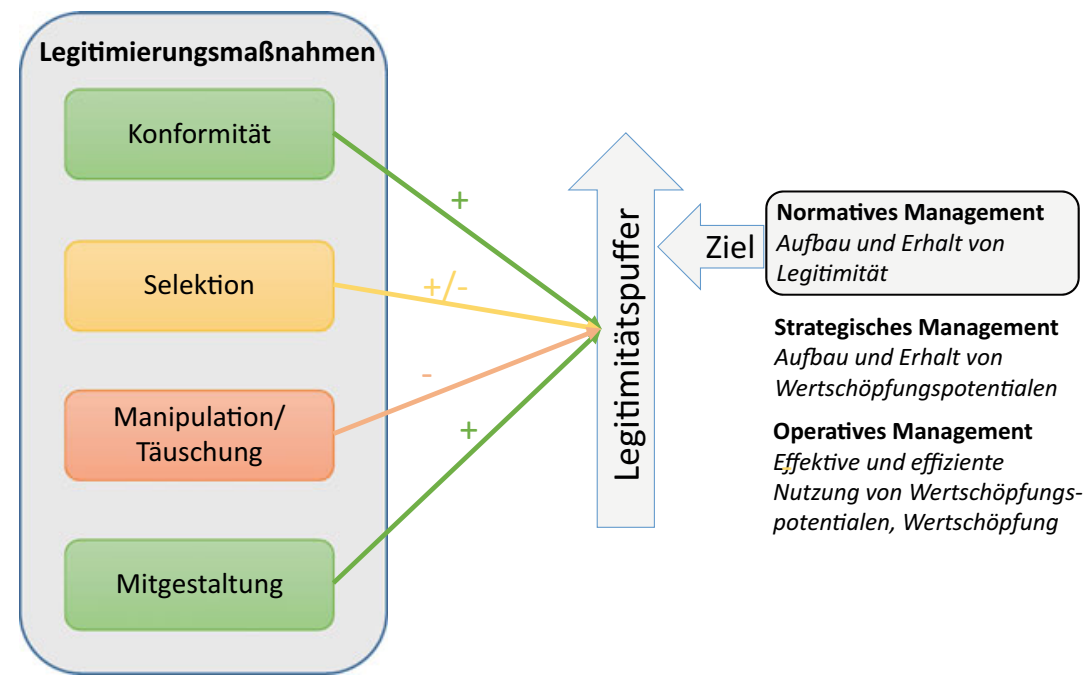

Abbildung 4.19 Langfristiger Einfluss möglicher Legitimierungsmaßnahmen

Für einen dauerhaften Legitimierungserfolg sinnvoll kann somit zusammengefasst nur die Anpassung an bzw. Berücksichtigung von Werten sowie die diskursive Auseinandersetzung als normative (Mit-)Gestaltung erachtet werden, die mit guten Gründen überzeugend im gesellschaftlichen Diskurs tätig wird. Dauerhaft erfolgreiches normatives Management ist somit in jedem Falle als substantielles Management zu implementieren. Um ein solches normatives Management systematisch betreiben zu können, bedarf es jedoch wiederum der Instrumente, die ein strukturiertes und planmäßiges Vorgehen ermöglichen. Nachdem die Arbeit nun zuvor die konzeptionellen Grundlagen der Unternehmensethikkonzeption detailliert erarbeitet hat, fokussieren die Ausführungen im Weiteren auf die praktische Nutzbarkeit der zuvor gewonnenen Erkenntnisse im Sinne der Klassifikation nach STOKES. Dazu wird, wie bereits einleitend ausgeführt, im Weiteren mit dem Analytischen Framework ein Instrument entwickelt, welches das normative Management in der Praxis hinsichtlich eines strukturierten (bzw. planmäßigen! ${ }^{694}$ ) Vorgehens im Rahmen der Funktion des Aufbaus und Erhalts der unternehmerischen Legitimität zu unterstützen vermag.

${ }^{694}$ Wie bereits aufgezeigt wurde, stellt ein hinreichend planmäßiges Vorgehen als Teilelement der Mindestprofessionalität eine konstitutive Eigenschaft von Unternehmen dar. Vgl. hierzu Abschnitt 4.3.5. 


\subsection{Das Analytische Framework als Instrument eines strukturierten Legitimitätsmanagements}

\subsubsection{Konzeptionelle Grundlagen des Frameworks}

Wie bereits zuvor dargestellt wurde, findet sich eine praktisch orientierte Unternehmensethik, welche weniger streng konzeptionell, dafür jedoch eher in praktischen Problemfeldern tätig wird, vor allem im angelsächsischen Raum, in welchem auch einige Autoren s. g. ethical frameworks entworfen haben, die eine strukturierte, ethisch begründete bzw. reflektierte normative Argumentation unterstützen. ${ }^{695} \mathrm{Im}$ deutschsprachigen Raum stellen solche Ansätze jedoch nach wie vor eher eine Ausnahme dar. Eine dieser wenigen Ausnahmen repräsentiert das analytische Framework von KÜPPER, welches im Weiteren zum Ausgangspunkt des Entwurfes eines eigenen Ansatzes genommen wird. Als Vorteil der Konzeption KÜPERS kann dabei sicherlich zum einen die Praxisnähe sowie die Möglichkeit einer systematischen Reflexion ethischer Probleme hervorgehoben werden. Wie KUPPER richtigerweise feststellt und bereits einführend dargestellt wurde, sind normative Fragen nicht letztbegründbar (s. g. Münchhausen-Trilemma ${ }^{696}$ ). In diesem Sinne scheint gerade für die BWL, mit ihrem historisch begründet, relativ kritischen Standpunkt zu ethisch-normativen Fragestellungen, ein analytischer Zugang fruchtbar. Hierbei geht es folglich nicht darum, normative Fragen für die Praxis letztklären oder appellativ argumentieren zu wollen, sondern der wissenschaftliche Anspruch besteht vielmehr darin, Instrumente zu entwickeln, mit welchen die Argumentation des normativen Managements systematisch unterstützt und damit die diskursive Transparenz und Präzision geschärft werden kann. Dabei muss im Ansatz von KÜPPER allerdings festgestellt werden, dass die von ihm fokussierten „ethischen Probleme“ noch relativ vage bleiben bzw. kaum spezifiziert werden und damit in ihrer Detektion weitestgehend der Intuition des Anwenders überlassen bleiben. ${ }^{697}$

Aus diesem Grunde soll im Rahmen dieser Arbeit ein wesentlich fundamentalerer Zugang zu einem analytischen Framework gelegt werden, welches mit der systematischen und kritischen Analyse der Ansprüche der Stakeholder beginnt, mit dem Ziel, eine strukturierte, ausgewogene und überzeugende Begründung für

\footnotetext{
695 In diesem Zusammenhang sei z. B. nochmals das Framework von FISHER und LOVELL hervorgehoben. Vgl. hierzu Fisher, C. / Lovell, A. (2009), S. 139-140 sowie die Ausführungen in Abschnitt 3.5.

696 Vgl. hierzu Abschnitt 2.5.1.

697 Vgl. Küpper, H.-U. (2011), S. 172.
} 
das normative Management zu schaffen, mit welchem dieses transparent darlegen kann, welche Ansprüche in welchem Ausmaß erfüllt werden (können), um die Legitimität des Unternehmens zu sichern. Als konzeptionelle Grundlage kann dabei auf die Stakeholder Theory zurückgegriffen werden, welche insbesondere von EDWARD FREEMAN in den wissenschaftlichen Diskurs eingebracht wurde. ${ }^{698}$ Grundgedanke dieses Ansatzes ist, dass Unternehmen einer Vielzahl von Anspruchsgruppen gegenüberstehen, welche die Unternehmensführung beachten sowie deren begründete Ansprüche substantiell wie überzeugend gegeneinander abwägen und soweit möglich integrieren muss, um das Unternehmen dauerhaft erfolgreich zu führen. Ein einseitiger Fokus auf bestimmte Stakeholdergruppen, wie z. B. die Eigenkapitalgeber, mit rein instrumenteller Betrachtung der anderen Stakeholdergruppen, wie dies z. B. die Shareholder Value-Theorie postuliert, wäre lediglich unter den Idealprämissen der traditionellen neoklassischen Theorie (vollkommene Märkte, vollständige Verträge etc.) plausibel und impliziert in realen Handlungskontexten die existenzielle Gefahr eines Legitimitätsverlustes. ${ }^{69}$ Folglich hat die Unternehmensführung im Rahmen einer normativ reflektierten Stakeholder-Theorie alle legitimen Ansprüche der internen und externen Stakeholder zu berücksichtigen und diese auszubalancieren. So konstatiert auch BEMMELS: „There is a long list of interest groups that exercise influence in the head offices and boardrooms of the world [..]. Employees and unions, customers and consumer advocates, bankers and creditors, environmentalists, governments and the public at large all have legitimate interests that you have to balance off against the shareholders' desire to make money. “700 Ebenso kann auch mit CAMPBELL und ALEXANDER festgestellt werden: „A company must give a stream of value to each stakeholder [...]. If stakeholders do not perceive such value, they will redirect their loyalty, either

${ }^{698}$ Nach FREEMAN tauchte der Begriff des Stakeholders bereits 1963 in einem internen Memorandum des Stanford Research Institutes auf. Vgl. hierzu Freeman, R. E. / Reed, D. L. (1983), S. 89. FREEMAN kann jedoch zweifelsohne als der zentrale Begründer der akademischen Stakeholderforschung gesehen werden, weshalb dieser in der Literatur auch als „father of stakeholder theory“ (Maak, T. / Liedekerke, L. v. (2007), S. 301; Walsh, J. P. (2005), S. 429; Werhane, P. H. (2003), S. 82) bezeichnet wird. Vgl. hierzu auch Malecki, C. (2018), S. 281 sowie kritisch Bowie, N. E. (2012), S. 179. Zu den Grundlagen der Stakeholder Theory vgl. auch Freeman, R. E. (2010a); Freeman, R. E. (2010b); Freeman, R. E. et al. (2010); Freeman, R. E. / Martin, K. / Parmar, B. (2007); Freeman, R. E. / Wicks, A. C. / Parmar, B. (2004); Phillips, R. / Freeman, R. E. / Wicks, A. C. (2003).

${ }^{699}$ Vgl. Lingnau, V. / Fuchs, F. (2021), S. 13-14; Lingnau, V. (2011a), S. 39.

${ }^{700}$ Bemmels, B. (2004). 
gradually or precipitously. “701 Die vorausgegangenen Überlegungen subsumiert nochmals die nachfolgende Abbildung 4.20.

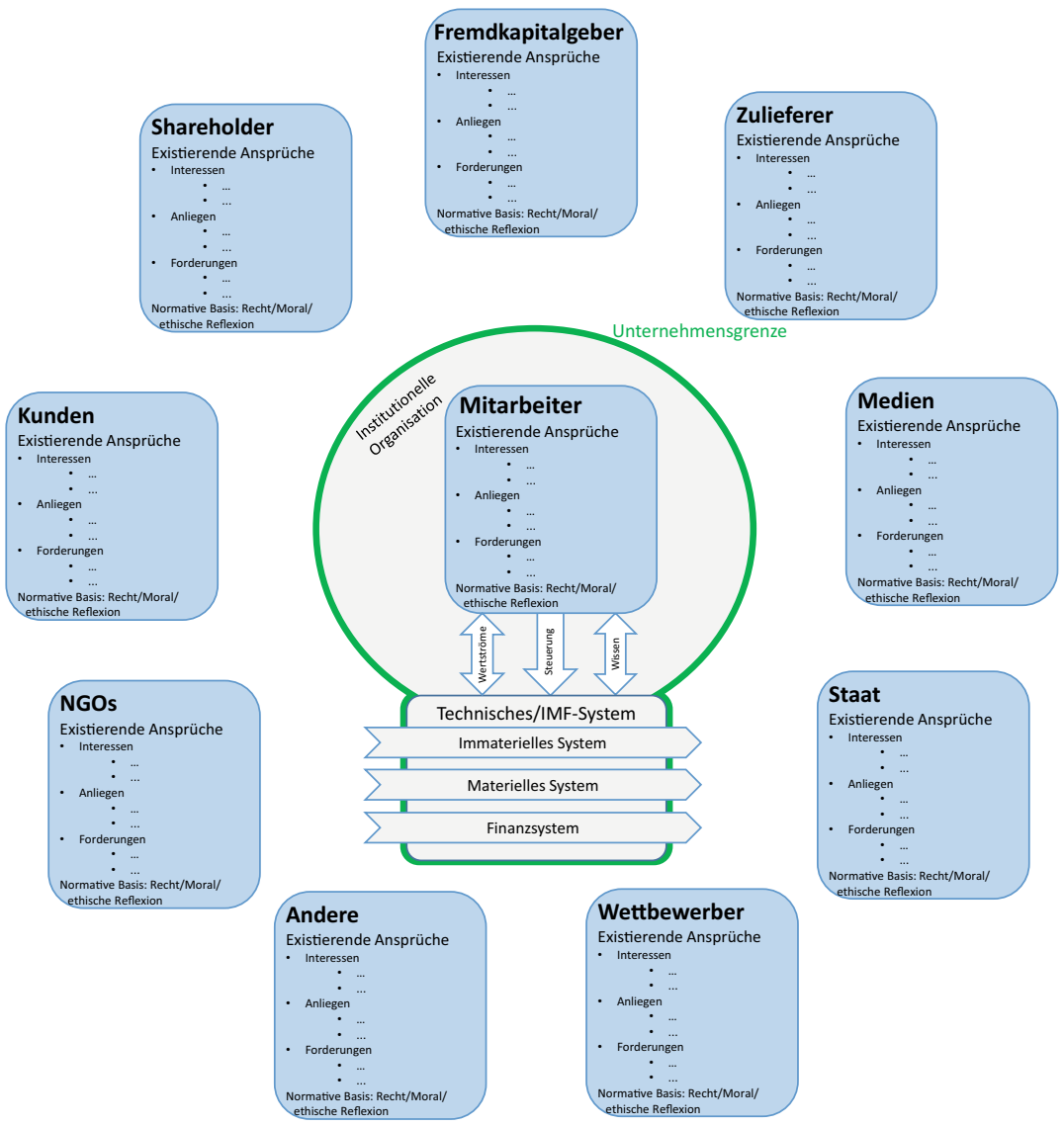

Abbildung 4.20 Überblick über die Anspruchsgruppen des Unternehmens ${ }^{702}$

${ }^{701}$ Campbell, A. / Alexander, M. (1997), S. 44.

${ }^{702} \mathrm{Vgl}$. grundlegend auch Freeman, R. E. (2010a), S. 25. Es sei angemerkt, dass auch das Management zu den internen Stakeholdern bzw. Mitarbeitern gehört. Aus Übersichtlichkeitsgründen wurde jedoch auf eine explizite Darstellung in der Abbildung verzichtet. 
Dabei ist jedoch zu klären, welcher normative Maßstab bei der Evaluation der Stakeholderansprüche zugrunde zu legen ist. Wie die obigen Ausführungen aufzeigten, kann ein lediglicher Rekurs auf Rechtsnormen (ComplianceManagement) als nicht hinreichend für ein normatives Management erachtet werden, um dauerhaft die Legitimität des Unternehmens zu sichern. Allerdings ist auch die konzeptionell bis heute dominierende rein faktisch-deskriptive Interpretation des Legitimitätsbegriffs nicht unumstritten geblieben, so dass für die Konzeption des Analytischen Frameworks auf eine zweistufige Legitimitätskonzeption zurückgegriffen wird. Die Relevanz eines solchen Konzepts kann auch nochmals mit den Überlegungen von DIEHL fundiert werden, welcher feststellt: „Aktuellere Versuche, den Begriff der Legitimität zu präzisieren, zielen auf die Trennung zwischen deskriptiver und normativer Perspektive.“703 Ein solch differenzierter Ansatz, welcher eine rein faktisch-deskriptive Legitimitätsanalyse mittels einer kritisch-deskriptiven, aber auch ggf. ethisch-normativen Reflexion weiterführt, wird im Folgenden vorgestellt. ${ }^{704}$

\subsubsection{Zweistufiges Konzept der Legitimitätsevaluation}

Die erste Stufe dieses zweistufigen Ansatzes beruht dabei auf einem empirischen Zugang zur Legitimität und fokussiert damit auf den gesellschaftlich faktisch vorherrschenden Bestand an Moral. Der in diesem Kontext geläufige Begriff einer Wertegemeinschaft kann dabei mit den bereits in den Grundlagen eingeführten Begriff des „overlapping consensus“ nach RAWLS gestützt werden, welcher davon ausgeht, dass trotz aller Differenzen ein gesellschaftlicher Mindestbestand an gemeinsamen Normen vorherrschen muss, damit eine Gesellschaft dauerhaft

\footnotetext{
703 Diehl, P. (2015), S. 283.

${ }^{704}$ So stellen auch BESCHORNER und NUTZINGER fest: „Diese [..] Kluft zwischen deskriptiver und normativer Ethik, zwischen Anwendung und Begründung, lässt sich empirisch als eine ,Lücke', wenn nicht gar als ein ,garstiger Graben' auffassen, die es zu schließen und zu überwinden gilt. Wichtig wird sein, diese Kluft dadurch zu überbrücken, dass beide Perspektiven, die Sicht der deskriptiven und die der normativen Ethik, zugleich behandelt und sie dadurch stärker aufeinander bezogen werden, als dies in der bisherigen wirtschaftsethischen Diskussion gemeinhin der Fall ist. Nur durch die simultane Abarbeitung einer deskriptiven und normativen Ethik ist es nach unserer Einschätzung möglich, eine problemadäquate Perspektive für die Wirtschafts- und Unternehmensethik zu entwickeln, die uns dringend notwendig erscheint." (Beschorner, T. / Nutzinger, H. G. (2007), S. 245-246, in der Quelle ebenfalls herv.).
} 
existieren kann. ${ }^{705}$ So stellen Gesellschaften zwar keineswegs absolut homogene Werteinheiten dar und weisen vielfältige Partikularwerte in spezifischen Subkulturen auf, dennoch ist die Akzeptanz grundlegender gemeinsamer Werte (z. B. in freiheitlichen Gesellschaften die liberale Demokratie, Rechtsstaatlichkeit), zumindest bei der großen Mehrheit der Individuen, unabdingbar. Komplexer wird eine Betrachtung noch, wenn man an global operierende Unternehmen denkt, die in einer Vielzahl von im Detail differierenden Wertekontexten tätig sind. Hierbei können sich durchaus Wertedifferenzen ergeben, welche sich z. B. in der Existenz zusätzlicher Werte, aber auch im Detail differierender Interpretationen der als richtig empfundenen Reaktion auf einen Verstoß gegen einzelne Werte (z. B. kollektiver Scham im asiatischen Kulturraum) ergeben. Aus Perspektive der Sicherung der unternehmerischen Legitimität müssen damit in der Region, in welcher Geschäfte betrieben werden, aus empirischer Sicht in einem ersten Schritt faktisch die vorherrschenden, kulturspezifischen Werte Beachtung finden, um die Legitimität des Unternehmens zu sichern. In diesem Sinne handelt es sich bei der ersten Stufe der Legitimität, welche auch zentral für das normative Management im Sinne des strukturierten Legitimitätsmanagements ist, um die notwendige Legitimität, denn diese ist notwendige Voraussetzung, um in einer Wertegemeinschaft dauerhaft erfolgreich den Geschäften nachgehen zu können.

An einer rein faktischen, empirischen Fundierung der Legitimität ist jedoch auch Kritik geübt worden, da diese zu relativistisch sei und damit (zumindest hypothetisch) auch eine „Kultur der Teufel“ unkritisch zum Maßstab der unternehmerischen Legitimierung machen könne, welches wiederum, wie aufgezeigt, neuerdings bei MELÉ und ARMENGOU zur Entwicklung eines normativen Legitimitätskonzepts führte, welches explizit auf einem ethisch-normativen Fundament aufbaut. ${ }^{706}$ Kritik an einer rein faktisch-deskriptiven Konzeption kommt im wirtschaftsethischen Zusammenhang auch von PETER ULRICH, welcher eine rein an den faktischen Normbeständen ansetzenden Argumentation einer deutlichen Kritik unterzieht und als „Reflexionsstopp“ vor lediglich scheinbaren Normtatsachen bezeichnet. ${ }^{707}$

Dieser Kritik kann nun mit einer zweiten Stufe der Legitimitätsevaluation begegnet werden. Dabei ist in einem ersten Schritt klar, dass eine dauerhafte Existenzsicherung die Berücksichtigung der faktisch vorherrschenden Moral (ungeachtet dessen, wie diese nun sein mag) schlicht notwendig ist, um in einem

705 Vgl. hierzu nochmals Rawls, J. (2005), S. 133-172; Rawls, J. (1999), S. 340; Rawls, J. (1987), S. 1 sowie die Ausführungen in Abschnitt 3.1.1.

706 Vgl. Melé, D. / Armengou, J. (2016), S. 730.

${ }^{707}$ Vgl. Ulrich, P. (2017), S. 14; Ulrich, P. (2016a), S. 15. Vgl. auch Ulrich, P. (2004b), S. 17. 
bestimmten Werteumfeld erfolgreich zu sein. Darüber hinaus bleibt es dem Management unbenommen, in einer gesellschaftlichen Umgebung, welche aus einer kritischen Perspektive heraus, bestimmten, als fundamental erachteten Werten diametral zuwiderläuft, keine Geschäfte zu betreiben. ${ }^{708}$ Dies kann dann allerdings möglicherweise kurzfristig das Ende der Geschäftstätigkeit bedeuten, oder, jedoch langfristiger gedacht, potentiell zu ergreifende Maßnahmen implizieren, um überzeugend auf eine stetige Änderung (oder auch Durchsetzung) der (ggf. auch in der Bevölkerung bereits latent) vorherrschenden Werte einzutreten und seinen unternehmerischen Einfluss dementsprechend einzusetzen. Überdies ist eine kritische Evaluation vorherrschender Moralstandards auch bei einer Evaluation bzw. Ausbalancierung sich ggf. widersprechender Werte sinnvoll, wie diese gerade im Kontext einer globalen Wirtschaftstätigkeit auftreten können. Da, wie wissenschaftstheoretisch dargelegt und einleitend nochmals hervorgehoben wurde, solche Wertekonflikte nicht letztgelöst werden können, ${ }^{709}$ ist auch hier ein Rückgriff auf bestimmte grundlegend anerkannte oder auch ethisch-normativ begründete Werte als Orientierungswissen hilfreich.

Als Basis einer solchen kritischen Evaluation kann folglich selbst wiederum zweistufig vorgegangen werden. In einem ersten Schritt kann hierbei auf einer bestimmten Forschungsrichtung der deskriptiven Ethik, allerdings mit kritischem Fokus, aufgebaut werden. Als konzeptionelle Grundlage dient zum einen die s. g. Common Morality Theory, welche bspw. prominent von ARNOLD, BEAUCHAMP, BOWIE sowie CHILDRESS vertreten wird. ${ }^{710}$ Diese postuliert, dass es auch auf der globalen Ebene gemeinsame Grundwerte gibt, welche in ihrer prinzipiellen Richtigkeit nicht ernsthaft bestritten werden können ${ }^{711}$, auch wenn sie bisher

${ }^{708}$ Darüber hinaus könnte auch der formale Betreiber des Unternehmens, d. h. z. B. die (Aktien-)Gesellschaft als juristische Person, adressiert werden, da diese grundlegend für die Aufrechterhaltung oder Einstellung der Geschäftstätigkeit verantwortlich ist. Vgl. Lingnau, V. / Fuchs, F. (2019), S. 249.

${ }^{709}$ Vgl. Abschnitt 2.5.1.

${ }^{710}$ Vgl. Arnold, D. G. / Beauchamp, T. L. / Bowie, N. E. (2020), S. 29-32; Beauchamp, T. L. / Childress, J. F. (2013), S. 2-5 und S. 410-411. So stellt auch DE GEORGE fest: „Some general ethical norms apply to any business operating anywhere. These norms are universally applicable because they are necessary either for a society to function or for business transactions to take place. They are widely held, and everyone is expected to live by them and up to them; they are obvious, common-sensical, and available to all." (De George, R. T. (1993), S. 19).

${ }^{711}$ Diese Position steht folglich dem s. g. Wertrelativismus gegenüber, welcher postuliert, dass die allgemeine „Richtigkeit“ von Werten nicht beurteilt werden kann und jede Kultur eigene, letztlich nicht zu kritisierende Werte hervorbringt. Der universalistische Standpunkt, welcher den aufgezeigten Überlegungen zugrunde liegt, geht dagegen davon aus, 
nicht immer umfassend verwirklicht sein sollten. ${ }^{712}$ Diese prinzipiell anerkannten gemeinsamen Grundwerte sind auch für eine zukünftige friedliche Koexistenz auf globaler Ebene von entscheidender Relevanz. In diesem Sinne kann mit HÖFFE konstatiert werden: „Schon die Notwendigkeit eines ebenso friedlichen wie gerechten Zusammenlebens der angedeuteten Vielfalt erfordert die Anerkennung gewisser Grundsätze. Zu ihnen gehören außer der Menschenwürde die liberalen Freiheitsrechte und elementare Grundsätze der Gerechtigkeit.“713

Die vorausgegangenen Überlegungen werden schließlich auch auf empirischer Ebene in Zusammenhang mit der s. g. Weltethosforschung in der Tradition von HANS KUNG gestützt, welche auf deskriptivem Wege gemeinsame Grundwerte wie den Schutz des menschlichen Lebens, Verzicht auf Gewalt, Gerechtigkeit ${ }^{714}$,

dass (zumindest grundlegende) wohlbegründete, bzw. ethisch fundierte Normen existieren, wodurch auch eine Evaluation aktuell praktizierter Werte von (Sub-)Kulturen möglich ist. Beide Positionen sind in der Geschichte der Ethik intensiv diskutiert worden. Vgl. hierzu konzeptionell auch Arnold, D. G. / Beauchamp, T. L. / Bowie, N. E. (2020), S. 7-11; Crane, A. et al. (2019), S. 87-88; Härle, W. (2018b), S. 16-19; Lütge, C. / Uhl, M. (2017), S. 139140; Shaw, W. H. (2017), S. 13-14; Fisher, C. / Lovell, A. (2013), S. 417-424; Homann, K. / Lütge, C. (2013), S. 103-104; De George, R. T. (2010), S. 27-33; Fischer, J. et al. (2008), S. 37-38; Hepfer, K. (2008), S. 20-21; Wieland, J. (2007), S. 112-115; Schweppenhäuser, G. (2006), S. 10; French, W. A. / Granrose, J. (1995), S. 65.

712 So stellt auch BAIN fest: „Some values can only be evaluated relative to the context, but some moral norms are accepted by all. In no culture is it permissible to kill a neighbour arbitrarily [...]. There is a widespread support for other basic norms, such as respecting human rights.“ (Bain, W. A. (1997), S. 125). Vgl. auch die Überlegungen bei Wettstein, F. / Waddock, S. (2005), S. 307-309, welche in ihrer kritischen Reflexion ebenfalls auf die allgemeinen Menschenrechte zurückgreifen. In diesem Zusammenhang ist auch die Frage zu stellen, inwieweit Grundnormen wie die allgemeinen Menschenrechte tatsächlich kulturabhängig sind, oder ob das Argument der kulturellen Differenzen nicht häufig aus politischen Motiven vorgeschoben wird. So liegt es nach BIELEFELDT nahe, dass gerade autoritäre Regime vielfach „Argumente des Kulturpluralismus politisch instrumentalisieren, um sich menschenrechtliche Kritik vom Halse zu halten. In der Debatte um das Verhältnis von Menschenrechten und kulturellem Pluralismus gilt es daher immer wieder zu fragen: Wer sind denn diejenigen, die sich in der internationalen Politik als Repräsentanten der kulturellen Identität ihrer Völker ausgeben? Wer hat sie in ihrer Sprecherrolle legitimiert? Und was ist das für eine ,Kultur', die mit Panzern gegen demonstrierende Studenten durchgesetzt werden muß?“ (Bielefeldt, H. (1999), S. 43).

${ }^{713}$ Höffe, O. (2018), S. 108-109. Ebenso konstatiert auch PAUER-STUDER: „Wichtig für eine einigermaßen stabile Koexistenz, die nicht sofort durch jede neu auftretende Kontroverse über ethische Probleme in Frage gestellt wird, ist die Herstellung eines gemeinsamen Wertekonsenses.“ (Pauer-Studer, H. (2020), S. 19).

${ }^{714}$ Das Konzept der Gerechtigkeit ist wissenschaftshistorisch intensiv bearbeitet worden. So differenziert z. B. ARISTOTELES klassisch zwischen geometrischer bzw. distributiver Gerechtigkeit und arithmetischer Gerechtigkeit bzw. Tauschgerechtigkeit (vgl. Aristoteles (2015), 
Wahrhaftigkeit ${ }^{715}$ etc. als gemeinsames Wertefundament aufzeigt. ${ }^{716}$ Darüber hinaus kann jedoch auch, gerade im akademischen Kontext, in einem zweiten Schritt noch eine kritische Reflexion der vorherrschenden Moralvorstellungen anhand ethisch-normativer Überlegungen stattfinden. Hierbei wäre dann auf eine anerkannte normative Theorie, wie bspw. die Deontologie, Tugendethik oder utilitaristische Konzepte, zurückzugreifen, wobei deren Auswahl allerdings wiederum selbst begründungspflichtig ist. ${ }^{717}$ Eine solche Reflexion der vorherrschenden Werte anhand grundlegender, deskriptiv ermittelter sowie ggf. ethisch-normativ begründeter Maßstäbe führt so schließlich zu einer wohlbegründeten bzw. hinreichenden Legitimität. ${ }^{718}$ Die vorausgegangenen Überlegungen zu einer zweistufigen Legitimitätskonzeption verdeutlicht auch nochmals die nachfolgende Abbildung 4.21.

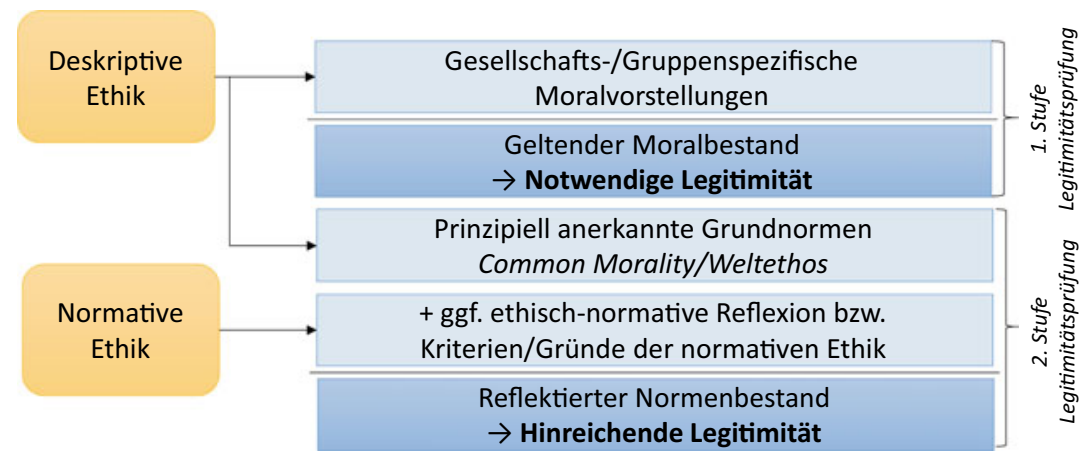

Abbildung 4.21 Zweistufige Konzeption der Legitimitätsevaluation

1131a-1131b). Vgl. im Überblick auch Ladwig, B. (2013) sowie in der historischen Entstehung des Gerechtigkeitsbegriffs auch die Beiträge von Horn, C. (2016) und Koller, P. (2016). In der modernen Forschung hat sich wiederum KÖßLER intensiv mit der Gerechtigkeit der Entlohnung beschäftigt, wobei er insgesamt acht Dimensionen der Gerechtigkeit diskutiert (vgl. Kößler, M. (2001), S. 133).

715 Diese ist im philosophischen Sinne von der Wahrheit (z. B. im korrespondenztheoretischen Sinne) zu unterscheiden. Dagegen stellt Wahrhaftigkeit die „Übereinstimmung der Rede mit dem Gedanken (der Überzeugung)“ (Hartmann, N. (1962), S. 461) dar.

${ }^{716}$ Vgl. Küng, H. (2016), S. 48-49 und S. 82; Küng, H. (2012), S. 35-35; Küng, H. (1997), S. 154-158 sowie insbesondere im Kontext der Globalisierung auch Küng, H. (2001), S. 2538.

717 Vgl. Lingnau, V. / Fuchs, F. (2019), S. 249.

718 Vgl. Lingnau, V. / Fuchs, F. (2019), S. 249-250. 


\subsubsection{Gesamtmodell des Analytischen Frameworks}

Auf diesen Vorüberlegungen aufbauend kann nun das Analytische Framework konzipiert werden. Dieses Framework kann aus einer Metaperspektive, ähnlich wie andere betriebswirtschaftlich diskutierte Maßnahmen im Sinne der kontinuierlichen Verbesserung ${ }^{719}$, als regelmäßig stattfindender, zyklischer Prozess aufgefasst werden. Dieser lässt sich wiederum in insgesamt sechs Phasen einteilen, wie die nachfolgende Abbildung 4.22 aufzeigt.

Hierauf aufbauend ergibt sich detailliert das Ablaufschema des Analytischen Frameworks. Dieses beginnt in einem ersten klassifikatorischen Schritt mit der Ermittlung bzw. Gruppierung der Stakeholder eines Unternehmens. Sind die Stakeholdergruppen definiert, so erfolgt im nächsten Schritt die Ermittlung der Ansprüche der Stakeholder. Hierzu werden nach Anspruchsgruppe zuerst einmal alle ermittelbaren Ansprüche zusammengetragen und ggf. nach Inhalt, Zeit und Ausmaß weiter spezifiziert. Idealerweise kommen hier Verfahren des Diskurses zum Einsatz, wobei in Bezug auf die ,stillen“ Stakeholder wiederum ggf. monologisch-antizipativ vorgegangen werden muss. Darüber hinaus eröffnet aber auch im Kontext der Industrie $4.0 \mathrm{bzw}$. der fortschreitenden Digitalen Transformation der Einsatz moderner Technologien, wie dies u. a. die Methoden des Big Data in Kombination mit Hochleistungsrechnern und Künstlicher Intelligenz darstellen, neue Möglichkeiten. Diese technologischen Grundlagen können genutzt werden, um bspw. im Rahmen semantischer Analysen in sozialen Netzwerken einen weiteren Anhaltspunkt bzw. Wissen über existierende Stakeholderansprüche zu erlangen. ${ }^{720}$ So scheinen vor dem Hintergrund der vorausgegangenen systemtheoretischen Überlegungen materielle sowie immaterielle technische Systeme des Unternehmens zur Generierung eines umfassenderen Bildes der Stakeholderansprüche von zentraler Bedeutung. ${ }^{721}$

${ }^{719}$ Vgl. konzeptionell grundlegend z. B. Benes, G. / Groh, P. E. (2017), S. 186-188; Kostka, C. / Kostka, S. (2017), S. 6-10; Schat, H.-D. (2017), S. 5-8; Marks, T. (2016), S. 4146; Hummel, T. R. / Malorny, C. (2015), S. 39; Rogers, R. E. (2013), S. 15-16; Kopp, R. (1998), S. 27-32; Wahren, H.-K. (1998), S. 7-11; Edosomwan, J. A. (1995), S. 39-42 sowie klassisch Deming, W. E. (2000), S. 23-24.

${ }^{720}$ Im Rahmen des aufgezeigten Wissensbegriffs können auch Maschinen selbständig Wissen erarbeiten. Vgl. hierzu Abschnitt 4.2.4.2 sowie hinsichtlich der systemtheoretischen Überlegungen Abschnitt 4.3.

721 Vgl. auch Lingnau, V. / Fuchs, F. (2019), S. 247. 


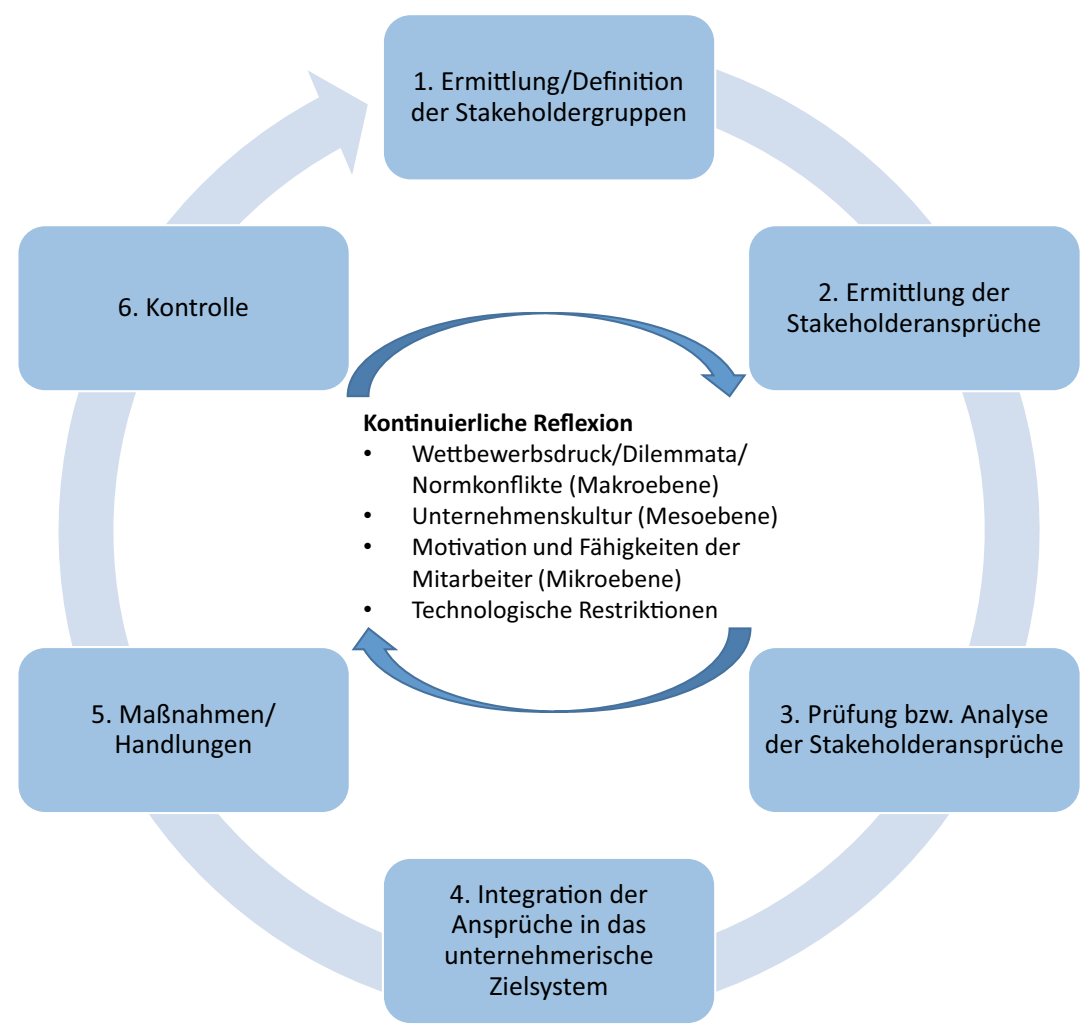

Abbildung 4.22 Ablaufschema des Analytischen Frameworks als kontinuierlicher Verbesserungsprozess

Nachdem die Ansprüche der Stakeholder zusammengetragen wurden, erfolgt eine Prüfung dieser Ansprüche. Dabei können mehrere Prüfkriterien angelegt werden. In einem ersten Schritt erfolgt aus rein deskriptiver Perspektive ein Rekurs auf die faktisch existierenden Regeln bzw. Normen. Dies kann zuerst einmal die klassische rechtliche Zulässigkeit sein, welche allerdings, wie gezeigt wurde, als Compliance-Management noch zu kurz greift, um die Legitimität des Unternehmens zu sichern und im Folgenden daher ausgeklammert sei. ${ }^{722}$

722 In diesem Zusammenhang wären so auch ggf. international konfligierende Rechtsnormen zu evaluieren, wie Abbildung 4.23 aufzeigt. 
Vielmehr sind aus unternehmensethischer Perspektive die Ansprüche gerade auf moralische Zulässigkeit $\mathrm{zu}$ prüfen, welches folglich ebenfalls eine deskriptive Analyse darstellt. In diesem Zusammenhang kann nun wiederum auf die bereits aufgezeigte erste Stufe des Legitimitätsmanagements zurückgegriffen werden. Hierbei sind folglich zuerst einmal die spezifischen Werte, welche in den Wertegemeinschaften, in denen das Unternehmen seine Geschäftstätigkeit vollzieht, von Bedeutung. Bei global tätigen Unternehmen können allerdings, wie zuvor eruiert wurde, durchaus Wertekonflikte auftreten, so dass hier ggf. eine Reflexion und Abwägung dieser Werte vorgenommen werden muss. ${ }^{723}$ In diesem Kontext sei nochmals betont, dass normative Fragestellungen nicht formal letztklärbar sind, so dass im Zweifel auf eine wohlbegründete deskriptive oder auch ethisch-normative Basis zurückgegriffen werden muss, wobei diese Wahl im Sinne des Analytischen Frameworks stets transparent zu machen ist. Diese Grundlagen dienen dann auch zur kritischen Reflexion in der zweiten Stufe der Legitimitätsprüfung. Hierbei kann wiederum in einem ersten Schritt auf prinzipiell anerkannte Grundwerte nach der Common Morality Theory oder dem Weltethos, wie sie sich bspw. auch in der Erklärung der allgemeinen Menschenrechte $^{724}$ der Vereinten Nationen ${ }^{725}$ widerspiegeln, zurückgegriffen werden. Ein solch kritisch reflektierter deskriptiv-ethischer Ansatz erscheint besonders für die Managementpraxis sinnvoll. Darüber hinaus kann schließlich, gerade auch in der Anwendung im akademischen Kontext, zusätzlich zur deskriptiv fundierten auch noch eine ethisch-normative Reflexion zugrunde gelegt werden, wobei an dieser Stelle diverse normative Konzeptionen wie die Deontologie nach $\mathrm{KANT}^{726}$ oder die Tugendethik von ARISTOTELES ${ }^{727}$ Anwendung finden können.

Ist die Prüfung abgeschlossen, so erhält man aus der Gesamtmenge der existierenden Ansprüche die begründeten Stakeholderansprüche. Nun gilt zwar, dass die Ansprüche der Stakeholder prinzipiell legitim bzw. wohlbegründet sein mögen, allerdings können auch diese immer noch zueinander in einem Konkurrenzverhältnis stehen. ${ }^{728}$ Folglich ist in diesem Zusammenhang wiederum eine

${ }^{723}$ Dies verdeutlichen jeweils die Doppelpfeile in Abbildung 4.23.

${ }^{724}$ Diese stellen auch eine zentrale konzeptionelle Grundlage bei Hilpert, K. (2019); Gesang, B. (2016); Brune, G. (2006); Reuter, H.-R. (1999) dar.

725 Vgl. Vereinte Nationen (Hrsg.) (1948).

726 Vgl. Kant, I. (1968b); Kant, I. (1968e).

727 Vgl. Aristoteles (2015).

728 Vgl. hierzu konzeptionell analog Hubig, L. (2009), S. 58, welche sich mit den Beziehungen zwischen Zielen beschäftigt sowie grundlegend die Ausführungen in Abschnitt 4.3.6. 
anschließende Konfliktanalyse der Ansprüche, sowohl innerhalb der Stakeholdergruppen als auch zwischen den Stakeholdergruppen, durchzuführen. Dabei kann mit STEINMANN konstatiert werden, dass „dieses Spannungsverhältnis [.] (natürlich) widerspruchsfrei nicht generell und abstrakt sondern nur konkret situationsbezogen aufgelöst werden [kann]." ${ }^{\text {729 }}$ Falls einige der begründeten Ansprüche nach Inhalt, Zeit und Ausmaß nur partiell erfüllt werden können, ist auch in diesem Falle eine Schlechterstellung faktisch-moralisch (1. Stufe) sowie ggf. auch kritisch deskriptiv bzw. ethisch-normativ fundiert (2. Stufe) zu begründen. ${ }^{730}$ Eine solche mehrstufige Analyse möglicher Konflikte reflektiert auch die Forderung SUCHANEKS, dass die tatsächliche Möglichkeit einer Umsetzung von bestimmten Ansprüchen bzw. realexistierende Handlungsrestriktionen neben der prinzipiellen Begründetheit dieser Ansprüche konzeptionell in der Unternehmensethik zu integrieren sind. ${ }^{731}$

Nach dieser abschließenden Konfliktanalyse der begründeten Ansprüche erhält das Management folglich die Gesamtheit der nach Inhalt, Zeit und Ausmaß zu berücksichtigenden Ansprüche, welche in einem nächsten Schritt in unternehmerische Ziele und Handlungen zu integrieren sind. Dazu erfolgt in einem ersten Schritt eine Prüfung bzw. ggf. Redefinition existierender Unternehmensziele. ${ }^{732}$ Zur Umsetzung dieser Ziele ist dann wiederum auf begründete, $d$. h. insbesondere auch legitime, Mittel zur Erreichung der Ziele zurückzugreifen. Da, wie auch die klassische BWL vielfach hervorhebt, Planung stets sinnvollerweise mit einer Kontrolle der Zielerreichung zu koppeln ist ${ }^{733}$, ist im dritten Schritt auch zu prüfen,

\footnotetext{
729 Steinmann, H. (2011), S. 102. Zudem konstatiert STEINMANN an anderer Stelle: „The decisive point is here that dilemmas, by its very nature, cannot be , solved ' in advance (via planning) through general (organizational) rules; what is needed are, rather, abstract values (as part of the organizational culture) which leave more freedom and flexibility to take into account the specific circumstances relevant for reasonable decision making at the lower echelons of the hierarchy." (Steinmann, H. (2008b), S. 144).

${ }^{730}$ Vgl. hierzu nochmals Lingnau, V. / Fuchs, F. (2019), S. 249-250 sowie Rüegg-Stürm, J. (2003), S. 33.

${ }^{731}$ Vgl. hierzu nochmals die Ausführungen in Abschnitt 3.4.3.2 sowie die Ausführungen in Stuart, I. / Stuart, B. / Pedersen, L. J. T. (2014), S. 52-53, welche ebenfalls zwischen dem Wünschbaren und faktisch Möglichen differenzieren.

732 Hierbei sind diejenigen Ziele gemeint, welche hinreichend bewusst repräsentiert sind und einer konkreten Veränderbarkeit unterliegen. Auf der Mesoebene sind dies folglich insbesondere die s. g. Verhandlungsziele. Vgl. hierzu auch nochmals die Ausführungen in Abschnitt 4.3.6.

733 So gilt klassisch nach WILD: „Planung ohne Kontrolle ist [.] sinnlos, Kontrolle ohne Planung unmöglich." (Wild, J. (1982), S. 44, in der Quelle ebenfalls herv.). Vgl. hierzu auch Behringer, S. (2018), S. 161; Macharzina, K. / Wolf, J. (2018), S. 412-413; Schierenbeck,
} 
inwiefern die vorigen Zielsetzungen erreicht wurden, bzw. ggf. dies bei der nächsten Diskussion der Ziele und möglicher Handlungen zu berücksichtigen. Wichtig ist dabei jedoch auch, dass wo möglich, Indikatoren und Maßgrößen bestimmt werden, um den Erfolg der Unternehmenshandlungen kontinuierlich sicherzustellen. Zudem ist auch hier zu reflektieren, wieweit mögliche Zielzustände zu beeinflussen sind, also hinsichtlich ihrer Controllability durch das Unternehmen eingeschränkt oder diesem in seiner Einwirkungsmöglichkeit gar entzogen sind.

Dabei ist des Weiteren festzustellen, dass bei Integration und Umsetzung der reflektierten Stakeholderansprüche vielfältige Hindernisse auftreten können, welche unternehmensethisch ebenfalls konzeptionell berücksichtigt werden sollten und aus welchen sich ggf. wiederum Implikationen für die Ergreifung von Metahandlungen im Sinne eines potentiellen ,changing the rules of the game“ ergeben. So können, wiederum im Sinne SUCHANEKS, die empirisch existierenden Restriktionen zwar sehr wohl eine Umsetzung der begründeten Ansprüche erschweren. Es gilt daher nach wie vor, dass Unmögliches prinzipiell nicht legitimerweise gefordert werden kann. Allerdings können wiederum sehr wohl in diesem Zusammenhang legitime Ansprüche existieren, die Restriktionen auf legitime Weise zukünftig zu beseitigen. Folglich können sich aus empirisch existierenden Handlungsrestriktionen auf einer Metaebene wiederum Pflichten zu Handlungen ergeben, welche eine Beseitigung oder zumindest Verringerung dieser Restriktionen zum Ziel haben und zur Legitimitätssicherung ergriffen werden müssen, damit zukünftig kein Vorwurf einer illegitimen Unterlassung entsteht.

Als klassische Implementationshindernisse können z. B. im Sinne der wirtschaftsethischen Schule HoMANNS der Wettbewerbsdruck bzw. existierende Dilemmata oder auch Normkonflikte auf der Makroebene genannt werden, welche dann z. B. zu einem Eintreten für eine Änderung (bspw. im Rahmen von Lobbying oder freiwilligen Selbstverpflichtungen von Unternehmensnetzwerken) dieser existierenden Normen führen kann. ${ }^{734}$ Umsetzungsschwierigkeiten können aber auch durch intern vorherrschende Werte, d. h. auf der Mesoebene,

H. / Wöhle, C. B. (2016), S. 122; Bleis, C. (2007), S. 143; Gladen, W. (2003), S. 22; Wall, F. (1999), S. 21; Nuber, W. (1997), S. 125; Böcker, F. (1988), S. 22.

${ }^{734}$ Hierbei ist allerdings, gerade vor dem Hintergrund unvollkommener Märkte und realexistierender unternehmerischer Entscheidungsspielräume, auch zu fragen, inwiefern Unternehmen tatsächlich häufig gezwungen sind, an illegitimen und/oder illegalen Praktiken zu partizipieren. Teilweise, wie dies der Siemens-Skandal um Bestechungsgelder zeigt, müssen solche Praktiken sogar bereits auf strategisch-operativer Ebene als betriebswirtschaftlich fragwürdig eingestuft werden. Vgl. hierzu bspw. die Untersuchung von Berghoff, H. / Rauh, C. (2013) zum Schmiergeldskandal bei Siemens. 
verursacht werden, so dass hier eine Reflexion der Unternehmenskultur notwendig wäre. Auch die Fähigkeiten sowie die Motivation der einzelnen Individuen können sich hinderlich auf die Umsetzung der definierten Ziele auswirken, so dass ebenfalls die Mikroebene reflektiert werden muss. Schließlich ergeben sich ggf. auch technische Restriktionen, welche eine Umsetzung der legitimen Ziele erschweren.

Abschließend sei nochmals hervorgehoben, dass die systematische Reflexion der existierenden Ansprüche der Stakeholder sowie deren Umsetzung einen kontinuierlichen Prozess darstellt, welcher sich folglich nicht auf eine einmalige Durchführung beschränken darf. Wie bereits oben aufgezeigt, können die Überlegungen zum Analytischen Framework auch mit den Entwicklungen im Rahmen der Digitalisierung verknüpft werden. So können die Informationen sowie die zentralen Reflexionsschritte und Analyseergebnisse des Analytischen Frameworks in einem normativen Management Cockpit visualisiert und regelmäßig aktualisiert werden, damit das Management auch über die normative Dimension des unternehmerischen Handelns stets einen transparenten Überblick erhält.

Die nachfolgende Abbildung 4.23 fasst die Struktur des Analytischen Frameworks nochmals anschaulich zusammen.

Die Ermittlung, Analyse und Integration der diversen, existierenden Stakeholderansprüche stellt dabei ein komplexes Problem ${ }^{735}$ dar. Folglich benötigt das Management neben des Einsatzes moderner Technologien vor dem Hintergrund begrenzter kognitiver Ressourcen auch weitere Führungsunterstützung, um die Ansprüche der Stakeholder in unternehmerischen Entscheidungsprozessen hinreichend abwägen sowie integrieren zu können. Hierzu sind regelmäßig s. g. Sekundärwissensträger organisational institutionalisiert, welche als Spezialisten spezifische Anspruchsgruppen in den Fokus rücken und damit Entscheidungen des Managements hinsichtlich ihrer Konsequenzen auf die Anspruchserfüllung einer Stakeholdergruppe reflektieren können. Dieses Konzept und insbesondere auch die spezifischen wirtschaftsethischen Implikationen für das Controlling als einer dieser Sekundärwissensträger sind Gegenstand des nächsten Abschnitts.

735 Zum Begriff der Komplexität vgl. Lingnau, V. / Brenning, M. (2018), S. 146-147; Lingnau, V. / Brenning, M. (2015), S. 457; Gerling, P. G. (2007), S. 23-25 sowie grundlegend Dörner, D. (2002), S. 58-59; Dörner, D. (1983), S. 19-24. 


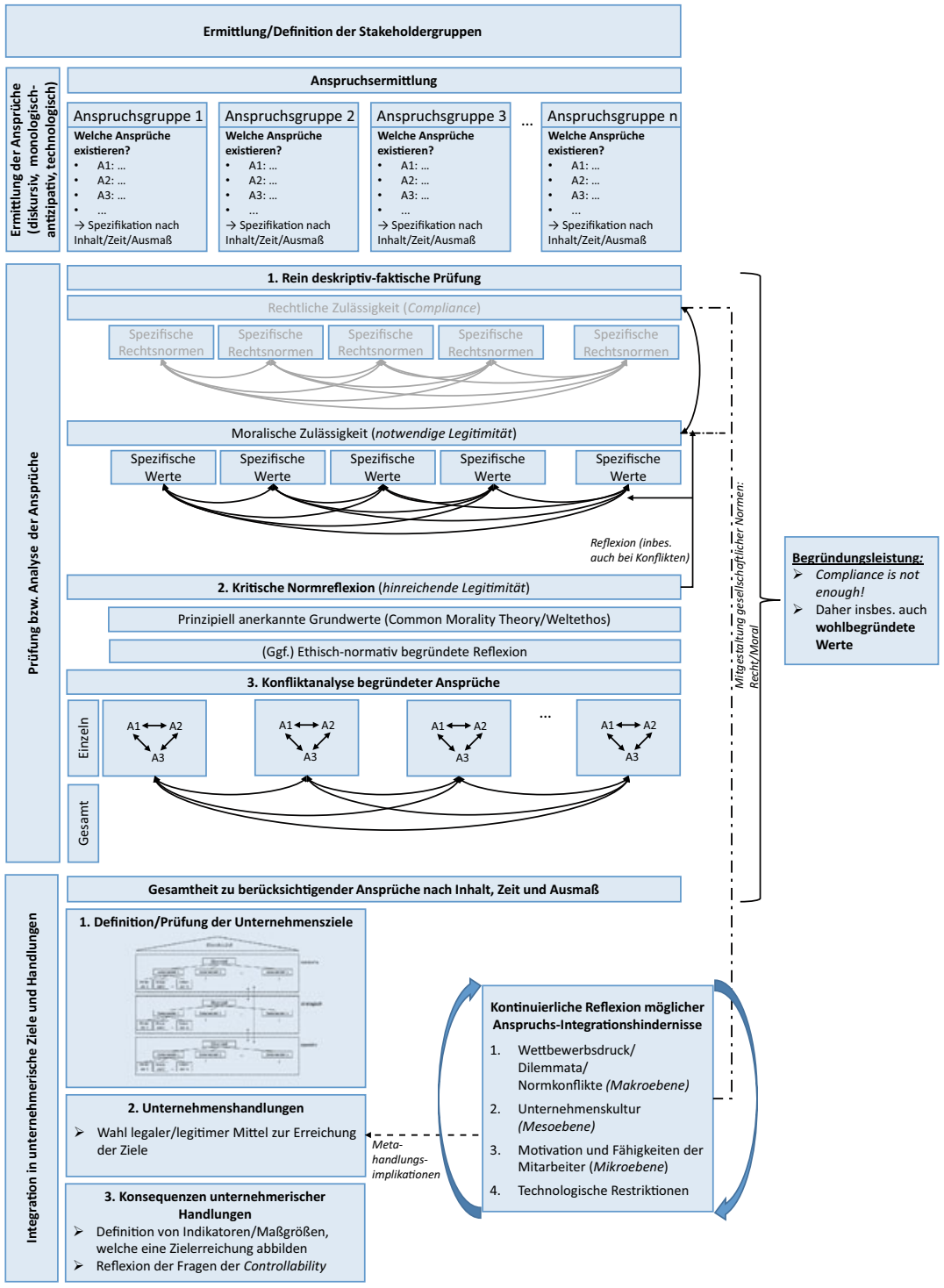

Abbildung 4.23 Das Analytische Framework im Überblick 


\subsection{Unterstützung durch Sekundärwissensträger und Implikationen für das Controlling}

\subsubsection{Grundkonzept der Sekundärwissensträgerschaft und Controllingbegriff}

Der Grundgedanke des Konzepts der Sekundärwissensträgerschaft beruht im Wesentlichen auf einer Abkehr vom Homo oeconomicus-Paradigma der traditionellen, neoklassischen Ökonomik und damit einer Distanzierung von den unrealistischen Annahmen auf kognitiver wie auch motivationaler Seite dieses Ansatzes. Kognitiv unterstellt dieses Konzept dabei zumeist eine infinite Informationsverarbeitungsgeschwindigkeit bzw. eine Vollständigkeit der entscheidungsrelevanten Information in einer gegebenen Entscheidungssituation, welches jedoch evident unrealistisch erscheint. ${ }^{736}$ Eine realistische Reflexion des realen Menschen liefert hinsichtlich der kognitiven Kapazitäten die kognitionswissenschaftliche Forschung, welche die Grenzen hinsichtlich der Aufnahme und Verar-

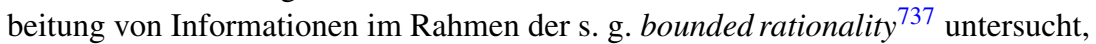
die wiederum maßgeblich auf den Arbeiten HERBERT SIMONS beruht. ${ }^{738}$ So stellte dieser bereits Ende der 1950er Jahre fest: „The capacity of the human mind for formulating and solving complex problems is very small compared with the size of the problems whose solution is required for objectively rational behavior in the real world - or even for a reasonable approximation to such objective rationality. "739 In diesem Sinne scheitert das traditionelle, neoklassische Optimierungsmodell mit

736 Vgl. hierzu auch Lingnau, V. (2001), S. 422.

${ }^{737}$ Der Begriff der bounded rationality wird in der Literatur mit drei zentralen Perspektiven verknüpft: 1. Der Weiterführung der neoklassischen Theorie im Sinne einer mathematischen Optimierung unter Nebenbedingungen, 2. der biased rationality-Forschung in der Tradition von KAHNEMAN und Tversky (vgl. z. B. Kahneman, D. (2012); Kahneman, D. / Tversky, A. (1996); Kahneman, D. / Tversky, A. (1984); Tversky, A. / Kahneman, D. (1974)) sowie 3. die s. g. ökologische Rationalität, welche das Zusammenspiel von realer Umwelt und kognitiven Fähigkeiten betrachtet. Vgl. Gigerenzer, G. / Gaissmaier, W. (2006), S. 333-335. Nach GigERENZER steht dabei letztere am ehesten in der Tradition der Forschung SIMONS. Vgl. Gigerenzer, G. (2004), S. 390-402 und insbes. auch S. 405. Vgl. auch Lingnau, V. (2011b), S. 121-126; Kluge, P. D. / Lingnau, V. (2010), S. 35-39.

738 Dieser wird auch als ,,father" of bounded rationality“ (Gigerenzer, G. / Gaissmaier, W. (2011), S. 452; Gigerenzer, G. / Czerlinski, J. / Martingon, L. (2002), S. 150; Gigerenzer, G. / Todd, P. M. (2001), S. 12) bezeichnet. Vgl. hierzu ebenfalls Lingnau, V. et al. (2012), S. 5-7; Lingnau, V. (2011b), S. 121-126.

${ }^{739}$ Simon, H. A. (1957), S. 198, in der Quelle ebenfalls herv. 
der Prämisse vollständiger Information, denn „our bounded rationality does not permit it. “"740

Übertragen auf den Managementkontext bedeutet dies, dass das Management realistischerweise nicht zugleich alle existierenden Ansprüche kennen, diese Ansprüche auch wiederum begründet ausbalancieren, implementieren und die Anspruchserfüllung regelmäßig überprüfen kann. Es benötigt hierzu die Unterstützung durch Spezialwissen wie dieses in Organisationen durch die Sekundärwissensträger i. d. R. innerhalb bestimmter Abteilungen zur Entscheidungsunterstützung institutionalisiert ist. So fokussiert bspw. das Marketing auf die Ansprüche der Kunden, die Beschaffungsabteilung auf die Ansprüche der Lieferanten, die Personalabteilung auf aktuelles sowie potentielles zukünftiges Personal, die Finanzabteilung bzw. Bilanzbuchhaltung auf die Fremdkapitalgeber und das Controlling schließlich auf die Ansprüche der Eigenkapitalgeber. In diesem Sinne tritt zur Lösung betrieblicher Probleme neben dem primären Führungswissen des Managements das (aus dessen Perspektive) sekundäre Wissen der Sekundärwissensträger hinzu, durch welches das Management fundierte Entscheidungen treffen kann, ohne selbst vollständig über dieses jeweilige anspruchsgruppenspezifische Wissen verfügen zu müssen, wodurch dieses wiederum kognitiv entlastet wird. ${ }^{741}$ In Bezug auf das Analytische Framework bedeutet dies, dass das Management im Wesentlichen auf das anspruchsgruppenspezifische Wissen der Sekundärwissensträger zurückgreift, welche die Ansprüche der jeweiligen Stakeholdergruppe kennen, analysieren bzw. aufbereiten und dem Management zur Verfügung stellen. Diese sind folglich intensiv bei der Anwendung des Analytischen Frameworks einzubinden.

Neben der kognitiven Perspektive, welche sich in der Notwendigkeit einer Entscheidungsunterstützung manifestierte, erscheint aber auch die motivationale Annahme eines unbegrenzten (,harten“) Opportunismus ${ }^{72}$ des Homo oeconomicus in Bezug auf reale Akteure kritikwürdig. So ist im Standardmodell der Ökonomik der Homo oeconomicus vollständig unaffektiert bzw. gleichgültig gegenüber anderen Menschen - außer in dem Falle, in welchem diese Individuen instrumentell zur Erreichung eigener Ziele nützlich erscheinen. In diesem Sinne stellt auch BouldiNG für den Homo oeconomicus fest, dass dieser „,counted every cost and asked for every reward, was never afflicted with mad generosity or uncalculating love, and [.] never acted out of a sense of inner

\footnotetext{
${ }^{740}$ Simon, H. A. (1998), S. 30.

${ }^{741}$ Vgl. grundlegend Lingnau, V. (2010), S. 13-17; Lingnau, V. (2009), S. 21-27; Lingnau, V. (2008b), S. 4-9.

742 Zum Begriff des Opportunismus vgl. Williamson, O. E. (1985), S. 47.
} 
identity and indeed had no inner identity even if he was occasionally affected by carefully calculated considerations of benevolence or malevolence.“743 Auch MANSTETTEN resümiert ähnlich: „Das Wohlbefinden des Homo oeconomicus wird nicht von dem Wohlbefinden oder Elend anderer Menschen beeinflusst. Damit erscheint der Homo oeconomicus als kalkulierender und unersättlicher Egozentriker: Was ihn selbst angeht, so hat er nie genug; was die anderen Menschen angeht, so liegen Anteilnahme und Mitleid, Neid und Missgunst [...] ganz außerhalb seines Horizontes." ${ }^{\text {"744 }}$ Demnach besitzt der Homo oeconomicus keine genuin sozialen Ziele und entspricht damit im Kern dem s. g. Faktor 1Psychopathen. ${ }^{745}$ Wie die empirische Forschung jedoch stabil zeigt, weisen reale Akteure zwar durchaus eigennützige, allerdings auch vielfältige soziale Motive, wie bspw. Altruismus oder Reziprozität, auf. ${ }^{746}$ Die Erkenntnisse der verhaltenswissenschaftlichen Forschung führen damit in der motivationalen Dimension zum Konzept des bounded opportunism ${ }^{747}$, welches zentral für die organisationale Entscheidungsbeeinflussung und damit wiederum höchst relevant bei der Gestaltung von unternehmerischen Anreizsystemen erscheint. ${ }^{748}$ Zusammengefasst ergibt die

743 Boulding, K. E. (1969), S. 10. Diese überaus pessimistischen Annahmen über die vorherrschenden (oder gar ausschließlichen!) Motive von Akteuren hat zu vielfältiger Kritik geführt, so auch zur häufigen Bezeichnung einer solchen Ökonomik als ,dismal science“ (Aldred, J. (2009), S. 11; Fehr, E. / Fischbacher, U. (2002), S. C1) - ein Begriff, welcher ursprünglich auf Thomas CARLYLE zurückgeht (vgl. Brue, S. / Grant, R. (2013), S. 98; Marglin, S. A. (2008), S. 28-30; Levy, D. M. (2002), S. 18-21). Auch AMARTYA SEN kritisiert das Menschenbild des Homo oeconomicus deutlich, welchen er als „,rational fool“ bzw. als „,social moron“ (Sen, A. K. (1977), S. 336) bezeichnet.

${ }^{744}$ Manstetten, R. (2006), S. 42. Vgl. auch Kirchgässner, G. (2013), S. 16; Suchanek, A. / Kerscher, K.-J. (2007), S. 252. So stellt auch HomANS pointiert fest: „The trouble with him was $[\ldots]$ that he was antisocial and materialistic, interested only in money and material goods and ready to sacrifice even his old mother to get them." (Homans, G. C. (1961), S. 79). Ebenso stellen auch HUNT und VITELL fest, dass der ,homo economicus not only maximizes self-interest but does so with opportunistic ,guile‘." (Hunt, S. D. / Vitell, S. (2015), S. 34, in der Quelle ebenfalls herv.).

${ }^{745}$ Vgl. Kühn, C. (2012), S. 125-152 sowie die weiteren Ausführungen in Abschnitt 4.6.2.3. Diese Überlegung hat neuerdings auch NIDA-RUMELIN aufgegriffen. In diesem Sinne konstatiert er: „Der Psychopath ist in der Psychologie das Pendant zum homo oeconomicus in der ökonomischen Theorie.“ (Nida-Rümelin, J. (2020), S. 355, in der Quelle ebenfalls herv.).

${ }^{746}$ Vgl. z. B. die empirischen Forschungsergebnisse bei Falk, A. / Fehr, E. / Fischbacher, U. (2008); Fehr, E. / Schmidt, K. M. (2006); Fehr, E. / Fischbacher, U. (2004); Fehr, E. / Fischbacher, U. (2003); Fehr, E. / Fischbacher, U. (2002); Fischbacher, U. / Gächter, S. / Fehr, E. (2001); Ockenfels, A. (1999); Fehr, E. et al. (1998).

747 Vgl. Lingnau, V. (2010), S. 5.

${ }^{748}$ Vgl. hierzu auch die Ausführungen im nachfolgenden Abschnitt 4.6.2.3. 
realwissenschaftlich ausgerichtete verhaltenswissenschaftliche Forschung folglich zwei Handlungsfelder, welche unter dem Begriff der Führungsunterstützung subsumiert werden können: die aus den Erkenntnissen der bounded rationality abgeleiteten Implikationen hinsichtlich der Entscheidungsunterstützung sowie die Entscheidungsbeeinflussung vor dem Hintergrund des bounded opportunism. ${ }^{749}$

Die vorausgegangenen Überlegungen können nun explizit für das Controlling fruchtbar gemacht werden. So stellt auch SPRINKLE mit Referenz auf DEMSKI und FELTHAM fest, dass die ,two roles for managerial accounting information have been referred to as the decision-facilitating role and the decision-influencing role. ${ }^{\text {750 }}$ Grundsätzlich kann dabei festgehalten werden, dass der Begriff des Controllings in der deutschsprachigen Literatur ${ }^{751}$ überaus schillernd ist und kontrovers diskutiert wird. Einen Überblick liefert dabei FRIEDL, welche die deutschsprachige Controllingforschung in drei große Richtungen gliedert: ${ }^{752}$ Hierzu gehören erstens die informationsorientierten Konzeptionen, welches die Werke von REICHMANN ${ }^{753}$, HAHN und HUNGENBERG ${ }^{754}$ sowie BAuM, COENENBERG und GUNTHER ${ }^{755}$ beinhaltet. Hierbei geht es im Kern um die Bereitstellung von entscheidungsrelevanten Informationen bzw. Wissen, welches jedoch das Problem aufweist, dass dies prinzipiell auch für andere institutionalisierte Funktionsbereiche der Organisation gilt und daher kaum als controllingspezifisch bezeichnet werden kann bzw. keine controllingdiskriminierende Wirkung entfaltet. Darüber hinaus sind die koordinationsorientierten Controllingkonzeptionen $\mathrm{zu}$ nennen, welche insbesondere die Konzeptionen von HORVÁTH ${ }^{756}$ sowie KÜPPER ${ }^{757}$ umfassen. In diesen Ansätzen wird Controlling funktional als Koordination eines mehr oder minder umfangreich ausdifferenzierten, organisationalen Führungssystems aufgefasst. Problematisch ist hierbei unter

\footnotetext{
${ }^{749}$ Vgl. Lingnau, V. (2010), S. 5.

${ }^{750}$ Sprinkle, G. B. (2003), S. 288, in der Quelle ebenfalls herv. Vgl. grundlegend auch Demski, J. S. / Feltham, G. A. (1976), S. 8-12.

${ }^{751}$ Der Terminus des „Controllings“ ist, wenngleich möglicherweise kontraintuitiv, eine Bezeichnung des deutschsprachigen Raums. So stellt auch BECKER pointiert fest: „,Controlling' is a German word." (Becker, A. (1999), S. 2). Im angelsächsischen Raum findet sich dagegen die relativ inhaltsgleiche Bezeichnung als Management- bzw. Managerial Accounting.

752 Vgl. Friedl, B. (2013), S. 95-97. Vgl. auch Lingnau, V. (2010), S. 13.

${ }^{753}$ Vgl. Reichmann, T. / Kißler, M. / Baumöl, U. (2017).

754 Vgl. Hahn, D. / Hungenberg, H. (2001).

755 Vgl. Baum, H.-G. / Coenenberg, A. G. / Günther, T. (2013).

756 Vgl. Horváth, P. / Gleich, R. / Seiter, M. (2020).

${ }^{757}$ Vgl. Küpper, H.-U. et al. (2013).
} 
anderem, dass dies, praktisch unplausibel, eine Weisungsbefugnis gegenüber dem Management voraussetzen würde. Schließlich existiert mit der Konzeption von WEBER und SCHÄFFER ${ }^{758}$ ein rationalitätsorientierter Ansatz, wobei das Controlling als Sicherstellung einer ausreichenden betriebswirtschaftlichen Rationalität der Unternehmensführung aufgefasst wird. Auch hier erscheint jedoch, neben dem per se schillernden Begriff der Rationalität, die Schwierigkeit, ohne Weisungsbefugnis eine wirtschaftliche Rationalität der Führung sicherstellen zu können, konzeptionell problematisch. Damit ist, unbeschadet der Fülle an existierenden Vorschlägen zur Konzeptionalisierung des Controllings im deutschsprachigen Raum, in der Gesamtschau immer noch zu konstatieren, dass bisher keine der Ansätze vor dem Hintergrund einer kritischen konzeptionellen Würdigung vollständig zu überzeugen vermag. ${ }^{759}$

Die Arbeit folgt daher der Konzeption von LingNAU, welcher ein anspruchsgruppenspezifisches Konzept des Controllings mit verhaltenswissenschaftlicher Fundierung entwickelt, das explizit an den obigen kognitionswissenschaftlichen wie motivationalen Überlegungen realer Entscheidungsträger und damit der Bedeutung organisational institutionalisierter Sekundärwissensträger anknüpft. Hiernach ist das Controlling der ,Träger des Wissens über die (formalzielorientierten) Ansprüche ${ }^{760}$ der Eigenkapitalgeber und bringt dieses Wissen unter Berücksichtigung der begrenzten Rationalität und des begrenzten Opportunismus realer Entscheidungsträger in die betrieblichen Problemlösungsprozesse ein. Dies umfasst die mittelbare und unmittelbare Unterstützung des Managements bei Entscheidungen zu Nutzung, Aufbau und Erhalt betrieblicher Erfolgspotentiale, die Unterstützung des Managements bei Entscheidungen zur Beeinflussung von Entscheidungen und die Unterstützung des Managements bei der Begründung dieser Entscheidungen. “761 Das Controlling liefert damit für alle drei Managementebenen (normativ, strategisch und operativ) Wissen zur Entscheidungsunterstützung sowie zur Entscheidungsbeeinflussung, indem es die Ansprüche der Eigenkapitalgeber in die betrieblichen Problemlösungsprozesse einbringt, so z. B. auch bei der Mitgestaltung und kritischen Reflexion unternehmerischer Anreizsysteme. ${ }^{762}$

758 Vgl. Weber, J. / Schäffer, U. (2020). Vgl. auch Schäffer, U. (2004), S. 56-59.

${ }^{759}$ Vgl. Lingnau, V. (2010), S. 14 sowie die Anforderungen an eine Controllingkonzeption bei Winter, P. (2008), S. 9.

${ }^{760}$ Die Formulierung hebt hervor, dass, unbeschadet weiterer möglicher Zieldimensionen, die Ansprüche der Eigenkapitalgeber (gerade auf operativer Ebene) im Kern v. a. auf Rentabilitätsgrößen, d. h. insbesondere die Verzinsung des eingesetzten Kapitals (z. B. Eigenkapitalrendite), fokussiert sind.

${ }^{761}$ Lingnau, V. / Fuchs, F. (2021), S. 14.

762 Vgl. Lingnau, V. / Willenbacher, P. (2013), S. 17-31. 
Im Rahmen dieser Arbeit sind dabei insbesondere einige normative Implikationen für das Controlling von Relevanz. Aus diesem Grunde wird im Folgenden der Fokus auf ethisch relevante Erkenntnisse für die Controllingforschung und -praxis gelegt. Pointiert formuliert geht es hierbei folglich um den Beitrag des Management Accountings zur organisationalen Accountability.

\subsubsection{Ethische Implikationen für das Controlling}

\subsubsection{Konzeptionelle Unterschiede des angelsächsischen und deutschsprachigen Raums}

Eine Betrachtung des Forschungsstands im Kontext ethischer Implikationen für die Controllingforschung und -praxis zeigt, dass einführend, wie auch in den Grundlagen der Wirtschaftsethik, zuerst eine zweigeteilte Betrachtung sinnvoll erscheint, welche zwischen dem englisch- und deutschsprachigen Raum differenziert. Für den angelsächsischen Bereich kann dabei konstatiert werden, dass gerade im Bereich der Accounting Ethics, teilweise sogar explizit mit Rekurs auf die Teildisziplin des Management- bzw. Managerial Accountings ${ }^{763}$, durchaus einige Veröffentlichungen existieren ${ }^{764}$, wobei diese dennoch im Vergleich zu traditionellen Wirtschaftsethiklehrwerken eher eine Nische repräsentieren. ${ }^{765}$ Ähnlich wie im Bereich der Business Ethics kann jedoch auch hier festgestellt werden, dass die Ausführungen zumeist eher pragmatisch bzw. instrumentell ausgerichtet sowie häufig durch einen Mikrofokus gekennzeichnet sind, d. h. es wird auf den Controller bzw. Management Accountant als handelnde Einheit fokussiert und zugleich die Relevanz eines "good conduct" zur Steigerung des wirtschaftlichen Erfolgs anhand von Case Studies ${ }^{766}$ hervorgehoben. Auch für die Controllerpraxis nehmen ethische Fragen eine bedeutsame Rolle ein, wie z. B. die Statuten des britischen Chartered Institute of Management Accountants

\footnotetext{
763 Vgl. z. B. Jackson, S. / Sawyers, R. / Jenkins, G. (2008).

764 Vgl. z. B. Brooks, L. J. / Dunn, P. (2018); Duska, R. F. / Duska, B. S. / Kury, K. W. (2018); Levine, H. J. (2018); Klein, G. (2016a); Stuart, I. / Stuart, B. / Pedersen, L. J. T. (2014); McPhail, K. / Walters, D. (2009); Cheffers, M. / Pakaluk, M. (2007); Baker, R. C. (2006); Mintz, S. M. (1997); Maurice, J. (1996).

765 So stellen auch MCPHAIL und WALTERS fest: „There aren't many textbooks on accounting ethics, at least not in comparison to business ethics textbooks." (McPhail, K. / Walters, D. (2009), S. 3).

766 Dies hebt auch WILLIAMS hervor: „Accounting textbooks invariably now include exercises and cases that are aimed at sensitizing students to the ethical aspects of accounting and auditing." (Williams, P. F. (2010), S. 16).
} 
[CIMA] $^{767}$ oder des US-amerikanischen Institute of Management Accountants $[\mathrm{IMA}]^{768}$ verdeutlichen.

Für den deutschsprachigen Raum lässt sich dagegen, wie auch für die Betriebswirtschaftslehre selbst bereits aufgezeigt, nach wie vor eine vorherrschende konzeptionelle Skepsis hinsichtlich der Beschäftigung mit ethischen Fragestellungen konstatieren. ${ }^{769}$ Folglich findet auch in der deutschsprachigen Controllingforschung eine dezidierte Auseinandersetzung mit controllingethischen Fragen bisher nur in relativ überschaubarem Maße statt. So zeigt die Durchsicht der Literatur, dass lediglich zwei Schulen des Controllings bisher explizit auf ethische Implikationen eingehen - wenngleich dann auch eher kompakt und am Rande der konzeptionellen Überlegungen. Relativ ausführlich beschäftigt sich noch KUPPER mit ethischen Fragestellungen in seinem Controllinglehrwerk, wobei dieser dann - konsistent zur eigenen, koordinationsorientierten Konzeption - die Notwendigkeit der Koordination von Wertvorstellungen der Organisationsmitglieder hervorhebt. ${ }^{770}$ Darüber hinaus gehen auch WEBER und SCHÄFFER basierend auf ihren Überlegungen zur Rationalitätssicherung kurz auf ethische Implikationen ein, wobei sie innerhalb ihrer Konzeption folgerichtig und basierend auf der Annahme von vorherrschenden kognitiven wie motivationalen Divergenzen vom ökonomischen Rationalprinzip ${ }^{771}$ zum Schluss kommen, dass das „Controlling ethisch motiviertes Handeln [...] begrenzen [muss], falls sich dieses nicht rechnet." 772

Insgesamt kann damit nach wie vor konstatiert werden, dass im deutschsprachigen Raum eine intensivere konzeptionelle Beschäftigung mit ethischen Fragen des Controllings noch aussteht, welches gerade auch für die weitere Forschung von besonderer Bedeutung ist. Dabei scheint in einem ersten Schritt klar, dass zur

\footnotetext{
${ }^{767}$ Vgl. Chartered Institute of Management Accountants (Hrsg.) (2020).

768 Vgl. Institute of Management Accountants (Hrsg.) (2017).

${ }^{769} \mathrm{Vgl}$. für die weiteren Überlegungen grundlegend die Ausführungen in Lingnau, V. / Fuchs, F. (2021).

${ }^{770}$ Vgl. Küpper, H.-U. et al. (2013), S. 71-73.

771 So differenzieren WEBER und SCHÄFFER ebenfalls explizit zwischen Könnens- und Wollensproblemen, folgen dann jedoch in der kognitiven Dimension im Wesentlichen der Auffassung der ,,biased rationality“ in der Tradition von KAHNEMAN sowie hinsichtlich der motivationalen Annahmen der eher pessimistischen ökonomischen Standardtheorie. Vgl. Weber, J. / Schäffer, U. (2020), S. 27-28.

772 Weber, J. / Schäffer, U. (2020), S. 60. Hierbei droht jedoch aus ethischer Sicht ein Kategorienfehler, „da Legitimität (ähnlich wie Vertrauen und Reziprozität) und (diskontierte) Zahlungsströme unterschiedlichen Kategorien angehören." (Lingnau, V. / Fuchs, F. (2021), S. 16). Die Berechnung eines trivialen ,Return on Ethics“ ist damit problematisch.
} 
glaubhaften Unterstützung einer ethisch reflektierten Unternehmensführung auch das Controlling mit gutem Beispiel vorangehen muss. Damit hat das Controlling zum einen die Einhaltung gesetzlicher Vorschriften im eigenen Bereich (Compliance), zum anderen aber auch die Legitimität des eigenen Handelns sowie der organisationalen Controlling-Subziele, sicherzustellen. ${ }^{773}$

Darüber hinaus können gerade in Bezug auf die Controllingkonzeption von LINGNAU und in Verknüpfung mit den Überlegungen zur im Rahmen dieser Arbeit entwickelten Unternehmensethikkonzeption noch einige weitere Erkenntnisse der aktuellen Forschung skizziert werden. Orientiert man sich an den beiden Dimensionen der Führungsunterstützung, so können diese Implikationen wie folgt nach den beiden Dimensionen der Entscheidungsunterstützung und Entscheidungsbeeinflussung systematisiert werden.

\subsubsection{Implikationen hinsichtlich der Entscheidungsunterstützung}

Hinsichtlich der Entscheidungsunterstützung ergibt sich im Kern vor allem eine kritische Reflexion des vorherrschenden Controllinginstrumentariums. ${ }^{774}$ So beruhen bspw. einige klassische Controllinginstrumente wie das Capital Asset Pricing Model [CAPM] auf den unrealistischen Prämissen des vollkommenen ${ }^{775}$ Kapital- bzw. Finanzmarktes. ${ }^{776}$ Dabei zeigen jedoch die Finanzkrisen der letzten Jahre, dass nicht nur die Güter- und Arbeitsmärkte als unvollkommen angenommen werden müssen, sondern dies auch klarerweise für die Finanzmärkte gilt - wenngleich traditionell diesen immer noch häufig ein hohes Maß an Vollkommenheit zugeschrieben wird. ${ }^{777}$ Vor dem Hintergrund der jedoch

\footnotetext{
773 Vgl. Lingnau, V. / Fuchs, F. (2021), S. 16.

${ }^{774}$ Vgl. für die weiteren Überlegungen die Ausführungen in Lingnau, V. / Fuchs, F. (2018). Zum Begriff des Controllinginstruments vgl. Jonen, A. / Lingnau, V. (2007), S. 7-9.

${ }^{775} \mathrm{Zu}$ den Eigenschaften dieser Vollkommenheit vgl. Lingnau, V. / Fuchs, F. (2018), S. 6.

${ }^{776}$ In der deutschsprachigen Literatur wird z. T. der Begriff des Finanzmarktes als Überbegriff gewählt, welcher dann z. B. den Kapital-, Devisen- und Geldmarkt umfasst. Der Kapitalmarkt zeichnet sich dabei durch die Möglichkeit der längerfristigen Kapitalanlage, wie bspw. in Aktien oder Anleihen aus. Vgl. Bösch, M. (2019), S. 38; Bieg, H. / Kußmaul, H. / Waschbusch, G. (2016), S. 52; Zantow, R. / Dinauer, J. / Schäffler, C. (2016), S. 47; Laser, J. (2015), S. 7-8; Müller, C. (2015), S. 46; Tolkmitt, V. (2007), S. 20-21.

${ }^{777}$ Die angenommene Nähe zum neoklassischen Ideal wird häufig mit der Homogenität der gehandelten Güter, der Preistransparenz, der Umschlagshäufigkeit sowie des üblicherweise an Börsen getätigten Handels begründet. So postuliert bspw. auch CEZANNE, dass gerade „börsenmäßig organisierte Märkte dem Bild des vollkommenen Marktes ziemlich nahe [kommen].“ (Cezanne, W. (2005), S. 156). Vgl. ähnlich auch Breyer, F. (2020), S. 81. Demgegenüber stellen allerdings SCHIERENBECK und WIEDEMANN fest: „Insbesondere die
} 
evident fehlenden Vollkommenheit dieser Märkte sind die hierauf aufbauenden klassischen Controllinginstrumente folglich einer kritischen Evaluation zu unterziehen und das Management über die Grenzen dieser Instrumente zu unterrichten, da ansonsten die gewonnenen Ergebnisse ,zwar (rechnerisch) genau, aber (inhaltlich) falsch sind“"778, da die Prämissen und damit die realweltlichen Anwendungsgrenzen des Modells verletzt wurden. „Das mit hohem Aufwand berechnete Ergebnis ist dann sowohl genau als auch falsch (Scheingenauigkeit). ${ }^{\prime 779}$

So ist neben der Prämisse einer Vollkommenheit des Marktes beim CAPM bspw. zu problematisieren, dass der zugrunde zu legende risikolose Zinssatz in der Realität schwer eindeutig zu bestimmen ist. Denn, wie die Vergangenheit zeigt, sind gerade die häufig hierzu angesetzten Staatsanleihen auch nicht stets vollkommen risikofrei. Ebenso wäre letztlich auch normativ zu begründen, welche Staatsanleihe bzw. welches Portfolio ${ }^{780}$ zur Ermittlung des risikofreien Investments herangezogen wird. Ebenso kritisch ist beim CAPM die Reduktion der Investitionspräferenzen auf den reinen Erwartungswert der Rendite und deren Standardabweichung zu erachten, welches z. B. die zunehmende Relevanz von genuinen Nachhaltigkeitspräferenzen vernachlässigt. ${ }^{781}$ So stellen auch BEAL, GOYEN und PHILLIPS fest: „One particular type of behavior that has emerged over the last 20 years or so is the desire to invest ethically." ${ }^{782}$ Auch REVELLI und VIVIANI konstatieren in diesem Sinne: „In the past 20 years, socially responsible investing (SRI), which embodies ethical values, environmental protection,

Prämisse des vollkommenen Geld- und Kapitalmarkts ist so in der Praxis nicht haltbar, denn alle Marktteilnehmer, selbst Banken sind stets mehr oder weniger stark gespaltenen Geldund Kapitalmarktzinsen ausgesetzt." (Schierenbeck, H. / Wiedemann, A. (1996), S. 44). Vgl. ähnlich auch Perridon, L. / Steiner, M. / Rathgeber, A. W. (2017), S. 87.

${ }^{778}$ Lingnau, V. / Fuchs, F. (2021), S. 15. Vgl. auch Lingnau, V. / Fuchs, F. (2018), S. 32.

${ }^{779}$ Lingnau, V. (2011b), S. 126.

${ }^{780}$ So z. B. im Rahmen eines s. g. Zero-Beta-Portfolios. Vgl. hierzu Black, F. (1972), S. 445446. Allerdings verbleibt auch hier die empirische Prüfung eines solchen Modells kritisch. Vgl. Kruschwitz, L. / Husmann, S. (2012), S. 245.

${ }^{781}$ Dies zeigt u. a. auch die in der Literatur diskutierte Erweiterung des klassischen Magischen Dreiecks aus Rentabilität, Risiko und Liquidität (vgl. Eichhorn, P. / Merk, J. (2016), S. 214; Ermschel, U. / Möbius, C. / Wengert, H. (2016), S. 34; Bleis, C. (2012), S. 3; Becker, P. (2010), S. 5) zu einem Magischen Viereck, welches auch die Nachhaltigkeitsdimension explizit abbildet. Vgl. Wallis, M. v. / Klein, C. (2015), S. 64; Pinner, W. (2003), S. 28; Friesenbichler, R. / Reithofer, P. (2001), S. 69.

782 Beal, D. J. / Goyen, M. / Phillips, P. (2005), S. 66. 
improved social conditions and good governance, has increasingly attracted the interest of individual and private investors, as well as academics." ${ }^{\text {"783 }}$

In Bezug auf die Repräsentation des Risikos kann darüber hinaus konzeptionell hinterfragt werden, inwiefern die Abbildung als symmetrische Deviation (Standardabweichung bzw. Varianz) vom Erwartungswert tatsächlich das relevante Risiko für den Investor widerspiegelt und nicht vielmehr lediglich die negative Abweichung (,downside risk“), wie dies andere Risikomaße wie bspw. der Value at Risk [VaR] ${ }^{784}$ repräsentieren. So stellte, wie auch LEIPPOLD hervorhebt, bereits MARKOWITZ fest ,that investors should be interested in minimizing downside risk for two reasons: First, only the downside risk is relevant to investors, not the symmetric risk such as variance. Second, under the assumption of normally distributed returns, any downside risk measure would yield the same result as a variance risk measure. Markowitz realizes that if the normality assumption is inappropriate, the downside risk measures become relevant. “785 In diesem Sinne kann auch mit MÜLHAUPT konstatiert werden: „Risiko ist die Gefahr einer negativen Abweichung des tatsächlichen vom erwarteten Wert eines Ereignisses. “786

Auch die Annahme zumindest homogener Erwartungen der Akteure dürfte in der Realität im Vergleich institutioneller und privater Anleger kaum aufrechtzuerhalten sein. Schließlich erscheint aber insbesondere auch die unkritische Fortschreibung von in der Vergangenheit realisierten (Ex-post-)Renditen in die Zukunft bzw. als Ansatz einer wohlbegründeten Ex-ante-Rendite kritisch. So zeigt sich, dass die Renditen der Vergangenheit häufig (auch im Vergleich zur

${ }^{783}$ Revelli, C. / Viviani, J. L. (2015), S. 158. Ähnlich resümieren auch HELLSTEN und MALLIN: „While owners and investors care about [...] their profits, they increasingly also care about other social issues that their investments may one way or another influence [...]." (Hellsten, S. / Mallin, C. (2006), S. 395).

${ }^{784}$ Der Value at Risk gibt bei einem gegebenen Konfidenzniveau an, welcher Wert zu (1- $\left.\alpha\right)$ $\%$ nicht unterschritten wird, bzw. die Wahrscheinlichkeit von $\alpha \%$, dass dieser Wert unterschritten wird. Zur Methode des VaR vgl. Hull, J. (2018), S. 269-271; Kremer, J. (2011), S. 329-333; Alexander, C. (2008), S. 15-17; Jorion, P. (2007), S. 17-20; Saita, F. (2007), S. 2-4 und S. 25-26.

785 Leippold, M. (2015), S. 285. Vgl. grundlegend auch Roy, A. D. (1952).

${ }^{786}$ Mülhaupt, L. (1980), S. 188, in der Quelle fett herv. 
Fremdkapitalverzinsung) sehr hoch ausfallen - und dies in einer Höhe, die standardökonomisch kaum plausibel zu erklären ist (s. g. Equity Premium Puzzle ${ }^{787}$ ), unterstellt man nicht eine absurd hohe Risikoaversion der Investoren. ${ }^{788}$

Darüber hinaus ist auch aus verhaltenswissenschaftlicher Perspektive kritisch festzustellen, dass klassische, kapitalmarktorientierte Ansätze häufig Shareholder als homogene, nutzenmaximierende Einheiten (s. g. Einmütigkeit der Shareholder) auffassen. Gibt man allerdings bereits eine einzige Annahme der klassischen Kapitalmarkttheorie - die Gleichheit von Soll- und Habenzinsen - auf, so kann diese Einmütigkeit nicht mehr gezeigt werden, vielmehr kommt es zu einer Vielzahl an Anspruchsniveaus. Dadurch divergieren die Präferenzen hinsichtlich unternehmerischer Investitionsentscheidungen, womit, je nach anfänglicher Kapitalausstattung und temporaler Konsumpräferenz, wiederum eine für einen Anleger vorteilhafte Investition möglicherweise für einen anderen Anleger als Verlust erscheint. ${ }^{789}$

In diesem Zusammenhang ist auch festzustellen, dass in der verhaltenswissenschaftlichen Tradition SIMONS Investoren nicht als Maximierer, sondern vielmehr als Satisfizierer ${ }^{790}$ aufzufassen sind. ${ }^{791}$ Reale Investoren können aufgrund von Limitationen bezüglich vorhandenen Wissens, aber auch in Bezug auf die Möglichkeiten einer umfassenden Suche von Informationen, keine Globaloptimierung ihres Portfolios durchführen. Sie brechen daher die heuristische Suche nach superioren Investitionsentscheidungen bei Erreichung eines zuvor

\footnotetext{
${ }^{787}$ Vgl. Mehra, R. / Prescott, E. C. (1985), S. 145-146 sowie den aktualisierten Überblick bei Mehra, R. (2006). Das Equity Premium Puzzle tritt zudem global stabil auf. Vgl. hierzu Forbes, W. (2009), S. 269-270; Erbas, S. N. / Mirakhor, A. (2007), S. 8.

788 So müsste man unter Zugrundelegung der traditionellen, neoklassischen Nutzentheorie davon ausgehen, dass ein Entscheidungsträger zwischen einer 50-prozentigen Wahrscheinlichkeit auf einen Gewinn von $\$ 50.000$ bzw. $\$ 100.000$ und einem sicheren Gewinn von ca. \$52.145 indifferent wäre. Der Berechnung liegt dabei die für Prognosezwecke angepasste Risikoaversion von A = 17,5 nach den Daten von Mehra, R. / Prescott, E. C. (1985) zugrunde. Vgl. Mankiw, N. G. / Zeldes, S. P. (1991), S. 103-105.

${ }^{789}$ Vgl. hierzu auch detailliert die Diskussion in Lingnau, V. / Fuchs, F. (2018), S. 11-14.

${ }^{790}$ Die Bezeichnung der „Satisfizierung“ bzw. des „,satisficing“ stellt ein von HERBERT SIMON geprägtes Kofferwort dar, welches sich aus den Termini „Satisfaction“ und „Sufficiency“ bzw. „satisfy“ and „suffice“ zusammensetzt. Es geht hierbei folglich um die Zufriedenheit mit einer ausreichenden Erfüllung eines ex ante definierten Anspruchsniveaus. Vgl. Neuman, W. R. (2016), S. 110; Hinton, A. (2015), S. 62; Manktelow, K. I. (2012), S. 220. ${ }^{791}$ In diesem Sinne stellt SIMON fest: „Evidently, organisms adapt well enough to , satisfice“; they do not, in general, ,optimize."“ (Simon, H. A. (1956), S. 129).
} 
als hinreichend definierten Satisfizierungsniveaus $\mathrm{ab}^{792}$ und treffen basierend auf diesem Wissensstand ihre Investitionsentscheidung. ${ }^{793}$ Vor diesem Hintergrund kann schließlich mit KRUSCHWITZ und HuSMANN „die Schlussfolgerung gezogen werden, dass das CAPM heute nur noch geringe empirische Unterstützung findet." ${ }^{\text {"794 }}$ Obschon durchaus weiterer Forschungsbedarf hinsichtlich der Frage besteht, in welchen Kontexten Heuristiken erfolgversprechend und traditionellen Instrumenten bezüglich ihres Ergebnisses ebenbürtig, d. h. „ökologisch rational“795, sind, sei hier nur auf die existierende empirische Evidenz verwiesen, nach der relativ einfache Heuristiken durchaus zu „guten“ bzw. vergleichbaren (und z. T. sogar superioren) Ergebnissen in Bezug zu traditionellen, mathematischen Optimierungsmodellen führen können. ${ }^{796}$ Heuristiken sind nach GIGERENZER hinsichtlich der kognitiven Beanspruchung des Anwenders schnell

792 Dabei sei angemerkt, dass bei der Suche auftretende Cues auch zu einer Anpassung des Anspruchsniveaus führen können. So stellen auch GoODIE ET AL. fest: „,[A]spiration levels [...] may be gradually adjusted if they become too lax or binding a constraint." (Goodie, A. S. et al. (2001), S. 351). Vgl. auch Gigerenzer, G. / Brighton, H. (2009), S. 108 sowie die Ausführungen zur ,,aspiration adaptation theory“ in Selten, R. (2001), S. 18-24 und grundlegend Selten, R. (1998); Sauermann, H. / Selten, R. (1962).

793 In diesem Sinne stellt SIMON fest: ,Two concepts are central [...]: search and satisficing.“ (Simon, H. A. (1979), S. 502, in der Quelle ebenfalls herv). Vgl. auch Simon, H. A. (1955), S. 108-110.

${ }^{794}$ Kruschwitz, L. / Husmann, S. (2012), S. 250.

${ }^{795}$ Es sei darauf hingewiesen, dass der Begriff der „Ökologie“ hier nicht im klassischen Nachhaltigkeitsdiskurs steht. Vielmehr beschreibt das Prinzip der ökologischen Rationalität im heuristischen Kontext, dass der Erfolg (bzw. die Rationalitätsevaluation) einer Heuristik an eine bestimmte Umwelt gekoppelt ist. Vgl. hierzu Todd, P. M. / Gigerenzer, G. (2012), S. 14-16; Gigerenzer, G. / Gaissmaier, W. (2006), S. 332 und S. 342; Gigerenzer, G. / Todd, P. M. (2001), S. 13. So subsumieren GIGERENZER und GAISSMAIER wie folgt: „Ökologische Rationalität impliziert, dass eine Heuristik nicht an sich gut oder schlecht, rational oder irrational ist, sondern nur in Bezug auf eine bestimmte Umwelt, indem sie sich bestimmte Strukturen einer Umwelt zu Nutze macht oder diese verändert." (Gigerenzer, G. / Gaissmaier, W. (2006), S. 332).

${ }^{796}$ Vgl. Gigerenzer, G. / Brighton, H. (2009), S. 110-116; Goldstein, D. G. / Gigerenzer, G. (2009), S. 760 und S. 764. Klassisch wäre hier z. B. die 1/N-Heuristik zu nennen, d. h. einer Allokation des zu investierenden Vermögens von $1 / \mathrm{N}$ (d. h. Gleichverteilung) auf $\mathrm{N}$ Investitionsalternativen. Hierbei konnte empirisch gezeigt werden, dass diese sogar bessere Ergebnisse liefert als 14 deutlich komplexere Investitionsmodelle, welches u. a. sogar ein nobelpreisgekröntes Modell umfasste. Vgl. hierzu DeMiguel, V. / Garlappi, L. / Uppal, R. (2009), S. 1930-1944. So stellen die Autoren insgesamt fest: „Of the 14 models we evaluate across seven empirical datasets, none is consistently better than the $1 / \mathrm{N}$ rule in terms of Sharpe ratio, certainty-equivalent return, or turnover [...].“ (DeMiguel, V. / Garlappi, L. / Uppal, R. (2009), S. 1915). 
bzw. sparsam, robust gegenüber neuen Situationen sowie transparent, d. h. leicht nachzuvollziehen. ${ }^{797}$

Ein vielversprechender Ansatz zur Evaluation der realexistierenden, aber auch mit klassischen Instrumenten wie dem CAPM berechneten Eigenkapitalrenditen, stellt die vom Autor dieser Arbeit mitentwickelte $\mathrm{FK}^{+}$-Heuristik dar. ${ }^{798}$ Grundgedanke ist hierbei, die Eigenkapitalrendite über die Fremdkapitalverzinsung zu berechnen. Geht man davon aus, dass spätestens seit der Regulierung durch Basel II und III ${ }^{799}$ alle grundlegenden unternehmerischen Geschäftsrisiken im Fremdkapitalzinssatz berücksichtigt $\operatorname{sind}^{800}$, so kann die anzusetzende Eigenkapitalrendite prinzipiell auch als eigenkapitalgeberspezifischer Risikozuschlag zu diesen bereits im Fremdkapitalzins eingepreisten grundlegenden Risiken ermittelt werden. Formal kann diese Beziehung mathematisch wie folgt formuliert werden:

$$
r_{E K}=r_{F K}+\text { Risikoprämie }_{E K}
$$

Als zusätzliche, eigenkapitalgeberspezifische Zusatzrisiken sind insbesondere drei Risikodimensionen zu berücksichtigen. Dies ist erstens die Nachrangigkeit im Insolvenzfall (Nachrangigkeitszuschlag), da das Eigenkapital im Falle der Insolvenz zuerst zur Befriedigung offener Stakeholderansprüche genutzt wird. Ferner ist auch zweitens ein empirisch oftmals festzustellendes größeres negatives Abweichungsrisiko der Rendite (Renditerisikozuschlag) zu nennen. Schließlich können als dritte Risikokategorie noch andere, nicht im Fremdkapitalkostensatz abgedeckte Risiken (Restrisikozuschlag) genannt werden, wie

${ }^{797}$ Vgl. Gigerenzer, G. (2007b), S. 63-64; Gigerenzer, G. / Gaissmaier, W. (2006), S. 332. Analog zu den kognitiven Heuristiken wird die Performanz schneller, sparsamer, robuster sowie transparenter Heuristiken häufig anhand der Blickheuristik zum Auffangen eines im Schwerefeld der Erde parabelförmig fliegenden Balls beim schiefen Wurf verdeutlicht. Vgl. hierzu Gigerenzer, G. (2010), S. 21-22; Gigerenzer, G. (2007a), S. 17-21; Gigerenzer, G. / Gaissmaier, W. (2006), S. 330-332.

${ }^{798}$ Vgl. hierzu sowie die folgenden Ausführungen detailliert Lingnau, V. / Fuchs, F. (2018), S. 42-46.

${ }^{799} \mathrm{Zu}$ den Implikationen von Basel II vgl. Jonen, A. / Lingnau, V. (2004), S. 9-15. Die Schlussfolgerungen gelten dabei noch in verstärktem Maße für Basel III. Für die Änderungen in Basel III vgl. zusammenfassend Hartmann-Wendels, T. (2013), S. 75-85. Zur Möglichkeit einer Risikoselbstbewertung (Self-Rater), insbesondere für kleine oder mittelständische Unternehmen, vgl. Hoogen, M. / Jonen, A. / Lingnau, V. (2006), S. 9-13.

${ }^{800}$ Dies kann durch den Marktmechanismus auch für die direkte unternehmerische Ausgabe von Schuldverschreibungen (Corporate Bonds) angenommen werden. Vgl. Lingnau, V. / Fuchs, F. (2018), S. 43. 
bspw. das in bestimmten Regionen möglicherweise existierende Risiko einer entschädigungslosen Verstaatlichung. ${ }^{801}$

Darüber hinaus hat die Literatur auch weitere Heuristiken, welche als Controllinginstrumentarium hilfreich sein könnten, hervorgebracht. ${ }^{802}$ Beispielhaft wäre hier ein Modell zur vereinfachten Quantifizierung von Realoptionen bei LUEHRMAN $^{803}$ zu nennen, oder das Entscheidungsmodell von SLATER, REDDY und ZWIRLEIN ${ }^{804}$, welches sowohl die Attraktivität eines risikoadjustierten Kapitalwerts als auch das Potential einer Realoption bewertet und beide Dimensionen dann in eine 9-Feldermatrix integriert.

Insgesamt ist vor dem aufgezeigten Hintergrund einer realwissenschaftlich fundierten Entscheidungsunterstützung für das Controlling die Schlussfolgerung zu ziehen, dass traditionelle Instrumente einer kritischen Reflexion hinsichtlich der ihnen zugrunde liegenden Prämissen zu unterziehen sind, um Instrumente einzusetzen, welche ein ,höheres Potential an ökologischer Rationalität auf[weisen]. " ${ }^{\circ 05}$ So sind Ergebnisse, welche auf reinen Idealprämissen basieren, nicht unbedingt geeignet, um praxisbezogene Problemstellungen zu unterstützen. Dies gilt insbesondere auch für die normative Begründungsfunktion des Managements, wenn dieses seine Entscheidungen nicht auf realwissenschaftlich überzeugenden Instrumenten fundiert. Im Gegenteil droht, z. B. gerade durch den Ansatz systematisch zu hoher Renditeforderungen der Shareholder, wie auch der öffentliche Diskurs zeigt, sogar eine gesellschaftliche Delegitimation eines Unternehmens, weshalb die Relevanz einer kritischen Reflexion der zur Entscheidungsunterstützung des Managements eingesetzten Controllinginstrumente nochmals hervorgehoben sei.

Abschließend sei auch noch im Sinne der kritischen Methodenkompetenz eine expansive, unkritische Anwendung des Controllinginstrumentariums als alleiniger oder zumindest dominanter Entscheidungsmaßstab für das Management betrachtet. So heben zwar die Vertreter des traditionellen Shareholder Value-Ansatzes hervor, dass eine Maximierung des Shareholder Values als Residualgröße nur legitim sei, da diese lediglich nach Befriedigung aller anderen Ansprüche verbleibe und zudem die Eigenkapitalgeber auch das Hauptrisiko des Untergangs des Unternehmens tragen würden. Diese Feststellungen gelten allerdings lediglich

${ }^{801}$ Vgl. Lingnau, V. / Fuchs, F. (2018), S. 44. Als erste Abschätzung konnte für DAXUnternehmen ein approximativer Zuschlag von ca. 10,5\% ermittelt werden.

802 Vgl. Lingnau, V. (2011b), S. 126-129.

${ }^{803}$ Vgl. Luehrman, T. A. (1998), S. 55-64.

${ }^{804}$ Vgl. Slater, S. F. / Reddy, V. K. / Zwirlein, T. J. (1998), S. 453-457.

${ }^{805}$ Lingnau, V. (2011b), S. 129. 
unter den Bedingungen vollkommener Märkte (und hier: insbesondere vollständiger Verträge). In der Realität sind, wie aufgezeigt, weder die entsprechenden Märkte vollkommen noch die Beiträge der Stakeholder vollständig ex ante vertraglich fixiert bzw. rechtlich abgesichert. Dies zeigen auch etliche Unternehmensschieflagen, in welchen Mitarbeiter, aber auch Fremdkapitalgeber über die ex ante vertraglich spezifizierten Beiträge durch Lohnverzicht oder Stundung ebenfalls einen weiteren Beitrag zur Sicherung bzw. Sanierung des Unternehmens leisten. In diesem Sinne ist es folglich zentral, dass das Controlling das Management auch auf die Nicht-Komplementarität der Eigenkapitalwertmaximierung mit den Ansprüchen anderer Stakeholder und damit die Grenzen seiner Entscheidungsunterstützungsfunktion hinweist, wodurch Managemententscheidungen, wie auch der Stakeholderansatz nahelegt, nicht nur basierend auf den Ergebnissen der Instrumente des Controllings getroffen werden dürfen.

Nachdem nun die ethischen Implikationen für das Controlling in der Dimension der Entscheidungsunterstützung herausgearbeitet wurden, befasst sich die Arbeit im nächsten Unterkapitel noch mit den controllingrelevanten Erkenntnissen hinsichtlich der Entscheidungsbeeinflussung.

\subsubsection{Implikationen hinsichtlich der Entscheidungsbeeinflussung}

Im Rahmen der Entscheidungsbeeinflussung nimmt das Controlling eine zentrale Rolle bei der Mitgestaltung unternehmerischer Incentives ein, insbesondere seit dem Aufkommen wertorientierter Anreizsysteme ${ }^{806}$ und der diesen zugrunde liegenden Performancemessung. ${ }^{807}$ So stellt z. B. auch KRAMER fest: „Innerhalb des Unternehmens werden Incentives häufig gemeinsam von der Geschäftsführung und dem Controlling entwickelt, verabschiedet und implementiert." ${ }^{808}$ Ebenso konstatieren SCHÄFFER und WEBER: „Während Anreize für Manager noch vor zehn oder zwanzig Jahren nur in den seltensten Fällen ein Thema waren, das Controller in Unternehmen aktiv mitgestalten konnten, ist dies heute zumindest in großen Unternehmen sehr häufig der Fall.“"809

Dabei erscheint allerdings eine kritische Untersuchung der Konsequenzen traditioneller monetärer Individualincentives bedeutsam, denn diese können zu einem negativen Selektionseffekt führen. In diesem Kontext untersuchte KUHN

\footnotetext{
${ }^{806}$ Vgl. hierzu im Überblick auch Weber, J. et al. (2017), S. 157-170.

807 Vgl. Lingnau, V. / Willenbacher, P. (2013), S. 23-31.

${ }^{808}$ Kramer, S. (2011), S. 20.

${ }^{809}$ Schäffer, U. / Weber, J. (2011), S. 1. Vgl. auch Willenbacher, P. (2017), S. 114-115; Lingnau, V. / Willenbacher, P. (2013), S. 1.
} 
erstmalig auf konzeptioneller Ebene den Zusammenhang zwischen dem klassischen Menschenbild der Ökonomik (Homo oeconomicus) und traditionellen materiellen Incentives. Sie stellt dabei zum einen fest, dass Anreizsystemen stets ein bestimmtes Menschenbild als prototypisch unterstelltes Verhaltensmuster zugrunde liegt. Zum anderen wirken dann Anreizsysteme hierauf aufbauend durch ihre Belohnungsfunktion als Filter, denn Individuen, welche dem Anreizsystem entsprechend handeln, werden belohnt und steigen dadurch als leistungsstark evaluierte Mitarbeiter schneller in der organisationalen Hierarchie auf. ${ }^{810}$ Klassische Anreizsysteme beruhen dabei auf dem Homo oeconomicus-Paradigma, d. h. der Annahme, dass Mitarbeiter vor allem durch extrinsische Anreize motiviert werden (müssen!) und ansonsten durch opportunistische Arbeitsvermeidung bzw. „Arbeitsleid“ gekennzeichnet sind ${ }^{811}$, welches durch das Anreizsystem so gemildert werden soll, dass die opportunistische Eigennutzverfolgung zur (individuell quasi „unbeabsichtigten“) Komplementarität mit den unternehmerischen Zielen führt. Die Zugrundelegung des Homo oeconomicus als Set von Verhaltensannahmen stellt sich jedoch bei genauerer Betrachtung als überaus problematisch dar. So zeigt eine Analyse der Verhaltenseigenschaften des Homo oeconomicusModells, dass dieses im Kern die Eigenschaften eines s. g. Faktor 1-Psychopathen aufweist ${ }^{812}$, der auch als „funktionaler“ bzw. „erfolgreicher“ Psychopath ${ }^{813}$

${ }^{810}$ Vgl. Kühn, C. (2012), S. 170-175.

${ }^{811}$ Vgl. Willenbacher, P. (2017), S. 213; Landes, M. / Spörrle, M. / Steiner, E. (2013), S. 74; Pieroth, G. (2013), S. 99-100; Kühn, C. (2012), S. 167; Aßländer, M. S. (2009), S. 277; Süßmair, A. (2000), S. 173; Kugler, F. (1994), S. 13-14; Haubl, R. et al. (1986), S. 133. Dies führt im Rahmen der Prinzipal-Agenten-Theorie wiederum zur Annahme der „Drückebergerei“ bzw. „Shirking“ auf Seiten des Arbeitnehmers (Agenten) im Sinne der Minimierungsregel des formalen ökonomischen Prinzips. Vgl. Küpper, H.-U. et al. (2013), S. 102-104; Heyd, R. / Beyer, M. (2011), S. 33; Mühlenkamp, H. (2007), S. 63; Scherm, E. / Pietsch, G. (2007), S. 57-58; Smith, S. W. (2003), S. 99; Kleine, A. (1996), S. 26.

${ }^{812}$ Vgl. Kühn, C. (2012), S. 126-152. So stellt KUHN schließlich fest: „Beim homo oeconomicus handelt es sich um einen homo psychopathicus." (Kühn, C. (2012), S. 171, in der Quelle ebenfalls herv.).

${ }^{813}$ Vgl. z. B. Forster, N. / Lund, D. W. (2018); Lilienfeld, S. O. / Watts, A. L. / Smith, S. F. (2015), S. 298; Ramirez, E. (2015), S. 330; Dutton, K. (2012), S. 131; Mullins-Sweatt, S. N. et al. (2010), S. 554; Fairholm, G. W. (1994), S. 216. 
sowie vielfach als „Corporate Psychopath“ bzw. „Unternehmenspsychopath“814 bezeichnet wird.

Der Begriff der Psychopathie selbst weist eine lange Historie und in der Begriffsetymologie eine noch längere Zeit vorherrschende große Begriffsbreite auf. ${ }^{815} \mathrm{Im}$ alltagssprachlichen Begriffsverständnis besitzt dieser Begriff zudem häufig eine Konnotation mit Gewalttätigkeit, welche jedoch, wie die weiteren Ausführungen zeigen, nicht notwendig für das moderne Verständnis des Konstrukts der Psychopathie ist. ${ }^{816}$ Erste fachwissenschaftliche Präzisierungsversuche lassen sich dabei ab dem 19. Jahrhundert auffinden, so z. B. bei PINELS Konzept der „manie sans délire“ ${ }^{417}$, welches bereits die Klarheit, mit welcher Psychopathen normativ deviant handeln können, beinhaltet. Als wegweisend für die moderne Psychopathieforschung sind besonders die Überlegungen von CLECKLEY ab den 1940er Jahren zu nennen ${ }^{818}$ sowie die hierauf aufbauende Systematisierung psychopathischer Eigenschaften in einer Merkmalsliste ${ }^{819}$ zur Messung

814 Vgl. z. B. Brooks, N. / Fritzon, K. / Watt, B. (2020), S. 20; Boddy, C. R. P. (2017), S. 141; Furtner, M. (2017), S. 24; Lingnau, V. / Fuchs, F. / Dehne-Niemann, T. E. (2017), S. 1195; Scholz, C. (2014), S. 939; Babiak, P. / Neumann, C. S. / Hare, R. D. (2010), S. 174. Vgl. ebenfalls Babiak, P. (1995), S. 171, welcher diese Individuen auch als ,industrial psychopaths“ sowie Boddy, C. R. P. (2006), S. 1461, der diese auch als „organisational psychopaths“ bezeichnet.

${ }^{815}$ Vgl. hierzu im Überblick die Ausführungen bei Babiak, P. / Hare, R. D. (2019), S. 22-25; Abel, T. / Dulz, B. (2017), S. 11-12; Kühn, C. (2012), S. 17-28; Skeem, J. L. et al. (2011), S. 99-100; Hare, R. D. (1996), S. 27. Etymologisch geht die Bezeichnung auf die griechi-

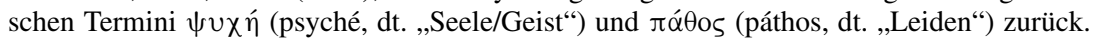
Vgl. Poeppl, T. B. et al. (2019), S. 463; Forster, N. / Lund, D. W. (2018), S. 22; Clarke, J. (2005), S. 41.

${ }^{816}$ Vgl. hierzu auch Skeem, J. L. et al. (2011), S. 97. Vgl. zum Begriff des Konstruktes in diesem Zusammenhang auch Irtelli, F. / Vincenti, E. (2017), S. 188-189.

${ }^{817}$ Vgl. Pinel, P. (1809), S. 157-160.

${ }^{818}$ Vgl. Cleckley, H. (1988), S. 337-364.

${ }^{819}$ Dies erscheint auch notwendig, da sich Psychopathie als Syndrom aus einer Vielzahl von Krankheitszeichen bzw. Symptomen konstituiert. So stellt auch HARE fest: „Psychopathy is a syndrome - a cluster of related symptoms." (Hare, R. D. (1999), S. 34, in der Quelle ebenfalls herv.). Vgl. auch Hare, R. D. (1998), S. 115. Psychopathie stellt dabei eine gravierende Persönlichkeitsstörung dar. Sie wird mittlerweile häufig als Teil der s. g. Dunklen Triade der Charaktereigenschaften eines Menschen aufgefasst, welche neben der Psychopathie auch Narzissmus und Machiavellismus umfasst. Vgl. Paulhus, D. L. / Williams, K. M. (2002), S. 559-560. 
von Psychopathie nach HARE ${ }^{820}$, welche in der Literatur vielfach als ,gold standard" 821 der modernen Psychopathieforschung aufgefasst wird. Die von HARE entwickelten 20 Items $^{822}$ können anhand empirischer faktoranalytischer Untersuchungen wiederum in mehrere Faktoren verdichtet werden. Als klassisch kann dabei die Zweifaktorenstruktur der Psychopathie erachtet werden ${ }^{823}$, welche auch zur Klassifikation des im organisationalen Kontext ,erfolgreichen“ (Faktor 1-) Psychopathen herangezogen werden kann sowie dessen Bezeichnung erklärt. Der erste Faktor beschreibt hierbei die Beeinträchtigung der affektualen Ebene und

${ }^{820}$ Diese wurde von HARE ursprünglich als Psychopathy Checklist [PCL] bezeichnet und später in ihrer heute noch gültigen Form als Psychopathy Checklist - Revised [PCL-R] nochmals verbessert veröffentlicht. Vgl. grundlegend Hare, R. D. (2003). Darüber hinaus finden sich auch Abwandlungen der PCL-R wie beispielsweise eine verkürzte Screeningversion (vgl. Hare, R. D. (1998), S. 103; Hare, R. D. (1996), S. 31-32; Babiak, P. (1995), S. 174) sowie Adaptionen speziell für die Messung von Psychopathie bei Jugendlichen bzw. jungen Erwachsenen (vgl. Hare, R. D. (1996), S. 32-33). Des Weiteren existieren auch weitere bekannte Skalen wie bspw. das Psychopathic Personality Inventory - Revised [PPI-R], welches im Rahmen der Selbstbeurteilung, so z. B. im Surveykontext, zum Einsatz kommt. Vgl. grundlegend Lilienfeld, S. O. / Widows, M. R. (2005).

${ }^{821}$ Vgl. Fritzon, K. / Brooks, N. / Croom, S. (2020), S. 137; Externbrink, K. / Keil, M. (2018), S. 69; Heinzen, H. et al. (2014), S. 108; Lynam, D. R. (2011), S. 272; Boddy, C. R. P. / Ladyshewsky, R. K. / Galvin, P. (2010a), S. 5; Boddy, C. R. P. / Ladyshewsky, R. K. / Galvin, P. (2010b), S. 122; Falkenbach, D. (2007), S. 227; Acheson, S. K. (2005), S. 430; Westen, D. / Weinberger, J. (2004), S. 599.

${ }^{822}$ Die Items können dabei jeweils einen Wert zwischen 0 (trifft gar nicht zu), 1 (trifft teilweise zu bzw. unsicher) und 2 (trifft voll zu) annehmen, so dass ein Individuum einen Gesamtscore von 0 bis 40 aufweist. Vgl. Hare, R. D. / Neumann, C. S. (2008), S. 219; Hare, R. D. (1996), S. 30. Die PCL-R ist folglich ein graduelles Maß zur Messung des Ausmaßes an Psychopathie, wobei häufig dennoch ein Wert von 30 (in den USA) bzw. 25 (in Europa) als Cut-Off herangezogen wird, um ein Individuum als (klinischen) Psychopathen zu klassifizieren. Vgl. Babiak, P. / Hare, R. D. (2019), S. 25-26; Hare, R. D. / Neumann, C. S. / Mokros, A. (2018), S. 46; Hare, R. D. (1996), S. 36.

${ }^{823}$ Darüber hinaus hat die Forschung auch weitere Systematisierungen anhand faktoranalytischer Untersuchungen erarbeitet, so z. B. das Dreifaktorenmodell von COOKE und MICHIE (vgl. Cooke, D. J. / Michie, C. (2001), S. 176). In einer neueren Systematisierung haben HARE und NEUMANN zudem ein Vierfaktorenmodell entwickelt (vgl. Hare, R. D. / Neumann, C. S. (2005), S. 57-60, vgl. auch Babiak, P. / Hare, R. D. (2019), S. 26-27). Dabei können jedoch die ersten beiden Faktoren (Callous Affect, Interpersonal Manipulation) wieder zum klassischen Faktor 1 und die beiden letzten Faktoren (Erratic Lifestyle, Antisocial Behaviour) zum traditionellen Faktor 2 reaggregiert werden. Vgl. Dehne-Niemann, J. / DehneNiemann, T. E. / Lingnau, V. (2020), S. 48-49; Lingnau, V. / Dehne-Niemann, T. E. (2015), S. 73-75; Kühn, C. (2012), S. 41-42; Hare, R. D. / Neumann, C. S. (2008), S. 219-220. 
umfasst sowohl die Empathielosigkeit bzw. Kaltherzigkeit ${ }^{824}$ eines Individuums als auch die Tendenzen zur interpersonellen Manipulation. Der zweite Faktor dagegen fokussiert auf eine Störung der Impulskontrolle, welches sich in einem erratischen und antisozialen Lebensstil äußert. ${ }^{825}$ Die empirische Untersuchung der Faktoren zeigt dabei weiter, dass die „klassischen“, mit Gewalttätigkeit assoziierten Psychopathen eine starke Ausprägung des ersten und zweiten Faktors aufweisen. Gewalttätige, allerdings nicht-psychopathische Individuen weisen nur einen erhöhten zweiten Faktor der verminderten Impulskontrolle auf. Besonders interessant erscheinen für die wirtschaftswissenschaftliche Forschung dagegen diejenigen Individuen, welche eine starke Ausprägung des ersten Faktors der Psychopathie aufweisen, jedoch eine relativ geringe Ausprägung des zweiten Faktors, woraus die Bezeichnung als Faktor 1-Psychopath resultiert. ${ }^{826}$ Die erörterten Zusammenhänge der zwei Faktoren zeigt auch nochmals die nachfolgende Abbildung 4.24.

Diese Individuen, welche eine starke Ausprägung des ersten, nicht aber des zweiten Faktors aufweisen, sind zwar nicht körperlich gewalttätig, weisen jedoch eine stark kaltherzig-furchtlose und opportunistisch-manipulative Tendenz auf, wodurch diese - gerade auch durch ihre Redegewandtheit und Extrovertiertheit oftmals fälschlich als starke Führungspersönlichkeiten, die auch in schwierigen Situationen harte Entscheidungen treffen können, eingeschätzt werden ${ }^{827}$ und

${ }^{824}$ STOUT bezeichnet diese Individuen daher auch treffend als ,ice people“ (Stout, M. (2005), S. 72).

825 Vgl. Hare, R. D. (1998), S. 101-102; Hare, R. D. (1996), S. 30; Babiak, P. (1995), S. 174; Hare, R. D. et al. (1990), S. 338-339; Harpur, T. J. / Hare, R. D. / Hakstian, A. R. (1989), S. 6-7; Harpur, T. J. / Hakstian, A. R. / Hare, R. D. (1988), S. 745. Frühe Überlegungen zu einer Zweifachdifferenzierung des Psychopathiekonstruktes finden sich schon bei Karpman, B. (1941). Vgl. auch Lehmann, R. J. B. et al. (2019), S. 1; Vaughn, M. G. et al. (2009), S. 173; Skeem, J. L. et al. (2007), S. 395.

${ }^{826}$ Vgl. Babiak, P. (2016), S. 357-358; Babiak, P. (2007), S. 415. Dabei kann festgestellt werden, dass gerade der Faktor 1 ,,personality traits that many clinicians consider to be at the core of psychopathy, including habitual lying, lovelessness, and guiltlessness" (Harpur, T. J. / Hakstian, A. R. / Hare, R. D. (1988), S. 745, vgl. auch Harpur, T. J. / Hare, R. D. / Hakstian, A. R. (1989), S. 6) umfasst. In diesem Sinne kann auch mit SMITH und LILIENFELD konstatiert werden: „Psychopathic personality, or psychopathy, is a constellation of personality traits and associated behaviors characterized by superficial charm, dishonesty, egocentricity, manipulativeness, risk-taking, and a lack of empathy and guilt masked by apparent normalcy.“ (Smith, S. F. / Lilienfeld, S. O. (2013), S. 206). Zur kritischen Diskussion, inwiefern eine analoge Bezeichnung als „Faktor 2-Psychopath“ konzeptionell sinnvoll erscheint, vgl. auch Lehmann, R. J. B. et al. (2019), S. 7; Skeem, J. L. et al. (2011), S. 115.

827 Vgl. Babiak, P. (2007), S. 419; Boddy, C. R. P. (2006), S. 1462. In diesem Sinne konstatieren auch BABIAK, NEUMANN und HARE: „It is easy to mistake psychopathic traits for 


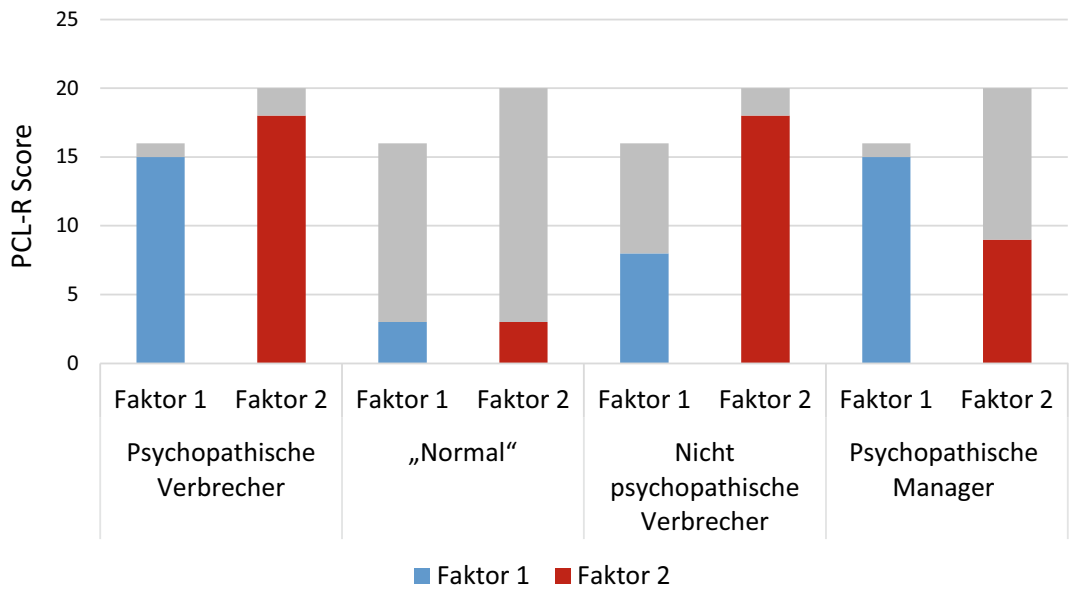

Abbildung 4.24 Arten psychopathischer Persönlichkeitsstörungen im Vergleich ihrer zweifaktoriellen Ausprägung der PCL-R ${ }^{828}$

dadurch in Unternehmen Karriere machen (,Psychopathen in Nadelstreifen“829). Allerdings ist für solche Individuen festzustellen, dass diese aufgrund ihres kaltherzigen, opportunistischen Charakters dazu neigen, ohne Gewissen oder Scham zu betrügen und zu lügen ${ }^{830}$, wodurch diese ein enormes Risikopotential sowohl auf organisationaler als letztlich auch auf gesellschaftlicher Ebene darstellen. Dies wird nochmals dadurch potenziert, dass durch den überproportionalen Aufstieg

specific leadership traits. For example, charm and grandiosity can be mistaken for selfconfidence or a charismatic leadership style; likewise, good presentation, communications, and impression management skills reinforce the same picture. [...] Even those traits that reflect a severe lack of human feelings or emotional poverty (lack of remorse, guilt, empathy) can be put into service by corporate psychopaths, where being ,tough ' or ,strong ' (making hard, unpopular decisions) or ,cool under fire" (not displaying emotions in the face of unpleasant circumstances) can work in their favor. In sum, the very skills that make the psychopath so unpleasant (and sometimes abusive) in society can facilitate a career in business even in the face of negative performance ratings." (Babiak, P. / Neumann, C. S. / Hare, R. D. (2010), S. 190-191).

${ }^{828}$ Beispielhafte Darstellung in Anlehnung an Babiak, P. (2016), S. 358. Die grauen Balken visualisieren jeweils die Maximalausprägung des spezifischen Faktors.

${ }^{829}$ So die treffende Bezeichnung bei Kühn, C. (2012).

${ }^{830}$ Vgl. Boddy, C. R. P. (2006), S. 1462. 
dieser Individuen ein empirisch nachweisbarer Aggregationseffekt psychopathischer Individuen auf höheren Managementebenen aufzufinden ist ${ }^{831}$, wodurch diese einerseits internen Kontrollmechanismen leichter entgehen können, andererseits aber auch durch ihre Position und Einflussmöglichkeiten eine integre Unternehmenskultur leichter über die Zeit zu korrumpieren vermögen.

Dabei bringt die aktuelle empirische Forschung Corporate Psychopaths mit einer Vielzahl organisationaler Probleme in Verbindung, wie beispielsweise einem „organisational decline in terms of revenue, employee commitment, creativity and organisational innovativeness. “832 Dieser organisationale Niedergang, welche Autoren wie BoDDY zur Bezeichnung psychopathischer Individuen als „organizational destroyers" ${ }^{* 33}$ führte, beruht auf mehreren Facetten. So betreiben Psychopathen häufig lediglich ,,impression management ${ }^{\star 834}$, sind also für die ihnen zugewiesene Stelle oft nicht oder nur unzureichend qualifiziert. ${ }^{835}$ In diesem Sinne konstatieren auch BABIAK, NEUMANN und HARE „that psychopathy

${ }^{831}$ Die Prävalenz in der Gesamtbevölkerung beträgt ca. $1 \%$ und nimmt statistisch mit steigendem Managementlevel zu. So finden einige Studien in oberen Managementebenen bis zu 3,5 \% (vgl. Boddy, C. R. P. / Ladyshewsky, R. K. / Galvin, P. (2010b), S. 127), 6 \% (vgl. Babiak, P. / Neumann, C. S. / Hare, R. D. (2010), S. 186) oder, in einer Studie im Bereich des Supply-Chain-Managements, sogar bis zu $21 \%$ (vgl. Fritzon, K. et al. (2017), S. 147) Individuen, welche einen so hohen Score erreichen, dass diese als Psychopathen zu klassifizieren sind. Vgl. auch Boddy, C. R. P. (2011a), S. 256; Boddy, C. R. P. (2005), S. 34; Hare, R. D. (1996), S. 26. Insgesamt kann so auch mit SMITH und LILIENFELD geschlussfolgert werden, dass genügend Hinweise „for an elevated prevalence of psychopathic personality traits in senior business management settings" (Smith, S. F. / Lilienfeld, S. O. (2013), S. 201) existieren.

832 Boddy, C. R. P. (2017), S. 141.

${ }^{833}$ So auch der Titel bei Boddy, C. R. P. (2011b). Vgl. ebenfalls Boddy, C. R. P. et al. (2015), S. 541-542. In diesem Sinne stellt BoDDY auch an anderer Stelle fest: „Although they may look smooth, charming, sophisticated, and successful, Corporate Psychopaths should theoretically be almost wholly destructive to the organizations that they work for." (Boddy, C. R. P. (2011a), S. 256). Ebenso konstatieren auch SMITH und LILIENFELD: „With few exceptions, these are individuals who are purportedly destructive to subordinates and companies alike." (Smith, S. F. / Lilienfeld, S. O. (2013), S. 206).

${ }^{834}$ Vgl. Boddy, C. R. P. (2017), S. 142; Perri, F. S. (2013), S. 341. So bspw. auch das bekannte Beispiel des ursprünglich als Postboten tätigen GERT POSTEL, welcher durch Redegewandtheit und Urkundenfälschung zeitweilig bis zum Oberarzt aufstieg. Vgl. Maercker, A. (2013), S. 42.

835 In diesem Sinne stellen auch BABIAK, NEUMANN und HARE fest: ,That is, high psychopathy total scores were associated with perceptions of good communication skills, strategic thinking, and creative/innovative ability and, at the same time, with poor management style, failure to act as a team player, and poor performance appraisals (as rated by their immediate bosses).“ (Babiak, P. / Neumann, C. S. / Hare, R. D. (2010), S. 189). 
is more strongly associated with style than with substance." 836 Ähnlich stellen BODDY und TAPLIN fest: „Psychopathic leadership is characterized by lying, such as claiming qualifications that the corporate psychopath does not actually possess. “837 Darüber hinaus ist durch die Anwesenheit eines Psychopathen mit einer signifikanten Verschlechterung des Arbeitsklimas zu rechnen, bspw. durch Mobbing oder Intrigen. So resümieren BODDY und TAPLIN ,that for most normal people, working under a corporate psychopath would usually entail the total destruction of job satisfaction. “ ${ }^{338}$ Dies führt einerseits zu einer Zerstörung des Commitments: „Therefore, it is not just physical withdrawal that is influenced by the presence of corporate psychopaths but also emotional withdrawal. “839 Andererseits führt die Anwesenheit eines psychopathischen Vorgesetzten neben steigender Personalfluktuation ${ }^{840}$ regelmäßig zu erhöhten Krankenständen und Fehlzeiten ${ }^{841}$ - teilweise wird sogar von einer chronischen Traumatisierung einiger unterstellter Mitarbeiter berichtet, welche noch nach Jahren Angststörungen aufweisen. ${ }^{842}$ Darüber hinaus führt das kontinuierliche Vorleben fragwürdigen Verhaltens durch Vorgesetzte (,walking bad behaviour") zu einer schleichenden moralischen Korruption der Organisation, insbesondere da Untergebene dazu neigen, das Verhalten ihrer Vorgesetzten zu imitieren, bzw. deren Werte in organisationalen Entscheidungsprozessen zu übernehmen. In diesem Sinne stellen auch BODDY, LADYSHEWSKY und GALVIN fest: „Furthermore, the mimicking and mirroring of organizational leaders' behaviour has been found in research into the toxic leadership of organizations [..] and this means that the presence of Corporate Psychopaths within an organization may well have an insidious effect on the ethical decision making of the whole organization [...]. ${ }^{\text {(843 }}$ So zeigen auch weitere

\footnotetext{
${ }^{836}$ Babiak, P. / Neumann, C. S. / Hare, R. D. (2010), S. 192.

${ }^{837}$ Boddy, C. R. P. / Taplin, R. (2016), S. 968.

838 Boddy, C. R. P. / Taplin, R. (2016), S. 975. Vgl. auch Mathieu, C. / Babiak, P. (2016), S. 104-105; Boddy, C. R. P. (2014), S. 111-115; Mathieu, C. et al. (2014), S. 85-86; Boddy, C. R. P. (2011b), S. 96-98.

${ }^{839}$ Boddy, C. R. P. et al. (2015), S. 537. Vgl. auch Boddy, C. R. P. (2017), S. 147.

${ }^{840}$ Vgl. Boddy, C. R. P. et al. (2015), S. 537.

${ }^{841}$ Vgl. Oyewunmi, A. E. / Akinnusi, D. M. / Oyewunmi, O. A. (2018), S. 151-154; Boddy, C. R. P. (2017), S. 150.

${ }^{842}$ Vgl. Boddy, C. R. P. / Taplin, R. (2016), S. 975. Dies führte ClARKE zur Bezeichnung als „Working with Monsters“ (Clarke, J. (2005)). So kann auch mit BoDDY konstatiert werden: „Corporations that wish to maximize the well-being of their employees and to minimize conflict and bullying will have to minimize their employment of Corporate Psychopaths [...].“ (Boddy, C. R. P. (2014), S. 118).

${ }^{843}$ Boddy, C. R. P. / Ladyshewsky, R. K. / Galvin, P. (2010b), S. 125.
} 
Studien den negativen Einfluss psychopathischer Führungskräfte auf unternehmerische Nachhaltigkeitsentscheidungen. ${ }^{844}$ Des Weiteren betreiben Psychopathen, wie bereits im Kontext des Homo oeconomicus verdeutlicht, regelmäßig eine harte opportunistische Eigennutzverfolgung, welche häufig von der Wertschöpfung der Untergebenen profitiert, ohne selbst hierzu wesentlich beizutragen. Dies resümiert auch BODDY im Rahmen einer Case Study wie folgt: ,[C]orporate psychopaths are reported to be parasitic in that they feed off the good work of others and this current research confirms this viewpoint. The psychopathic CEO has reportedly strengthened his own position and external reputation while weakening the organisation that employs him, especially in terms of its human resource capability and overall performance." ${ }^{\circ 45}$

Schließlich konnte neuerdings auch erstmalig gezeigt werden, dass Psychopathen, insbesondere in den Ausprägungen des traditionellen Homo oeconomicus, d. h. Kaltherzigkeit und opportunistischem Eigennutzstreben ${ }^{846}$, wirtschaftskriminellen Handlungen eher zustimmen. ${ }^{847}$ Gekoppelt mit fehlender Reue und Furchtlosigkeit $^{848}$ sowie der Unfähigkeit aus Sanktionen oder Strafandrohungen zu lernen ${ }^{849}$, stellen Psychopathen folglich einen großen organisationalen Risikofaktor dar, wie der Untergang einiger Großunternehmen (so z. B. auch Enron ${ }^{850}$ ) verdeutlicht. In diesem Sinne stellt auch BODDY fest, dass ,once great companies of longstanding history and with previously unblemished and even dignified reputations have been brought down by the misdeeds of a few of their leaders. “ 851

${ }^{844}$ Vgl. Myung, J. K. / Choi, Y. H. / Kim, J. D. (2017), S. 9-15; Boddy, C. R. P. / Ladyshewsky, R. K. / Galvin, P. (2010a), S. 6-11. Vgl. im Kontext ethischer Entscheidungen ähnlich auch Stevens, G. W. / Deuling, J. K. / Armenakis, A. A. (2012), S. 144-146.

845 Boddy, C. R. P. (2017), S. 152. Vgl. auch Boddy, C. R. P. et al. (2015), S. 545.

${ }^{846}$ So sind auch nach BODDY Psychopathen im Unternehemenskontext ,those personalities who are highly likely to act for themselves rather than as guardians or custodians of the organizations they manage." (Boddy, C. R. P. (2016b), S. 86).

${ }^{847}$ Vgl. Lingnau, V. / Fuchs, F. / Dehne-Niemann, T. E. (2017), S. 1208-1212.

848 Dies stellen auch BODDY, LADYSHEWSKY und GALVIN wie folgt fest: „Corporate Psychopaths are reported to have a great need for stimulation and this leads them to take financial, legal, or moral risks that others would not take [...]." (Boddy, C. R. P. / Ladyshewsky, R. K. / Galvin, P. (2010a), S. 3).

${ }^{849}$ Vgl. Godman, M. (2018), S. 231-232; Viding, E. / McCrory, E. J. (2012), S. 969; Blair, K. S. et al. (2006), S. 156; Blackburn, R. (1983), S. 187.

${ }^{850} \mathrm{Vgl}$. hierzu sowie zu anderen Fälle psychopathischer Führungskräfte und deren negativen Implikationen im unternehmerischen Kontext Lingnau, V. / Fuchs, F. / Dehne-Niemann, T. E. (2017), S. 1194; Boddy, C. R. P. (2016a), S. 262; Boddy, C. R. P. et al. (2015), S. 531. ${ }^{851}$ Boddy, C. R. P. (2011a), S. 255. Vgl. auch Boddy, C. R. P. / Ladyshewsky, R. K. / Galvin, P. (2010b), S. 121. 
Ähnlich schlussfolgern auch BoDDY ET AL.: ,[I]t has been theorized that their presence and influence will ultimately lead to organizational destruction and that an ethically bankrupt organization will become financially bankrupt [...]. 852 Hierdurch kommt es schließlich dazu, dass Psychopathen ,destroy[] the company from within by causing good people to leave, needlessly abandoning good business plans and by destroying its ethical reputation." ${ }^{853}$

Darüber hinaus sind allerdings auch einige gesellschaftliche Konsequenzen zu nennen. So werden die betrügerischen Entscheidungen psychopathischer Führungskräfte für wirtschaftliche Schäden in Milliardenhöhe sowie wesentlich für die Finanzkrise mitverantwortlich gemacht ${ }^{854}$ - mit der Konsequenz einer wiederum global notwendigen staatlichen Intervention zur Sicherung des Bankenwesens und der Finanzmärkte, welche wiederum weltweit zu einer stark ansteigenden Staatsverschuldung führte. Des Weiteren haben die historischen Unternehmensschieflagen evident nicht nur Auswirkungen auf die Shareholder, sondern die Gesamtheit der Stakeholder - so auch bspw. die Mitarbeiter der Unternehmen sowie deren Familien. In diesem Sinne resümiert BoDDY: „This is important because when large financial corporations are destroyed by the actions of their senior directors, employees loose [sic!] their jobs and sometimes their livelihoods, shareholders lose their investments and sometimes their life savings and societies lose key parts of their economic infrastructure. Capitalism also loses some of its credibility. “855 Gerade letzterer Aspekt weist darauf hin, dass die vielfältig vorkommenden moralischen Fehltritte nicht nur zu einer Untergrabung der einzelnen unternehmerischen Legitimität führen, sondern ebenso auf der Makroebene das Vertrauen in das marktwirtschaftliche System als Ganzes bedrohen. So stellen auch etliche Autoren fest, dass sich das Vertrauen in das Wirtschaftssystem auf einem historisch niedrigen Stand befindet. ${ }^{856}$ In der Gesamtschau

852 Boddy, C. R. P. et al. (2015), S. 541-542.

${ }^{853}$ Boddy, C. R. P. et al. (2015), S. 542.

${ }^{854}$ Vgl. Hossiep, R. / Ringelband, O. (2014), S. 24; Marshall, A. J. / Baden, D. / Guidi, M. (2013), S. 559; Boddy, C. R. P. (2011a), S. 255-256.

855 Boddy, C. R. P. (2011a), S. 255. Ebenso konstatiert auch ALLIO, dass ,leaders who are toxic $[\ldots]$ damage the interests and welfare of the stakeholders. They inflict pain and suffering on their organizations, and on the individuals who follow them, including employees and shareholders.“ (Allio, R. J. (2007), S. 12). HARE bezeichnet diese Individuen auch als ,social predators who charm, manipulate, and ruthlessly plow their way through life, leaving a broad trail of broken hearts, shattered expectations, and empty wallets." (Hare, R. D. (1999), S. XI).

${ }^{856}$ Vgl. Horváth, D. / Barton, D. (2016), S. 319; Pies, I. / Hielscher, S. / Beckmann, M. (2009a), S. 324. 
kann damit in Bezug auf die organisationalen sowie gesellschaftlichen Konsequenzen mit BODDY konstatiert werden, dass Psychopathen „therefore a menace to the companies they work for and to society“857 sind. Ähnlich betonen auch HoSSIEP und RINGELBAND: ,[S]ie können ganze Unternehmen oder gar Volkswirtschaften zugrunde richten." ${ }^{\circ 58}$ Ebenso resümieren schließlich auch WALKER und JACKSON: „Moral emotion deficits potentially confer advantages on individuals to reap themselves business rewards such as money and status. However, these same rewards are unlikely to confer an advantage on the organization or the broader society $[\ldots]$... 859

Damit ergibt sich aus wirtschaftsethischer Perspektive die Frage, welche Möglichkeiten der Prävention gegenüber Psychopathen bestehen. ${ }^{860}$ Bisher vorgeschlagene Ansätze wie Screeningprozeduren bei der Rekrutierung von Mitarbeitern $^{861}$ erscheinen allerdings nicht zielführend, denn diese können durch Psychopathen als „Meister der Täuschung“ leicht umgangen werden. ${ }^{862}$ Aber auch laufende Screenings, so z. B. mit dem von MATHIEU ET AL. entwickelten BusinessScan360 863 , sowie einer reinen Sensibilisierung des Personals für die Thematik ${ }^{864}$ führt möglicherweise zu einer Kultur des Misstrauens sowie der Gefahr, dass psychopathische Individuen Kollegen durch geschickte Fehlinformationen gegeneinander aufwiegeln. Darüber hinaus hat die Literatur auch die Nutzung von „Red Flags“ (z. B. zunehmende Krankenstände, Fehlzeiten

${ }^{857}$ Boddy, C. R. P. (2011a), S. 256.

${ }^{858}$ Hossiep, R. / Ringelband, O. (2014), S. 24.

${ }^{859}$ Walker, B. R. / Jackson, C. J. (2017), S. 805.

${ }^{860}$ BoDDY ruft in diesem Zusammenhang sogar pointiert zu einem „,call to arms“ (Boddy, C. R. P. (2005), S. 30) auf.

${ }^{861}$ Vgl. Walker, B. R. / Jackson, C. J. (2017), S. 807; Boddy, C. R. P. (2016a), S. 254; Boddy, C. R. P. (2016b), S. 86-87; Boddy, C. R. P. (2014), S. 118; Mathieu, C. et al. (2013), S. 288289. Auch PERRI stellt in diesem Sinne fest, dass ,,some have suggested that CEOs and other corporate executives should be screened for psychopathic traits, given the billions of dollars they are asked to manage.“ (Perri, F. S. (2013), S. 339).

${ }^{862}$ Vgl. Marshall, A. J. et al. (2015), S. 499; Hossiep, R. / Ringelband, O. (2014), S. 24; Pech, R. J. / Slade, B. W. (2007), S. 264. Dies gilt sogar für eine Beurteilung durch erfahrene Psychologen, wie etwa MATHIEU ET AL. hervorheben: „Even trained industrial/organizational (I/O) psychologists, if not made aware of problematic evaluations or profiles, may think certain individuals are good fits for the organization when, in fact, they are potentially toxic." (Mathieu, C. et al. (2013), S. 292). Vgl. auch Hare, R. D. (1999), S. 35.

${ }^{863}$ Vgl. grundlegend Mathieu, C. et al. (2013).

${ }^{864}$ Vgl. Boddy, C. R. P. (2006), S. 1470. 
etc.) als Warnindikatoren vorgeschlagen. ${ }^{865}$ Obschon komplementär überaus sinnvoll, wirken diese ebenfalls lediglich ex post, können also den Eintritt in die Organisation (ex ante) kaum verhindern. Es gilt daher, wie auch IRTELLI und VINCENTI feststellen, die ,importance of primary prevention“866. Hierzu wurden neben Befragungen teilweise auch medizinische Tests, wie bspw. ein Gehirnscan mittels Magnetresonanztomographie [MRT], vorgeschlagen. So weisen Psychopathen eine deutlich geringere Erregung in bestimmten, für die Verarbeitung emotionaler Stimuli notwendigen Gehirnarealen auf, wenn diese Individuen z. B. mit Abbildungen von Gewalt oder Leid konfrontiert werden, welches wiederum mittels MRT sichtbar gemacht werden kann. ${ }^{867}$ Allerdings zeigen sich hier neben praktischen Problemen der Durchführung auch etliche rechtliche wie ethische Fragen der Zulässigkeit solcher Prozeduren bei der Personalakquise. Aus diesem Grunde erscheint für die weitere Forschung insbesondere die Erforschung psychopathieresistenter Strukturen notwendig, so dass eine Organisation bereits zu Beginn als nicht attraktiv für psychopathische Individuen erscheint. ${ }^{868}$ Neben der Veränderung der organisationalen Abläufe, wie teilweise vorgeschlagen wurde ${ }^{869}$, scheint gerade die Erforschung der gegenwärtig vorherrschenden Entlohnungssysteme fruchtbar zur Gestaltung psychopathieresistenter Organisationen. Damit ergibt sich auch ein expliziter Anknüpfungspunkt für die Controllingforschung wie -praxis. Folgt man der oben skizzierten Argumentation KUHNS, so sollten gerade die traditionellen, individualorientierten monetären Incentives (,Bonuszahlungen“), welche nach wie vor in vielen Großunternehmen

${ }^{865}$ Vgl. Babiak, P. / Hare, R. D. (2019), S. 254-262; Boddy, C. R. P. / Taplin, R. (2016), S. 973-977; Lingnau, V. / Dehne-Niemann, T. E. (2016), S. 34-35.

${ }^{866}$ Irtelli, F. / Vincenti, E. (2017), S. 193.

${ }^{867}$ Vgl. Moreira, D. / Azeredo, A. / Barbosa, F. (2019), S. 139-156; Poeppl, T. B. et al. (2019), S. 464-467; Fallon, J. (2013), S. 11 und S. 58-63; Anderson, N. E. / Kiehl, K. A. (2012), S. 54-58; Sato, J. R. et al. (2011), S. 632-637; Gao, Y. et al. (2009), S. 814-815; Boddy, C. R. P. (2006), S. 1465.

${ }^{868}$ Vgl. Lingnau, V. / Fuchs, F. / Dehne-Niemann, T. E. (2017), S. 1216; Kühn, C. (2012), S. $188-190$.

${ }^{869}$ So z. B. bei SHERMAN, welcher eine Abschreckung durch erhöhte Bürokratiemaßnahmen vorschlägt, so dass ein solches Unternehmen ,neither attractive nor vulnerable to the psychopath“ (Sherman, C. (2000), S. 38) wäre. Ebenso stellt auch DEUTSCHMAN fest: „A slow-moving old-economy corporation would be too boring for a psychopath, who needs constant stimulation." (Deutschman, A. (2005)). Betrachtet man allerdings die Notwendigkeit in einem globalisierten Wettbewerb eher Bürokratie abzubauen, so erscheint, zumindest alleinig auf der Mesoebene (ohne Änderung der Weltwirtschaftsordnung, d. h. der globalen Makroebene), ein solcher Ansatz schwierig praktisch erfolgreich umzusetzen. 
vorherrschend sind, Individuen mit diesen problematischen Charakterzügen anziehen. Im Umkehrschluss sollten soziale Arbeitsbedingungen mit festen Löhnen eher abschreckend auf psychopathische Individuen wirken. Diese Überlegungen wurden schließlich in einer aktuell noch unveröffentlichten Studie des Autors dieser Arbeit anhand zweier Leithypothesen ausformuliert und nochmals anhand des gewählten Erhebungsinstruments der PPI-R ${ }^{870}$ genauer spezifiziert. ${ }^{871}$ Hierbei wurden insgesamt 210 Professionals via Social Networks sowie persönlicher Kontakte akquiriert, welche sich wiederum aus 118 Männern sowie 92 Frauen zusammensetzen (inter/divers/sonstige: 0). Außerdem sind 54 Personen dem oberen Management zuzuordnen, davon 20 CFOs großer deutscher Unternehmen des MDAX. Die inferenzstatistische Analyse bestätigt im Kern die aufgezeigten Hypothesen. Es kann festgestellt werden, dass mit zunehmendem Grad der Psychopathie Individuen ein Unternehmen mit traditionellen monetären Einzelincentives attraktiver bewerten. Umgekehrt werden Unternehmen, welche sehr stark auf Teamarbeit und Gruppenboni setzen, mit steigendem Aus$\mathrm{ma} ß$ an Psychopathie schlechter evaluiert. Darüber hinaus werden insbesondere aber auch bei feinerer Auflösung der PPI-Skala die vorigen Hypothesen bestätigt. So zeigt sich, dass die Kerneigenschaften des Faktor 1-Psychopathen im Sinne des Homo oeconomicus-Paradigmas, d. h. insbesondere Kaltherzigkeit sowie Machiavellistischer Egoismus, eine zentrale Rolle bei der Attraktivitätsbewertung von Incentiveschemata einnehmen. Kurz subsumiert kann festgestellt werden, dass im Rahmen klassischer Anreizsysteme insbesondere die Ausprägung des Machiavellistischen Egoismus einen hochsignifikanten positiven Einfluss auf die Attraktivitätsbewertung hat, während dieser Faktor hochsignifikant negativ im Bereich der sozialen Arbeitsbedingungen bzw. Entlohnung (Gruppenbonus) ausfällt, darüber hinaus ist auch der Faktor Kaltherzigkeit signifikant negativ in Bezug auf die Attraktivitätsevaluation der sozialen bzw. teamorientierten Entlohnung. Diese Studie bestätigt folglich die Vermutung, dass gerade traditionelle Incentives bzw. klassische Anreizsysteme, wie diese nach wie vor in

${ }^{870}$ Die PPI-R eignet sich als Selbstbewertungsinstrument der Psychopathie besonders zur Erhebung im nichtklinischen (Online-)Surveykontext. Sie setzt sich aus insgesamt acht Subskalen (Schuld-Externalisierung, Rebellische Risikofreude, Stressimmunitat, Sozialer Einfluss, Kaltherzigkeit, Machiavellistischer Egoismus, Sorglose Planlosigkeit und Furchtlosigkeit) zusammen. Vgl. grundlegend Lilienfeld, S. O. / Widows, M. R. (2005) sowie die Anmerkungen in Fn. 820. In der Studie kam dabei eine verkürzte und validierte Version (PPIR-40) der deutschen Übersetzung von Alpers, G. W. / Eisenbarth, H. (2008) nach Eisenbarth, H. / Lilienfeld, S. O. / Yarkoni, T. (2015) zum Einsatz.

${ }^{871}$ Vgl. hierzu im Folgenden Lingnau, V. / Fuchs, F. / Dehne-Niemann, T. E. (2021). 
vielen großen Industrieunternehmen, aber gerade auch im Finanzwesen dominieren, in der Tat psychopathische Persönlichkeiten (bzw. insbesondere Faktor 1-Psychopathen bzw. „Corporate Psychopaths“) anziehen, während eine sozialere Entlohnungsstruktur abschreckend wirkt. Die Abkehr von traditionellen Individualboni und die Ermöglichung von mehr sozialer Interaktion und teambasierter Evaluation könnte demnach zukünftig ein bedeutendes Element einer psychopathieresistenteren Organisation darstellen. Beachtet man das zuvor aufgezeigte Risiko bzw. überaus destruktive Potential, welches psychopathisches Führungspersonal für den dauerhaften unternehmerischen Erfolg darstellt, so gilt vor dem aufgezeigten Hintergrund für das Controlling, dass dieses das Management bei Entscheidungen über den Einsatz von traditionellen Anreizsystemen klar über dessen Grenzen und insbesondere auch über die Gefahren einer Anziehung problematischer Charakterzüge im Sinne des negativen Selektionseffekts informieren muss.

Allerdings ist auch festzuhalten, dass ein verantwortungsvolles unternehmerisches Handeln noch nicht unkritisch bzw. alleinig durch extrinsische Anreize oder Sanktionen motiviert werden kann, denn dies würde aus mehreren Gründen zu kurz greifen, weshalb dem Begriff der Organisations- bzw. Unternehmenskultur $^{872}$ eine zentrale Bedeutung zukommt. Hiermit befasst sich die Arbeit im nachfolgenden Kapitel. ${ }^{873}$

\subsection{Verankerung unternehmensethischer Maßnahmen innerhalb einer integren Unternehmenskultur}

Der Begriff der Unternehmens- bzw. Organisationskultur stellt einen Transfer des ursprünglich kulturanthropologischen Begriffs auf die Mesoebene dar. Obschon viele Autoren die große inhaltliche Bandbreite des allgemeinen Kulturbegriffs hervorheben ${ }^{874}$, haben einige Autoren Definitionen dieses Begriffs

\footnotetext{
${ }^{872}$ Wie im Zusammenhang der Ziele schon hervorgehoben, handelt es sich im strengen Sinne hier begrifflich eigentlich um die Organisationskultur bzw. die Kultur des sozialen Systems des Unternehmens. Im Weiteren sei der Begriff der Unternehmenskultur als verbreiteter ,terminus technicus" jedoch relativ synonym hierzu gebraucht. Vgl. in diesem Kontext auch Fabisch, N. / Elle, M. (2012), S. 213.

${ }^{873}$ Das Konstrukt der Kultur steht, wie zuvor erörtert wurde, auch im Fokus der kulturalistischen Wirtschafts- und Unternehmensethik. Vgl. hierzu nochmals Abschnitt 3.4.5.2.

${ }^{874}$ Vgl. z. B. Eagleton, T. (2016), S. 1-3; Hoppe, B. M. / Heinze, T. (2016), S. 6; Bendixen, P. (2011), S. 129; Nassehi, A. (2010), S. 375; Eagleton, T. (2000), S. 1. In diesem Zusammenhang stellt auch LUDDEMANN paradigmatisch fest: „,Die Klage über die Unschärfe
} 
vorgeschlagen. ${ }^{875}$ So stellt etwa LUDDEMANN fest: „Kultur ist ein Gefüge aus Bedeutungskomplexen, das Sinnangebote bereitstellt." ${ }^{876}$ Darüber hinaus konstatiert NASSEHI: „Kultur versorgt uns [.] mit Chiffren darüber, was wie und für wen bedeutsam ist." ${ }^{877}$ Eine vielzitierte Definition stammt auch von KLUCKHOHN, welcher Kultur definierte als ,patterned ways of thinking, feeling, and reacting, acquired and transmitted mainly by symbols, constituting the distinctive achievements in human groups, including their embodiments in artifacts; the essential core of culture consists of traditional (i.e., historically derived and selected) ideas and especially their attached values. ${ }^{* 878}$ Im Kern des Kulturbegriffs geht es folglich um die Vermittlung von Sinn sowie bestimmten Werten, welche, wie klassisch HOFSTEDE feststellte, als ,collective programming of the mind“879 bzw. nach GEERTZ als „webs of significance“ ${ }^{\text {“80 }}$ fungieren. Die sozialwissenschaftliche Forschung hat dabei in den letzten Jahrzehnten den ursprünglich gesellschaftswissenschaftlichen Begriff auf die Mesoebene übertragen und somit der organisationswissenschaftlichen Forschung zugänglich gemacht, denn auch hier nehmen gemeinsam geteilte Wertvorstellungen unmittelbaren Einfluss auf die unternehmerische Praxis. ${ }^{881}$

Die Bedeutung von Kultur lässt sich vor dem Hintergrund der vorigen Ausführungen an mehreren Punkten festmachen. So ist zuerst einmal festzustellen, dass

des Begriffes der Kultur [...] gehört ebenso zur Beschäftigung mit seinem Gegenstand wie dessen überwältigende Allgegenwart.“ (Lüddemann, S. (2019), S. V).

${ }^{875}$ Zur Übersicht über weitere Definitionsvorschläge vgl. auch Senior, B. / Swailes, S. / Carnall, C. (2020), S. 133-134; Clark, K. E. / Clark, M. B. (1999), S. 204-206; Erez, M. / Earley, P. C. (1993), S. 41-42.

${ }^{876}$ Lüddemann, S. (2019), S. 5. Zur historischen Entwicklung des Begriffs aus dem lateinischen cultura bzw. cultus vgl. auch Lüddemann, S. (2019), S. 1; Hoppe, B. M. / Heinze, T. (2016), S. 4-6; Klein, G. (2016b), S. 278; Moebius, S. (2010), S. 14-15; Ort, C.-M. (2008), S. 19-20.

${ }^{877}$ Nassehi, A. (2011), S. 289; Nassehi, A. (2010), S. 375.

${ }^{878}$ Kluckhohn, C. (1951), S. 86, Fn. 5 Kapitel 1.

${ }^{879}$ Hofstede, G. (2001), S. 1. Nach HoFSTEDE können darüber hinaus insgesamt fünf Dimensionen der Kultur unterschieden werden: 1. Die Machtdistanz, 2. der Grad der Unsicherheitsvermeidung, 3. Individualismus vs. Kollektivismus, 4. Maskulinität vs. Feminität sowie 5. Kurz- vs. Langfristorientierung (vgl. Hofstede, G. (2001), S. 29).

${ }^{880}$ Geertz, C. (1973), S. 5.

${ }^{881}$ So auch in dem bekannten Mehrebenenmodell der Organisationskultur nach Schein, E. H. / Schein, P. (2017), S. 17-25. SCHEIN und SCHEIN definieren dabei Kultur grundlegend als ,a pattern or system of beliefs, values, and behavioral norms that come to be taken for granted as basic assumptions and eventually drop out of awareness." (Schein, E. H. / Schein, P. (2017), S. 6). 
extrinsische Anreizsysteme zur (angestrebten) Kultur des Unternehmens passen müssen, da diese neben dem Hervorrufen von Problemen in der wirksamen Führung sonst auch intendierte kulturelle Veränderungen konterkarieren können. ${ }^{882}$ Darüber hinaus ist bei alleinigem oder zu starkem Fokus auf die Mitarbeiterführung durch extrinsische Incentives die Gefahr eines moralischen Crowding Outs zu nennen ${ }^{883}$, d. h. die Verdrängung einer verinnerlichten Moralbeachtung (Ethos) bzw. ein Handeln aus Überzeugung durch extrinsische Anreize. Hinsichtlich einer auch an intrinsischer Motivation orientierten Führung der Mitarbeiter nimmt eine reflektierte Betrachtung der Unternehmenskultur folglich eine zentrale Position ein. Schließlich, und dies ist ebenfalls von Bedeutung, existiert das Problem der wirkungsvollen praktischen Implementierung von unternehmensethischen Maßnahmen (z. B. im Rahmen des Analytischen Frameworks). So ist gerade eine Unternehmenskultur, welche für ethische Überlegungen hinreichend anschlussfähig ist, für die Erfolgsaussichten einer Implementation unternehmensethischer bzw. normativer Instrumente bedeutsam. In diesem Sinne konstatiert auch DOBIASCH, ,dass die Organisationskultur tatsächlich eine zentrale Bedeutung für den Erfolg oder Misserfolg der Implementierung von Ethikmanagement hat. "884 Insbesondere scheint klar, dass die wirksame Integration bzw. Institutionalisierung von unternehmensethischen Erkenntnissen in einem normativen Management die Bereitschaft voraussetzt, Ethikprogramme nicht nur beiläufig als „Labels“ oder kurzfristig wirksame Kommunikationsprogramme zu erachten, sondern die Maßnahmen durch alle Organisationshierarchien ernsthaft zu verfolgen. So darf sich die Umsetzung, wie auch analog im Bereich der Legitimierungsansätze und den Grenzen eines rein symbolischen Managements bereits herausgestellt ${ }^{885}$, evident nicht nur lediglich proklamativ in der Unternehmenskommunikation (bspw. auf der Homepage bzw. im Geschäftsbericht) niederschlagen, sondern muss auch tatsächlich durch alle Ebenen des Unternehmens verfolgt werden, da die Maßnahmen sonst ins Leere laufen. Hierzu ist dann auch der „tone from the top“ entscheidend, d. h. gerade die obersten Managementebenen müssen kulturprägend und integer diese verantwortungsbewusste Kultur vorleben und ernsthaft unterstützen. Dabei wird gerade im ethischen

\footnotetext{
${ }^{882}$ Ein bekannter Fall stammt aus dem Jahre 2012 bei der Deutschen Bank, als der proklamierte „Kulturwandel“ mit den real nach wie vor existierenden individual- und kurzfristorientierten Anreizsystemen konfligierte. Vgl. Rexer, A. (2012).

${ }^{883} \mathrm{Vgl}$. hierzu nochmals die Überlegungen in Abschnitt 3.6.1.

${ }^{884}$ Dobiasch, S. (2014), S. 32. Vgl. hierzu auch Grabner-Kräuter, S. (2000), S. 303.

885 Vgl. hierzu nochmals Abschnitt 4.4.3.2.
} 
Forschungskontext häufig die große Bedeutung der Integrität der Unternehmenskultur als „Erfolgsfaktor[] guter Unternehmenspraxis und gesellschaftlicher Wohlfahrt" ${ }^{886}$ hervorgehoben.

Hinsichtlich der Bestimmung des Begriffs der Integrität existieren ebenfalls verschiedene konzeptionelle Vorschläge. ${ }^{887}$ So fassen etwa einige Autoren Integrität vorherrschend deskriptiv im Sinne einer Verlässlichkeit oder einer Orientierung an inneren Werten (Ethos) auf. In diesem Sinne konstatiert etwa RADTKE mit Mikrofokus: „Eine Person ist in dem Grade integer, in dem sie fortwährend ihr Handeln an ihren Werten und Grundsätzen ausrichtet. Einem Menschen schreibt man zu Recht Integrität zu, wenn er bei seinem Handeln konsequent seinen inneren Werten folgt und diese gegenüber äußeren positiven und negativen Sanktionen sowie anderen Konsequenzen bevorzugt. “888 Ebenso stellen GRASSL und SCHMUTZ fest: „Integrität ist die Übereinstimmung der persönlichen Werte mit dem eigenen Handeln." ${ }^{889}$ In diesem Sinne definieren auch YODER-WISE, KOWALSKI und SPORTSMAN: „Integrity is being honest, consistent, and uncompromising [...]. We demonstrate integrity when we act in alignment with our stated values, beliefs, and principles." ${ }^{890}$ Schließlich definiert WENDEL Integrität ,in the sense of maintaining fidelity over time to one's own commitments and loyalties." 891

Darüber hinaus existieren jedoch auch Ansätze, welche explizit auf einer normativen Basis aufbauen. So beginnen etwa HUGELMEYER und GLÖGGLER zwar scheinbar rein deskriptiv mit der Feststellung, dass es bei Integrität ,um die Übereinstimmung von Werten mit konkretem Reden und Handeln; sowohl eine Frage der Authentizität als auch der Bedeutung von Grundüberzeugungen“892 gehe, fügen jedoch schon wenig später konkretisierend hinzu: „Integres Führungshandeln schließt auch ein, die Integrität und Würde anderer Menschen

\footnotetext{
886 Wieland, J. (2005b), S. 13.

${ }^{887}$ Der Begriff stammt aus dem lateinischen integer bzw. integritas, d. h. ganz, makellos, rein oder unkorrumpiert zu sein. Vgl. Keller, T. (2019), S. 3; Schöttl, L. (2018), S. 41; Renz, P. S. / Frischherz, B. / Wettstein, I. (2015), S. 4; Kasulis, T. P. (2002), S. 25.

${ }^{888}$ Radtke, B. (2015), S. 54, welcher zudem die Relevanz einer inhaltlichen Ausrichtung der zugrunde liegenden Werte auf die Organisation betont.

${ }^{889}$ Grassl, J. / Schmutz, C. R. (2014), S. 43.

${ }^{890}$ Yoder-Wise, P. S. / Kowalski, K. / Sportsman, S. (2021), S. 116.

891 Wendel, W. B. (2010), S. 241.

${ }^{892}$ Hügelmeyer, P. / Glöggler, A. (2020), S. 33-34.
} 
zu respektieren." ${ }^{893}$ Noch deutlicher normativ versteht etwa ULRICH, konsistent zu seiner Wirtschaftsethikkonzeption, welche auf dem Konzept des zivilisierten Wirtschaftsbürgers aufbaut, Integrität wie folgt: „Integer denkt und handelt, wer auch im Wirtschaftsleben den Kriterien eines ,zivilisierten' Umgangs mit anderen treu bleibt." ${ }^{\text {894 }}$ An anderer Stelle konstatieren ebenfalls ULRICH und KAISER: „Eine konsequent (vor-)gelebte Integritäts- und Verantwortungskultur im Unternehmen soll Mitarbeitende auf allen Ebenen zu einem kritisch-loyalen Mitdenken befähigen, berechtigen und ermutigen." 895 Gleichsam normativ geprägt stellen auch DUNGY und WHITAKER fest: „Integrity is what you do when no one is watching: it's doing the right thing all the time, even if it may work to your disadvantage." ${ }^{896}$ Auch LENNICK, KIEL und JORDAN resümieren auf normativer Basis: „When we act with integrity, we harmonize our behavior to conform to universal human principles.“897 Schließlich konstatiert auch DE GEORGE pointiert: „Acting with integrity is the same as acting ethically or morally.“ 898

Das Konzept der Integrität wird dabei organisational wiederum häufig einem reinen Complianceprogramm gegenübergestellt. ${ }^{899}$ Dabei zeigt die Durchsicht der Literatur, dass interessanterweise beide Ansätze nicht nur, wie die Bezeichnung nahelegt, auf die zugrunde liegende Norm fokussieren. In diesem Zusammenhang scheint aus Sicht der vorausgegangenen Überlegungen klar, dass ein reines Compliancemanagement bereits inhaltlich als nicht hinreichend zur dauerhaften Sicherung der Unternehmensexistenz zu erachten ist. Darüber hinaus zeigt sich allerdings auch, dass beide Ansätze ebenfalls aus motivationaler Perspektive in Bezug auf die Organisationsmitglieder auf konträren Auffassungen basieren,

${ }^{893}$ Hügelmeyer, P. / Glöggler, A. (2020), S. 34, in der Quelle ebenfalls herv. Ähnlich stellt auch KELLER fest: ,Ein integrer Mensch handelt demnach in dem Bewusstsein, seine persönlichen Überzeugungen und Werte zu vertreten, sich selbst treu zu bleiben, andere Menschen in ihrer Integrität nicht zu verletzen und zudem noch die gesellschaftlichen Rahmenbedingungen zu berücksichtigen." (Keller, T. (2019), S. 3).

${ }^{894}$ Ulrich, P. (2011c), S. 51.

895 Ulrich, P. / Kaiser, M. (2001), S. 27.

${ }^{896}$ Dungy, T. / Whitaker, N. (2011), S. 12.

${ }^{897}$ Lennick, D. / Kiel, F. / Jordan, K. (2011), S. 21.

${ }^{898}$ De George, R. T. (1993), S. 5.

${ }^{899}$ Vgl. Schöttl, L. (2018), S. 98-105; Heißner, S. (2014), S. 171; Noll, B. (2013), S. 185187; Hesseler, M. (2011), S. 108; Kleinfeld, A. / Kettler, A. (2011), S. 275-279; Verhezen, P. (2010), S. 187; Clausen, A. (2009), S. 32-33; Kreikebaum, H. (2006), S. 10-11; Steinmann, H. / Löhr, A. (2002b), S. 112; Paine, L. S. (1994), S. 109-113. 
welches wiederum auf die zugrunde liegende Kultur referenziert. ${ }^{900}$ So ist bei den meisten klassischen Complianceprogrammen ein stark extrinsisch motivierter bzw. kontrollorientierter Charakter festzustellen. In diesem Fall liegt dann nicht nur ein starker Fokus auf der Konformität mit externen Standards, um kriminelles Fehlverhalten zu verhindern. Vielmehr wird darüber hinaus auch den Mitarbeitern, basierend auf den traditionellen informationsökonomischen Überlegungen, ein potentiell unbegrenzter Opportunismus unterstellt, welches zur (scheinbaren) Notwendigkeit des Aufbaus umfangreicher Überwachungssysteme und der Androhung von Sanktionsmaßnahmen zur Erzielung von Compliance führt. Demgegenüber basieren Ansätze der Literatur, welche auf ein Integritätsmanagement setzen, neben grundlegenden, absichernden Kontrollmaßnahmen ${ }^{901}$ wesentlich stärker auf einer Selbstkontrolle, Selbstverantwortung und der moralischen Vernunftfähigkeit sozialer Wesen. Sie beruhen damit einerseits auf der Feststellung, dass eine vollständige Überwachung nicht möglich oder sinnvoll ist, gerade im Kontext freiheitlicher Gesellschaften, sowie letztlich andererseits auf verhaltenswissenschaftlichen Erkenntnissen, wie sie sich im Konzept des Bounded Opportunism ${ }^{902}$ widerspiegeln, welche auch die intrinsische Motivation zu wertekonformem Verhalten hervorheben.

An der Verankerung anhand einer Kultur der Integrität ist jedoch gerade vor dem Hintergrund unterschiedlicher Begriffsbestimmungen auch Kritik geübt worden. Diese Anmerkungen lassen sich, ähnlich zum geäußerten Relativismusvorwurf einer rein deskriptiven Ethik bzw. einer potentiellen „Kultur der Teufel“, kompakt so subsumieren, dass schließlich ebenfalls bspw. mafiöse Organisationen „Integrität“ aufweisen, also verlässlich, treu und nach bestimmten internen Werten verpflichtet handeln. ${ }^{903}$ Eine solche Auffassung karikiert allerdings zum einen die vielfach existierenden, bereits normativ geprägten Begriffsauffassungen ${ }^{904}$,

\footnotetext{
${ }^{900}$ So stellt auch KELLER in diesem Zusammenhang fest: „Integrität bedeutet jedoch mehr, denn es handelt sich dabei um eine Haltung.“ (Keller, T. (2019), S. 5).

${ }^{901}$ In diesem Sinne umfasst ein Integritätsmanagement auch Elemente bzw. Überlegungen des Complianceansatzes, geht jedoch deutlich darüber hinaus. Vgl. hierzu auch Keller, T. (2019), S. 5; Noll, B. (2013), S. 186; Paine, L. S. (1994), S. 113.

902 Vgl. hierzu nochmals Abschnitt 4.6.1.

${ }^{903}$ Vgl. Maak, T. / Ulrich, P. (2007), S. 10-11; Osterloh, M. / Weibel, A. (2006), S. 58-60. So stellen auch GRASSL und SCHMUTZ in Bezug auf ein rein deskriptives Begriffsverständnis fest, dass in diesem Falle ,Integrität zunächst nichts über die Qualität der persönlichen Werte aussagt. Sie ist insofern neutral, was die Art der Werte eines Menschen angeht. Auch ein Mafiaboss kann integer leben, wenn er seinen Werten und Überzeugungen folgt." (Grassl, J. / Schmutz, C. R. (2014), S. 44).

904 Vgl. hierzu auch Keller, T. (2019), S. 4-5, welche in diesem Sinne auch den Begriff der Loyalität anstelle der Integrität gebraucht.
} 
welche eben nicht nur als formales Prinzip einer konsistenten Normverfolgung auftreten, sondern vielmehr auf einem bestimmten inhaltlich (humanistisch) vorgeprägten Verständnisses dessen, was integer verfolgt wird, aufbauen. Darüber hinaus lassen sich die kritischen Anmerkungen hinsichtlich einer unterstellten Beliebigkeit des Begriffsinhalts der Integrität gerade vor dem Hintergrund des vorgestellten zweistufigen Legitimitätsansatzes deutlich entkräften bzw. die Frage, nach welchen Werten die Integrität zu bestimmen sei, nochmals konzeptionell präziser fassen. So kann, basierend auf dem elaborierten zweistufigen Legitimitätsansatz, festgestellt werden, dass Integrität auf eine verinnerlichte, mit legitimen Mitteln stattfindende Verfolgung legitimer unternehmerischer Ziele zur Erfüllung (realisierbarer) legitimer Stakeholderansprüche fokussiert. Legitimität ist damit erstens nicht beliebig oder lediglich anhand der aktuellen Wertvorstellungen der handelnden Personen bzw. der Organisation zu bestimmen, sondern vielmehr nach der gesellschaftlichen Moral. Darüber hinaus ist auch zweitens eine kritische Reflexion ${ }^{905}$ dieser vorherrschenden Moral an universellen Standards sowie ggf. fundierter normativer Konzepte möglich. Der Begriff der Integrität ist folglich erst recht vor diesem konzeptionellen Hintergrund nicht inhaltlich beliebig disponibel oder ethisch unterbestimmt. Eine Kultur, die in diesem Sinne ein Handeln mit Integrität, also eine verlässliche bzw. verinnerlichte (d. h. intrinsisch als bedeutsam empfundene) Beachtung legitimer Stakeholderansprüche verstärkt, kann hierdurch die Integration ethischer Maßnahmen und die Verwendung von Instrumenten wie dem Analytischen Framework klar unterstützen. In jedem Falle gilt bei der Implementation unternehmensethischer Maßnahmen auch die Relevanz der Unternehmenskultur zu beachten - oder: „,culture matters!“9906

\footnotetext{
905 Auch BESCHORNER betont die Relevanz einer „Ermöglichung reflexiver Freiräume“ (Beschorner, T. (2010), S. 120) im Rahmen von Integritätsansätzen.

906 Vgl. hierzu auch Sims, R. R. / Brinkmann, J. (2003), insbesondere mit Bezug auf den Untergang des Unternehmens Enron.
} 
Open Access Dieses Kapitel wird unter der Creative Commons Namensnennung 4.0 International Lizenz (http://creativecommons.org/licenses/by/4.0/deed.de) veröffentlicht, welche die Nutzung, Vervielfältigung, Bearbeitung, Verbreitung und Wiedergabe in jeglichem Medium und Format erlaubt, sofern Sie den/die ursprünglichen Autor(en) und die Quelle ordnungsgemäß nennen, einen Link zur Creative Commons Lizenz beifügen und angeben, ob Änderungen vorgenommen wurden.

Die in diesem Kapitel enthaltenen Bilder und sonstiges Drittmaterial unterliegen ebenfalls der genannten Creative Commons Lizenz, sofern sich aus der Abbildungslegende nichts anderes ergibt. Sofern das betreffende Material nicht unter der genannten Creative Commons Lizenz steht und die betreffende Handlung nicht nach gesetzlichen Vorschriften erlaubt ist, ist für die oben aufgeführten Weiterverwendungen des Materials die Einwilligung des jeweiligen Rechteinhabers einzuholen.

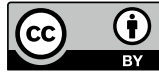

